\title{
The Impact of Symbiont Diversity on Cellular Integration and Function in the Cnidarian-Dinoflagellate Symbiosis
}

Amirhossein Gheitanchi Mashini

\author{
A thesis submitted to the Victoria University of Wellington \\ in fulfilment of the requirements for the degree of \\ Doctor of Philosophy
}

Victoria University of Wellington

2022 
There was a door to which I found no key There was a veil past which I could not see Some little talk awhile of me and thee

There seemed--and then no more of thee and me.

Omar Khayyam

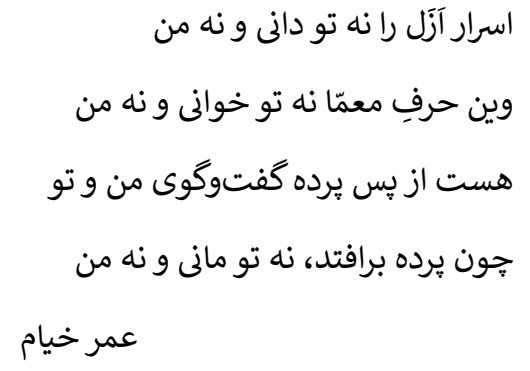




\begin{abstract}
Coral reef ecosystems are one the most important tropical ecosystems, providing a wide variety of ecological services. Reef-building corals form a symbiosis with phototrophic dinoflagellates of the family Symbiodiniaceae, however elevated temperatures disrupt this relationship in a process known as coral bleaching and worldwide bleaching events are increasing in frequency and intensity due to climate change. There is considerable interest in whether corals might adapt to warming conditions by changing their symbionts to a more thermally-tolerant type. The potential to respond is related to capacity for cellular integration between the partners, which underlies the specificity of the host-symbiont relationship. The aim of this thesis was to elucidate the cellular processes associated with hosting native $v s$. non-native symbionts in the model symbiotic cnidarian Exaiptasia pallida (commonly referred to as 'Aiptasia'), to assess the potential for establishing a novel partnership. A multidisciplinary approach, including proteomics and quantitative immunocytochemistry, was used, with a particular focus on processes involved in inter-partner nutritional exchange.
\end{abstract}

In Chapter 2, a new method for characterising the proteome of Symbiodiniaceae is described. This method was used to compare the molecular and metabolic pathways underlying a successful symbiosis. Specifically, the proteome of Breviolum minutum, the native symbiont of Aiptasia, was compared when in culture (i.e., free-living) vs. in symbiosis, under a range of nutritional regimes (i.e., N-deplete or N-enriched in culture; starved or well-fed anemones in symbiosis). These various treatments induced distinct proteomes in B. minutum, especially related to immunosuppression to avoid host cell phagosome maturation, metabolic integration, and increased oxidative stress in the symbiotic state. Moreover, the different nutritional regimes impacted the $B$. minutum proteome, with evidence for increased efficiency nutrient uptake and assimilation under more nutrient-limited conditions.

Chapter 3 further characterised the proteome of $B$. minutum during the process of host colonization, and then compared this to the proteome of a non-native symbiont, the putatively opportunistic but thermally tolerant Durusdinium trenchii, when in symbiosis with Aiptasia. During host colonization over a fourteen-week period, the proteome of B. minutum showed changes related to photosynthesis and chloroplast maintenance as the symbiont density 
increased, until it eventually reached its peak. Conversely, the proteome of the non-native $D$. trenchii exhibited a lower abundance of photosynthetic proteins in the fully-established symbiosis, but an upregulation of parasite-like immunosuppression mechanisms, consistent with the view of this species of Symbiodiniaceae being opportunistic.

Lastly, in Chapter 4, host nutrient transporters were localized and quantified in Aiptasia, colonized with either the native B. minutum or the non-native D. trenchii and Symbiodinium microadriaticum. Specific immunofluorescent antibodies were designed for four different host transporters of interest: ammonium transporter 1 (AMT1); V-type proton ATPase (VHA); facilitated glucose transporter member 8 (GLUT8); and aquaporin-3 (AQP3, a sugar transporter). Tissue sections were then analysed by confocal microscopy. Hosts harbouring the non-native symbionts showed different transporter localization patterns to those harbouring the native $B$. minutum, suggesting a lesser degree of host-symbiont integration and disrupted nutritional flux with the non-native symbionts, with $S$. microadriaticum-colonized anemones being particularly distinct.

This thesis therefore adds further weight to the view that the cellular integration necessary to establish a functional symbiosis, with efficient inter-partner nutritional exchange, is not necessarily replicated with non-native symbiont species, which in some cases may persist even when symbiosis function is compromised. Ultimately, this will likely reduce the likelihood that corals might adapt to climate change by changing their symbiont population. On a more positive note, however, this thesis provides new insights into the fundamental cellular mechanisms that underlie a successful cnidarian-dinoflagellate symbiosis and contribute to observable patterns of host-symbiont specificity. Such information is essential if we are to develop the tools necessary for saving the world's coral reefs, for example through gene-editing and the engineering of more thermally tolerant coral-dinoflagellate partnerships. 


\section{Acknowledgments}

First and foremost I am extremely grateful to my supervisors, Prof. Simon Davy and Dr. Clint Oakley for their invaluable advice, continuous support, and patience during my $\mathrm{PhD}$ study. Their immense knowledge and plentiful experience have encouraged me in all the time of my academic research and daily life. I would also like to thank Professor Virginia Weis and Professor Arthur Grossman for their advice and support on my study. I would like to thank my friends, Hayley, Naji and Mohammad, and more importantly My Mum. Without their tremendous understanding, support, and encouragement in the past few years, it would be impossible for me to complete my study. 


\section{Table of contents}

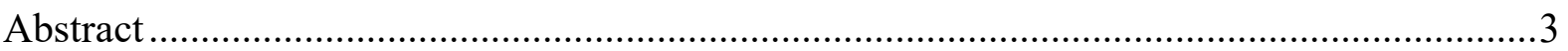

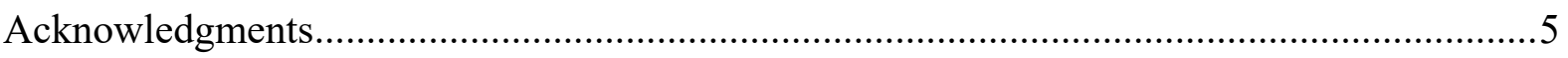

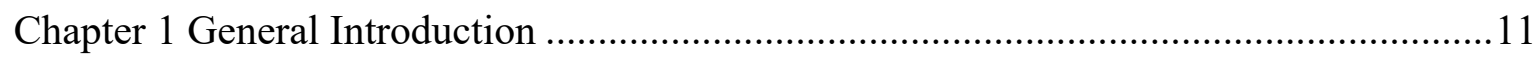

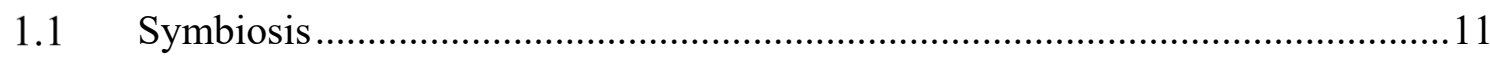

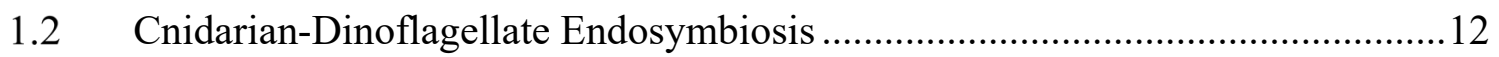

1.2.1 Architecture of Cnidarian-Dinoflagellate Endosymbiosis ..........................12

1.2.2 Acquisition, Establishment and Regulation of Symbionts ..........................14

1.3 Metabolic Exchange and Nutrient Trafficking ............................................... 16

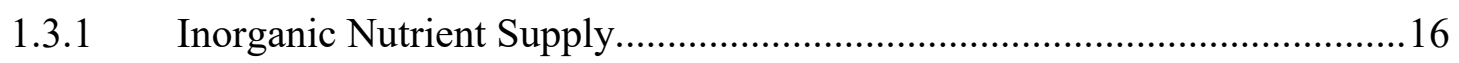

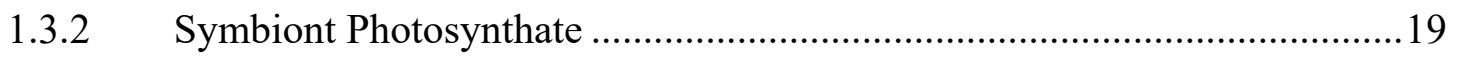

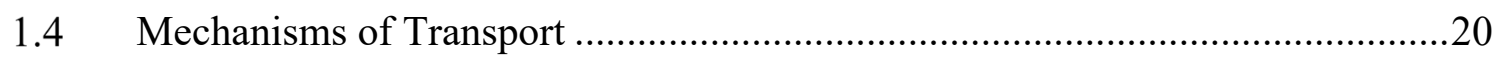

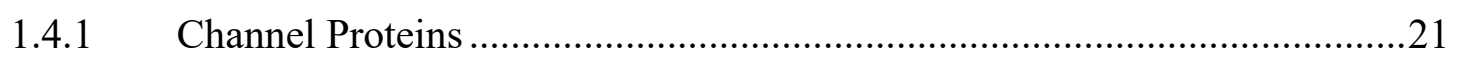

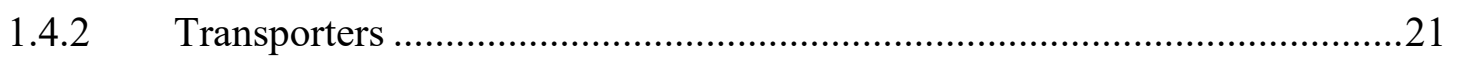

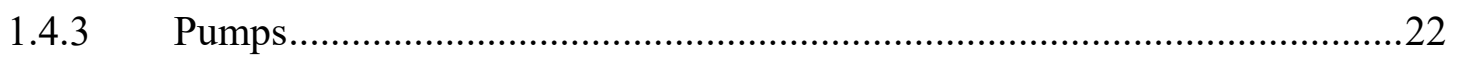

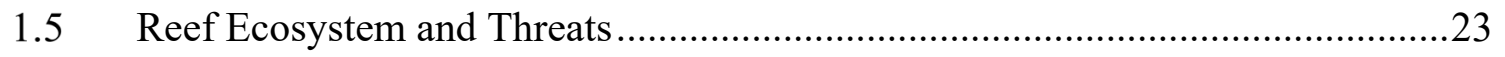

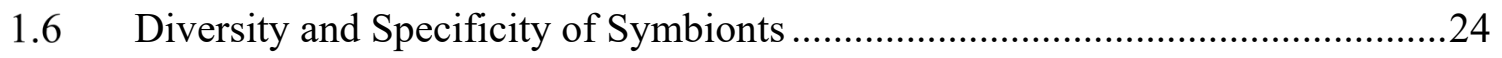

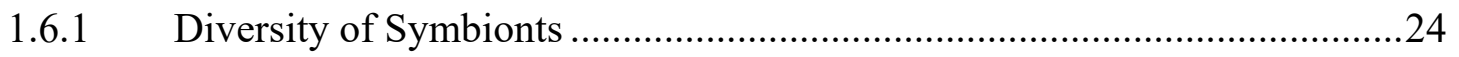

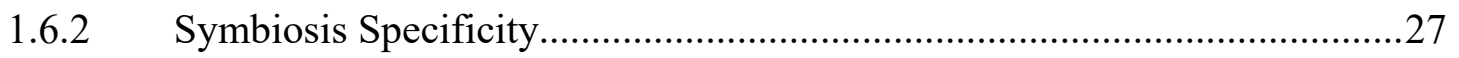

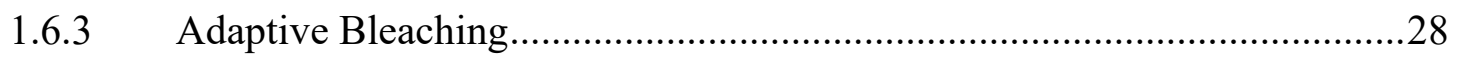

1.7 Cnidarian-Dinoflagellate Symbiosis in "Omics” Era .........................................29

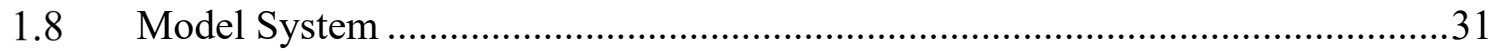

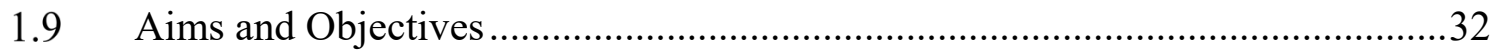

Chapter 2 The influence of symbiosis on the proteome of the symbiotic dinoflagellate

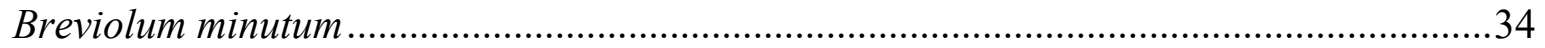

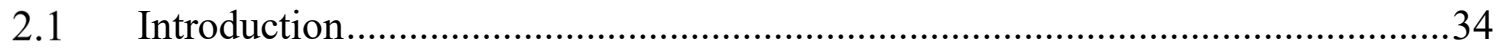

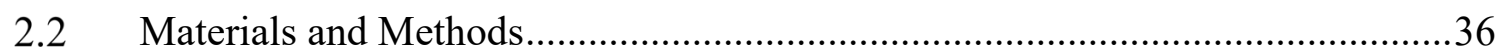

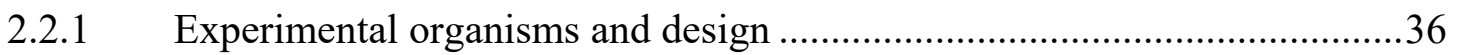

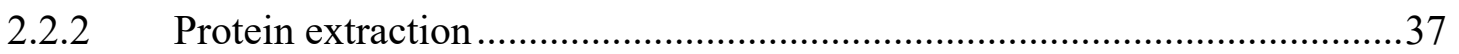

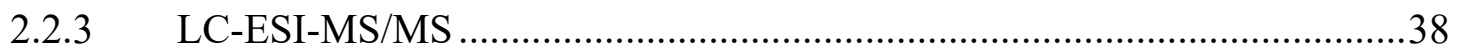

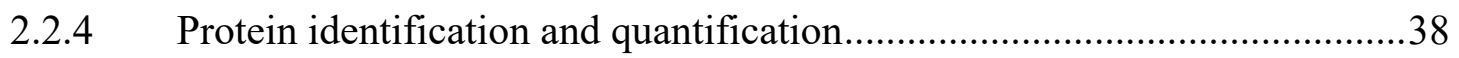

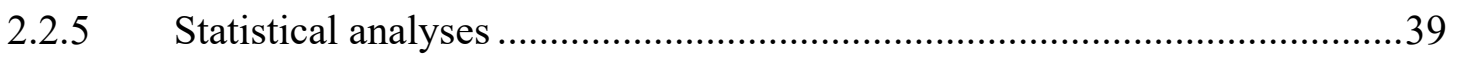

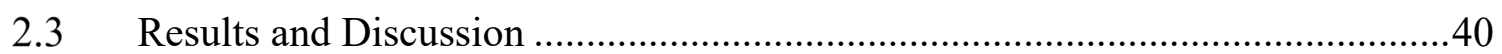


2.3.1 Endosymbiont density and photobiology.

2.3.2 Proteins affected by symbiotic state and nutritional regime.......................41

2.3.3 Proteomic shifts associated with symbiotic state .......................................42

2.3.4 Proteomic shifts associated with nutritional state .....................................49

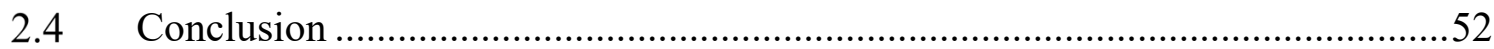

Chapter 3 The influence of symbiosis on the proteome of a native and non-native symbiont

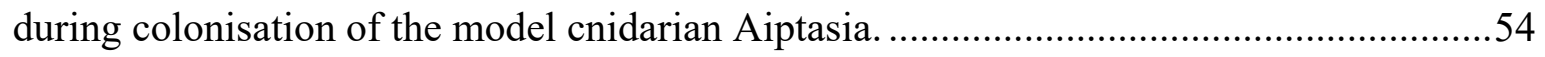

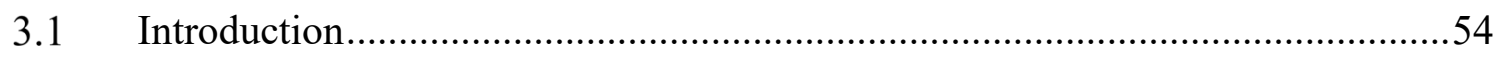

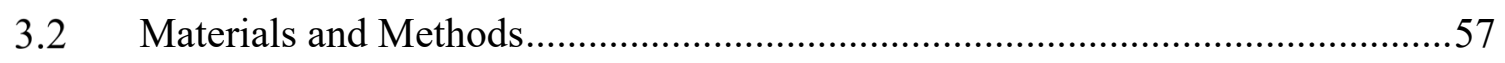

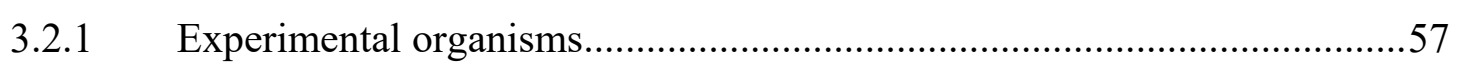

3.2.2 Inoculation of Aiptasia with symbiotic algae ...........................................57

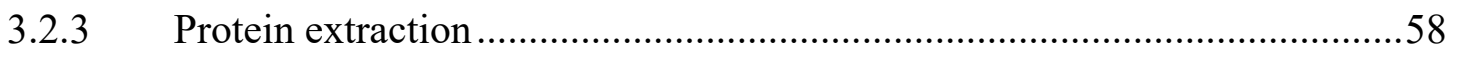

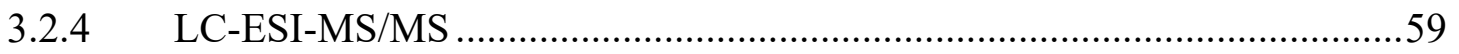

3.2.5 Protein identification and quantification...................................................59

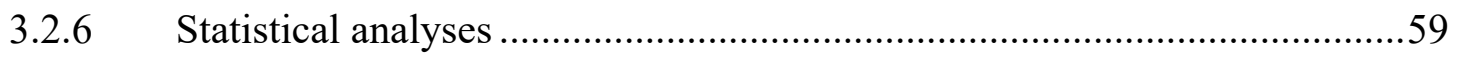

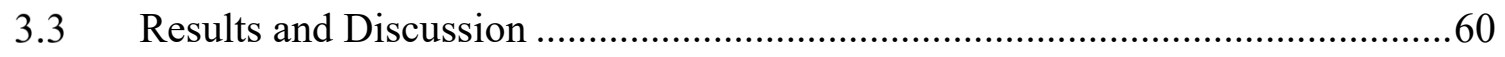

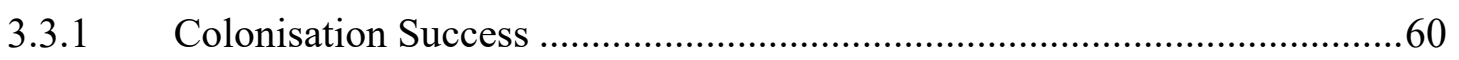

3.3.2 Differentially expressed proteins in B. minutum vs. D. trenchii ..................61

3.3.3 B. minutum proteome changes over the course of host colonization............62

3.3.4 Comparative responses of $B$. minutum vs. D. trenchii .................................64

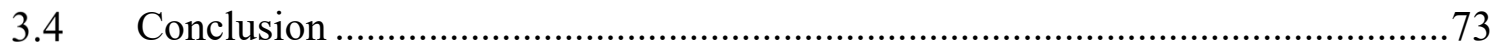

Chapter 4 Immunolocalization of metabolite transporter proteins in a model cnidarian-

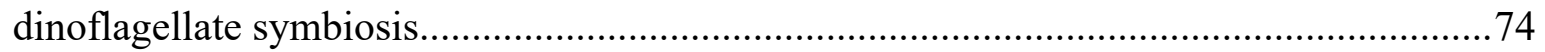

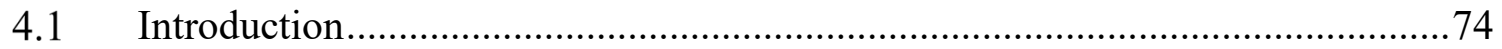

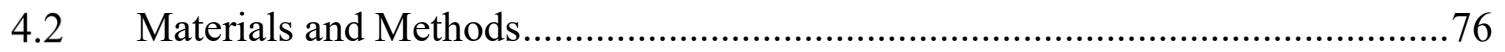

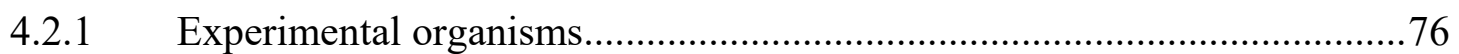

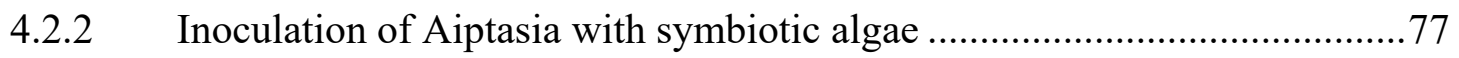

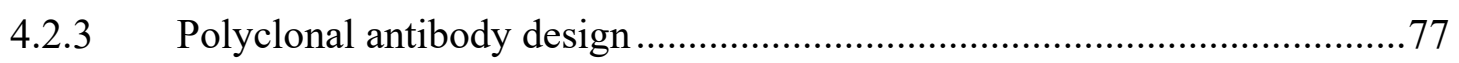

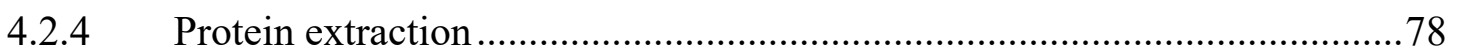

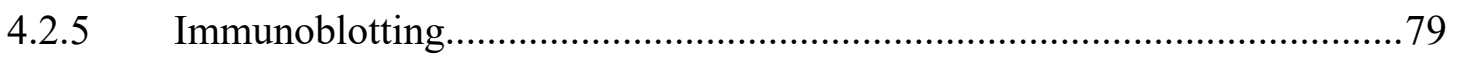

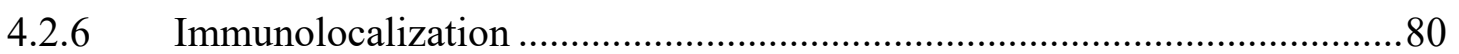

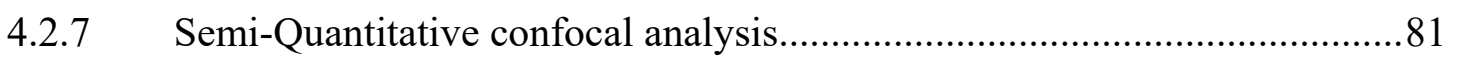

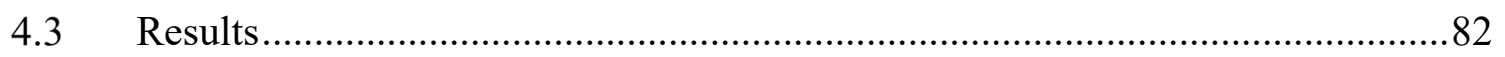

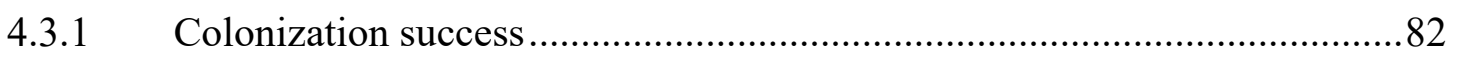

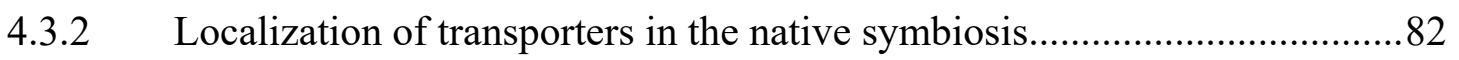


4.3.3 Effects of symbiont identity on transporter localization...........................83

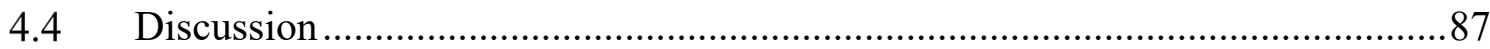

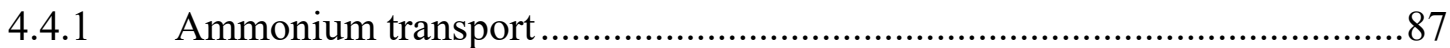

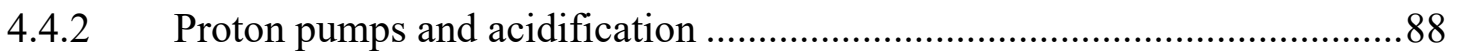

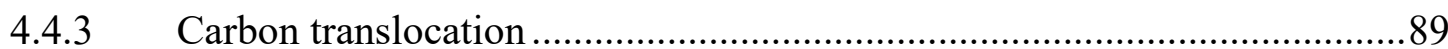

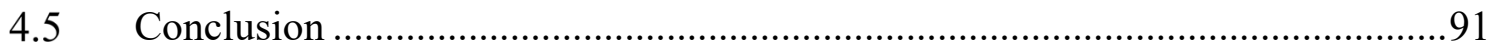

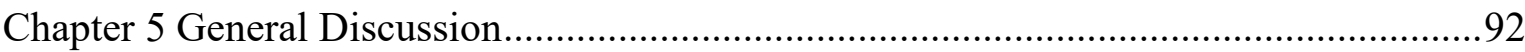

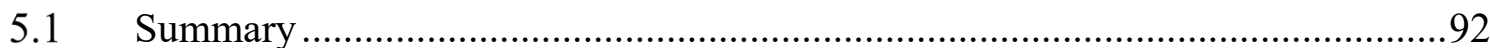

5.2 Partner integration and symbiosis function......................................................93

5.2.1 What is the potential for the evolution of novel cnidarian-dinoflagellate symbioses?

5.2.2 Does the degree of cellular integration between the symbiotic partners reflect the position of the symbiosis on the mutualism - parasitism continuum? .......97

5.2.3 What are the ecological implications for the survival of coral reefs in the face of climate change, and the development of strategies for increasing reef resilience?

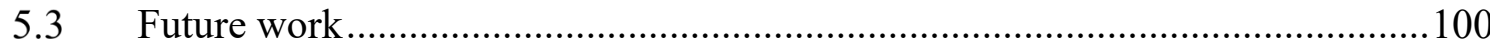

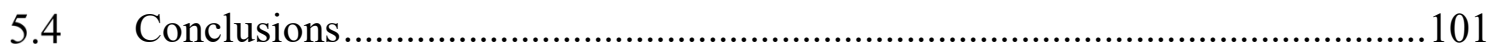

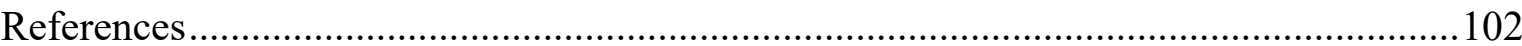

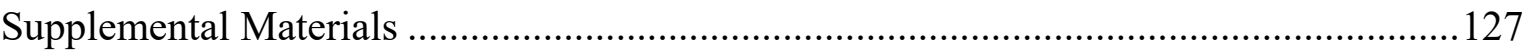




\section{List of Figures}

Figure 1.1. The symbiotic dinoflagellate Breviolum minutum in Exaiptasia pallida ..............13

Figure 1.2. The establishment and persistence in cnidarian- algal symbiosis ........................16

Figure 1.3. Metabolic interactions in the cnidarian-dinoflagellate symbiosis .........................19

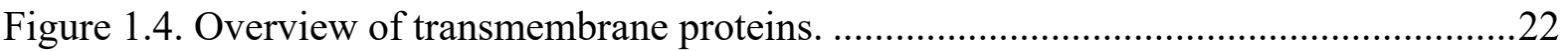

Figure 1.5. Phylogenetic relationships between Symbiodiniaceae .........................................26

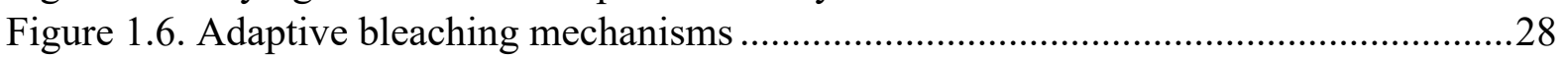

Figure 1.7. Antibodies and their application in immunocytochemistry.................................

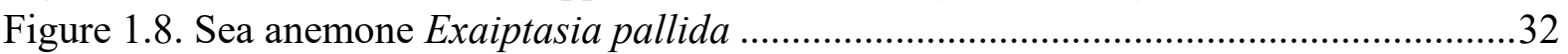

Figure 2.1. Physiological effects of nutritional regimes in $B$. minutum .................................40

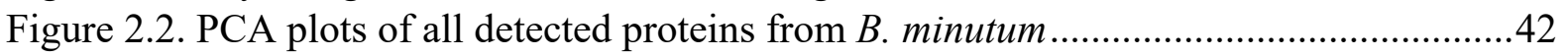

Figure 3.1. Population density of B. minutum and Durusdinium trenchii in Aiptasia............61

Figure 3.2. PCA plots of detected symbiont proteins during colonization of Aiptasia...........62

Figure 4.1. Colonization densities of different symbiont species in Aiptasia. .......................82

Figure 4.2. Immunofluorescence localization of nutrient transporters in Aiptasia..................84

Figure 4.3. Signal intensities of quantified transporters in Aiptasia tentacles ........................85

Figure 4.4. Intracellular localization of transporters on Aiptasia cells ..................................86

Figure 5.1. Conceptual diagram of the cellular processes and symbiont compatibility .........97 


\section{List of Tables}

Table 2-1. Summarized list of proteins from B. minutum (in hospite vs. ex hospite)..............44 Table 2-2. Summarized list of proteins from B. minutum (high nutrient $v s$. low nutrient)......51

Table 3-1. Summarized list of proteins from B. minutum during colonization of Aiptasia.....66

Table 3-2. Summarized list of proteins of comparision of $B$. minutum and D. trenchii ..........71 


\section{Chapter 1 \\ General Introduction}

\subsection{Symbiosis}

Symbiosis, meaning living together, is referred to as an interaction of two or more kinds of organisms in often elaborate and more-or-less permanent close physical relationships (Paracer and Ahmadjian, 1986). Symbiotic relationships can be different types such as: (1) commensalism, in which one species benefits and the other neither benefits nor is harmed; (2) mutualism, in which both participating species benefit; and (3) parasitism, in which one species benefits but the other is harmed. Parasitism can also be viewed as a form of predation, although the organism that is preyed on does not necessarily die (Mason et al., 2017). Symbiotic relationships can be also classified based on the location and dependence on the symbiosis, as a symbiont partner (i.e. the smaller partner) may reside outside of a host cell (ectosymbiosis) or inside a host cell (endosymbiosis). In the case of dependence, partners can be totally dependent on the relationship for survival (obligatory) or be capable of surviving in a freeliving state (facultative) (Paracer and Ahmadjian, 1986). Nevertheless, all of these relationships have potential for coevolution (a situation under which organisms evolve based on interspecies interactions) between the organisms involved, and in many instances, interspecies coevolution has resulted in fascinating complexes (Mason et al., 2017). For instance, the mitochondria and the plastids of eukaryotic cells arose by one cell engulfing another cell, forming an endosymbiotic relationship. Subsequently, the ingested cell lost its autonomy and some of its functions. In addition, many of the ingested cell's genes were transferred to the host's DNA. Thus, mitochondria and plastids in modern eukaryotic cells are the remnants of these symbionts, retaining some specialized functions that benefit their host cells (Margulis, 2013; Sadava et al., 2011).

Symbiotic associations are common in nature, ranging from integrated molecular symbiotic interactions of viruses with their hosts, to parasitic plants lacking chlorophyll, to mutualistic associations between sharks and remora (Camisão and Pedroso, 2013; Kloc, 2020; Norman et al., 2021). Regardless of the type of symbiosis, most organisms that are involved acquire new phenotypic capability and therefore possibility for conferring selective advantages and potentially exploiting new (or further securing existing) environmental niches (Moran, 2007). 
For example, the symbiotic relationship between sulphide-oxidising bacteria and giant tube worms has provided selective advantages for exploiting deep-sea habitats and formed ecosystems completely independent of sunlight around deep-sea hydrothermal vents (Childress et al., 1987; Dick, 2019). The cnidarian-dinoflagellate association is another symbiotic interaction which plays a key ecological role, in this case the functioning of coral reef ecosystems (Muscatine and Porter, 1977; van Oppen and Medina, 2020). This symbiotic association forms one of the most biologically diverse and productive ecosystems on the planet (Hoegh-Guldberg et al., 2007), which provides human communities with benefits such as food, income, recreation, coastal protection and cultural settings (Hoegh-Guldberg et al., 2017; Kleypas et al., 2021).

\subsection{Cnidarian-Dinoflagellate Endosymbiosis}

Endosymbiotic relationships with photosynthetic algae are a common phenomenon in members of the phylum Cnidaria (Hofmann and Kremer, 1981). For instance, the symbiotic relationship between the scyphozoan Cassiopeia xamachana and dinoflagellates of the genus Symbiodinium, or the freshwater hydroid Hydra viridis and green algae of the genus Chlorella (Trench, 1993). The most ecologically significant symbiosis in the phylum is found on coral reefs. Here, the symbiosis with members of the family Symbiodiniaceae provides the coral host with photosynthetic products such as glucose (Hillyer et al., 2017b), which support coral metabolism, reproduction and survival. In return, the host is responsible for supplying inorganic carbon, nitrogen and phosphorus which are required for photosynthesis and growth in tropical oligotrophic habitats (Davy et al., 2012). A shortage of energy acquired from feeding on exogenous sources makes some corals primarily dependent on their symbionts (Davies, 1984; Johannes et al., 1970). Moreover, symbionts enhance the rate of coral skeletogenesis (Comeau et al., 2017), which in the long-term results in being maintained in a well-lit environment while having more protection from grazers (Davy et al., 2012).

\subsubsection{Architecture of Cnidarian-Dinoflagellate Endosymbiosis}

Dinoflagellates are found in the gastrodermal cells of the cnidarian host (Muscatine et al., 1998), where they are surrounded by a series of membranes of algal origin and an outermost host-derived membrane; together these form the symbiosome membrane complex (Fig. 1.1) 
(Kazandjian et al., 2008; Wakefield and Kempf, 2001). Multiple underlying membranes of algal origin are hypothesised to originate from repeated delayed ecdysis by symbionts, during which the plasma membrane and thecal vesicles are shed and new sets form beneath (Wakefield et al., 2000). The outermost host-derived symbiosome membrane is a retained early phagosome, where maturation has been arrested via preventing ApRAB7 by the symbiont (Chen et al., 2004, 2003). Indeed, all nutrients and metabolites must pass across this single bilayer membrane when being transferred between host and symbiont, and vice versa (Kazandjian et al., 2008).
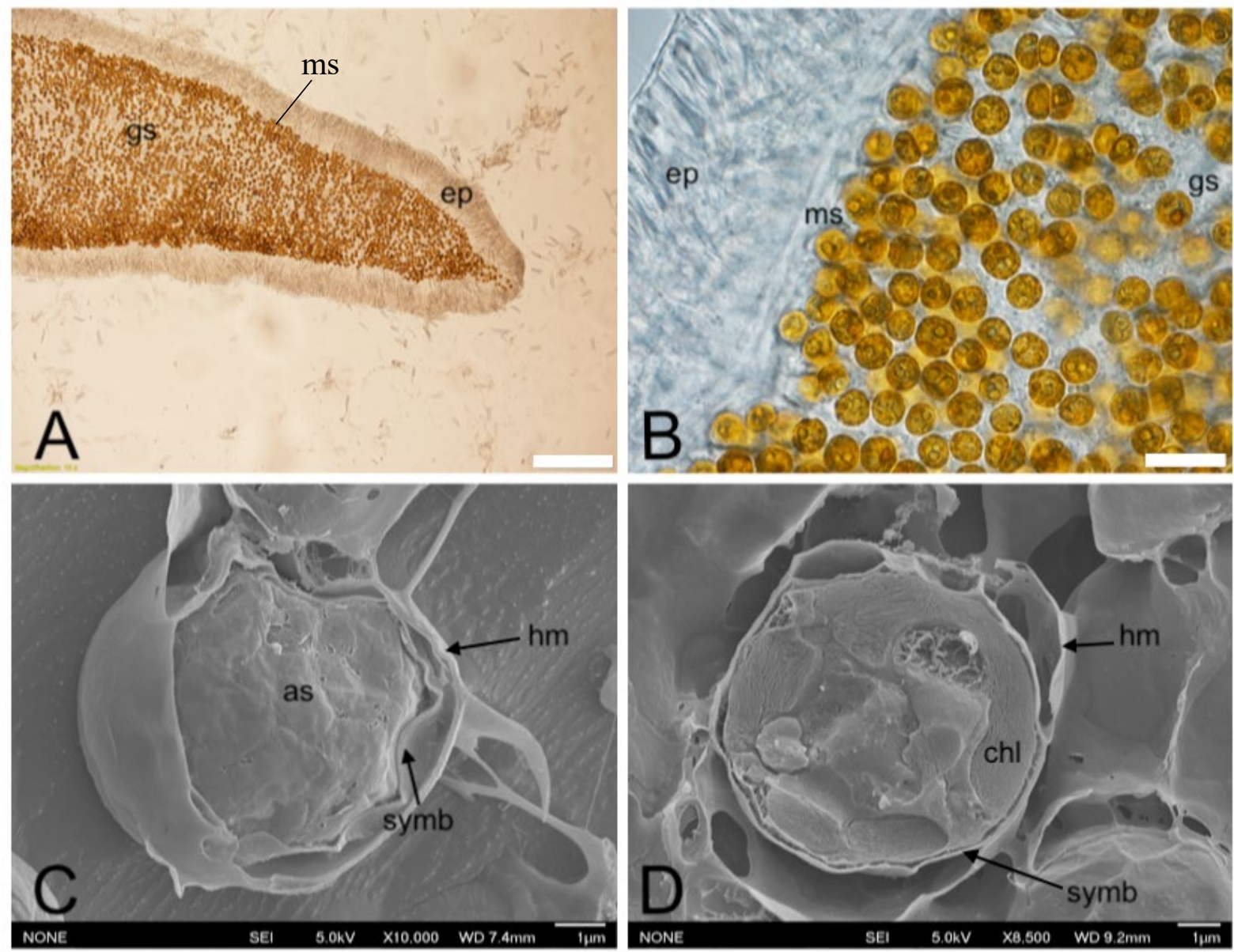

Figure 1.1. The symbiotic dinoflagellate Breviolum minutum in the sea anemone 'Aiptasia' (Exaiptasia pallida). (A) Tentacle showing gastrodermis and epidermis; scale $100 \mu \mathrm{m}$. ep = epidermis, gs = gastrodermis. (B) Symbionts (round yellow-brown cells) in gastrodermis layer; Scale $10 \mu \mathrm{m}$. ep = epidermis, gs = gastrodermis, ms = mesogloea. (C) and (D) Scanning Electron Microscopy (SEM) freeze-fractured Aiptasia tentacle showing host and algae inside it; scale $1 \mu \mathrm{m}$. as = algal symbiont, symb $=$ symbiosome membrane, $\mathrm{hm}=$ host membrane, $\mathrm{chl}=$ chloroplast.

In addition to Symbiodiniaceae, corals may host bacteria, archaea, fungi, viruses and protists. All of these together (including the host) are referred to as the 'holobiont' (Rohwer et al., 2002; Rosenberg et al., 2007). Interactions of holobiont members are likely to aid host function and 
survival (Bernasconi et al., 2019; Matthews et al., 2020; Rosenberg et al., 2007).

\subsubsection{Acquisition, Establishment and Regulation of Symbionts}

Most cnidarians obtain their algal partner by horizontal transmission, which involves acquisition of the symbiont from the surrounding environment after spawning. However, some cnidarians inherit their symbionts from parents, which is known as vertical (or maternal) transmission. The transmission mode of symbionts is correlated with host reproductive traits (Stat et al., 2006). The majority of coral species are broadcast spawners (releasing gametes into the surrounding environment) and therefore, acquire algal symbionts from the environment during planular larval or juvenile colony stages (Baird et al., 2009; Little et al., 2004; Stat et $a l ., 2006)$. In vertical transmission, offspring are provided with obligate symbionts that already 'fit' the species and current ecological environment. In spite of this, if a coral planula settles in a different environment, it can be disadvantaged, as the symbiont might not perform optimally (Byler et al., 2013; Weis et al., 2001). Although vertical transmission is the primary mode for obtaining symbionts in brooder species (retaining offspring in the parental colony), some acquire novel symbionts by horizontal transmission, because it provides an opportunity for taking up ecologically suitable partners, as host survival depends on an unpredictable symbiont source (Byler et al., 2013).

Both partners first need to recognize each other in advance of symbiosis establishment. Hostsymbiont recognition processes are described as 'winnowing', that includes a cascade of events, all of which are necessary and related to each other (Cooper, 2007; Nyholm and McFallNgai, 2004). Innate immune repertoires in invertebrates are sophisticated, complex and have important roles in the establishment of symbiosis, and it has been suggested that host recognition mechanisms of Symbiodiniaceae are similar to recognition of pathogenic organisms in eukaryotes (Kirk and Weis, 2016; Weis, 2008). Symbiont recognition and uptake are performed by pattern recognition receptors (PRRs) that recognise and further bind to conserved components of algal surface molecules such as carbohydrates, proteins and lipids (Kilpatrick, 2002). For example, glycoproteins covering the algal surface are reported to be involved in lectin/glycan recognition interactions with host lectin receptors (Lin et al., 2000; Logan et al., 2010; Neubauer et al., 2016; Wood-Charlson and Weis, 2009). In addition, glycoproteins working as interspecies signals are secreted from symbionts both in and ex 
hospite (Markell and Wood-Charlson, 2010). Nonetheless, a recent study on glycan profiles of different symbionts only reported subtle differences between colonising and non-colonising symbionts, Breviolum minutum and Symbiodinium pilosum, respectively (Parkinson et al., 2018). In this latter study, glycan masking of B. minutum with two different lectins during colonisation of Aiptasia detected no significant difference in symbiont cell densities after reinfection, which further suggests that lectin/glycan interactions are more important for postphagocytic persistence of specific symbionts than they are for initial uptake. In addition, Niemann-Pick type C gene family (e.g. NPC2) and Sym32 proteins have been hypothesised to participate in the formation of cnidarian-dinoflagellate symbioses (Dani et al., 2017, 2014; Reynolds et al., 2000; Schwarz and Weis, 2003). In the next step, symbionts in the vicinity of the host gastrodermis are ingested via phagocytosis, when the host determines whether to accept or reject the new symbiont (Davy et al., 2012). Adult hosts can be selective, preferring more specific symbiont partners, while new recruits can be fairly unspecific (Weis et al., 2001; Little et al., 2004; Thornhill et al., 2006; Gabay et al., 2019, 2018). Recent research on the sea anemone Exaiptasia pallida (commonly referred to as 'Aiptasia') has shown that specificity is also dependent on symbiont cell size, as larger Symbiodiniaceae are less likely to form symbioses with Aiptasia; a similar phenomenon may also contribute to the host-symbiont specificity seen in corals (Biquand et al., 2017). A variety of mechanisms for rejection have been proposed, such as in situ digestion, exocytosis and host cell apoptosis (programmed cell death) (Weis, 2008). Interestingly, all dead symbionts that get phagocytosed are digested rapidly (Fitt and Trench, 1983), while algae that have been accepted will be kept in a haploid vegetative stage inside the host gastrodermis (Davy et al., 1997; Davy and Turner, 2003; Fitt and Trench, 1983). Certainly, further research is needed to fully understand the mechanisms involved in the establishment of the cnidarian-dinoflagellate symbiosis (Fig. 1.2).

A cnidarian host must regulate the population of its symbionts, otherwise symbionts may either reduce in number and thus not be productive enough, or overgrow the host (Neckelmann and Muscatine, 1983; Taylor et al., 1989). Cnidarian hosts have different mechanisms to ensure that optimal populations of symbionts are maintained in hospite, such as limiting nitrogen and phosphorus, which results in arrested cell growth and division of symbionts (Ezzat et al., 2016; Miller and Yellowlees, 1989; Muscatine and Pool, 1979; Smith and Muscatine, 1999). Furthermore, excessive algae are continuously removed through apoptosis, digestion of 
symbionts (autophagy), or expulsion of symbionts into the environment (exocytosis mechanisms) (Paxton et al., 2013; Weis, 2008).

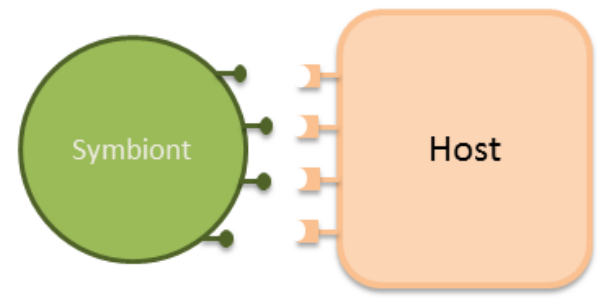

1. Initial Contact

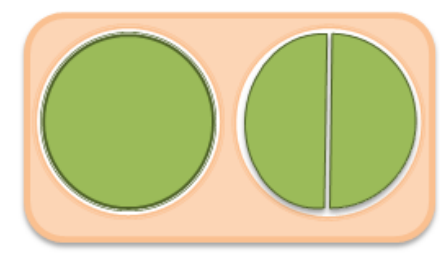

4. Proliferation

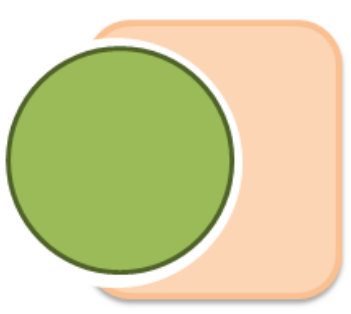

2. Phagocytosis

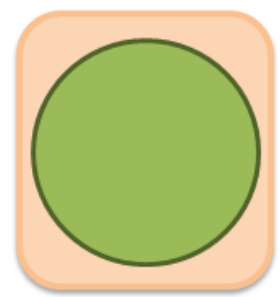

5. Dynamic stability

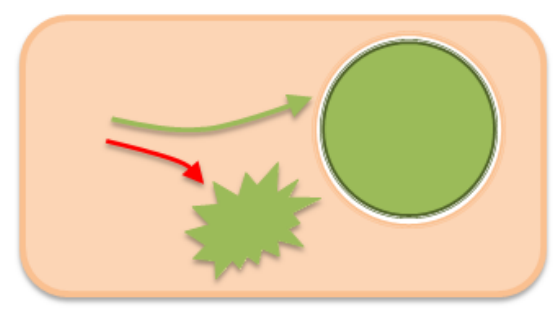

3. Sorting

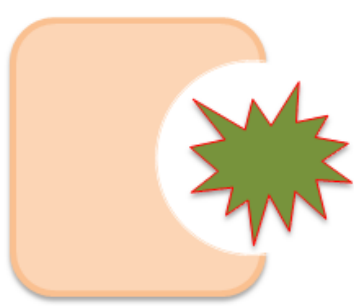

6. Dysfunction

Figure 1.2. The six phases of symbiosis establishment and persistence in cnidarian- algal symbiosis (based on Davy et al., 2012). 1. Initial surface contact between the algal symbiont and cnidarian host cell which involves surface glycoproteins; 2. Symbiont phagocytosis by the host cell; 3 . Sorting of the symbionts, resulting in either rejection (red arrow) or acceptance (green arrow); 4. Symbiont cell division within the host tissues; 5. Dynamic stability, when the symbiont population is regulated and functions properly; and 6, Symbiosis dysfunction and expulsion of the symbionts.

\subsection{Metabolic Exchange and Nutrient Trafficking}

As coral reefs are normally located in nutrient-depleted waters, metabolic exchange is key to a successful coral-dinoflagellate endosymbiosis (Davy et al., 2012; Matthews et al., 2020). This association involves bi-directional nutrient flow between partners: the dinoflagellate symbionts have a vital role in host nourishment, growth and calcification via the translocation of photosynthetic products, while in return the host provides its endosymbionts with sufficient nutrient pools for photosynthesis and respiration (Muscatine and Porter, 1977; Yellowlees et al., 2008).

\subsubsection{Inorganic Nutrient Supply}

The cnidarian host supplies the symbionts with inorganic compounds such as dissolved inorganic carbon (DIC), dissolved inorganic nitrogen (DIN) and phosphorus, plus various 
organic compounds (Davy et al., 2012). Of these, DIC is especially important as carbon compounds are central to photosynthesis. DIC can be taken up as $\mathrm{CO}_{2}$ resulting from symbiont and host respiration (Harland and Davies, 1995; Rädecker et al., 2017a) or skeletogenesis in the case of calcifying anthozoans (Comeau et al., 2017; Ware et al., 1992), or it can be taken up from the surrounding seawater (Allemand et al., 1998; Furla et al., 2000b). DIC in ambient seawater is mostly present as bicarbonate $\left[\mathrm{HCO}_{3}{ }^{-}\right]$and carbon dioxide $\left[\mathrm{CO}_{2}\right]$, however only $\mathrm{CO}_{2}$ can freely pass through cell membranes (Fig. 1.3). In contrast, bicarbonate is actively translocated via carbon concentrating mechanisms (CCMs) (Allemand et al., 1998). Host carbonic anhydrase (CA), which catalyses the conversion of bicarbonate to $\mathrm{CO}_{2}$ and vice versa, is integral to $\mathrm{CCMs}$ as it provides $\mathrm{CO}_{2}$ that is used for both photosynthesis and calcification (Furla et al., 2000b). A recent study showed that a vacuolar-type proton pump (V-type $\mathrm{H}^{+}$ ATPase) on the symbiosome membrane is also part of the CCMs. The cnidarian host acidifies the symbiosome vacuole in order to promote photosynthesis by concentrating $\mathrm{CO}_{2}$ (Barott et al., 2015b).

Nitrogen and phosphorus are also transferred between the partners in the cnidariandinoflagellate symbiosis (Ferrier-Pagès et al., 2018; Pernice et al., 2012; Wang and Douglas, 1998), and are important for a functional symbiosis (Fig. 1.3). However, nutrient-depleted waters surrounding coral reefs have relatively low concentrations of both $\mathrm{N}$ and $\mathrm{P}(<1 \mu \mathrm{M})$ (Miller and Yellowlees, 1989; Smith and Muscatine, 1999).

Nitrogen fluxes are one the most studied and significant nutritional cycles between the partners (Davy et al., 2012). Nitrogen in the ambient seawater is predominantly available as inorganic ammonium $\left(\mathrm{NH}_{4}^{+}\right)$and nitrate $\left(\mathrm{NO}_{3}{ }^{-}\right)$ions, or dissolved organic nitrogen (DON) in the form of free amino acids (FAA) and urea (D'Elia et al., 1983; Grover et al., 2008, 2003; Yellowlees et al., 2008). Nitrogen is also available as particulate organic nitrogen (PON) such as plankton (Davy et al., 2006; Ezzat et al., 2017). Although both symbiotic partners can assimilate ammonium, the dinoflagellate symbionts are major players in this (Grover et al., 2002). The two partners have different mechanisms for nitrogen assimilation: the cnidarian host primarily employs the NADP-glutamate dehydrogenase (NADP-GDH) pathway while the algal partner prefers the glutamine synthetase/glutamine 2-oxoglutarate amido transferase (GS/GOGAT) pathway. Nevertheless, both pathways result in glutamate as the final product, which is a 
precursor for other amino acids (Miller and Yellowlees, 1989; Roberts et al., 2001; Summons and Boag, 1986; Wilkerson and Muscatine, 1984). Symbionts can also obtain ammonium from host catabolism of nitrogenous compounds (Cates and McLaughlin, 1976; Ezzat et al., 2017; Pernice et al., 2012; Rädecker et al., 2017b; Rahav et al., 1989). Nitrate ions must be first converted to ammonium before assimilation in a process which requires nitrate and nitrite reductase enzymes (Tanaka et al., 2006), and as cnidarians lack these enzymes (Crossland and Barnes, 1977), assimilation exclusively takes place in the dinoflagellate partner (Kopp et al., 2013; Leggat et al., 2007). Nitrogen compounds obtained directly by the algae or translocated by the host are either metabolised or stored by the symbionts, and may be translocated to the cnidarian host in the form of amino acids (nitrogen recycling) (Kopp et al., 2013; Reynaud et al., 2009; Wang and Douglas, 1999, 1998). Host metabolic ammonium production can be also reduced in response to the provision of organic carbon by the symbionts (nitrogen conservation) (Wang and Douglas, 1998).

Studies investigating phosphorus fluxes in the cnidarian-dinoflagellate symbiosis are scant relative to those focused on nitrogen (Davy et al., 2012). Phosphorus is available for the cnidarian-dinoflagellate holobiont in particulate, and dissolved organic and inorganic forms (Ferrier-Pagès et al., 2016). Dissolved inorganic phosphorus (DIP), mainly phosphate [PO4$\left.{ }^{3-}\right]$, is the most accessible form (Bjorkman and Karl, 1994). However, phosphate assimilation is a complex process that involves the host, symbionts, and their environmental conditions, and therefore remains difficult to model (Ferrier-Pagès et al., 2016). Phosphate is charged naturally at physiological and seawater $\mathrm{pH}$, and therefore it needs to be actively transported from ambient seawater against a concentration gradient into the host-cell cytoplasm and then the symbionts (Ferrier-Pagès et al., 2016; Godinot et al., 2009; Jackson and Yellowlees, 1990). Particulate phosphate can be also acquired by heterotrophic feeding, which needs to be broken down to DIP before assimilation (Ferrier-Pagès et al., 2016).

In addition to these nutrients, fatty acids, lipids. the amino acid glycine and sulphur containing amino acids are translocated to the algal partners (Fig. 1.3) (Cook, 1971; Hillyer, 2016; Imbs et al., 2014; Von Holt, 1968; Wang and Douglas, 1999). These various nutritional fluxes are extremely important for the algal partner's growth, division and photosynthesis, and also for host metabolism. Moreover, DIN and DIP concentrations may be important factors for 
maintaining symbiont cell density during exposure to thermal stress (Ezzat et al., 2016). Therefore, investigating nutrient fluxes in different functional symbioses can broaden our knowledge of the associated nutritional pathways and their physiological benefits under both normal and stressful conditions.

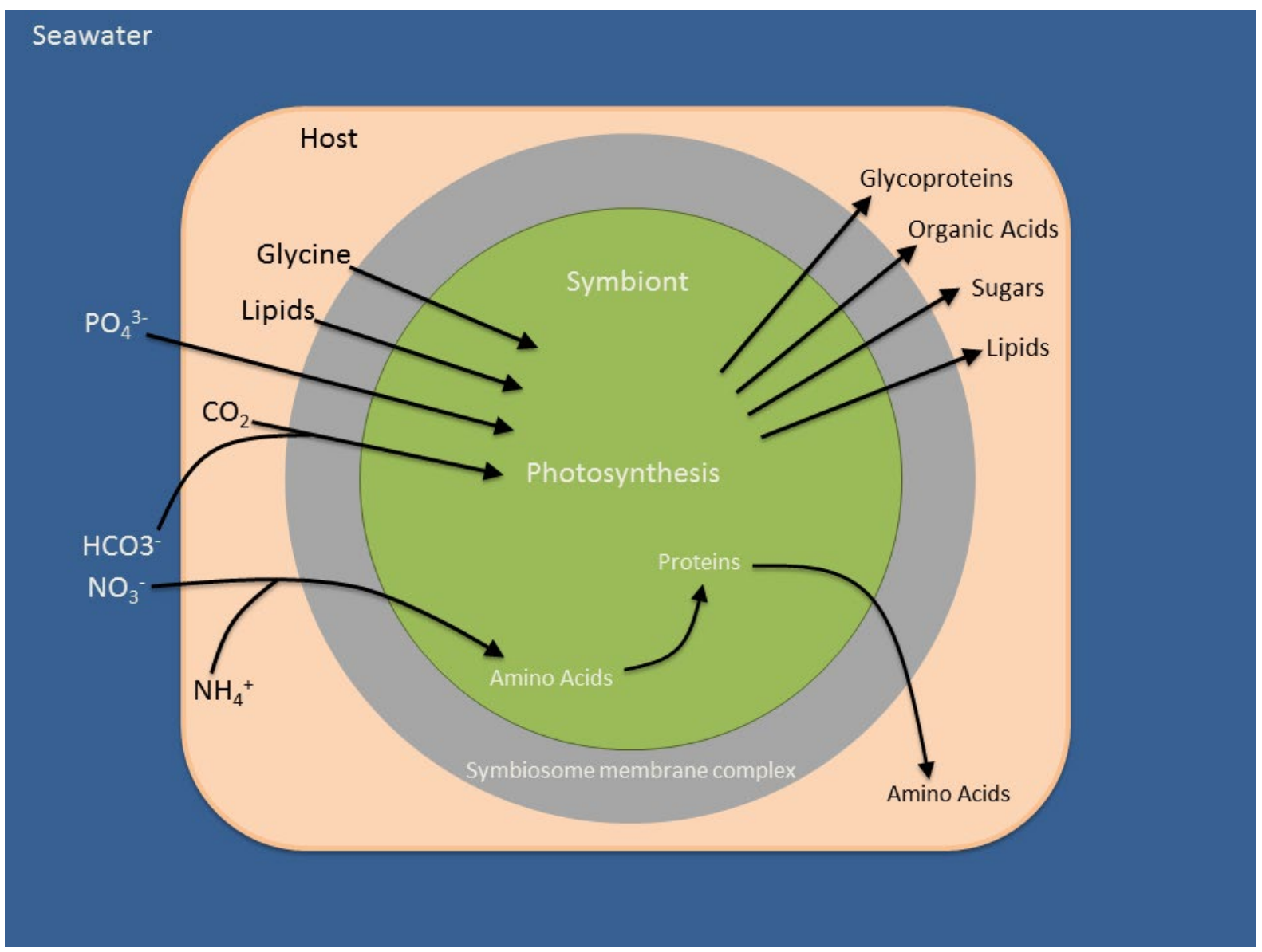

Figure 1.3. Metabolic interactions in the cnidarian-dinoflagellate symbiosis. Bicarbonate, nitrate and phosphate are translocated from ambient seawater to the algal partner. Carbon dioxide and ammonium from host metabolism, in addition to lipids, fatty acids and glycine, are also translocated to the host. The symbionts fix carbon during photosynthesis and provide the host with high energy molecules such as sugars. Note that seawater is shown completely surrounding the host cell for schematic purposes only.

\subsubsection{Symbiont Photosynthate}

The translocation of fixed carbon from symbiont to host has been the subject of a large number of studies (Muscatine and Cernichiari, 1969; Pupier et al., 2019; Ros et al., 2021; Trench, 1971). The algal partner uses light to fix carbon which can support more than $100 \%$ of the host's energy demands (Davies, 1984; Muscatine and Porter, 1977). Carbon in the form of carbon dioxide is primarily fixed by the $\mathrm{C} 3$ pathway of the Calvin cycle in Symbiodiniaceae (Streamer et al., 1993). Organic carbon-rich products can have different pathways and fates: 
they may either be metabolised by the algae, stored, or translocated to the host (Streamer et al., 1993). Initial studies suggested that the carbon is translocated to the host primarily in the form of glycerol (Muscatine, 1967, 1965). Nevertheless, more recent research has shown that glycerol is mostly released by symbionts in response to stress and forms only a minor component of the translocated carbon under non-stressful conditions (Suescún-Bolívar et al., 2016), while glucose is instead thought to be the primary form of fixed carbon translocated to the host (Burriesci et al., 2012; Hillyer et al., 2017b). Fatty acids such as palmitic, stearic, and oleic acid, and lipids like wax esters, triacylglycerol and sterols are also major players in the cnidarian-dinoflagellate symbiosis, as they are involved directly in the metabolism of each partner (Dunn et al., 2012; Hillyer et al., 2016; Yamashiro et al., 1999). Other products that are translocated to the host are organic acids, glycolic acids and amino acids (Fig. 1.3) (Trench, 1971; von Holt and von Holt, 1968; Whitehead and Douglas, 2003). Nevertheless, the identity and quantity of the mobile products released by the symbionts to the host remain uncertain due to complex biochemical pathways associated with this highly integrated endosymbiosis (Davy et al., 2012; Yellowlees et al., 2008).

\subsection{Mechanisms of Transport}

All mobile compounds, regardless of identity and origin, must traverse through the symbiosome membrane complex. In an attempt to identify symbiosome transporter proteins, Peng and co-workers isolated the symbiosome membrane via density gradient centrifugation of a holobiont homogenate. Subsequently, using 2D SDS-Page gel digestion, 17 protein groups were identified, of which the ATP-binding cassette (ABC) transporter was the only transmembrane protein (Peng et al., 2010). Additionally, in other studies vacuolar type proton pump (VHA) and sterol transporters proteins (i.e. NPC1 and NPC2) were localized to the symbiosome membrane complex (Barott et al., 2015b; Dani et al., 2017). Currently, very little is known about the transporter proteins of the symbiosome membrane surface, but generally there are three main classes of membrane proteins - channel proteins, transporters and pumps - that transport molecules and ions across cellular membranes (Alberts, 2017; Lodish et al., 2016). All transport proteins are embedded in the membrane. These proteins allow hydrophilic substances to traverse the membrane by forming a protein-lined pathway across it and therefore preventing any contact with its hydrophobic interior (Lodish et al., 2016). 


\subsubsection{Channel Proteins}

Channel proteins, also referred to as porins, form a hydrophilic passageway across the membrane through which multiple water molecules or ions can move simultaneously (Alberts, 2017; Lodish et al., 2016). Water, specific ions, or hydrophilic small molecules cross membranes through channels down their concentration or electric potential gradients without consuming energy (Finazzi et al., 2015; Lodish et al., 2016; Pizzagalli et al., 2021). Porins have the fastest transport rate $\left(10^{7}-10^{8}\right.$ ions s$\left.^{-1}\right)$ (Finazzi et al., 2015; Lodish et al., 2016). Some channels are gated and open only in response to specific chemical or electrical signals, while other types of channels are typically open much of the time (Fig. 1.4) (Alberts, 2017; Lodish et al., 2016). Channels, like all transport proteins, can be very selective for the type of molecule they transport (Lodish et al., 2016; Park and Saier, 1996). In the cnidarian-dinoflagellate symbiosis, aquaglyceroporins increase in abundance as a result of photoinhibitory stress (Yuyama et al., 2012). Aquaglyceroporins are members of a larger polyphyletic water channel aquaporin family (AQPs) (Frøkiær et al., 2009). AQP3, AQP7 and AQP9 are able to transport glycerol in addition to water (Carbrey et al., 2003; Thomas et al., 2002). AQPs have been detected in genomic and transcriptomic studies of cnidarian-dinoflagellate symbioses (Baumgarten et al., 2015; Lehnert et al., 2014). Moreover, in a recent bioinformatic comparison of cnidarian and human AQP sequences, Sproles et al (2018) predicted that AQP9 in cnidarians is only localised to the symbiosome membrane and might function as a glycerol channel. Nevertheless, experimental evidence is required to fully confirm what molecules are transported by cnidarian channel proteins.

\subsubsection{Transporters}

Transporters, also called carriers, have a slower transport rate $\left(10^{2}-10^{4}\right.$ molecules/s $)$ than channels, as these carriers are more specific (Lodish et al., 2016). After binding to solutes, a series of conformational changes take place that subsequently expose solute-binding sites on the other side of the membrane (Alberts, 2017). A wide variety of ions and molecules are transported by these carriers (Lodish et al., 2016). Three types of transporters have been identified: (1) uniporters, which translocate a single type of molecule down their electrochemical gradient; (2) symporters and (3) antiporters, in which transfer of the desired 
solute strictly depends on the transport of a second solute down its gradient, directly or indirectly, respectively (Fig. 1.4) (Alberts, 2017; Lodish et al., 2016).

A

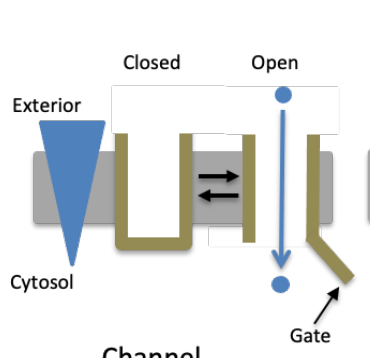

Channel

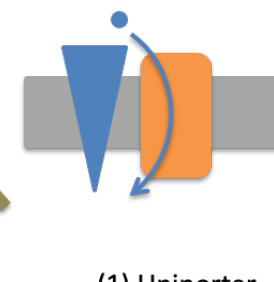

(1) Uniporter
B

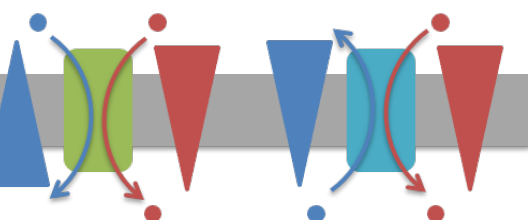

(3) Antiporter
C

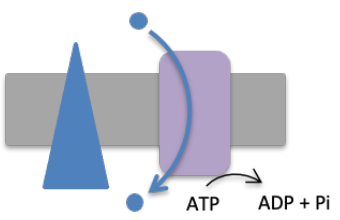

ATP Pump

Figure 1.4. Overview of transmembrane proteins. Electrical or concentration gradients are shown by triangles pointing from high to low. (A) Channels letting molecules through or water down the gradient. (B) Transporters which facilitate translocation across the membrane using energy stored in an electrical or concentration gradient. (B1) Uniporters transporting molecules down the electrochemical gradient. Cotransporters: (B2) Symporter and (B3) Antiporter, catalysing movements of molecules against the gradient by harvesting energy stored in electrochemical gradients of other molecules. (C) ATP Pumps, hydrolysing ATP to move specific molecules against the electrochemical gradient.

Uniporters, such as glucose transporters (GLUTs) and sugar transporter (SWEETs), facilitate sugar translocation along a gradient in metazoans (Wood and Trayhurn, 2003), and homologs have been identified in Breviolum (family: Symbiodiniaceae) transcriptomes (Xiang et al., 2018, 2015), and cnidarian genomic and transcriptomic studies (Baumgarten et al., 2015; Kitchen et al., 2015; Lehnert et al., 2012; Putnam et al., 2007). In the case of symporters, sodium-dependent symport activity for glucose and glycerol uptake by freshly isolated Symbiodinium microadriaticum (McDermott and Blanquet, 1991), and $\mathrm{HCO}_{3}{ }^{-}$assimilation by cultured Symbiodiniaceae have been reported (Al-Moghrabi et al., 1996). A recent study investigating solute carriers (SLC) in the symbiotic scleractinian coral Stylophora pistillata, found SLC4, the human homolog of which is an anion antiporter (Rungroj et al., 2004) that has roles in calcification (Zoccola et al., 2015).

\subsubsection{Pumps}

ATPases or ATP-powered pumps use the energy of ATP hydrolysis to move ions or small molecules across a membrane against a chemical concentration gradient, an electric potential, or both (Alberts, 2017; Lodish et al., 2016). However, pumps are the slowest membrane proteins, with a transport rate of $10^{0}-10^{3}$ ions $\mathrm{s}^{-1}$. Similar to cotransporters that harness energy stored in an electrochemical gradient, ATPases couple energetically unfavourable 
transportation with ATP hydrolysis, which is an energetically favourable reaction (Fig. 1.4) (Alberts, 2017; Lodish et al., 2016). Gradients caused by some ATPase activity can sometimes be used to drive a variety of secondary active transport processes by symporters and antiporters (e.g. $\mathrm{H}^{+}$gradient in case of proton pumps) (Ip et al., 2018).

ATPases (e.g. ABC transporter or V-type $\mathrm{H}^{+}$ATPase) are perhaps the most well-studied symbiosome-associated transmembrane proteins in the cnidarian-dinoflagellate symbiosis (Barott et al., 2015b; Peng et al., 2010). Being reported from prokaryotes to humans, ABC transporters belong to the $\mathrm{ABC}$ superfamily, which is the largest and most diverse transmembrane transporter family (Higgins, 1992; Ter Beek et al., 2014). Normally, these proteins pump a wide range of substrates, including amino acids, sugars, inorganic ions, polysaccharides, peptides, and even proteins, across the membrane against a concentration gradient by using the energy of ATP hydrolysis (Higgins, 1992; Ter Beek et al., 2014). V-type $\mathrm{H}^{+}$ATPases are proton pumps which are only found on vacuole membranes. These V-type ATPases are abundantly expressed on host-derived symbiosome surfaces, where they acidify the symbiosome vacuole down to $\mathrm{pH} \sim 4$ by active translocation of protons as part of a CCM (Barott et al., 2015b).

Our knowledge about proteins involved in metabolic translocation across the symbiosome membrane is nascent and future research is needed to fully identify transporters and further decipher metabolic pathways (Davy et al., 2012; Rosset et al., 2021).

\subsection{Reef Ecosystem and Threats}

Coral reefs, which are primarily dependent on the endosymbiotic relationship between dinoflagellates and cnidarians (Richmond, 1993), occupy only $0.2 \%$ in area of the marine environment, however they are the most biodiverse ecosystem in the ocean, and are estimated to harbour around $34 \%$ of all described marine species (Reaka-Kudla, 2001). Complex threedimensional landscapes built by corals promote elaborate adaptation, richly complex species interdependencies, and even a fertile source of medically active compounds (Veron et al., 2009). They also form barriers along coastlines which protect lagoons and mangrove habitats, both of which are crucial for vulnerable life-stages of a broad range of commercial and non- 
commercial species (Coker et al., 2014; McCook et al., 2009). Furthermore, life for tens of millions of people depends on reef ecosystems for protein and other services (Kleypas et al., 2021; Veron et al., 2009).

The endosymbiosis between hermatypic corals and photosynthetic dinoflagellates is very susceptible to physical and chemical fluctuations in the environment (Hoegh-Guldberg et al., 2017), and longer-term environmental stresses can result in a significant decline in the density of endosymbiont cells and/or the concentration of photopigments, in a process known as coral bleaching (Hoegh-Guldberg et al., 2007; Hughes et al., 2003; Marshall and Schuttenberg, 2006). Regrettably, locally and globally corals are challenged by anthropogenic activity, including coastal development, marine pollution and overexploitation, to the extent that at least $50 \%$ of tropical coral reefs have been lost over the past 50 years (Hoegh-Guldberg et al., 2017). The frequency and scale of mass coral bleaching events have escalated since the early 1980s (Baker et al., 2008; Glynn, 1993; Hoegh-Guldberg et al., 2007; Hughes et al., 2018) due to rapid increases in the atmospheric carbon dioxide concentration, which is leading to global warming and ocean acidification; this is threatening the survival of coral reefs beyond the end of the current century (Hoegh-Guldberg et al., 2007; Kleypas et al., 2021). More importantly, the consequences of coral reef destruction are not just limited to socioeconomic losses through impacts on fisheries, tourism and other ecosystem services, but likely also involve the extinction of a large part of the Earth's total biodiversity to a degree that has never happened before in human history (Veron et al., 2009).

\subsection{Diversity and Specificity of Symbionts}

\subsubsection{Diversity of Symbionts}

Algal symbionts living with the cnidarian host were first isolated from the scleractinian coral Cladocora caespitosa and the upside-down jellyfish Cassiopeia borbonica in a study on chlorophyll significance in animals, and subsequently were described as Zooxanthellae, meaning yellow animal cells (Brandt, 1882; Krueger, 2017). After this, the taxonomy of Zooxanthellae was confusing for a long time as numerous genera were described from different marine invertebrates (see Blank and Trench, 1986). Then, Kawaguti isolated coccoid symbionts from the reef coral Acropora corymbosa, which became motile gymnodinioid swarms in culture, and described them as the dinoflagellate genus Gymnodinium (Kawaguti, 
1944). Nevertheless, this terminology was abolished by subsequent studies, and Freudenthal officially described the genus Symbiodinium, meaning living together, from the upside-down jellyfish (Freudenthal, 1962). Indeed, eight genera in four or five orders of dinoflagellates were identified afterwards as being endosymbiotic with marine invertebrates and protists (Banaszak et al., 1993; Trench, 1997), of which Symbiodinium was the most studied genus. This genus was commonly found in cnidarians inhabiting shallow-water tropical and subtropical regions. Symbiodinium was reported from members of different classes of Cnidaria, including Anthozoa (e.g. anemones, scleractinian corals, black corals, zoanthids, corallimorphs, blue corals, alcyonacean corals, and sea fans), Scyphozoa (e.g. rhizostome and coronate jellyfish) and Hydrozoa (e.g. milleporine fire corals) (Baker, 2011). S. microadriaticum was initially considered to be a single ubiquitous species within the genus (Trench, 1979) until three new species, S. pilosum, S. goreauii and S. kawagutii, were described from the zoanthid Zoanthus sociatus, the sea anemone Ragactis lucida and the stony coral Montipora verrucosa, respectively (Trench and Blank, 1987).

Nonetheless, the identification of Symbiodinium species and subspecies by using morphological characteristics (as were used in these early studies) could not practically classify this divergent group. Hence, with the advent of molecular techniques, investigations on the phylogeny of Symbiodinium from reef corals using DNA markers started (Rowan and Powers, 1991a, 1991b). First, using the small ribosomal subunit, three genetic clades (A-C) were reported (Rowan and Powers, 1991b). In subsequent research, eight clades and numerous subclades ('types') were reported by using newer markers and techniques, such as ribosomal internal transcribed spacers (ITS) and denaturing gel gradient electrophoresis (DGGE) (LaJeunesse, 2001; Loh et al., 2001; Pochon et al., 2001; Pochon and Gates, 2010). In addition, new species of Symbiodinium were subsequently reported (e.g. S. thermophilum and $S$. trenchii), the descriptions of which were robustly supported by a combination of nuclear, mitochondrial and chloroplastic DNA markers (Hume et al., 2015; LaJeunesse et al., 2014). Nevertheless, a relatively recent systematic revision of the genus Symbiodinium formally described some of the "clades" as different genera within the family Symbiodiniaceae while retaining previously described species within each new genus (LaJeunesse et al., 2018). Thus, eleven genera have been officially described to date (Fig. 1.5) (LaJeunesse et al., 2021, 2018; Nitschke et al., 2020; Pochon and LaJeunesse, 2021). 


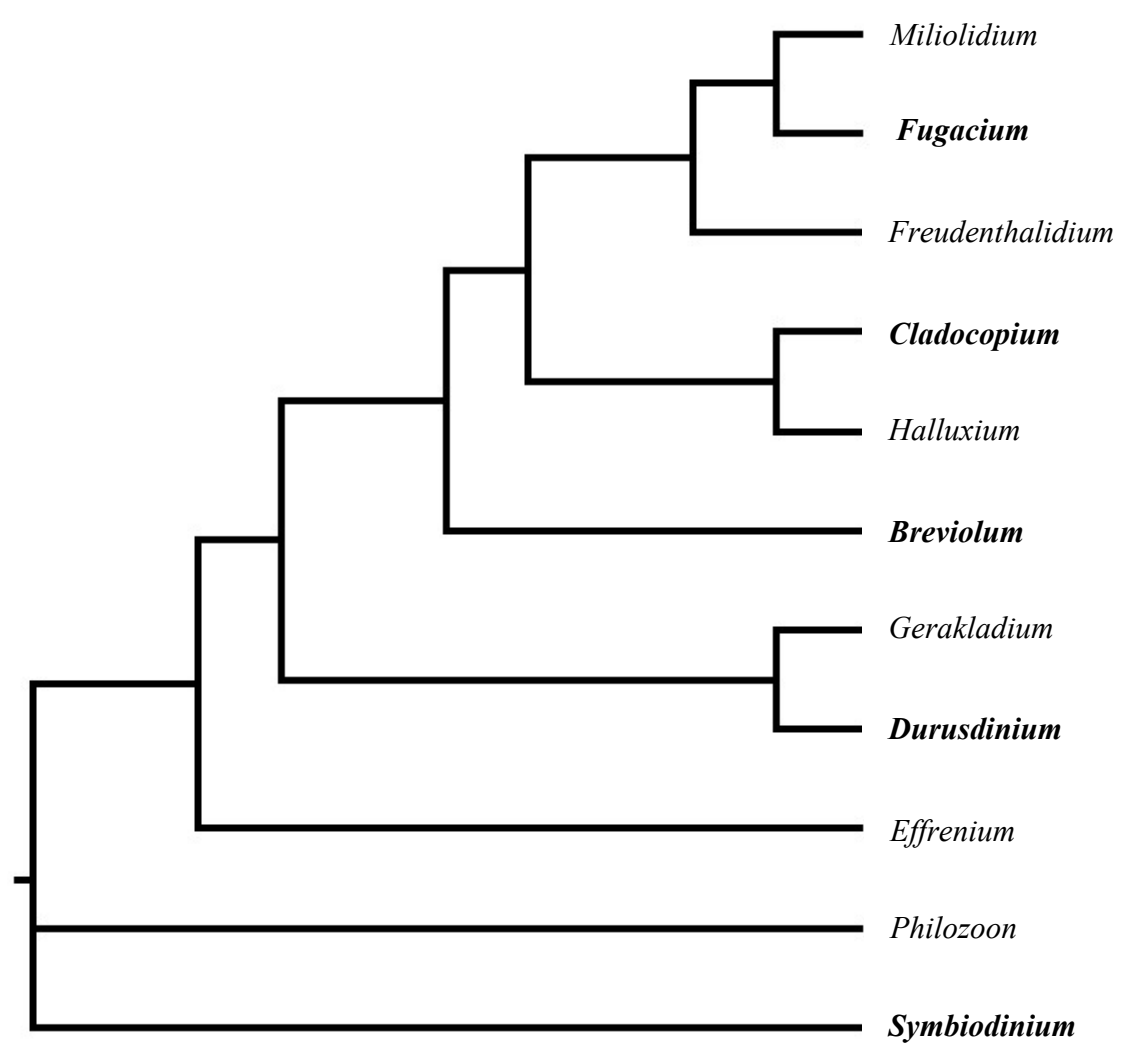

Figure 1.5. Phylogenetic relationships between officially described genera of Symbiodiniaceae to date based on internal transcribed spacer 2 genomic DNA sequences. Bold font shows those genera associated with scleractinian corals.

Nutrient assimilation, photosynthetic performance and symbiotic success rate can also be different between Symbiodiniaceae species or genera (Baker, 2003; McIlroy et al., 2016; Starzak et al., 2014; Stat et al., 2009; Thornhill et al., 2014). Thus, physiological and ecological features of Symbiodiniaceae taxa can further affect the holobiont's tolerance under different environmental regimes and stresses (Bellantuono et al., 2012; Fisher et al., 2012; Franklin et al., 2004; LaJeunesse et al., 2009; Rowan et al., 1997; Rowan and Knowlton, 1995; Stat and Gates, 2010; Tchernov et al., 2004). For instance, Durusdinium trenchii has been reported to be stress-tolerant (LaJeunesse et al., 2014), species of Symbiodinium (formerly known as clade A) are adapted to high irradiance (Grégoire et al., 2017; Reynolds et al., 2008) and Cladocopium thermophilum (formerly known as subclade C3 Gulf), which is widely prevalent in southern parts of the Persian Gulf (PG), is the most heat-tolerant species known in the family (D'Angelo et al., 2015; Hume et al., 2015). In contrast, some species from the genus Breviolum (formerly known as clade B) are adapted to tolerate cold stress (Thornhill et al., 2009). Some genera are even free-living (Effrenium) or only form symbioses with marine 
invertebrates other than cnidarians (e.g. Miliolidium) (Carlos et al., 1999; Hansen and Daugbjerg, 2009; Pawlowski et al., 2001; Pochon and LaJeunesse, 2021).

Although intensive studies on identification, genetic structure and corresponding ecological distributions of the members of the family Symbiodiniaceae have significantly increased our understanding of the cnidarian-dinoflagellate symbiosis, more research on the differences in cellular function/dysfunction of Symbiodiniaceae are the next steps for understanding the potential for acclimatisation, adaptation and survival of our invaluable coral reefs.

\subsubsection{Symbiosis Specificity}

Symbiosis specificity is defined as the taxonomic range of partners that can be involved in the association (Baker, 2003; Wang et al., 2012). Specificity in symbiosis is a result of coevolution between partners to generate an optimal symbiotic relationship (Thrall et al., 2007). Cnidariandinoflagellate symbioses also demonstrate high specificity, in particular in brooder species which obtain their symbionts maternally (Trench, 1997). For instance, host specialist Symbiodiniaceae are necessarily found in association with their desired host, while some other Symbiodiniaceae are found in a variety of cnidarian hosts and hence are considered as host generalists or even opportunists (e.g. Cladocopium goreauii or D. trenchii) (Finney et al., 2010; LaJeunesse, 2005; LaJeunesse et al., 2014, 2010; Thornhill et al., 2014). Molecular studies in recent years have shed light on host-symbiont specificity in nature (Arif et al., 2014; DíazAlmeyda et al., 2017; LaJeunesse, 2002; Silverstein et al., 2011; Thornhill et al., 2014; Wham et al., 2017; M. Ziegler et al., 2017), including its interaction with annual or post-bleaching dynamics of symbiont populations (D'Angelo et al., 2015; Hoegh-Guldberg et al., 2002; Hume et al., 2013, 2015; Lewis and Coffroth, 2004; Riegl et al., 2012). Nevertheless, coevolution has also resulted in host preference for certain symbiont types, as some cnidarian hosts harbour specific populations or population assemblages of Symbiodiniaceae (Baker, 2003; Trench, 1997). For example, non-native symbionts usually have a less successful rate of host colonisation than native algae (Davy et al., 1997; Gabay et al., 2019, 2018; Pettay et al., 2015; Starzak et al., 2014; Weis et al., 2001). Thus, symbiosis success differs with respect to the underlying mechanisms for symbiont acquisition, establishment and regulation (see Section 1.2.1 for details). 


\subsubsection{Adaptive Bleaching}

Buddemeier and Fautin (1993) hypothesised the 'adaptive bleaching mechanism', where reef corals, whether harbouring host-generalist or host-specialist symbionts, can use bleaching events as an opportunity to adapt to the surrounding environment. The hypothesis suggests that, following bleaching, the host can be repopulated by different, more thermally tolerant algal partners that can perform better under the stressful conditions. This can happen either via 'switching', acquiring symbionts from the environment; or 'shuffling', changing the abundance of existing symbiont communities within colonies (Baker, 2003) (Fig. 1.6).

\section{A}
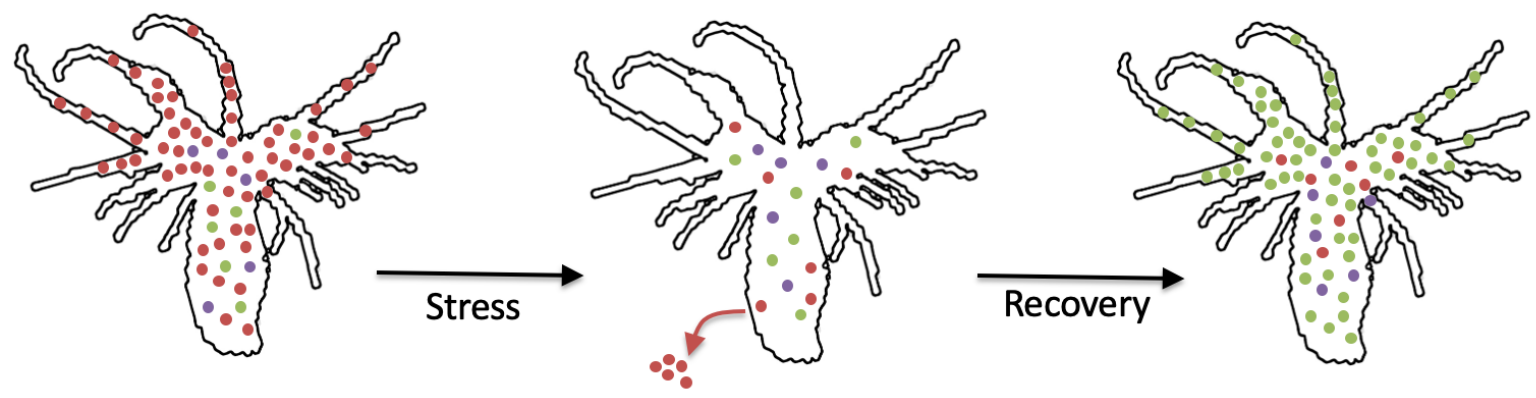

B
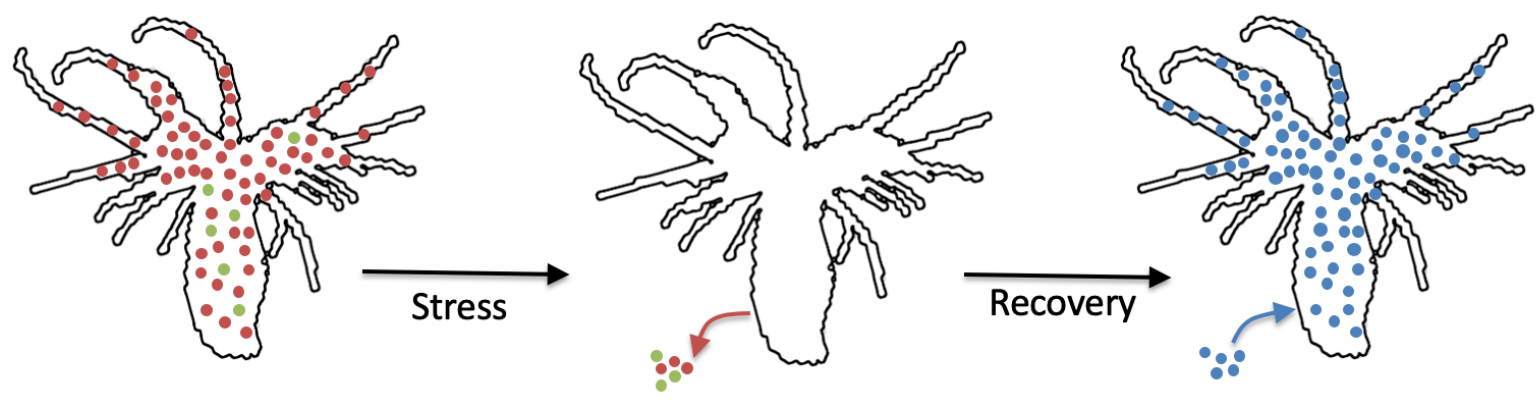

Figure 1.6. Adaptive bleaching mechanisms. (A) Shuffling, where there is a shift in the dominant member(s) of the existing symbiont community after bleaching or thermal stress. (B) Switching, where a brand new symbiont population is obtained from the surrounding environment after a bleaching event.

Symbiont shuffling has been reported in reef corals after bleaching (Kemp et al., 2014; LaJeunesse et al., 2009; Pettay et al., 2015). For example, D. trenchii is a host generalist and heat-tolerant member of the genus Durusdinium, and so is generally found in a variety of hosts, regardless of depth, particularly after mass bleaching events (Finney et al., 2010; Kemp et al., 2014; LaJeunesse et al., 2010). Nonetheless, members of this genus are not always optimal 
symbionts under normal conditions (Baker et al., 2013; Little et al., 2004). In addition, two recent studies have also shown shuffling and switching experimentally and naturally (Boulotte et al., 2016; Reich et al., 2017). Boulotte and co-workers provided evidence for switching to thermally resistant Durusdinium in two species of pocilloporid corals after two consecutive bleaching, however, evidence showing that switching is happening naturally in corals in the field are scarce.

\subsection{Cnidarian-Dinoflagellate Symbiosis in "Omics" Era}

In the last two decades, every discipline in biology and biomedicine has progressed substantially due to "omics" technology. "Omics" technology provides high throughput data that allow for a comprehensive description and comparison of almost all components within the cell; for instance genomics, transcriptomics, metabolomics and proteomics. Of these, proteomics is considered as the most significant technology, as it integrates changes in gene expression, mRNA stability, and protein post-translational modification (PTM) and turnover, in response to environmental change (Slattery et al., 2012; Tomanek, 2014). Proteomic approaches represent the interface between genotypic and phenotypic variation, and therefore the proteome is considered a key biomarker in eco-physiological acclimatisation and adaptation responses (Slattery et al., 2012).

The first complete genome sequence of a cnidarian was for the non-symbiotic Nematostella vectensis (Putnam et al., 2007). Currently, four genomes and 28 transcriptomes of cnidarians, and at least one genome and five transcriptomes from Symbiodiniaceae are publicly available as a result of efforts during the past decade (Meyer and Weis, 2012; Sproles et al., 2018). These datasets have been used to infer more information and provide a foundation for future investigations. For example, the non-symbiotic Nematostella genome made identification of cell-surface recognition mechanisms possible (Wood-Charlson and Weis, 2009). In addition, proteins that are involved in symbiosis and stress have been identified and quantified by obtaining proteomic profiles of symbiotic and aposymbiotic (i.e., symbiont-free) Aiptasia (Oakley et al., 2017, 2016; Sproles et al., 2019) using the genomic database provided by Baumgarten et al. (2015). In addition, metabolite profiles of both host and symbionts from Aiptasia and the hermatypic coral Acropora aspera during thermal stress and bleaching have been obtained by Hillyer et al. $(2017,2016)$ and Matthews et al. $(2018,2017)$. These findings 
have furthered our understanding of the metabolic changes during thermal bleaching, and have kick-started the field of coral metabolomics. These are just few examples of recent studies that were made feasible by "omics" technologies, and on which future studies can build.

Recent advances have been facilitated by immunocytochemistry (ICC). This is the process of using antibodies for demonstrating the presence of a particular antigen (usually peptides, proteins or polysaccharides) in a cell. ICC provides unique detection of proteins and their location (immunolocalization) by harnessing the high binding specificity of antibodies. Antibodies are proteins that are generated by $\mathrm{B}$ cells in response to foreign molecules (antigens), and hence are also called immunoglobulins (Ig). Antigens can be either extracted and purified proteins of interest, or synthetic peptides based on a known protein sequence. Out of six classes of immunoglobulin, IgG is regularly used for ICC. IgG has high specificity for its antigens. After attachment of IgG (primary antibody) to the antigen, it needs to be visualised by using appropriate reporter labels. Labels can be different for example IgG itself can be conjugated to a fluorescent dye, however usually another fluorescently-labelled antibody (secondary antibody), one that recognises and binds to a specific constant domain of the primary antibody, is used, (Fig. 1.7) (Burry, 2009; Hoppert and Wrede, 2011; Renshaw, 2017; Tiller and Tessier, 2015). ICC techniques have been used in the cnidarian-dinoflagellate field before. For instance, the protein Sym32 was immunolocalized to the symbiosome membrane and also in an aposymbiotic host (Schwarz and Weis, 2003). Using immunolocalization techniques coupled with transmission electron microscopy (TEM) analysis, it was hypothesised that the outermost symbiosome membrane bilayer is derived from the host (Wakefield and Kempf, 2001). Other examples include the use of ICC for identification of symbiont exudates in culture and in hospite (Markell and Wood-Charlson, 2010). More recently, Barott and co-workers localised V-type $\mathrm{H}^{+}$ATPase on the symbiosome membrane in symbiosis (Barott et al., 2015b). In addition, a recent study visualised different NPC2 proteins in the symbiotic sea anemones Anemonia viridis and Aiptasia, one of which was only present in the symbiotic state and was closely associated with the symbiosome membrane complex (Dani et al., 2017, 2014). 
A

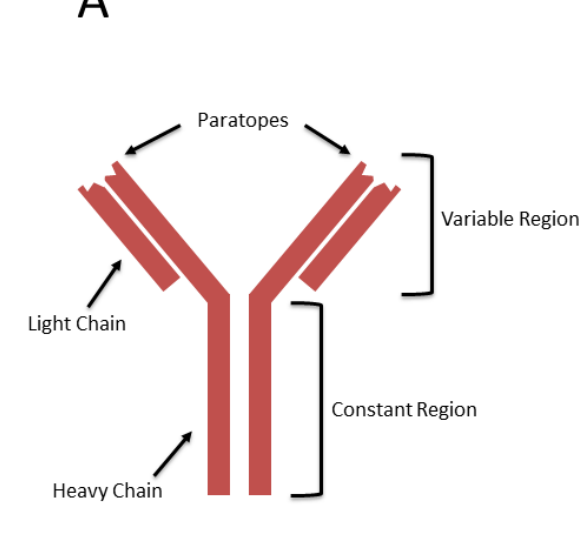

B

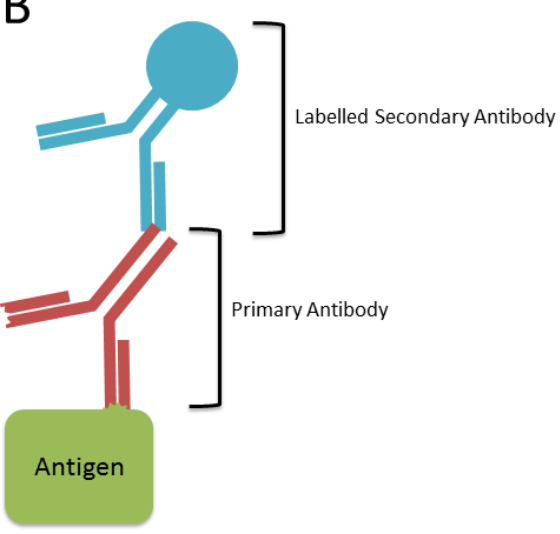

Figure 1.7. Antibodies and their application in immunocytochemistry (A) Structure of an IgG class antibody showing antigen-binding highly variable regions (Paratopes) on variable region. (B) Fluorescent label conjugated to secondary antibody used for immunolocalization.

\subsection{Model System}

In spite of a large number of studies investigating the phylogenetic diversity, population structure, ecological distribution, and role of Symbiodiniaceae with respect to environmental stressors, including climate change (Hoegh-Guldberg et al., 2002; Hume et al., 2015; LaJeunesse et al., 2014, 2004, 2003; Pochon and Gates, 2010; Rowan and Powers, 1991a), our fundamental understanding of the cellular processes in the cnidarian-dinoflagellate symbiosis remains poor. Indeed, there are still considerable gaps in our knowledge of how the symbiosis is established and maintained, and conversely what causes it to become dysfunctional at the cellular level (Davy et al., 2012). Model system investigations have been extensively used in molecular, cell and developmental biology, from prokaryotes to mammals and angiosperms (Davis, 2004). For a long time, Hydra has been a model cnidarian in biology, however only one species in the genus has an endosymbiotic relationship with green algae, belonging to the genus Chlorella (Galliot, 2012). In addition, corals are hard to maintain in the laboratory for cellular experiments, as their skeleton, low growth rate and specific environmental needs are limiting (Weis et al., 2008). As model organisms must be well-chosen so that results can be directly comparable (Weis et al., 2008), Exaiptasia pallida, (Fig. 1.8) (Grajales and Rodríguez, 2014), has been proposed as a model for cellular studies of the cnidarian-dinoflagellate symbiosis (Weis et al., 2008). This is especially important given current interest in how symbiont diversity might allow coral reefs to adapt to climate change and hence impact the sustainability of reefs. This sea anemone can be grown in large numbers rapidly and is able to be rid of its symbiotic algae and infected with new types, therefore providing an opportunity 
not only to examine basic symbiosis function, but also the effect of symbiont type on this function. Aiptasia has been extensively used for such studies (Bucher et al., 2016; Gabay et al., 2019, 2018; Hillyer et al., 2016; Matthews et al., 2017, 2016; Parkinson et al., 2018; Rädecker et al., 2017b; Starzak et al., 2020, 2014; Wolfowicz et al., 2016), and genomic, transcriptomic and proteomic data are already available (Baumgarten et al., 2015; Lehnert et al., 2012; Oakley et al., 2017, 2016; Sproles et al., 2019). This invaluable information provides a foundation for future in-depth cellular studies.

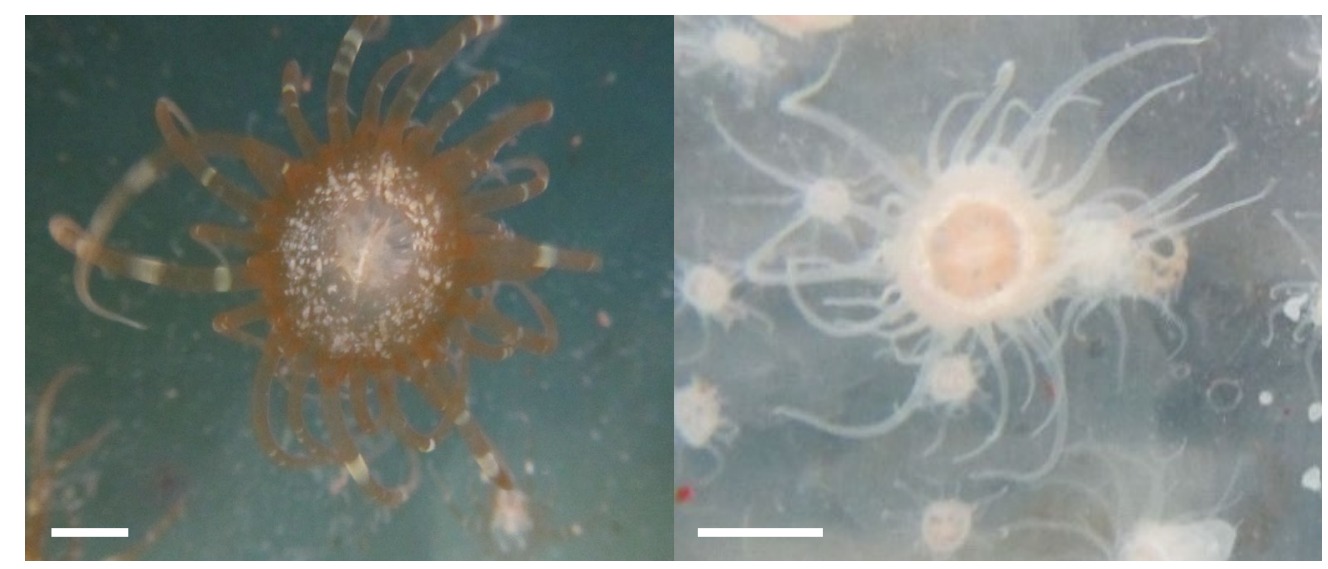

Figure 1.8. Sea anemone Exaiptasia pallida, commonly known as 'Aiptasia'. Symbiotic (left) and aposymbiotic (right) Aiptasia. Scale bar is $5 \mathrm{~mm}$.

\subsection{Aims and Objectives}

The primary aim of this thesis was to determine the impact of partner switching on the function of the symbiont and host-symbiont interface, to elucidate the potential for corals and other cnidarians to adapt to warming oceans through acquiring new symbiotic partners. This aim was addressed with the Aiptasia, model system, combined with the latest molecular and 'omics' approaches, with an emphasis on proteomics and immunocytochemistry. The specific objectives of this $\mathrm{PhD}$ research were:

1. To compare and contrast the proteome of the native symbiont (B. minutum) when ex and in hospite with the Aiptasia.

Hypothesis: The proteome of $B$. minutum will change significantly in symbiosis, reflecting the functional needs of this lifestyle. 
2. To compare and contrast the proteomes of different Symbiodiniaceae species when in symbiosis with Aiptasia, both in the early and later stages of symbiosis establishment.

Hypothesis: The proteome of the different symbiont species will differ over time, reflecting differential capacities to form a symbiosis.

3. To immunolocalise symbiosome membrane complex-associated transporter proteins in the cnidarian-dinoflagellate symbiosis, and measure how expression of these proteins differs in different host-symbiont pairings.

Hypothesis: The symbiosome membrane complex includes modified proteins to facilitate nutrient and photosynthetic product exchange, and the expression of these proteins is influenced by symbiont type.

Note: The research section of this thesis has been written as a series of stand-alone manuscripts (Chapters 2-4), therefore some overlap occurs between chapters, especially in the introductions. 


\section{Chapter 2 \\ The influence of symbiosis on the proteome of the symbiotic dinoflagellate Breviolum minutum}

\subsection{Introduction}

Reef ecosystems are formed by an obligatory endosymbiotic relationship between cnidarians and dinoflagellate symbionts from the family Symbiodiniaceae. This symbiotic relationship is hugely important in oligotrophic seas, where corals are major primary producers and provide a three-dimensional landscape to promote the abundance and diversity of other species (Veron et al., 2009). Nutritional exchange is integral to the cnidarian-dinoflagellate association, with the host being responsible for providing shelter and essential nutrients, such as inorganic carbon, nitrogen and phosphorus, to the algal symbionts. In return, the symbionts support host energy requirements by providing photosynthetic products (Hillyer et al., 2017b; Matthews et al., 2017; Muscatine and Porter, 1977). Glucose is the dominant form in which photosynthetically-fixed carbon is translocated to the host (Burriesci et al., 2012; Hillyer et al., 2017b; Suescún-Bolívar et al., 2016). Additionally, essential amino acids, fatty acids such as palmitic, stearic and oleic acids, and lipids such as wax esters, triacylglycerol and sterols, are translocated (Dunn et al., 2012; Hillyer et al., 2016; Wang and Douglas, 1999; Yamashiro et al., 1999).

The Symbiodiniaceae are physiologically and ecologically diverse, with eleven genera described to date (LaJeunesse et al., 2021, 2018; Nitschke et al., 2020; Pochon and LaJeunesse, 2021). When in hospite, the symbionts are located in a host-derived late arrested phagosome known as the symbiosome (Fitt and Trench, 1983; Hohman et al., 1982; Neckelmann and Muscatine, 1983; Wakefield et al., 2000; Wakefield and Kempf, 2001). The physiology, morphology and cell cycle of the symbiotic dinoflagellates has long been known to change in response to this symbiotic state (i.e. in hospite vs. ex hospite) (Trench, 1993; Trench and Blank, 1987). For example, when in symbiosis, the symbionts exist in coccoid form, lacking flagella and having thinner cell walls, whereas the free-living dinoflagellates revert to a motile, gymnodinioid form with two flagella (Freudenthal, 1962; Trench, 1993; Trench and Blank, 1987; Wakefield et al., 2000). Moreover, in symbiosis the dinoflagellates release a substantial portion of their photosynthate to the host, whereas they typically release only a small fraction 
when free-living (Cook et al., 1988; Muscatine, 1967, 1965; Muscatine et al., 1983; Trench, 1974).

However, our fundamental understanding of the cellular and molecular mechanisms that underlie the establishment, maintenance and collapse of the cnidarian-dinoflagellate symbiosis remains limited (Davy et al., 2012), while there has been a tendency for studies to focus on events occurring in the cnidarian rather than the dinoflagellate partner (Bay and Palumbi, 2015; Hillyer et al., 2017b, 2017a; Lehnert et al., 2014, 2012; Matthews et al., 2018, 2017; Oakley et al., 2017, 2016; Sproles et al., 2019). Nevertheless, there has been effort to remedy this disparity in recent years, with transcriptomic, proteomic (SDS-PAGE) and lipidomic studies of symbiotic dinoflagellates revealing changes in transmembrane transport, photosynthesis, lipid metabolism, cell division, and signalling, associated with a shift to/away from a symbiotic life-style (Bellantuono et al., 2019; Chen et al., 2015; Maor-Landaw et al., 2020; Pasaribu et $a l ., 2015)$. However, we still have more to learn, with advances in various 'omics' technologies, including proteomics, providing an opportunity to progress the field substantially. Proteins represent the biochemical machinery by which organisms respond to their changing environment, and unlike transcripts, protein investigation provides insights into molecular phenotype and functional adaptations. Therefore, proteomics, the study of protein dynamics and interactions, is an ideal approach for investigating how marine organisms adapt to changes in their environment (Tomanek, 2014).

Here, I employed the latest, comprehensive shotgun proteomic methods (Schubert et al., 2017), to further elucidate the changes associated with the establishment of the cnidariandinoflagellate symbiosis, building substantially on earlier SDS-PAGE analysis (Pasaribu et al., 2015). Specifically, proteomic profiles of Breviolum minutum, a native symbiont of the sea anemone Exaiptasia pallida (commonly referred to as 'Aiptasia'), were compared between the in hospite and ex hospite symbiotic states. Aiptasia is a widely used model organism for studying the cnidarian-dinoflagellate symbiosis (Grajales and Rodríguez, 2014; Weis et al., 2008). In addition, comparisons were made under a range of nutrient environments/feeding regimes to allow for the fact that nutrient availability in enriched seawater medium is likely to be very different from that experienced inside the host's cells, where nitrogen supply especially is thought to be actively restricted by the host as a means of limiting symbiont growth (Cook 
et al., 1988; Wang and Douglas, 1998; Xiang et al., 2020). This comparative proteomic profiling was used to identify algal proteins and cellular pathways that allow the dinoflagellate symbiont to survive and proliferate in the endosymbiotic state, and ultimately facilitate a successful cnidarian-dinoflagellate symbiosis.

\subsection{Materials and Methods}

\subsubsection{Experimental organisms and design}

Breviolum minutum (strain FLAp2, originally from Long Key, Florida) was cultured in Guillard's f/2 medium (AusAqua Pty, SA, Australia) in artificial seawater (ASW) (Coral Pro Salt, Red Sea, New Zealand); this medium has a much greater nitrogen concentration than ASW, with nitrate concentrations of $883 \mu \mathrm{M}$ and $30 \mu \mathrm{M}$, respectively (Guillard, 1975; Patey et al., 2008). Specimens of Aiptasia were taken from a clonal laboratory stock $(n=200$; clonal strain ID: NZ1). Strain NZ1 is originally from the Indo-Pacific region and naturally hosts $B$. minutum only, though a culture of B. minutum from Aiptasia NZ1 was not available at the time of this experiment.

Algal identity, both in culture and in anemones, was confirmed by the standard protocol. Briefly, DNA was extracted using CTAB/phenol-chloroform (Baker et al., 2004), and the internal transcribed spacer (ITS2) region of ribosomal DNA amplified by PCR using ITSintfor2 and ITS2Rev2 as the forward and reverse primers, respectively (LaJeunesse and Thornhill, 2011; Thornhill et al., 2007). Amplicons were directly sequenced (Macrogen Inc., Seoul, South Korea), sequences aligned with Geneious Prime v. 2019.2.3 (Biomatters Ltd., Auckland, NZ), and a custom BLAST search carried out against Symbiodiniaceae ITS2 sequences in Geosymbio (Franklin et al., 2012).

Anemones and cultured B. minutum were immediately used for the experiment; and preacclimatization was not performed to reduce mortality, especially of the cultured algae in ASW. Free-living B. minutum cultures were divided into fresh ASW and f/2 treatments (250 $\mathrm{ml}$ uncapped flask, $\mathrm{n}=9$ per treatment). Likewise, anemones were divided into well-fed and starved treatments $(250 \mathrm{ml}$ uncapped containers, $\mathrm{n}=9$ per treatment, 10 anemones per 
container). The well-fed anemone treatment was fed five times per week with Artemia sp. nauplii and the starved anemones were not fed during the experiment. All treatment media (i.e. ASW and f/2) were changed every day. The experiment was run for 21 days.

All treatments were maintained under an irradiance of $100 \mu$ mol photons $\mathrm{m}^{-2} \mathrm{~s}^{-1}$ on $12 \mathrm{~h}: 12 \mathrm{~h}$ light:dark cycle at $25^{\circ} \mathrm{C}$. PAM fluorometry (Diving-PAM-II, Walz, Germany) was used to measure maximum quantum yield of photosystem II (maximal variable fluorescence $\left(F_{v}\right) /$ maximal fluorescence yield $\left(F_{m}\right)$ ) every three days, following a one-hour dark adaptation at the end of the daily light cycle, to assess the photosynthetic health of the algae. The measurement distance was a standard $5 \mathrm{~mm}$. All PAM settings were maintained over the course of the experiment: measuring light 4 , saturation intensity 4 , saturation width $0.6 \mathrm{~s}$, gain 2 , and damping 2. Mean $( \pm S E) F_{v} / F_{m}$ was calculated based on measurements from each sample $(n=$ 9 per treatment) at each time-point.

At the end of the experiment (Day 21), anemones from each treatment were pooled (5-7 anemones per replicate, $\mathrm{n}=9$ replicates per treatment) and rapidly frozen at $-80^{\circ} \mathrm{C}$. Anemones were homogenized using a tissue homogenizer at $4{ }^{\circ} \mathrm{C}$, and symbionts were separated from the host by centrifugation at $500 \times \mathrm{g}$ for $5 \mathrm{~min}$ at $4{ }^{\circ} \mathrm{C}$. The host fraction was used for total protein quantification by fluorometry with a Qubit Protein Assay Kit (ThermoFisher Scientific). The symbiont pellet was resuspended and washed at $4{ }^{\circ} \mathrm{C}$ with $500 \mu \mathrm{ASW}$, a $10 \mu \mathrm{l}$ subsample taken for cell quantification by a haemocytometer, and the remaining resuspension (490 $\mu 1)$ was pelleted again by centrifugation at $500 \times \mathrm{g}$ for $5 \mathrm{~min}$ at $4{ }^{\circ} \mathrm{C}$. Free-living algae (i.e., in $\mathrm{f} / 2$ and ASW) were also centrifuged at $500 \times \mathrm{g}$ in $50 \mathrm{ml}$ Falcon tubes at $4{ }^{\circ} \mathrm{C}$ and then snap-frozen at $-80^{\circ} \mathrm{C}$. All samples were stored at $-80{ }^{\circ} \mathrm{C}$ until analysis.

\subsubsection{Protein extraction}

Algal pellets were washed with $500 \mu \mathrm{l}$ cold HPLC grade water at $4{ }^{\circ} \mathrm{C}$ to remove salts and resuspended in $500 \mu 1$ 1\% SDC in HPLC grade water. The resuspension was passed five times through a 23 -gauge needle $(0.337 \mathrm{~mm}$ inner diameter $)$ to remove any remaining host cell debris (e.g., symbiosome membrane) or protein of host origin. All the samples were centrifuged again 
at $500 \mathrm{x}$ g for $5 \mathrm{~min}$ at $4{ }^{\circ} \mathrm{C}$ and the host-containing supernatant was discarded. The pellet was resuspended in $500 \mu \mathrm{l}$ of 5\% sodium dodecyl sulphate in HPLC grade water. All cells were homogenized with an ultrasonic homogenizer (Vibra-Cell ${ }^{\mathrm{TM}}$ Ultrasonic VCX 500). Total protein extraction was conducted by incubating homogenized cell samples at $85^{\circ} \mathrm{C}$ for 20 min with 1\% B-mercaptoethanol (BME). Hydrophobic pigments were removed by ethyl acetate phase transfer. A filter-aided sample preparation (FASP) method was used for removal of SDC, and overnight protein digestion was performed by trypsin. Digested peptides were centrifuged into a new sample tube. Formic acid with a final concertation of $1 \%$ was used to terminate trypsin activity and precipitate any remaining SDC. All the samples were then desalted as described in Oakley et al. (2016).

\subsubsection{LC-ESI-MS/MS}

Liquid chromatography - electrospray ionization - tandem mass spectrometry (LC-ESIMS/MS) was used for peptide analysis. $200 \mathrm{ng}$ of peptides per sample were separated by liquid chromatography (Ultimate 3000, Dionex) on a PepMap C18 column (\#160321, Thermo Scientific) at $35{ }^{\circ} \mathrm{C}$ with a non-linear gradient, from $96 \%$ to $50 \%$ buffer A $(0.1 \%$ formic acid $)$ to buffer B ( $80 \%$ acetonitrile, $0.1 \%$ formic acid) and a flow rate of $0.3 \mu$ for $90 \mathrm{~min}$. An Orbitrap Fusion Lumos Tribrid mass spectrometer (Thermo Scientific) was used to analyse hyphenated peptides, which were ionized by electrospray needle at $1.8 \mathrm{kV}$. An orbitrap with resolution of $120 \mathrm{k}$ and scan range of 375-1599 (m/z) was used for acquiring MS spectra. Sequencing was performed via higher energy collisional dissociation (HCD) fragmentation on the top 20 precursors, and the fragment ion spectra (MS/MS) then acquired in the linear ion trap with dynamic exclusion enabled for $60 \mathrm{~s}$. Thermo Xcalibur package (v4.3) was used to control LC-ESI-MS/MS instruments.

\subsubsection{Protein identification and quantification}

Andromeda algorithm in MaxQuant v1.6.12.0 (Cox et al., 2014) was used to search the tandem MS spectra against a custom protein database constructed from a B. minutum transcriptome (Parkinson et al., 2016), using standard genetic code for translation. A built-in contaminants database from MaxQuant was also coupled in the search. A maximum of two missed cleavages of trypsin digestion, a minimum of two matching peptides per protein, and a minimum peptide 
length of seven amino acids all with false discovery rate of 0.01 were required for peptide and protein validations. Variable modifications were set as $\mathrm{N}$-terminus carbamylation and oxidation of methionine, and fixed modifications were specified as cysteine carbamidomethylation. All spectra from in hospite treatments were also searched against Aiptasia protein sequence databases (Baumgarten et al., 2015) to verify that few contaminating host proteins were present.

\subsubsection{Statistical analyses}

Cell counts and maximum quantum yield data were checked for normality by the ShapiroWilk test. A two sample $t$-test was used to compare the densities of symbionts from in hospite treatments. A mixed two-way analysis of variance (ANOVA) was used to test for differences between the maximum quantum yields between different treatment groups and days, followed by a Bonferroni post hoc test to identify any pairwise differences. All physiological data analyses were conducted in R 4.0.3 (R Core Team, 2020).

The bioinformatics software Perseus v.1.6.13.0 (Tyanova and Cox, 2018) was used to remove known contaminant proteins and false identification. The remaining protein precursor intensity was $\log _{2}$-normalized and further used to compare and identify significantly different proteins between treatments. Principal component analyses (PCA) were plotted by ClustVis (Metsalu and Vilo, 2015). A recently described robust statistical test method, PolyStest integrated with Miss Test, was used for protein intensity comparisons. The false discovery rate and log-ratio thresholds were set to $\leq 0.05$ and $\leq \pm 0.7$, respectively (Schwämmle et al., 2020). All detected proteins were searched against the UniProtKB database using DIAMOND (Buchfink et al., 2014) with an E-value $\leq 1 \times 10^{-5}$. The top manually reviewed SwissProt matches were used to assign protein sequence IDs and annotations, unless no SwissProt matches were detected, in which case TrEBML matches were assigned. Any unmatched sequences were designated as hypothetical proteins. 


\subsection{Results and Discussion}

\subsubsection{Endosymbiont density and photobiology}

ITS2 sequencing confirmed the genetic identity of the algae in all treatments as B. minutum. However, symbiont cell densities of well-fed anemones were approximately 1.6-fold higher than starved anemones (two-sample $\mathrm{t}$ test, $\mathrm{t}(6.90)=3.34, p=0.013$; Fig. 2.1A). Moreover,

A
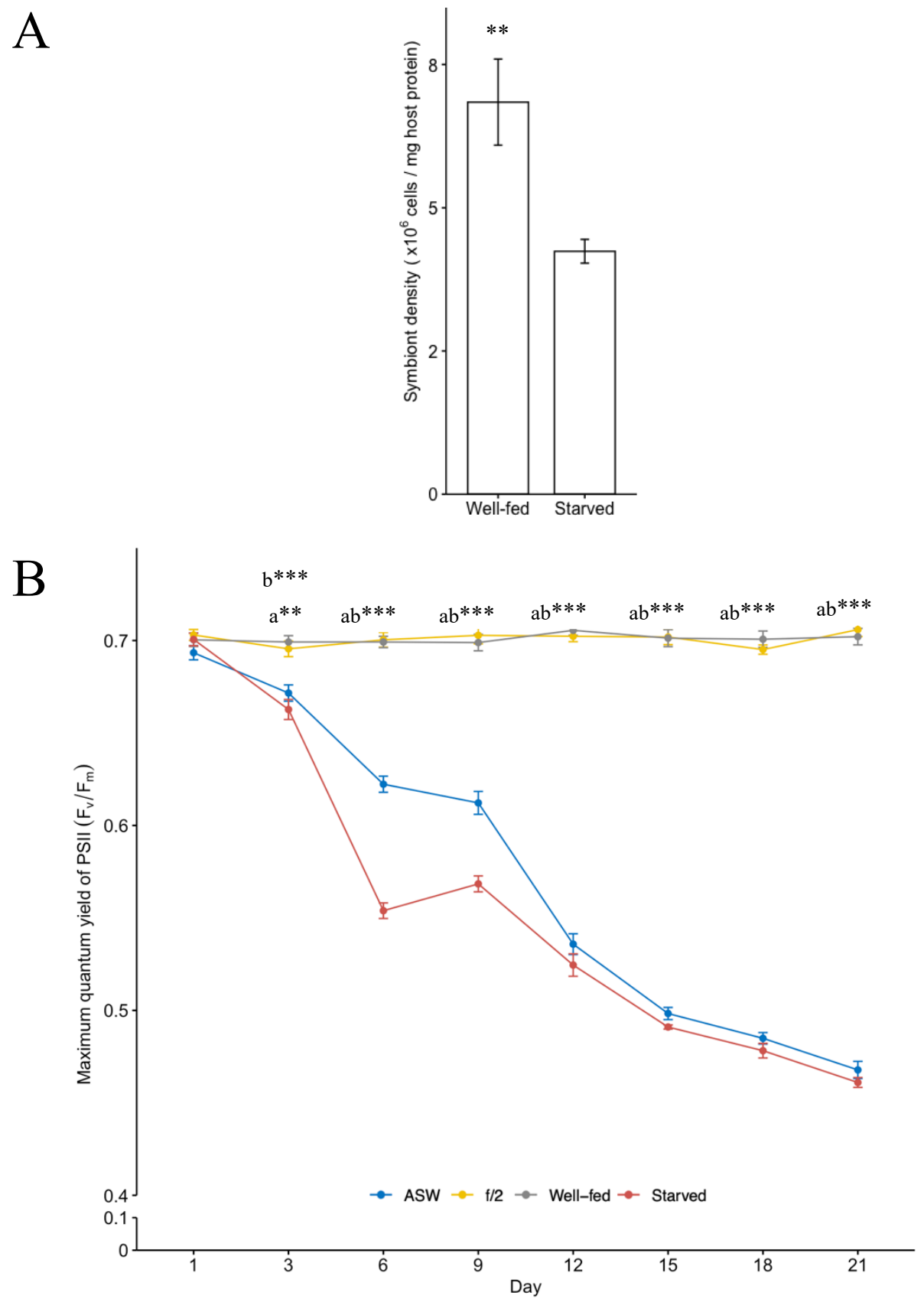

Figure 2.1. Physiological effects of different nutritional regimes in Breviolum minutum and Aiptasia. (A) Comparison of in hospite symbiont cell densities between well-fed and starved Aiptasia. $n=13$. (B) Maximum quantum yield of photosystem II (Fv/Fm) of cultured algae (ASW vs. f/2 medium) and in hospite (well-fed $v s$. starved). $\mathrm{n}=10$ per treatment at each timepoint. Asterisks indicate significant differences: ${ }^{*} \mathrm{p}<0.05 . * * \mathrm{p}<0.001,{ }^{* * *} \mathrm{p}<0.0001$. Different letters next to asterisks indicate significant differences between treatments, a (ASW and f/2), $b$ (well-fed and starved). Values are mean \pm SE. 
$\mathrm{F}_{\mathrm{v}} / \mathrm{F}_{\mathrm{m}}$ of cultured algae in ASW and in hospite algae in starved anemones declined significantly $(\sim 38 \%)$ from Day 3 onwards (two-way mixed ANOVA, $F_{7,252}=428.749, \mathrm{P}<0.001$ ), while algae in the nutrient-enriched $\mathrm{f} / 2$ and well-fed treatments showed no significant change in $\mathrm{F}_{\mathrm{v}} / \mathrm{F}_{\mathrm{m}}$ (Fig. 2.1B).

Decreases in symbiont density after prolonged starvation or under phosphate deficiency have been reported before (Rosset et al., 2017; Titlyanov et al., 2000). The host reduces nitrogen supply to the symbionts when starved, which subsequently results in nitrogen stress and reduced cell division (Muller-Parker et al., 1996). Moreover, the amount of chlorophyll per symbiont cell, cellular photosynthetic rate and chloroplast size decrease during nitrogen starvation (McAuley and Cook, 1994; Muller-parker et al., 1996). Thus, it is unsurprising that $\mathrm{F}_{\mathrm{v}} / \mathrm{F}_{\mathrm{m}}$ declined here in the low-nutrient treatments, regardless of symbiotic state.

\subsubsection{Proteins affected by symbiotic state and nutritional regime}

In general, symbiosis and nutritional state had considerable impacts on the B. minutum proteome, indicating a physiological response to living intracellularly in the acidic environment of the symbiosome (Barott et al., 2015b). Changes included: (1) modifications to cell wall assembly; (2) responses to prevent host phagosome fusion with the lysosome; (3) responses to carbon, nitrogen and iron excess/limitation; (4) protective measures against oxidative stress; and (5) changes in carbohydrate and fatty acid synthesis and metabolism (Tables 2.1 and 2.2).

A total of 2840 proteins were detected across all treatments, of which 56 known false matches and contaminant proteins were discarded. Principal component analysis plots depict protein expression as being distinctly different between symbiotic states and nutritional regimes (Fig. 2.2). A total of 1074 proteins were differentially expressed across all treatments (Table S2.1). A total of 1037 of these were matched based on homology with sequences in the UniProtKB database (E-value $\leq 1 \times 10^{-5}$ ), and the remaining unmatched 37 sequences were designated as hypothetical proteins. In other analyses, a total of 473 proteins were significantly different between the ex hospite (ASW and f/2 combined) and in hospite (well-fed and starved combined) states, and 684 proteins were differentially expressed between the high (well-fed and $\mathrm{f} / 2$ ) and low (starved and ASW) nutrient regimes (Tables S2.2 and S2.3). A total of 13 and 193 proteins were exclusively expressed in the symbiotic and free-living states, respectively. 
A total of 42 proteins were uniquely detected in both high nutrient regimes (well-fed and $\mathrm{f} / 2$ ), whereas only one protein was exclusively detected in both low nutrient regimes (starved and ASW). These are discussed further in section 2.3.
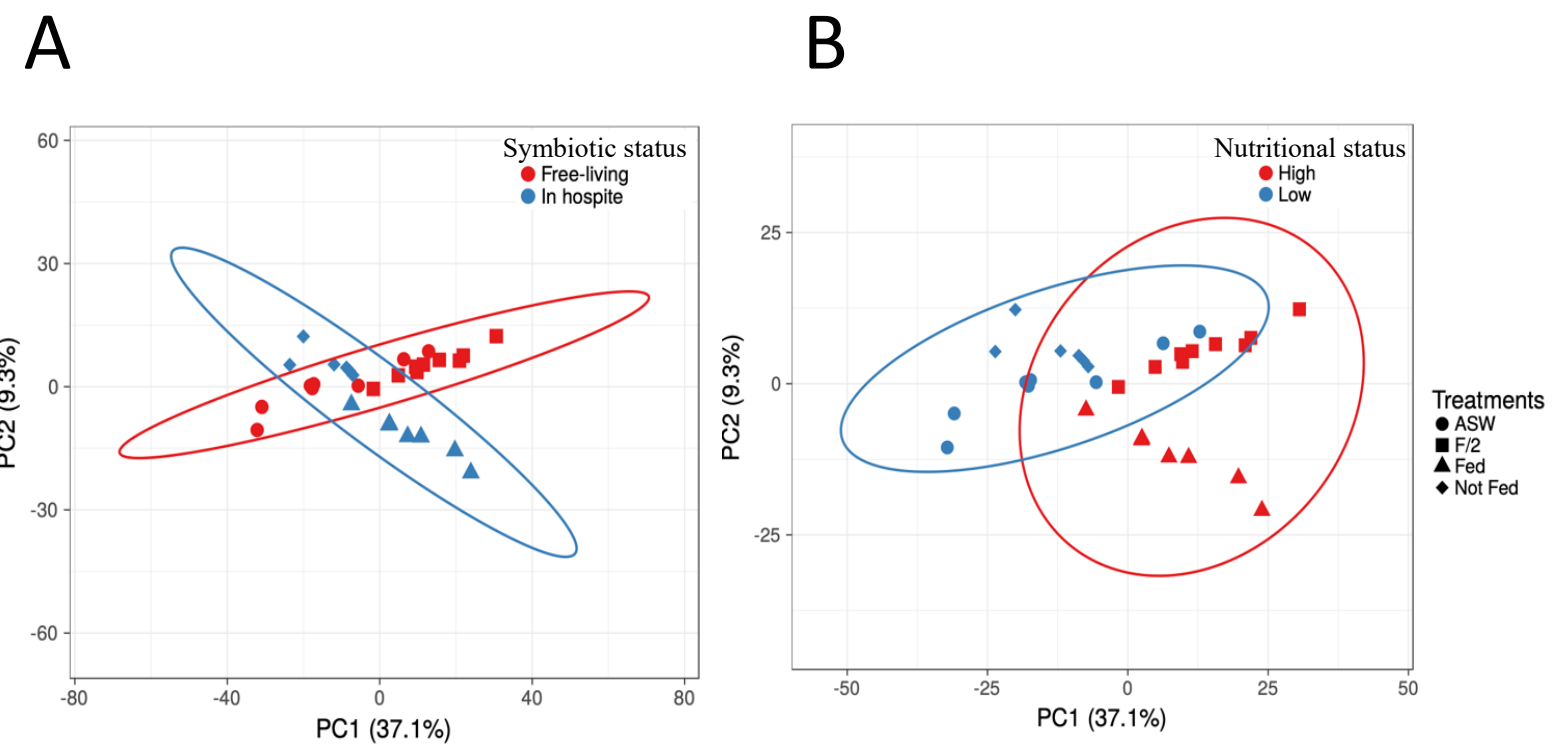

Figure 2.2. Principal component analysis plots of all detected proteins from different treatments of $B$. minutum showing (A) a symbiotic states, when in hospite with the sea anemone Aiptasia and when free-living; and (B) different high (i.e., well-fed and f/2) and low nutritional regimes (i.e., starved and ASW), grouped by ellipses with $99 \%$ confidence intervals.

\subsubsection{Proteomic shifts associated with symbiotic state}

\subsubsection{Requirements for living in a symbiosome}

As previously mentioned, when in hospite, algal cells live inside the symbiosome membrane, which is a late arrested phagosome; and it has been suggested that symbionts avoid host autophagy and immunodetection by manipulation of phosphoinositide (PI) metabolism pathways (Dunn, 2009; Dunn et al., 2007; Wakefield et al., 2000). PI glycerolipids play an important role in signal transduction, cytoskeleton architecture, membrane dynamics and vesicle trafficking routes within eukaryotic cells (Weber et al., 2009). Manipulation of PI metabolism pathways by PI kinases or phosphatases is the main mechanism for cell entry and preventing host autophagy by various microorganisms (Fratti et al., 2001; Hilbi, 2006; Weber et al., 2009). In particular, PI levels on phagosomes are modified by vacuolar pathogens to mimic subcellular compartments or arrest fusion with lysosomes (Weber et al., 2009). Interestingly, a homologue of phosphatidylinositol phosphatase (INP53) was exclusively found in the symbiotic state $\left(q \leq 1.98 \times 10^{-2}\right)$, suggesting its potential function in preventing phagosome maturation. Moreover, a homologue of glycerophosphoinositol permease 1 (GIT1) 
was also only detected in the symbiotic state $\left(q \leq 2.62 \times 10^{-2}\right)$. GIT1 facilitates glycerophosphoinositol (GPI) transport across the cell membrane. GPI is a phospholipid metabolite which is produced by deacylation of PI. GPI has been proposed as a source of phosphate in mildly acidic $\mathrm{pH}$ conditions or in phosphate-limited cells (Almaguer et al., 2004; P. Ziegler et al., 2017). However, it is unknown whether INP53 and GPI work in concert in a functional cnidarian-dinoflagellate symbiosis. In addition, a homologue of Eukaryotic Elongation Factor 1 (eEF1), which is a multifunctional protein without any alternative forms (Sasikumar et al., 2012), was uniquely expressed in symbiosis $(q \leq 2.94 \times$ $\left.10^{-3}\right)$. The canonical function of eEF1 is in translation, however these highly abundant and conserved proteins have also been reported to be involved in immunosuppressive processes by eukaryotic and prokaryotic pathogens (Harvey et al., 2019; Timm et al., 2017; Zhou et al., 2020). However, the immunosuppressive mechanisms of eEF1 in cnidarian-dinoflagellate associations remains to be fully investigated.

Glycanosyltransferases, (1,3- $\beta$-glucanosyltransferases; GAS) are GPI-anchored cell surface proteins that play a role in the elongation of $\beta$-1,3-glucan chains, which have a major role in cell wall assembly, hardening and softening (De Medina-Redondo et al., 2008; Mouyna et al., 2013). The GAS family is essential for cell wall maturation, morphogenesis and virulence (De Medina-Redondo et al., 2008; Mouyna et al., 2005). The dinoflagellate cell wall is typically thinner when in symbiosis to facilitate translocation of nutrients and communication between partners (Colley and Trench, 1983; Schoenberg et al., 1980; Wakefield et al., 2000). Consistent with this observation, two homologues of the GAS family were abundant in the current study, one of which (GAS2) was only found in hospite $\left(q \leq 1.82 \times 10^{-2}\right)$, while the other one (GAS4) was downregulated $\left(q \leq 2.25 \times 10^{-2}\right)$ when in symbiosis, suggesting potential functions of these proteins in symbiont cell wall modification (e.g., softening or hardening) based on physiological needs (Table 2.1).

\subsubsection{Dissolved inorganic carbon transport}

Photosynthesis is a critical feature of the cnidarian-dinoflagellate symbiosis, given the importance of metabolic exchange to this relationship. Symbionts can obtain inorganic carbon for photosynthesis from holobiont respiration (i.e., $\mathrm{CO}_{2}$ ), calcification (in corals, where $\mathrm{CO}_{2}$ is produced as a by-product), or as dissolved inorganic carbon (DIC) in the ambient seawater 
(Davy et al., 2012). However, DIC in ambient seawater is mostly present as bicarbonate $\left[\mathrm{HCO}_{3}{ }^{-}\right]$, and only $\mathrm{CO}_{2}$ can freely pass-through cell membranes.

Table 2-1. Summarized list of proteins that are differentially expressed in Breviolum minutum when comparing in hospite vs. ex hospite, in order of $\log _{2}$ fold-change. Proteins shown are those discussed in the text. The full list of proteins that are differentially expressed in hospite vs. ex hospite is available in Table S2.2 (FDR, q < 0.05).

\begin{tabular}{|c|c|c|c|}
\hline $\begin{array}{l}\text { In vs. ex hospite } \\
\text { fold change }\end{array}$ & $q$-value & Uniprot ID & Uniprot Protein Names \\
\hline Unique, in hospite & $1.98 \mathrm{E}-02$ & A0A1Q9DR34 & Polyphosphatidylinositol phosphatase (INP53) \\
\hline Unique, in hospite & $6.62 \mathrm{E}-03$ & A0A1Q9F6G5 & Nitrilase \\
\hline Unique, in hospite & $2.62 \mathrm{E}-02$ & Q59Q30 & Glycerophosphoinositol permease 1 (GIT1) \\
\hline Unique, in hospite & $2.94 \mathrm{E}-03$ & Q04634 & Elongation factor 1-alpha (eEF1) \\
\hline Unique, in hospite & $1.82 \mathrm{E}-02$ & Q06135 & 1,3-beta-glucanosyltransferase (GAS2) \\
\hline 3.44 & $1.59 \mathrm{E}-06$ & B0Y8K2 & 1,4-beta-D-glucan cellobiohydrolase B (cbhB) \\
\hline 1.78 & $1.33 \mathrm{E}-02$ & A0A1Q9CUD0 & Putative glucose-6-phosphate 1-epimerase \\
\hline 1.76 & $1.14 \mathrm{E}-02$ & Q9SAU2 & Ribulose-5-phosphate-3-epimerase (R5P3E) \\
\hline 1.16 & $9.70 \mathrm{E}-06$ & B4R9R7 & Guanosine monophosphate synthetase (GMP) \\
\hline-0.92 & 8.05E-04 & P11029 & Acetyl-CoA carboxylase (ACC) \\
\hline-1.03 & $2.25 \mathrm{E}-02$ & Q9Y7Y7 & 1,3-beta-glucanosyltransferase (GAS4) \\
\hline-1.10 & 3.34E-04 & Q54WR9 & Type-3 glutamine synthetase \\
\hline-1.49 & $1.73 \mathrm{E}-10$ & Q12613 & Glutamine synthetase (GS) \\
\hline-1.50 & $1.02 \mathrm{E}-05$ & Q00955 & Acetyl-CoA carboxylase (ACC) \\
\hline-1.56 & $1.35 \mathrm{E}-09$ & A0A1Q9CUK2 & GDP-mannose transporter GONST5 \\
\hline-2.04 & $8.68 \mathrm{E}-07$ & Q9LV03 & Glutamate synthase 1 , chloroplastic \\
\hline-3.21 & $1.86 \mathrm{E}-12$ & Q76NM6 & V-type proton ATPase catalytic subunit A \\
\hline-3.90 & 2.49E-09 & A0A1Q9D9X1 & Carbonic anhydrase 2 \\
\hline-3.95 & $3.57 \mathrm{E}-09$ & Q9I262 & Carbonic anhydrase \\
\hline Unique, ex hospite & $6.38 \mathrm{E}-05$ & P74572 & Pyrroline-5-carboxylate reductase (P5CR) \\
\hline Unique, ex hospite & $2.86 \mathrm{E}-02$ & Q9LV03 & Glutamate synthase 1 , chloroplastic \\
\hline Unique, ex hospite & $2.63 \mathrm{E}-05$ & A5CZ28 & Gamma-glutamyl phosphate reductase (GPR) \\
\hline Unique, ex hospite & 4.00E-06 & A0A1Q9CS65 & Carbonic anhydrase 2 \\
\hline Unique, ex hospite & $1.92 \mathrm{E}-10$ & Q9I262 & Carbonic anhydrase \\
\hline Unique, ex hospite & $2.86 \mathrm{E}-02$ & G4N296 & Highly reducing polyketide synthase 19 (PKS19) \\
\hline Unique, ex hospite & $3.76 \mathrm{E}-05$ & P9WQE6 & Phenolphthiocerol/phthiocerol polyketide synthase (PpsA) \\
\hline Unique, ex hospite & $2.72 \mathrm{E}-02$ & P21567 & Alpha-amylase \\
\hline Unique, ex hospite & 9.44E-09 & G2Q9T3 & Polysaccharide monooxygenase \\
\hline
\end{tabular}

Host-derived carbonic anhydrase (CA), which catalyses the conversion of bicarbonate to $\mathrm{CO}_{2}$ and vice versa, is integral to cnidarian carbon concentrating mechanisms (CCMs), as it provides DIC in forms that can be used for both photosynthesis and calcification (Allemand et al., 1998; Furla et al., 2000b). Host CAs increase to support the provision of symbionts with DIC (Oakley et al., 2016; Sproles et al., 2019; Weis, 1991). In the current study, four carbonic 
anhydrase homologues were significantly upregulated ( $>3$-fold) in B. minutum when under either nutrient-limited (ASW) or nutrient-enriched (f/2) conditions, two of which were only found in the free-living state. Moreover, a homologue of V-type ATPase, a proton pump, was upregulated in the free-living state irrespective of nutritional state $\left(3.21\right.$-fold, $\left.q \leq 1.86 \times 10^{-12}\right)$. Proton pumps use ATP as a source of energy to create a proton gradient and function in harmony with carbonic anhydrases, while dinoflagellate genes encoding proton pumps in different symbiotic associations form part of CCMs (Bertucci et al., 2010; Mies et al., 2017). Therefore, upregulated algal CAs and V-Type ATPase in the free-living state might be a response to a lack of readily available $\mathrm{CO}_{2}$ provided by the host CAs and proton pumps.

\subsubsection{Nitrogen metabolism}

Nitrogen flux is an integral part of the cnidarian-dinoflagellate symbiosis, especially in oligotrophic tropical waters (Grover et al., 2008; Miller and Yellowlees, 1989; Rädecker et al., 2017b). Dinoflagellate symbionts assimilate the majority of nitrogen by the holobiont (i.e., whole symbiosis) via the glutamine synthetase/glutamine 2-oxoglutarate amido transferase (GS/GOGAT) pathway, which results in glutamate as the final product, a precursor for other amino acids or purines. The dinoflagellate symbionts can also incorporate ammonium via glutamate dehydrogenase (GDH), which has a lower ammonium affinity. Nevertheless, the cnidarian host also can assimilate nitrogen (Grover et al., 2002; Miller and Yellowlees, 1989; Pernice et al., 2012; Roberts et al., 2001; Summons and Boag, 1986; Wilkerson and Muscatine, 1984; Yellowlees et al., 1994). Eleven different homologues of glutamine synthetase (GS), two homologues of glutamate synthase 1 (GOGAT) and two homologues of glutamate dehydrogenase 2 (GDH) were detected in the current study. However, only four homologues of proteins with predicted functions in the GS/GOGAT pathway were differentially downregulated in hospite in comparison to the free-living state (Table 2.1). This is consistent with previous reports suggesting that this pathway is the major means by which the symbionts assimilate ammonium (Roberts et al., 2001; Wilkerson and Muscatine, 1984). Furthermore, lower expression of GS/GOGAT in the symbiotic state might reflect nitrogen limitation, which may be a primary mechanism by which the host controls the endosymbiont population (Falkowski et al., 1993; Muscatine et al., 1983; Rahav et al., 1989; Wang and Douglas, 1999; Xiang et al., 2020). 
Dinoflagellate symbionts can also exclusively assimilate nitrate and nitrite by converting them to ammonium via specific reductase enzymes (Kopp et al., 2013; Tanaka et al., 2006); upregulation of nitrate and nitrite reductase transcripts has been reported from B. minutum when in hospite (Maor-Landaw et al., 2020). Nitrate transporter and nitrite reductase homologues were detected in the current proteomics dataset, however no differential expression was detected between any of the treatments.

A nitrilase homologue was exclusively detected here in the symbiotic state under both nutrient regimes. Nitrilases hydrolyse nitrile compounds to carboxylic acid and ammonia, and are found in a broad range of organisms, including bacteria, fungi and plants. Nitrilases are also involved in defence, detoxification and plant hormone synthesis (Howden and Preston, 2009). Nitrilase has been reported to be downregulated during nitrogen starvation in marine green algae (Lauritano et al., 2019). In the current study, a nitrilase homologue was particularly abundant in the symbionts of both starved and heavily-fed anemones $\left(q \leq 6.62 \times 10^{-3}\right)$ rather than in the free-living state, and it was most abundant in well-fed anemones. This may indicate that the symbionts respond to nitrogen starvation inside the host by hydrolysing nitrile compounds for ammonia.

With respect to nitrogen fate after assimilation, a group of 23 proteins were detected with predicted functions in methionine de novo synthesis and metabolism, of which 10 were upregulated in one or more of the different treatments (Table S2.4). Methionine is required for protein synthesis and has been previously reported to be important in the cnidariandinoflagellate symbiosis, with both cnidarian host and dinoflagellate symbiont possessing methionine metabolism pathway enzymes (Shinzato et al., 2014). Moreover, the native $B$. minutum has been hypothesized to support the host with greater amounts of methionine in comparison to non-native Durusdinium trenchii (Sproles et al., 2019). Unsurprisingly, given the links to protein synthesis, methionine pathway proteins in the current study were upregulated more in both the nutrient-enriched $(\mathrm{f} / 2)$ culture and well-fed anemone treatments than in the low nutrient/starved treatments, with $\mathrm{f} / 2$-grown algae having the greatest expression of these proteins. 
Two important enzymes in proline biosynthesis from glutamate, gamma-glutamyl phosphate reductase (GPR) and pyrroline-5-carboxylate reductase (P5CR) (Nandakumar et al., 1999; Szabados and Savouré, 2010), were only detectable in the free-living state irrespective of nutritional state. Proline biosynthesis and accumulation typically increase under different environmental stresses such as high salinity, UV radiation, heavy metals, oxidative stress and biotic stress (Szabados and Savouré, 2010). Symbionts living inside the symbiosome experience ionic conditions similar to their host, which has lower sodium and calcium concentrations than ambient seawater (Goiran et al., 1997). Moreover, they are also selfshaded, with the local irradiance depending on tentacle contraction and their location within the host (Glider et al., 1980). Increased proline biosynthesis might therefore be indicative of a response to more stressful conditions when living outside of their natural habitat. The other way for incorporating glutamine by Symbiodiniaceae is via the purine pathway, which facilitates the storage of high-nitrogen compounds (Pernice et al., 2012). A homologue of guanosine monophosphate synthetase (GMP), which functions in de novo synthesis of guanine nucleotides by hydrolysing glutamine (Nakamura et al., 1995), was significantly more expressed in the free-living state $\left(1.16\right.$-fold, $\left.q \leq 9.7 \times 10^{-6}\right)$, and was especially abundant in the $\mathrm{f} / 2$ culture treatment.

\subsubsection{Carbohydrate and fatty acid metabolism}

In total, a group of 20 proteins that are involved in carbohydrate metabolism were differentially expressed, 17 of which were exclusively detected in the free-living state irrespective of nutrient status (Table S2.5). In contrast, homologues of ribulose-5-phosphate-3-epimerase and glucose6-phosphate 1-epimerase, both of which are components of the rate-limiting part of the pentose phosphate pathway (PPP), were upregulated in symbiosis. The pentose phosphate pathway, being parallel to glycolysis, is fundamental to maintaining cellular carbon homoeostasis, biosynthesis of nucleotides and amino acids, moderating oxidative stress, and anabolism of reducing molecules (Kruger and Von Schaewen, 2003; Stincone et al., 2015).

Seven proteins of the aforementioned group were extracellulases, such as alpha-amylase, polysaccharide monooxygenase and 1,4-beta-D-glucan cellobiohydrolase B (cbhB), that hydrolyse large polysaccharides to smaller sugars like glucose (Table S2.5) (Huang et al., 1992; Labourel et al., 2020; Nutt et al., 1998). Interestingly, a homologue of extracellular cbhB 
was upregulated most in symbiosis, though a larger number of extracellular cellulose and carbohydrate binding proteins were more abundant in the free-living state. The function of these extracellulases is unknown, but they could relate to the cell wall, which in the Symbiodiniaceae is composed primarily of cellulose and glycoproteins (Markell et al., 1992). In particular, the discarding of cellulosic thecal plates during ecdysis, where the plasma membrane and thecal vesicles are shed and new thecal vesicles are formed, is well known in these algae, with shed thecal plates being reported to accumulate at the bottom of culture flasks or inside the symbiosome membrane (Trench and Blank, 1987; Wakefield et al., 2000; Wakefield and Kempf, 2001). It may therefore be that these proteins play a role in digesting shed cellulosic thecal plates, whether inside the symbiosome or while free-living. The fate of digested polysaccharide complexes in the symbiosome remains to be elucidated, but it is noteworthy that an increased abundance of the algal sugar transporter SWEET1 was also observed in ASW culture treatment (Table S2.1, $q<1.59 \times 10^{-2}$ ), which might indicate that free-living algae scavenge on shed thecal plates or dead cells.

Lipids and fatty acids are the major form of energy storage in dinoflagellate symbionts, and reflect the nutritional state of the holobiont (Chen et al., 2012; Wang et al., 2013). Two homologues of acetyl-CoA carboxylase, which is the key enzyme in the fatty acid synthetic pathway, along with a group of other plastid-localised or cytosolic proteins involved in this pathway, were detected in all treatments. However, this group was significantly more abundant in the free-living state (Table S2.6). Different members of Symbiodiniaceae have different strategies for energy and fatty acid storage which can be modified by the host (Matthews et al., 2018, 2017; Rosset et al., 2019; Wang et al., 2015). Moreover, lower contents of lipid and fatty acids have been detected in cultured Symbiodiniaceae in comparison to when in hospite (Wang et al., 2015). It is unclear why proteins involved in lipid and fatty acid pathways are upregulated when ex hospite in the current study, though it could be due to release from host metabolic control when ex hospite.

Furthermore, four different homologues of polyketide synthase, such as phenolphthiocerol/phthiocerol polyketide synthase (PpsA) and highly reducing polyketide synthase 19 (PKS19), were exclusively found in free-living symbionts under both nutritional regimes. Polyketides, formed from acyl building blocks, are the backbone of some biological 
toxins. Polyketide metabolites in Symbiodinium sp., as well as polyketide synthetase genes from B. minutum, have been reported before (Beedessee et al., 2015; Nakamura et al., 1998; Verma et al., 2019). PpsAs have diverse biological functions, including having antifungal and antibacterial properties, however these proteins are also involved in infection or increasing immunity in other symbiotic associations (Mukherjee et al., 2012; Navarro-Muñoz and Collemare, 2020; Waddell et al., 2005; Yang et al., 1996). Therefore, it is not clear if algal polyketide synthase is involved in invasion of the cnidarian host by the symbiont or whether it is an antibiotic defence mechanism against other microorganisms, such as those commonly associated with Symbiodiniaceae cultures (Xiang et al., 2013).

\subsubsection{Proteomic shifts associated with nutritional state}

\subsubsection{Photosynthesis}

Photosynthesis is central to the cnidarian-dinoflagellate symbiosis (described in detail in Davy et al., 2012). A large number of proteins that have predicted functions in photosynthesis were identified from all the treatments in the current study. However, a group of photosystem (PS) I and II proteins involved in light harvesting, including fucoxanthin-chlorophyll $a-c$ binding (FCBs), chlorophyll $a-b$ binding (LHC) and caroteno-chlorophyll $a$-c-binding proteins, as well as chloroplastic electron transport chain-like cytochromes, ferredoxin-NADP reductases, P700, PS I and II reaction centres, and D1 and D2 proteins were highly upregulated in the nutrientenriched treatments (Table S2.7). Electron transport in chloroplasts is fuelled by electrochemical energy from PS II and PS I, during which ATP and reductant NADPH are generated and $\mathrm{O}_{2}$ evolves (Warner and Suggett, 2016). In conjunction with this, proteins that function in different stages of Calvin cycle, such as ribulose-1,5-bisphosphate carboxylase/oxygenase (RuBisCO), fructose-1,6-bisphosphatase 1 (FBPase1), glyceraldehyde-3-phosphate dehydrogenase and phosphoribulokinase, were also most abundant in the high nutrient treatments (Table S2.7). Fixation of carbon dioxide to sugar phosphates happens in the Calvin cycle by using ATP and NADPH generated from light harvesting (Nickelsen, 2015). It has been shown in past studies that the amount of chlorophyll per symbiont cell, photosynthetic efficiency and capacity, and the Calvin cycle rate all increase in a high nutrient environment (Dubinsky et al., 1990; Karako-Lampert et al., 2005; Roberty et al., 2020). Conversely, low nutrient availability induces photoinhibition, reduced chlorophyll content per cell, reduced quantum yield $\left(\mathrm{F}_{\mathrm{v}} / \mathrm{F}_{\mathrm{m}}\right)$, and non-photochemical 
quenching (Borell and Bischof, 2008; Dubinsky et al., 1990; Ferrier-Pagès et al., 2000; Fitt and Cook, 2001; Hoogenboom et al., 2012), as was also seen in the current study (Fig. 2.1B). Therefore, the upregulation of PS and Calvin cycle proteins seen here is very likely simply due to the increased availability of nutrients for protein synthesis by the dinoflagellate symbionts.

\subsubsection{Oxidative stress under high nutrient regimes}

Photosynthesis and respiration inevitably create reactive oxygen species (ROS) as by-products which can further damage DNA, proteins and cellular membranes (Venn et al., 2008; Wietheger et al., 2018). The importance of Symbiodiniaceae ROS generation and antioxidant activity in response to stressful conditions, such as increased temperature or irradiance, has been investigated before (Krueger et al., 2014; McGinty et al., 2012; Rehman et al., 2016; Roberty et al., 2016; Wietheger et al., 2018). Superoxide $\left(\mathrm{O}_{2}^{-}\right)$is produced by PSs I and II, however it is reduced to hydrogen peroxide $\left(\mathrm{H}_{2} \mathrm{O}_{2}\right)$ by superoxide dismutase (SOD) and further to $\mathrm{H}_{2} \mathrm{O}$ by ascorbate peroxidase (APX) or catalase (Wietheger et al., 2018). Homologues of symbiont antioxidants, such as APX and SOD, were more abundant in the two high nutrient treatments (i.e., f/2 and well-fed). Moreover, four homologues of glutathione s-transferase and a homologue of chloroplastic glutathione reductase, all of which are important in the oxidative stress response and cell signalling, were more abundant in the high nutrient treatments. Increased gross photosynthesis in Symbiodiniaceae under nutrient enrichment has been reported in the past (Ferrier-Pagès et al., 2000). Thus, the increased expression of several symbiont antioxidant mechanisms in response to nutrient enrichment might be a cellular response for maintaining high rates of photosynthesis/respiration (Davy et al., 2006; Foyer and Shigeoka, 2011) (Table 2.2).

A homologue of metacaspase-1B (casB) was also upregulated in the well-fed treatment (2.35fold, $q<1.35 \times 10^{-2}$ ), possibly due to stress induced by the high symbiont population density in the host's tissues (Dunn et al., 2004; Segovia, 2008). Oxidative stress can cause programmed cell death (apoptosis) in Symbiodiniaceae (Franklin et al., 2004; Richier et al., 2006). One of the key groups of proteins involved in oxidative stress-induced apoptosis, from Protozoa to plants, are the metacaspases, however these proteins also function in cell division (Tsiatsiani et al., 2011; Watanabe and Lam, 2011). Metacaspases, being ancestors of metazoan caspases, are cysteine-type proteases (Watanabe and Lam, 2011). Caspases from cnidarian- 
dinoflagellate associations function in symbiont expulsion from the host cell during stress (Dunn et al., 2007; Hawkins et al., 2013). More recently, metacaspase transcripts have been shown to be upregulated by Symbiodiniaceae when in hospite as a response to thermal and $\mathrm{pH}$ stress (Gierz et al., 2017; Lin et al., 2018). Nevertheless, whether casB activity is essential for cell cycle progression or is a response to oxidative stress-induced apoptosis remains to be clearly demonstrated.

Table 2-2. Summarized list of proteins that are differentially expressed in Breviolum minutum when comparing high nutrient $v s$. low nutrient regimes (i.e. $\mathrm{f} / 2$ and well-fed treatments $v s$. ASW and starved treatments), in order of $\log _{2}$ fold-change. Proteins shown here are those discussed in the text. The full list of proteins that were differentially expressed between high $v s$. low nutrient regimes is available in Table S2.3 (FDR, q $<0.05$ ).

\begin{tabular}{|c|c|c|c|}
\hline $\begin{array}{l}\text { High vs Low nutrient } \\
\text { regime fold change }\end{array}$ & $q$-value & Uniprot ID & Uniprot Protein Names \\
\hline Unique, High & $2.12 \mathrm{E}-09$ & A0A1Q9CJD4 & Glutathione S-transferase \\
\hline Unique, High & $1.26 \mathrm{E}-06$ & A0A1Q9CL40 & Multicopper oxidase (MCO) \\
\hline 3.54 & $9.74 \mathrm{E}-28$ & O09452 & Glyceraldehyde-3-phosphate dehydrogenase \\
\hline 3.01 & $1.49 \mathrm{E}-16$ & P26302 & Phosphoribulokinase (PRK) \\
\hline 2.88 & $1.80 \mathrm{E}-21$ & P00110 & Cytochrome $c 6$ (Cytochrome c-553) \\
\hline 2.87 & $8.12 \mathrm{E}-24$ & Q40296 & Fucoxanthin-chlorophyll $a-c$ binding protein $\mathrm{B}$, chloroplastic \\
\hline 2.84 & $8.15 \mathrm{E}-05$ & P00110 & Cytochrome $c 6$ (Cytochrome c-553) \\
\hline 2.78 & $7.11 \mathrm{E}-20$ & Q40297 & Fucoxanthin-chlorophyll $a-c$ binding protein A, chloroplastic \\
\hline 2.77 & $9.74 \mathrm{E}-28$ & Q95AG0 & Cytochrome f \\
\hline 2.76 & $9.31 \mathrm{E}-25$ & A0T0C6 & Cytochrome $c$-550 (Cytochrome c550) \\
\hline 2.68 & $3.29 \mathrm{E}-15$ & Q9XQV2 & Photosystem I P700 chlorophyll a apoprotein A2 \\
\hline 2.58 & $2.78 \mathrm{E}-18$ & Q85FP8 & Photosystem I reaction centre subunit XI \\
\hline 2.53 & $6.16 \mathrm{E}-13$ & Q9SDM1 & Chlorophyll $a-b$ binding protein \\
\hline 2.53 & $7.49 \mathrm{E}-13$ & Q00598 & Ferredoxin--NADP reductase \\
\hline 2.5 & $1.28 \mathrm{E}-13$ & P55738 & Caroteno-chlorophyll $a$-c-binding protein \\
\hline 2.39 & $7.38 \mathrm{E}-29$ & Q41406 & Ribulose bisphosphate carboxylase (RuBisCO) \\
\hline 2.35 & $1.35 \mathrm{E}-02$ & A0A1Q9EQB8 & Metacaspase-1B \\
\hline 2.34 & $6.54 \mathrm{E}-21$ & P49472 & Photosystem II CP43 reaction centre protein \\
\hline 2.32 & $5.74 \mathrm{E}-08$ & P25851 & Fructose-1,6-bisphosphatase 1, (FBPase1) (ELECTRON FLOW 1) \\
\hline 2.31 & $9.22 \mathrm{E}-17$ & P54375 & Superoxide dismutase (SOD) \\
\hline 2.23 & $2.48 \mathrm{E}-19$ & A0T0T0 & Photosystem II D2 protein \\
\hline 1.69 & $2.34 \mathrm{E}-12$ & Q9MSC2 & Photosystem II protein D1 \\
\hline 1.64 & $4.16 \mathrm{E}-02$ & P20136 & Glutathione S-transferase 2 (GST-CL2) \\
\hline 1.47 & $5.08 \mathrm{E}-07$ & Q7XJ02 & Probable L-ascorbate peroxidase 7 (APX) \\
\hline 1.39 & $1.22 \mathrm{E}-08$ & P46434 & Glutathione S-transferase 1 \\
\hline 1.21 & $2.67 \mathrm{E}-02$ & P42770 & Glutathione reductase, chloroplastic (GR) \\
\hline 0.92 & $2.37 \mathrm{E}-02$ & P46429 & Glutathione S-transferase 2 \\
\hline-1.07 & $1.65 \mathrm{E}-04$ & P42577 & Soma ferritin \\
\hline
\end{tabular}




\subsubsection{Iron metabolism under different nutrition regimes}

Iron is essential for photosynthetic microalgae, as it is needed for many cellular processes and biochemical pathways, such as DNA synthesis, respiration and photosynthesis (Raven et al., 1999; Sunda, 2012). In particular, iron cofactors are crucial in photosynthetic electron transport (Kroh and Pilon, 2020). In Symbiodiniaceae, iron is the key trace metal in cell growth (Rodriguez et al., 2016; Rodriguez and Ho, 2018). Moreover, iron uptake in Symbiodiniaceae (specifically B. minutum) increases at elevated iron concentrations, while iron utilization increases in efficiency in iron-depleted environments (Reich et al., 2020). This is consistent with the findings from the current study, in which multicopper oxidase (MCO) was only detected under nutrient enrichment (i.e., $\mathrm{f} / 2$ and well-fed anemones). MCOs are involved in the iron uptake pathway by oxidizing iron II to iron III in unicellular algae (Groussman et al., 2015; Herbik et al., 2002). Interestingly, in another study, coral symbionts were identified as the main destination for heterotrophically-acquired iron (Ferrier-Pagès et al., 2018). This might help to explain the even higher expression levels of MCOs in symbionts from heavily-fed anemones $v s$. their free-living counterparts in $\mathrm{f} / 2$ medium. In contrast, algae in the low nutrient regime (ASW and starved combined) increased expression of a homologue of soma ferritin (1.07-fold, $\left.q \leq 1.65 \times 10^{-4}\right)$, an important iron storage protein. Economizing iron storage via ferritin in marine unicellular algae has been suggested as a common strategy for living in irondepleted environments (Arosio et al., 2017).

\subsection{Conclusion}

The current research identified proteins in B. minutum that are differentially expressed between the in and ex hospite states. The predicted functions of these proteins relate to the establishment and maintenance of a functional cnidarian-dinoflagellate symbiosis. Homologues of inositol permease and phosphatase, along with other immunosuppressant proteins were only expressed in hospite, indicating active manipulation of host cellular immune mechanisms by the native symbiont, possibly to arrest phagosome maturation. Additionally, homologues of carbonic anhydrases were expressed only when ex hospite emphasizing the importance of DIC for algal growth and maintenance. Proteins with predicted functions in nitrogen assimilation were relatively less abundant in hospite; and a homologue of nitrilase with a predicted function in ammonium uptake from nitrile compounds was expressed exclusively in hospite. All of these observations are consistent with previous reports showing nitrogen limitation of the symbionts 
by the host (Wang and Douglas, 1999, 1998; Xiang et al., 2020). Nutrient enrichment had a direct impact on PS, Calvin cycle and iron metabolism proteins. In addition, increased photosynthesis under nutrient enrichment was accompanied by oxidative stress and apoptosis. These data provide new target proteins and pathways in the symbionts for further study of the cnidarian-dinoflagellate symbiosis. 


\section{Chapter 3 \\ The influence of symbiosis on the proteome of a native and non-native symbiont during colonisation of the model cnidarian Aiptasia.}

\subsection{Introduction}

Coral reefs are among the most diverse and productive tropical ecosystems. Hermatypic corals are fundamental to these ecosystems and provide a broad range of ecological services, such as providing habitat for other reef organisms (Hoegh-Guldberg, 1999; Kleypas et al., 2021). The success of corals is dependent on their obligatory endosymbiotic relationship with photosynthetic dinoflagellate algae from the family Symbiodiniaceae (Veron et al., 2009). Given the oligotrophic nature of tropical waters, the metabolic exchange between the symbiotic partners has profound importance, with the symbionts translocating photosynthate to the host and in return the host providing them with inorganic carbon, nitrogen and phosphorus, as well as shelter (Muscatine and Porter, 1977). The main form in which photosynthetically-fixed carbon is translocated to the host is glucose (Burriesci et al., 2012; Hillyer et al., 2017b; Suescún-Bolívar et al., 2016). Nevertheless, other forms of photosynthate are also translocated to the host, including essential amino acids, fatty acids, lipids, triacylglycerol and sterols (Dunn et al., 2012; Hillyer et al., 2016; Wang and Douglas, 1999; Yamashiro et al., 1999).

The family Symbiodiniaceae contains eleven described genera to date, which are genetically, physiologically and ecologically diverse (LaJeunesse et al., 2018; Nitschke et al., 2020; Pochon and LaJeunesse, 2021). Moreover, they have different host specificities, with some members being host-generalists, having the ability to form symbioses with a wide range of hosts, while some cnidarian hosts can only harbour a single symbiont species (Berkelmans and Van Oppen, 2006; Rowan et al., 1997; Santos et al., 2004; Xiang et al., 2013). The basis of host-symbiont specificity has not yet been fully described, however metabolic exchange and cellular recognition between partners are important influencing factors (Bay et al., 2011; Davy et al., 2012; Matthews et al., 2017). Interactions between cnidarian and Symbiodiniaceae partners exhibit a very wide range of diversity, from mutualism to parasitism, with some 
symbionts being less beneficial to their host, for example by having lower rates of photosynthate translocation, or disrupting host metabolism via competing for important nutrients such as nitrogen (Baker et al., 2018; Jones and Berkelmans, 2011; Lesser et al., 2013; Stat et al., 2008).

Despite efficient metabolic integration, the cnidarian-dinoflagellate symbiosis is susceptible to environmental change and stress. For example, under thermal stress, the dinoflagellate symbionts become less beneficial and are ultimately lost from the host's tissues, in a process commonly known as 'coral bleaching' (Hoegh-Guldberg et al., 2007; Hughes et al., 2017). Nevertheless, some species of Symbiodiniaceae are more resistant to temperature anomalies, so conferring more thermal tolerance to the holobiont, i.e. the overall symbiosis (Hume et al., 2015; LaJeunesse et al., 2014). For example, Durusdinium trenchii, a host generalist, has been found to be the dominant symbiont after bleaching events, though less beneficial for the host; cnidarian hosts harbouring D. trenchii exhibit slower rates of growth, and calcification, and elevated oxidative stress and immune responses (Kennedy et al., 2015; Little et al., 2004; Pettay et al., 2015; Sproles et al., 2019).

Symbiont identity is therefore crucial for determining the fate of the holobiont and hence the overall reef ecosystem, with the proposal that corals may be able to adapt to the changing environment by either 'symbiont switching' (i.e., acquiring symbionts de novo from the environment), or 'symbiont shuffling' (i.e., changing the relative abundance of the existing symbiont communities within colonies). In particular, these mechanisms could favour symbionts that are better suited to the prevailing thermal conditions, thereby reducing susceptibility to bleaching (Baker, 2001; Berkelmans and Van Oppen, 2006; Buddemeier and Fautin, 1993; Cunning et al., 2015b; Rowan et al., 1997; van Oppen et al., 2009).

Understanding the processes involved in symbiosis establishment and dysfunction, as well as the cellular mechanisms that determine patterns of host-symbiont specificity, is therefore important, though there are still major gaps in our knowledge (Davy et al., 2012). Moreover, the majority of cellular studies have been focused on the cnidarian host rather than the symbiont (Bay and Palumbi, 2015; Lehnert et al., 2014, 2012; Oakley et al., 2017, 2016; Sproles et al., 
2019), so limiting our understanding of the bi-directional fluxes and signalling that underlie the success of the cnidarian-dinoflagellate symbiosis. Exaiptasia pallida, commonly known as 'Aiptasia', is a widely adopted model organism for the cnidarian-dinoflagellate symbiosis (Baumgarten et al., 2015; Matthews et al., 2017; Weis et al., 2008). Across the Indo-Pacific region, Aiptasia naturally harbours B. minutum, however other genera of symbionts are also able to partner it. For example, Florida populations of this anemone predominantly harbour species of Symbiodinium and Breviolum, as well as Cladocopium (Thornhill et al., 2013). However, under experimental conditions, Aiptasia can establish a symbiosis with numerous different symbiont species, though non-native symbionts typically function less optimally, causing the symbiosis to be less beneficial to the host (Gabay et al., 2019; Matthews et al., 2018, 2017; Oakley et al., 2017, 2016; Sproles et al., 2019). By comparison, how the symbiont responds to colonisation of a non-native host is not known, though there have been a limited number transcriptomic, proteomic and lipidomic studies of Symbiodiniaceae when in symbiosis with their native host $v s$. free-living. These studies have identified differences in photosynthesis, transmembrane transport, and cell division and signalling between these two symbiotic states, though we do not yet know what happens during the earlier stages of host colonisation (Bellantuono et al., 2019; Chen et al., 2015; Maor-Landaw et al., 2020; Pasaribu et al., 2015; Weston et al., 2012). Elucidating the symbiont-specific responses that underlie symbiosis establishment and host-symbiont specificity is important, and will complement what we know about the host response. Ultimately, it will aid our understanding of the cellular foundations of a successful symbiosis and the potential for corals to adapt to climate change through changing their symbiotic partners.

I therefore aimed to compare and contrast the proteome of native B. minutum and non-native D. trenchii when in symbiosis with Aiptasia. The proteome of B. minutum was characterised across multiple time-points during colonisation and then compared to the proteome of $D$. trenchii in the fully-established symbiotic state. I hypothesised that colonisation would be accompanied by an upregulation of proteins associated with symbiosis establishment and cell proliferation, plus inter-partner signalling and metabolic exchange, but that these changes would be less pronounced in the non-native symbiont and might even be accompanied by signs of cellular stress. 


\subsection{Materials and Methods}

\subsubsection{Experimental organisms}

Two Symbiodiniaceae species - the native B. minutum and non-native D. trenchii - were grown for eight weeks prior to experimentation in Guillard's f/2 medium in $0.22 \mu \mathrm{m}$-filtered seawater (FSW), at $25^{\circ} \mathrm{C}$ and $100 \mu \mathrm{mol}$ photons $\mathrm{m}^{-2} \mathrm{~s}^{-1}$ irradiance on a $12 \mathrm{~h}: 12 \mathrm{~h}$ light:dark cycle. $B$. minutum (culture ID: FLAp2) was originally isolated from Aiptasia, and D. trenchii (culture ID: Ap2) was isolated from an unknown anemone in Okinawa (Japan). To confirm the identity of these cultures, DNA was extracted using CTAB/phenol-chloroform (Baker et al., 2004), and the internal transcribed spacer (ITS2) region of ribosomal DNA amplified by polymerase chain reaction using ITSintfor2 and ITS2Rev2 as the forward and reverse primers, respectively (LaJeunesse and Thornhill, 2011; Thornhill et al., 2007). Amplicons were directly sequenced (Macrogen Inc., Seoul, South Korea), and sequences aligned with Geneious Prime v. 2019.2.3 (Biomatters Ltd., Auckland, NZ). A custom BLAST search was carried out against Symbiodiniaceae ITS2 sequences in Geosymbio (Franklin et al., 2012).

Specimens of Aiptasia $(\mathrm{n} \sim 200)$ were taken from a clonal laboratory stock (strain ID: NZ1; originally from the Indo-Pacific region) and rendered aposymbiotic (i.e. symbiont-free) by incubating them in $0.19 \mathrm{mM}$ menthol with $5 \mu \mathrm{M}$ 3-(3,4-dichlorophenyl)-1,1-dimethylurea (DCMU) as the photosynthetic inhibitor for four weeks (Matthews et al., 2016). The absence of symbionts was confirmed via a lack of chlorophyll autofluorescence, assessed by confocal microscopy (100× magnification, Olympus Provis AX70). All anemones were fed twice per week with Artemia sp. nauplii and maintained in the dark at $25^{\circ} \mathrm{C}$ prior to the experiment.

\subsubsection{Inoculation of Aiptasia with symbiotic algae}

Aposymbiotic anemones $(\mathrm{n} \sim 400)$ were starved for one week prior to inoculation with algal cultures in $250 \mathrm{ml}$ containers (20 anemones per container). One drop of symbiont cell suspension, concentrated by centrifugation to $\sim 3 \times 10^{6}$ cells $\mathrm{mL}^{-1}$, mixed with a dilute suspension of Artemia sp. nauplii to induce phagocytosis, was pipetted onto each anemone's oral disc ( $\mathrm{n} \sim 200$ anemones per symbiont species). Anemones were incubated in this symbiont suspension for $24 \mathrm{~h}$ before the FSW was changed. All anemones were fed with Artemia sp. nauplii three times per week and maintained for 14 weeks at $25{ }^{\circ} \mathrm{C}$ under $100 \mu$ mol photons 
$\mathrm{m}^{-2} \mathrm{~s}^{-1}$ irradiance on a $12 \mathrm{~h}: 12 \mathrm{~h}$ light:dark cycle. Anemones were sampled at Weeks 4, 8 and 14 by pooling $\mathrm{n} \sim 5$ anemones from each container separately and rapidly freezing in liquid nitrogen. Anemones were then homogenized using a $5 \mathrm{ml}$ glass tissue homogenizer at $4{ }^{\circ} \mathrm{C}$ in $2 \mathrm{ml} \mathrm{FSW}$, and symbionts were separated from the host by centrifugation at $500 \mathrm{xg}$ for $5 \mathrm{~min}$ at $4{ }^{\circ} \mathrm{C}$. The host fraction (i.e., supernatant) was used for total protein quantification with a Qubit® 2.0 Fluorometer and Protein Assay Kit (ThermoFisher Scientific). The symbiont pellet was washed at $4{ }^{\circ} \mathrm{C}$ with $500 \mu \mathrm{FSW}, 10 \mu \mathrm{l}$ were aliquoted for cell counts, and finally the remaining $490 \mu \mathrm{l}$ were pelleted again by centrifugation at $500 \mathrm{x}$ g for $5 \mathrm{~min}$ at $4{ }^{\circ} \mathrm{C}$. Free-living algal cultures were sampled before inoculation of the hosts and immediately flash-frozen in liquid nitrogen (i.e., Week 0). All samples were kept at $-80^{\circ} \mathrm{C}$ until downstream analysis.

\subsubsection{Protein extraction}

Algal pellets were washed with $500 \mu \mathrm{l}$ cold HPLC-grade water (Sigma-Aldrich) at $4{ }^{\circ} \mathrm{C}$ to remove salts, and resuspended in $500 \mu 1$ 1\% sodium dodecyl sulphate (SDC) in HPLC-grade water. The algal suspension was passed five times through a 23 -gauge needle $(0.337 \mathrm{~mm}$ inner diameter) to dislodge any remaining host cell debris (e.g., symbiosome membrane) or protein of host origin from the algal cells. All the samples were centrifuged again at $500 \mathrm{x}$ g for $5 \mathrm{~min}$ at $4{ }^{\circ} \mathrm{C}$ and the host-containing supernatant discarded. For consistency, this process was repeated for Week 0 algae. The algal pellet was resuspended in $500 \mu 15 \%$ SDC in HPLC-grade water, and the cells disrupted with an ultrasonic homogenizer (Vibra-Cell ${ }^{\mathrm{TM}}$ Ultrasonic VCX 500). Total protein extraction was conducted by incubating homogenized cells at $85{ }^{\circ} \mathrm{C}$ for 20 min with 1\% B-mercaptoethanol (BME). Residual hydrophobic pigments were removed by ethyl acetate phase transfer (Yeung and Stanley, 2010). Filter aided sample preparation (FASP) was used for removal of SDC and overnight protein digestion was conducted with $2 \mu \mathrm{g}$ trypsin at $37{ }^{\circ} \mathrm{C}$. Digested peptides were centrifuged into a new $0.5 \mathrm{ml}$ Protein LoBind ${ }^{\circledR}$ tube (Eppendorf). Formic acid (final concentration 1\%) was used to terminate trypsin and precipitate any remaining SDC. Peptides were then desalted using 50\% and 30\% acetonitrile (ACN) and resuspended in $0.1 \%$ formic acid at $37{ }^{\circ} \mathrm{C}$ for $30 \mathrm{~min}$, as described in Oakley et al. (2016). Symbiont cell densities in anemones hosting D. trenchii at Weeks 4 and 8 were insufficient to provide enough protein for LC-ESI-MS/MS, so only samples for Week 0 (i.e., prior to inoculation) and Week 14 were processed for this species. 


\subsubsection{LC-ESI-MS/MS}

Liquid chromatography-electrospray ionization-tandem mass spectrometry (LC-ESI-MS/MS) was used for peptide analysis. $200 \mathrm{ng}$ of peptides per sample were separated by liquid chromatography (Ultimate 3000, Dionex) on a PepMap C18 column (\#160321, Thermo Scientific) at $35{ }^{\circ} \mathrm{C}$, with a non-linear gradient from $96 \%$ to $50 \%$ buffer A $(0.1 \%$ formic acid $)$ to buffer B ( $80 \%$ acetonitrile, $0.1 \%$ formic acid) and a flow rate of $0.3 \mu$ for $90 \mathrm{~min}$. An Orbitrap Fusion Lumos Tribrid mass spectrometer (Thermo Scientific) was used to analyse hyphenated peptides, which were ionized by an electrospray needle at $1.8 \mathrm{kV}$. An orbitrap with a resolution of $120 \mathrm{k}$ and scan range of 375-1599 (m/z) was used for acquiring MS spectra. Sequencing was performed via higher energy collisional dissociation (HCD) fragmentation on the top 20 precursors, and fragment ion spectra (MS/MS) were then acquired in the linear ion trap with dynamic exclusion enabled for $60 \mathrm{~s}$. Thermo Xcalibur (v4.3) was used to control LCESI-MS/MS instruments.

\subsubsection{Protein identification and quantification}

The Andromeda algorithm in MaxQuant v1.6.12.0 (Cox et al., 2014) was used to search the resulting tandem MS spectra separately against a custom protein database constructed from $B$. minutum and D. trenchii transcriptomes (Parkinson et al., 2016), with standard genetic code for translation. A built-in contaminants database from MaxQuant was also coupled in the search. A maximum of two missed cleavages of trypsin digestion, a minimum of two matching peptides per protein, and a minimum peptide length of seven amino acids, all with a false discovery rate of 0.01 , were required for peptide and protein validations. Variable modifications were set as N-terminus carbamylation and oxidation of methionine, and fixed modifications were specified as cysteine carbamidomethylation. All spectra from in hospite treatments were also searched against Aiptasia protein sequence databases (Baumgarten et al., 2015 ) to confirm that host contamination was minimal.

\subsubsection{Statistical analyses}

Cell count data were checked for normality by the Shapiro-Wilk test. Homogeneity of variance and covariances were checked by Levene's test and Box's M-test, respectively. A mixed twoway analysis of variance (ANOVA) was used to test for differences $(p<0.05)$ in symbiont 
density between symbiont species and days, followed by a Bonferroni post hoc test to identify specific pairwise differences. All physiological data analyses were conducted in $\mathrm{R}$ 4.0.3 ( $\mathrm{R}$ Core Team, 2020).

Bioinformatics software Perseus v.1.6.13.0 (Tyanova and Cox, 2018) was used to remove known contaminant proteins and false identifications. The remaining protein precursor intensity was $\log _{2}$-normalized and further used to compare and identify significantly different proteins between treatments. Principal component analysis (PCA) plots were generated with ClustVis (Metsalu and Vilo, 2015). A recently described robust statistical test method, PolyStest integrated with Miss Test, was used for protein intensity comparisons. False discovery rate correction and log-ratio threshold were set to $\leq 0.09$ (Schwämmle et al., 2020). All detected proteins were searched against the UniProtKB database using DIAMOND (Buchfink et al., 2014), with a E-value $\leq 1 \times 10^{-5}$. The top manually reviewed SwissProt matches were used to assign protein sequence IDs and annotations, unless no SwissProt matches were detected, where TrEBML matches were assigned instead. Any unmatched sequences were designated as hypothetical proteins.

\subsection{Results and Discussion}

\subsubsection{Colonisation Success}

Genotyping confirmed the genetic identity of the symbionts, both at the beginning and end of the experiment. However, symbiont cell densities were different between species by Week 14 (one-way ANOVA, $F_{1,14}=11.816, P<0.05$ ), with $B$. minutum having a 1.5 -fold higher population density $\left(5.47 \times 10^{6} \pm 2.53 \times 10^{5} \mathrm{SE}\right.$ cells $\mathrm{mg}^{-1}$ host protein) than $D$. trenchii $(3.55$ $\times 10^{6} \pm 4.97 \times 10^{5} \mathrm{SE}$ cells $\mathrm{mg}^{-1}$ host protein) (Fig. 3.1). Both B. minutum and D. trenchii reach a steady state eight weeks after colonization, with $B$. minutum having higher population densities than D. trenchii (Gabay et al., 2019, 2018; Sproles et al., 2020, 2019). This is consistent with the findings from the current study. 


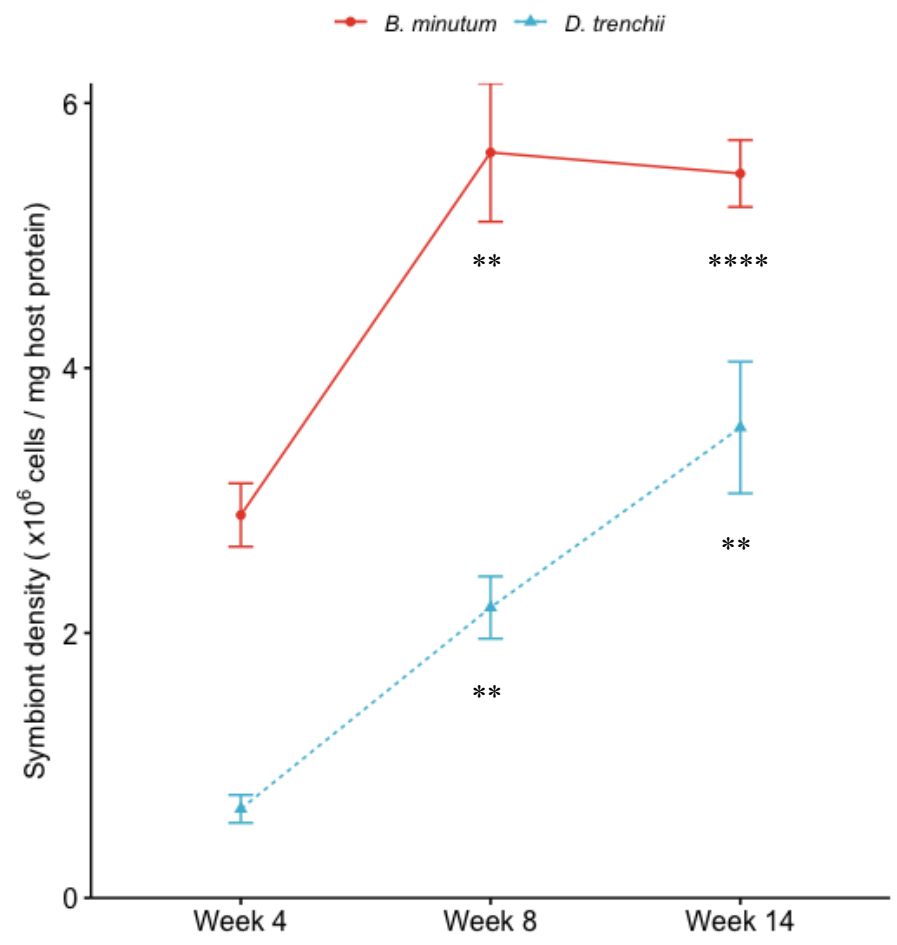

Figure 3.1. Population density of different symbiont species (Breviolum minutum and Durusdinium trenchii) in Aiptasia, at different time-points during 14 weeks of colonisation. Asterisks indicate significant differences between each time-point $v s$. Week 4: $* * \mathrm{p}<0.01, * * * \mathrm{p}<0.001, * * * * \mathrm{p}<0.0001$. Values are mean $\pm \mathrm{SE} ; \mathrm{n}$ $=8$ per symbiont species per time-point.

\subsubsection{Differentially expressed proteins in B. minutum vs. D. trenchii}

A total number of 1720 and 1536 proteins were detected across all colonization time-points for B. minutum and D. trenchii, of which 27 and 21 false matches were discarded, respectively. In total, 336 and 684 proteins (Tables S3.1-S3.5) were differentially expressed across all timepoints in B. minutum and D. trenchii, respectively. Of these, 331 and 668 were annotated based on homology with sequences in the UniProtKB database $\left(\right.$ E-value $\left.\leq 1 \times 10^{-5}\right)$ and the remaining 21 sequences were designated as hypothetical proteins.

Generally, the observed proteomes were characteristic of symbiotic state (i.e., ex or in hospite), colonization stage, and symbiont identity, with symbiotic state in particular having a major influence, irrespective of symbiont species. During colonization by B. minutum, 179 symbiont proteins were significantly different between Weeks 0 vs. 4; there were fewer changes afterwards, with 54 differentially expressed proteins between Weeks 4 vs. 8, and only four differentially expressed proteins between Weeks 8 vs. 14. Ultimately, there were 100 
differentially expressed proteins between the start and end of colonisation (i.e., Weeks 0 and 14). Similarly, PCA plots showed that the B. minutum proteome changed markedly during colonization, with the most distinct profile being observed at Week 4 (Fig. 3.2A). Likewise, the $D$. trenchii proteomes at the start and end of the experiment were markedly different from each other (Fig. 3.2B).
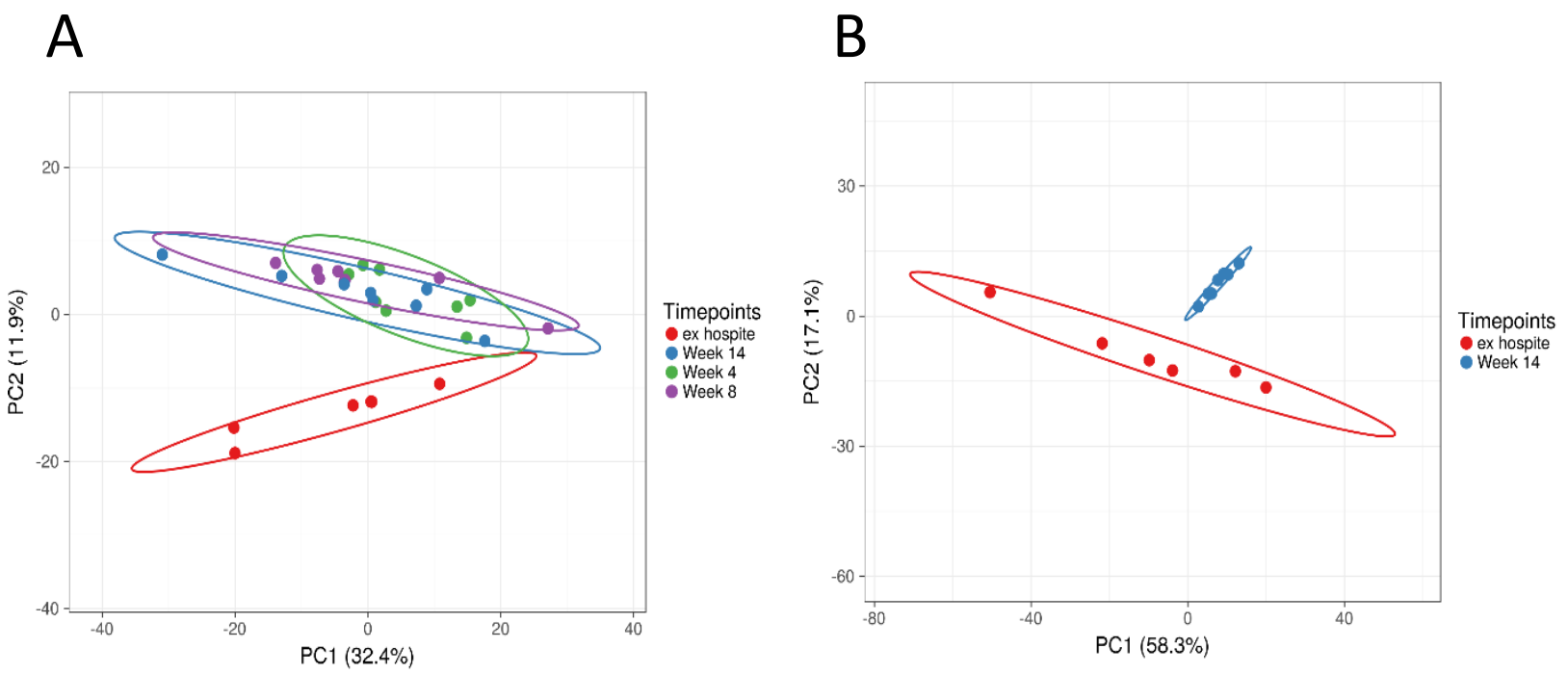

Figure 3.2 Principal component analysis plots of all detected symbiont proteins at different times (Week 0, i.e. ex hospite, and Weeks 4, 8, and 14 post-inoculation) during colonization of Aiptasia by (A) Breviolum minutum and (B) Durusdinium trenchii. Grouped by ellipses with $99 \%$ confidence intervals.

Changes in the B. minutum proteome during colonization were consistent with the increasing symbiont population density and the modifications required to ensure persistence of the symbionts inside the host's cells. In particular, differentially expressed proteins included those with predicted roles in: acquisition of inorganic carbon (DIC); nitrogen metabolism; microand macronutrient starvation (e.g., phosphorus); photosynthesis; suppression of host immune responses; tolerance of low $\mathrm{pH}$; and management of oxidative stress. All these proteins are discussed below, in terms of the changes seen during colonization by the native symbiont and the differences with the non-native D. trenchii (Tables 3.1 and 3.2).

\subsubsection{B. minutum proteome changes over the course of host colonization}

Most of the symbiont proteins that were differentially expressed during colonization by $B$. minutum were linked to photosynthesis. Homologues of light-harvesting proteins of photosystems I and II, such as fucoxanthin-chlorophyll $a-c$ binding protein (FCB) and 
peridinin-chlorophyll $a$-binding protein (PCP) were identified in B. minutum across all timepoints and were upregulated during colonization. PCPs and FCBs are among the major lightharvesting proteins of Symbiodiniaceae, with high performance capability under fluctuating light conditions (Boldt et al., 2012; Nagao et al., 2013; Ueno et al., 2019). The amount of FCBs has been shown to change in response to different light intensity in marine algae. For instance, in a study of different FCBs, the amount of FCB2 increased under low-light while FCB4 quantity did not show any change; in contrast, FCB6 was more abundant under high light (Becker and Rhiel, 2006). PCPs also increase in Symbiodiniaceae when in hospite vs ex hospite (Stochaj and Grossman, 1997). Consistent with this, both FCBs and PCPs in B. minutum were upregulated at Week $8 v$ s. Week 4, peaking at the same time as the symbiont population density. An increase in pigmentation as a result of symbiont self-shading is a light-adaptive strategy, so the observed patterns were unsurprising (Kahng et al., 2010; Kaiser et al., 1993). Also unsurprising was that this increase in light-harvesting proteins was coupled with an upregulation of other proteins involved in photosynthesis. These included chloroplastic electron transport proteins, such as cytochromes, flavodoxin, PSI reaction centre proteins and ferredoxin-NADP reductase, as well as proteins associated with the reductive pentosephosphate cycle (e.g., phosphoribulokinase), the first step of carbon fixation (Nickelsen, 2015) (Table 3.1). Furthermore, photosynthesis increases protein synthesis by phosphorylating ribosomal proteins (Reinbothe et al., 2010; Tcherkez et al., 2020). Similarly, proteins involved in translation, protein folding, chloroplast maintenance and photosystem repair were upregulated at Week 8. Increased translation of photosynthesis repair machinery in symbiotic Symbiodiniaceae during thermal stress and increased photosynthesis have been reported before (Bhagooli, 2013; Takahashi et al., 2004), so upregulation of these proteins might simply be due to higher rates of photosynthesis.

Two homologues of general transmembrane transporter, ABC ATP-binding protein were upregulated at Week 8. ABC transporters are members of the most abundant superfamily of transporters, with a broad range of transported compounds (Dassa and Bouige, 2001). These transporters have been reported in different Symbiodiniaceae, with ABC genes being abundant in the B. minutum genome (Aranda et al., 2016). Moreover, host ABC transporters have been identified in symbiosome extracts of Aiptasia (Peng et al., 2010). However, whether algal ABC transporters are involved in extracellular or intracellular transport remains to be determined. Finally, a homologue of 14-3-3- protein (GF14), which is involved in the response to cellular 
nitrogen starvation by controlling GS and nitrate reductase via phosphorylation, was also upregulated at Week 8 (Comparot et al., 2003). As previously noted, nitrogen limitation is a primary mechanism for regulating symbiont density by the host. Thus, upregulation of GF14 at Week 8, when B. minutum reached its maximum population density, might be linked to the host's need to regulate symbiont proliferation.

As mentioned before, only four proteins were differentially expressed between Weeks 8 and 14 (Table S3.3). One of these was a homologue of oryzain alpha chain (OAC), which was upregulated 1.46-fold $\left(q \leq 4.9 \times 10^{-2}\right)$. OAC is expressed in response to a wide range of stressors, and also induces host cell necrosis in a plant-parasitic fungus association (Fu et al., 2007). The upregulation of OAC at Week 14 might therefore contribute to the symbiont population density at this point, though this requires further research.

\subsubsection{Comparative responses of $B$. minutum vs. D. trenchii}

\subsubsection{Oxidative stress}

Homologues of algal antioxidant network proteins were found in both B. minutum and $D$. trenchii. Reactive oxygen species (ROS) are oxidants that can damage DNA, proteins and cellular membranes (Downs et al., 2002; Venn et al., 2008). The dinoflagellate symbiont's antioxidant network, in response to increased ROS under high temperature and irradiance, has been well studied in the past (Krueger et al., 2014; McGinty et al., 2012; Roberty et al., 2016; Wietheger et al., 2018), though ROS are also regular by-products of photosynthesis and photorespiration; antioxidant defences are therefore needed at all times. Here, homologues of symbiont superoxide dismutase (SOD) and ascorbate peroxidase (APX), which work in concert to detoxify superoxide and hydrogen peroxide, were detected in B. minutum at all time-points, with both being upregulated progressively during colonization of the host (Table 3.1). This is unsurprising, as photosynthetic activity by the symbionts can rapidly induce a state of hyperoxia in the host's tissues (Richier et al., 2003). Homologues of APX and SOD, as well as other antioxidant network proteins such as glutathione reductase and cytochrome $c$ peroxidase, were also detected in $D$. trenchii, however contrary to B. minutum, these proteins were downregulated when in hospite (Table S3.5). The reasons for this are unclear, but this was coupled with downregulation of proteins involved in photosynthesis. D. trenchii has been 
previously reported to have reduced photosynthetic incorporation of ${ }^{13} \mathrm{C}$ per cell in comparsion to B. minutum when in symbiosis with Aiptasia (Sproles et al., 2020). Therefore, downregulation of the antioxidant network in this species might be associated with reduced levels of photosynthesis.

\subsubsection{Dissolved inorganic carbon transport}

Three and 16 homologues of symbiont carbonic anhydrases (CAs) were upregulated in freeliving B. minutum and D. trenchii, respectively. All three homologues from B. minutum were exclusive to the free-living state, while $12 \mathrm{CA}$ homologues from $D$. trenchii were uniquely expressed ex hospite (Table 3.2). Dissolved inorganic carbon (DIC) is crucial for both photosynthesis and calcification, however DIC in ambient seawater is mostly present as bicarbonate $\left[\mathrm{HCO}_{3}^{-}\right]$, which needs to be converted to carbon dioxide $\left[\mathrm{CO}_{2}\right]$ to freely pass through cell membranes. Therefore, the cnidarian host actively facilitates DIC uptake via carbon concentrating mechanisms (CCMs), a process during which bicarbonate is converted to carbon dioxide by host carbonic anhydrase (CA) (Allemand et al., 1998; Furla et al., 2000b). Proton pumps are also part of CCMs, acidifying the ambient seawater to form more carbonic acid for CA (Furla et al., 2000a). Cnidarian CAs and proton pumps are significantly upregulated upon symbiosis establishment to supply the intracellular symbionts with DIC (Oakley et al., 2016; Sproles et al., 2019). Dinoflagellate genes encoding proton pumps in dinoflagellate-cnidarian and -bivalve symbioses also form part of CCMs (Bertucci et al., 2010; Mies et al., 2017), however homologues of proton pumps, like V-type proton ATPase, were only found in free-living D. trenchii $\left(q \leq 2.3 \times 10^{-2}\right)$. Upregulated algal CAs and proton pumps in the free-living state might be a response to a lack of readily available $\mathrm{CO}_{2}$ provided by the host CAs and proton pumps (Barott et al., 2015b; Furla et al., 2000a; Oakley et al., 2016; Sproles et al., 2019). 


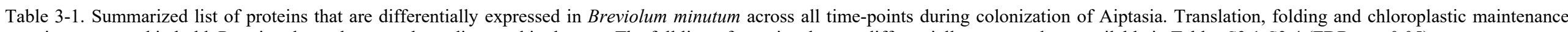
proteins are named in bold. Proteins shown here are those discussed in the text. The full lists of proteins that are differentially expressed are available in Tables S3.1-S3.4 (FDR, q $<0.05$ ).

\begin{tabular}{|c|c|c|c|c|c|}
\hline UniProtKB ID & UniProtKB Protein Names & $\begin{array}{l}\text { log-ratios Zero vs Week4 } \\
(q \text {-value })\end{array}$ & $\begin{array}{l}\text { log-ratios Week } 4 \text { vs Week } 8 \\
(q \text {-value })\end{array}$ & $\begin{array}{l}\text { log-ratios Week } 8 \text { vs Week } 14 \\
(q \text {-value })\end{array}$ & $\begin{array}{l}\text { log-ratios Zero vs Week } 14 \\
(q \text {-value })\end{array}$ \\
\hline $\mathrm{P} 42644$ & 14-3-3-like protein GF14 psi & - & $-0.43(2.5 \mathrm{E}-06)$ & - & \\
\hline A0T0C6 & Cytochrome $c-550$ & $1.05(4.2 \mathrm{E}-03)$ & $-0.75(4.2 \mathrm{E}-04)$ & - & \\
\hline P00110 & Cytochrome $c 6$ & $1.03(4.2 \mathrm{E}-02)$ & $-0.92(9.7 \mathrm{E}-04)$ & - & \\
\hline A2Y8E0 & Ferredoxin--NADP reductase (FNR) & $-1.96(2.1 \mathrm{E}-04)$ & $-0.50(2.5 \mathrm{E}-03)$ & -2 . & $(2.2 \mathrm{E}-05)$ \\
\hline P14070 & Flavodoxin & $2.45(8.8 \mathrm{E}-08)$ & $-1.22(1.8 \mathrm{E}-03)$ & 1.2 & 2.7E-05) \\
\hline P09195 & Fructose-1,6-bisphosphatase (FBPase) & - & - & $1.23(4.5 \mathrm{E}-03)$ & \\
\hline Q40297 & $\begin{array}{l}\text { Fucoxanthin-chlorophyll } a-c \text { binding protein A } \\
\text { (FCB) }\end{array}$ & $0.85(2.2 \mathrm{E}-03)$ & $-0.32(5.0 \mathrm{E}-03)$ & -0 . & $(2.7 \mathrm{E}-05)$ \\
\hline O09452 & Glyceraldehyde-3-phosphate dehydrogenase & - & - & $0.66(3.4 \mathrm{E}-02)$ & \\
\hline Q9STW6 & Heat shock 70 kDa protein 6 & $1.15(6.2 \mathrm{E}-04)$ & $-0.79(3.6 \mathrm{E}-03)$ & 0.5 & (4.6E-02) \\
\hline O80796 & Membrane-associated protein VIPP1 & - & $-0.51(4.1 \mathrm{E}-02)$ & - & \\
\hline P25776 & Oryzain alpha chain & - & - & $-1.46(4.9 \mathrm{E}-02)$ & \\
\hline A0A1Q9EDY9 & Pentatricopeptide repeat-containing protein & - & $-0.8(3.1 \mathrm{E}-06)$ & - & \\
\hline P51874 & Peridinin-chlorophyll $a$-binding protein (PCP) & - & - & $-0.35(4.6 \mathrm{E}-04)$ & \\
\hline P80483 & Peridinin-chlorophyll $a$-binding protein 3 (PCP) & $1.83(9.9 \mathrm{E}-09)$ & $-0.80(1.2 \mathrm{E}-07)$ & - & \\
\hline P26302 & Phosphoribulokinase, (PRK) & - & $-0.87(8.8 \mathrm{E}-03)$ & -0 . & $(1.8 \mathrm{E}-02)$ \\
\hline P49481 & Photosystem I reaction centre subunit II (PSI-D) & $-0.50(9.9 \mathrm{E}-04)$ & $-0.63(4.2 \mathrm{E}-04)$ & -1 . & $(1.2 \mathrm{E}-06)$ \\
\hline Q7XJ02 & Probable L-ascorbate peroxidase 7 & - & - & -1 . & $(2.6 \mathrm{E}-03)$ \\
\hline A0A1Q9EFF8 & Protein GrpE & - & $-0.33(3.4 \mathrm{E}-02)$ & - & \\
\hline A0A1Q9D599 & Putative $\mathrm{ABC}$ transporter ATP-binding protein & $-0.81(3.0 \mathrm{E}-03)$ & $-1.08(1.6 \mathrm{E}-06)$ & -1 . & $(1.2 \mathrm{E}-06)$ \\
\hline A0A1Q9D599 & Putative $\mathrm{ABC}$ transporter ATP-binding protein & $-0.81(3.0 \mathrm{E}-03)$ & $-0.76(1.9 \mathrm{E}-04)$ & -1 . & $(1.2 \mathrm{E}-06)$ \\
\hline Q8H0M1 & Quinone-oxidoreductase homolog, (ceQORH) & - & $-0.44(3.7 \mathrm{E}-02)$ & - & \\
\hline P54375 & Superoxide dismutase & $2.12(1.0 \mathrm{E}-04)$ & $-0.91(1.8 \mathrm{E}-03)$ & - & \\
\hline A0A1Q9DKM4 & Tyrosyl-DNA phosphodiesterase 2 & - & $-1.03(1.2 \mathrm{E}-07)$ & - & \\
\hline P68202 & Ubiquitin-40S ribosomal protein $\mathrm{S27a}$ & - & $-0.67(7.6 \mathrm{E}-04)$ & - & \\
\hline
\end{tabular}




\subsubsection{Nitrogen metabolism}

A group of proteins with predicted function in dissolved inorganic nitrogen (DIN) uptake and metabolism were identified in both species both at the start and end of the experiment. Nitrogen is an essential macronutrient, required by both dinoflagellate symbionts and their cnidarian hosts (Cook et al. 1988; Miller and Yellowlees, 1989; Rädecker et al., 2017). Ammonium $\left(\mathrm{NH}_{4}{ }^{+}\right)$and nitrate $\left(\mathrm{NO}_{3}{ }^{-}\right)$are the most available forms of DIN in seawater, with ammonium being more preferred over nitrate by symbiotic cnidarians (Grover et al., 2008). Both partners can assimilate DIN, however the symbionts are the primary site of DIN uptake, with assimilation occurring via the glutamine synthetase/glutamine 2-oxoglutarate amido transferase (GS/GOGAT) pathway. This pathway generates glutamate as the final product, which is a precursor for other amino acids or purines/ureides. In addition, the algal symbionts can assimilate ammonium via glutamate dehydrogenase (GDH), though this has a lower affinity for ammonium than does GS/GOGAT (Grover et al., 2002; Miller and Yellowlees, 1989; Pernice et al., 2012; Roberts et al., 2001; Summons and Boag, 1986; Wilkerson and Muscatine, 1984; Yellowlees et al., 1994). In the present study, different homologues of GS/GOGAT were isolated from both symbiont species, while a homologue of GDH was only present in symbiotic B. minutum. However, in B. minutum, these enzymes were not differentially expressed either ex hospite or in hospite, or between the different time-points during colonization. In contrast, in D. trenchii, four and two homologues of GS and GOGAT, respectively, were upregulated ex hospite relative to the in hospite state. Nitrogen limitation has been suggested as the primary mechanism by which the host controls the proliferation of its symbionts (Cook et al., 1988; McAuley and Cook, 1994; Rahav et al., 1989; Wang and Douglas, 1999; Yellowlees et al., 1994), so this might explain the lower expression of GS/GOGAT in symbiotic $D$. trenchii, as well as the more consistent expression of these enzymes in B. minutum.

Regardless of the assimilatory mechanism, assimilated nitrogen is used as a precursor for de novo synthesis of amino acids and nucleotides (Newsholme et al., 2003). Consistent with this, a group of proteins involved in methionine synthesis and metabolism were found in both algal species. Methionine is important in the cnidarian-dinoflagellate symbiosis, with both partners having specific metabolic pathways (Shinzato et al., 2014). Here, D. trenchii showed upregulation of methionine and S-adenosylmethionine synthase $\left(q \leq 2.7 \times 10^{-2}\right.$ and $q \leq 2.5 \times$ 67 
$10^{-2}$, respectively) only in the free-living state, while $B$. minutum showed upregulation of Sadenosylmethionine synthase in symbiosis (1.31-fold, $\left.q \leq 1.4 \times 10^{-6}\right)$. S-adenosylmethionine synthase is a very efficient enzyme that contributes to methionine recycling (Fontecave et al., 2004). This pattern could help to explain the observation of Sproles et al. (2019) and Matthews et al., 2017, who noted that Aiptasia likely receives more methionine from $B$. minutum than $D$. trenchii, based on proteomic and metabolomic evidence. Additionally, a homologue of guanosine monophosphate synthetase (GMP), with a predicted function of de novo synthesis of guanine nucleotides by hydrolysing glutamine (Nakamura et al., 1995), was found to be upregulated at Week 14 in B. minutum $\left(q \leq 7.6 \times 10^{-3}\right)$. The alternative fate of glutamine in the symbionts is its storage in high-nitrogen compounds via the purine pathway (Pernice et al., 2012). Therefore, upregulation of GMP in symbiotic B. minutum, while being absent from in and ex hospite D. trenchii, might imply better cellular integration of the native symbionts with their host, as opposed to the less well integrated, more opportunistic characteristics of the nonnative $D$. trenchii.

\subsubsection{Requirements for living inside the symbiosome}

In cnidarian-dinoflagellate associations, the endosymbionts are located in a host-derived late arrested phagosome, the 'symbiosome', which acts as the interface between the partner organisms (Fitt and Trench, 1983; Hohman et al., 1982; Neckelmann and Muscatine, 1983; Wakefield et al., 2000; Wakefield and Kempf, 2001). Inter-partner communication occurs across the symbiosome membrane complex, as described in detail by Rosset et al. (2021), while the symbionts must cope with the environmental conditions imposed by living inside the symbiosome. Here, extracellular and cell surface proteins were abundant in the symbiotic state. For instance, a homologue of acyl coenzyme A binding protein (ACBP) was found to be consistently expressed from Week 4 onwards only by B. minutum $\left(q \leq 4.6 \times 10^{-4}\right)$. ACBP's role in intracellular lipid trafficking has been well described in the past (Cabral et al., 2010). However, the ACBP homologue detected here is putatively located at the extracellular matrix (ECM) (e.g., cell wall), with predicted functions in cell morphogenesis and differentiation and signalling, and the provision of structural support and protection (Cabral et al., 2010). Of note, extracellular ACBP is increasingly expressed under abiotic stress, such as nitrogen limitation and low pH (Duran et al., 2010; Manjithaya et al., 2010; Qiao et al., 2018), both of which are characteristic of the conditions experienced by symbionts inside the symbiosome (Barott et al., 68 
2015b; Wang and Douglas, 1999, 1998) and hence may contribute to the increased in hospite expression of ACBP seen in the current study.

A homologue of extracellular purple acid phosphatase (PAP) was found to be exclusively expressed by $B$. minutum when in hospite $\left(q \leq 1.7 \times 10^{-2}\right)$, though it had similar expression levels across all colonization time-points. Extracellular PAPs play a role in inorganic phosphate (Pi) acquisition and scavenging during phosphate starvation by hydrolysing organophosphate compounds, as has been previously reported for both plant-microbe symbioses and free-living marine diatoms (Li et al., 2012; Robinson et al., 2012; Wang et al., 2021). Interestingly, PAPs are localized to the symbiosome surface in plant-microbe symbioses, as well as the cnidariandinoflagellate symbiosis (Ezawa et al., 2005; Penheiter et al., 1997; Rands et al., 1993; Robinson et al., 2012). Additionally, two acid phosphatases involved in carrier-mediated phosphate transportation, have previously been reported in the coral Acropora formosa, one of which is external to the algal plasmalemma and is involved in phosphate ester translocation from the cnidarian cell cytoplasm into the symbiosome (Jackson et al., 1989). However, the phosphate uptake mechanism of the PAP homologue identified in the current study remains to be investigated.

Furthermore, two homologues of glycerophosphoinositol permease 1 (GIT1) were expressed progressively more during colonization in B. minutum, being most abundant at Week 14 ( $q \leq$ $1.4 \times 10^{-3}$ ). GIT1 facilitates glycerophosphoinositol (GPI), a product of phosphatidylinositol deacylation, transportation across the cell membrane. GPI can be used as a source of phosphate, especially during phosphate starvation or in acidic environments (Almaguer et al., 2004; Bishop et al., 2011; P. Ziegler et al., 2017), both of which are once again likely features of the intra-symbiosome environment (Barott et al., 2015b; Jackson et al., 1989). These proteins (i.e., PAP and GIT1) were not detected at all in D. trenchii, suggesting either that this symbiont species uses a different mechanism for sourcing phosphate or that host-symbiont cellular integration is sub-optimal in this association, as occurs in other physiological respects when $D$. trenchii colonizes Aiptasia (Matthews et al., 2017; Gabay et al., 2018). 
Another cell surface protein detected was a homologue of arylsulfatase B (ASB), which has a predicted function in autophagy and metabolization of sulphated polysaccharides (Kamke et al., 2013; Slaby et al., 2017). ASB was not observed ex hospite and but was upregulated by $B$. minutum during colonization, with its maximum expression at Week $14\left(q \leq 9.2 \times 10^{-4}\right)$. In marked contrast, in D. trenchii, ASB was detected both ex hospite and after 14 weeks in symbiosis, though was in fact more expressed when in the non-symbiotic state $(2.88$-fold, $q \leq$ $\left.1.3 \times 10^{-3}\right)$. In adults of the coral Montastraea faveolata, increased ASB levels in host tissues are thought to help regulate the symbiont population via autophagy, while conversely, levels of this protein are reduced in larvae of the coral Porites astreoides, potentially in response to low symbiont numbers and the need for the symbionts to proliferate (O'Rourke and Rourke, 2011; Walker et al., 2019). Whether the ASB detected in the two algal species here is similarly involved with autophagy and hence self-control of the symbiont population is unknown, though the coordination of host-symbiont biomass is important from the perspective of both partners (Davy et al., 2012). Alternatively, given the high expression of ASB in the non-symbiotic state of $D$. trenchii, it is plausible that the symbionts employ ASB for heterotrophy on sulphated polysaccharides, given the heterotrophic potential of these dinoflagellates (Jeong et al., 2012; Sproles et al., 2020).

A group of homologues of Ras-related proteins (e.g. RAB1 and RAB34) were found to be differentially expressed between symbiotic states (i.e., ex and in hospite) of both B. minutum and D. trenchii. Cnidarian Rab family proteins are known to be upregulated after symbiont inoculation; and are responsible for symbiosome formation and symbiont density regulation via endocytosis, and exocytosis or phagocytosis of dysfunctional endosymbionts (Chen et al., 2005, 2004, 2003; Daniels et al., 2015; Fransolet et al., 2012; Mohamed et al., 2016; Yoshioka et al., 2021). Rab proteins have also been reported from the symbionts of the coral Stylophora pistillata, once again functioning in exocytosis (Weston et al., 2012). In addition, manipulation of the symbiosome membrane by algal Rab proteins has been suggested, where some of algal Rab1-7 transcripts from two different species of Symbiodiniaceae - D. trenchii and Cladocopium goreaui - were upregulated during colonization of larvae of the coral Acropora tenuis (Yuyama et al., 2018). In the current research, homologues of Rab34 from B. minutum and D. trenchii were higher in the ex hospite vs. in hospite state (2.99-fold, $q \leq 1.9 \times 10^{-5}$ and 4.6-fold, $q \leq 4.1 \times 10^{-16}$, respectively), whereas Rab1 homologues were upregulated at Week 
14 of colonization in both species (Table 3.2). However, Rab1 was highly abundant in $B$. minutum at Week 14 while D. trenchii exhibited a lesser degree of Rab1 upregulation. Notably, this increase in Rab 1 expression coincided with a decline in the B. minutum population, unlike the D. trenchii population which was still growing at this stage (Fig. 3.1). However, the exact role of RAB1 in the regulation of symbiont biomass remains unclear.

Table 3-2. Summarized list of proteins that are differentially expressed in Breviolum minutum and Durusdinium trenchii when comparing ex hospite cells vs in hospite cells after 14 weeks of colonization of Aiptasia. Proteins shown here are those discussed in the text. The full lists of proteins that are differentially expressed between the ex hospite state (Week 0) and Week 14 of colonization are available in Tables S3.4 and S3.5 (FDR, q $<0.05$ ).

\begin{tabular}{|c|c|c|c|}
\hline UniProtKB ID & UniProtKB Protein Names & $\begin{array}{l}\text { D. trenchii Weeks } 0 \text { vs } 14 \\
\text { fold change } \\
(q \text {-value })\end{array}$ & $\begin{array}{l}\text { B. minutum Weeks } 0 \text { vs } 14 \text { fold } \\
\text { change } \\
\text { (q-value })\end{array}$ \\
\hline Q5FXM5 & Acyl-CoA-binding protein (ACBP) & - & Unique, in hospite (4.6E-04) \\
\hline P50430 & Arylsulfatase B (ASB) & $2.88(1.3 \mathrm{E}-03)$ & Unique, in hospite (9.2E-04) \\
\hline A4UHC0 & Calmodulin $(\mathrm{CaM})$ & $-0.36(1.3 \mathrm{E}-02)$ & - \\
\hline Q9I262 & Carbonic anhydrase & Unique, ex hospite (4.8E-03) & Unique, ex hospite (3.4E-04) \\
\hline Q5BCC5 & Carbonic anhydrase & Unique, ex hospite (4.0E-04) & - \\
\hline A0A1Q9D9X1 & Carbonic anhydrase 2 & - & Unique, ex hospite (1.9E-04) \\
\hline A0A1Q9CS65 & Carbonic anhydrase 2 & Unique, ex hospite (1.9E-02) & Unique, ex hospite (4.8E-02) \\
\hline Q59Q30 & $\begin{array}{l}\text { Glycerophosphoinositol permease } \\
1 \text { (GIT1) }\end{array}$ & - & Unique, in hospite (1.4E-03) \\
\hline B4R9R7 & $\begin{array}{l}\text { GMP synthase (Glutamine } \\
\text { amidotransferase) }\end{array}$ & - & Unique, in hospite (7.6E-03) \\
\hline P62785 & Histone $\mathrm{H} 4$ variant (TH011) & $-11.26(2.2 \mathrm{E}-124)$ & - \\
\hline Q7MHB1 & Methionine synthase (MS) & Unique, ex hospite (2.7E-02) & - \\
\hline P34791 & $\begin{array}{l}\text { Peptidyl-prolyl cis-trans isomerase } \\
\text { (PPIase) }\end{array}$ & $-4.30(2.7 \mathrm{E}-06)$ & 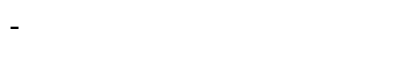 \\
\hline Q9LXI4 & Purple acid phosphatase 21 (PAP) & - & Unique, in hospite (1.7E-02) \\
\hline A0A1Q9F5Z1 & Ras-related protein (ORAB-1) & - & Unique, in hospite (1.9E-03) \\
\hline P62822 & Ras-related protein (Rab-1A) & $-0.98(4.6 \mathrm{E}-05)$ & - \\
\hline A0A1Q9EXN6 & Ras-related protein (Rab-34) & $4.60(4.1 \mathrm{E}-16)$ & $2.99(1.9 \mathrm{E}-05)$ \\
\hline A6XMY9 & $\begin{array}{l}\text { S-adenosylmethionine synthase } 1 \\
\text { (MAT 1) }\end{array}$ & - & $-1.31(1.4 \mathrm{E}-06)$ \\
\hline A9NUH8 & $\begin{array}{l}\text { S-adenosylmethionine synthase } 1 \\
\text { (MAT 1) }\end{array}$ & $1.01(2.5 \mathrm{E}-02)$ & - \\
\hline P20654 & $\begin{array}{l}\text { Serine/threonine-protein } \\
\text { phosphatase (PP1) }\end{array}$ & Unique, in hospite (1.3E-02) & - \\
\hline O00780 & V-type proton ATPase subunit E & Unique, ex hospite (2.3E-02) & - \\
\hline
\end{tabular}

\subsubsection{Immunosuppression}

Most of the differentially expressed proteins isolated from $D$. trenchii when in hospite had predicted functions associated with host cell immunosuppression while none of these proteins had differential expression in B. minutum. For example, a homologue of peptidyl-prolyl cistrans isomerase (PPIase) was upregulated at Week 14 in D. trenchii $\left(4.3\right.$-fold, $\left.q \leq 2.7 \times 10^{-6}\right)$. The isomerase activity of PPIase is thought to facilitate the proper folding of specific proteins (Galat, 1993; Perez and Weis, 2008), however PPIase can also play an important role in host- 
microbe associations, such as host entry/infection, as described in detail by Ünal and Steinert (2014). In the cnidarian-dinoflagellate symbiosis, dose-dependent inhibition of host PPIase has been shown to result in symbiont expulsion from Aiptasia (Perez and Weis, 2008), so it is conceivable that upregulation of symbiont PPIase activity by $D$. trenchii when in symbiosis might reflect efforts by the symbiont to avoid expulsion by the host. In addition, a homologue of serine/threonine-protein phosphatase (PP1) was exclusively expressed by $D$. trenchii when in hospite $\left(q \leq 1.3 \times 10^{-2}\right)$. Serine/threonine-protein phosphatases/kinases, which are dependent on calcium/calmodulin (CaM) mediated signalling, are important in cell signalling and proliferation. Moreover, CaM-dependent PP1 activity is involved in immune pathways, and PP1 is upregulated during the establishment of other symbioses (Klee et al., 1998). For example, the inhibition of PP1 in Aiptasia has been shown to be coupled with oxidative stress and symbiont expulsion (Perez and Weis, 2008), while the transcriptional regulation of PP1 is known to increase specifically in apicomplexan parasites (Plasmodium) upon host infection (Dobson et al., 2001). Interestingly, a homologue of CaM was upregulated in the current study alongside PP1 (0.36-fold, $\left.q \leq 1.3 \times 10^{-2}\right)$, consistent with a degree of functional co-dependency. PP1 from Symbiodiniaceae has been observed to be upregulated under different environmental stresses, such as low phosphorus availability and $\mathrm{pH}$, and increased temperature (Gierz et al., 2017; Lin et al., 2018; Shi et al., 2017), however in the current study it seems more likely that increased PP1 expression might form part of a strategy for suppressing the host's immune responses.

Finally, a homologue of histone $\mathrm{H} 4$ was identified in both ex hospite and in hospite D. trenchii, though it was most highly upregulated at Week 14 post-inoculation (11.26-fold, $q \leq 2.2 \times 10^{-}$ $\left.{ }^{124}\right)$. Histones pack DNA in all eukaryotic organisms, however histones can undergo a large number of post-translational modifications, some of which are thought to be important in parasites and virulence (Saraf et al., 2016). In particular, histone H4, from a range of pathogenic and virulent associations, is responsible for suppressing host cell development and immunity in an epigenetic manner (Chen et al., 2016; Gad and Kim, 2009, 2008; Kim and Kim, 2010; Kumar et al., 2017; Saraf et al., 2016). Detailed examination of these proteins is required, however, to decipher their role in the suppression of host immunity by $D$. trenchii. 


\subsection{Conclusion}

This study identified differentially expressed proteins in B. minutum during colonization of its native host, the model cnidarian Aiptasia. In particular, proteins involved in photosynthesis/respiration, nutrient exchange, oxidative stress and cell apoptosis increased as colonization progressed, with most of these changes likely linked to the increasing symbiont cell density. This study also compared the proteomes of B. minutum and the non-native $D$. trenchii after full colonization of Aiptasia. The most distinct difference between these symbiont species related to their cell surface proteins, some of which were not detectable in D. trenchii. Moreover, B. minutum exhibited an upregulation of proteins associated with nutrient limitation (consistent with host-regulated nutrient supply), while the majority of proteins upregulated in D. trenchii have potential roles in host immunosuppression. Taken together, these observations are consistent with the view that B. minutum forms a well-integrated, functional symbiosis with Aiptasia, while $D$. trenchii does not, reflecting its reputation as a more metabolically-costly opportunist (Gabay et al., 2018; Matthews et al., 2018, 2017; Pettay et al., 2015; Sproles et al., 2019; Stat and Gates, 2010). The acquisition of more thermally tolerant symbionts such as $D$. trenchii has been suggested as a coping strategy during environmental stress (Cunning et al., 2015b; Reich et al., 2017). However, this current study adds to the ever increasing body of evidence that host-symbiont specificity, and its consequences for symbiotic integration and holobiont function, will limit such opportunities (Gabay et al., 2019). Further research is now needed on the effects of time and temperature on the various host-symbiont interactions, to determine whether inter-partner integration and function in novel symbioses might improve over time, so enabling us to better understand the response of coral reefs to climate change. 


\section{Chapter 4 \\ Immunolocalization of metabolite transporter proteins in a model cnidarian-dinoflagellate symbiosis}

\subsection{Introduction}

Corals reefs are one of the most prominent, productive, and diverse ecosystems in the world's oceans. They provide different ecological services, such as a three-dimensional sheltered habitat for other marine life-forms. Corals' success is based upon on obligatory endosymbiotic relationship between the cnidarian host and its dinoflagellate algal partners (family: Symbiodiniaceae) (Muscatine and Porter, 1977; Polovina, 1984; Sheppard et al., 2018). Bidirectional nutrient flow has a crucial role in this endosymbiosis, with the cnidarian host being responsible for supplying inorganic carbon, nitrogen, phosphorus and other nutrients to the algae, while the endosymbionts provide the host with photosynthetic products (Davy et al., 2012). This exchange facilitates the survival and proliferation of coral reefs in nutrient-poor tropical seas.

Glycerol was originally considered to be the main form in which photosynthetically-fixed carbon is translocated from symbiont to host (Muscatine, 1967, 1965), though more recent studies have shown the prominent trafficking of glucose from the endosymbiont to the host (Burriesci et al., 2012; Hillyer et al., 2017b; Suescún-Bolívar et al., 2016). Other constituents of the translocated photosynthate include fatty acids such as palmitic, stearic and oleic acids, lipids like wax esters, triacylglycerol and sterols (Dunn et al., 2012; Hillyer et al., 2016; Yamashiro et al., 1999). Nitrogen exchange is also crucial to the symbiosis, with ammonium being especially significant, as this is the primary form in which cnidarians excrete nitrogenous waste and is easily assimilated by the algal symbionts (Ezzat et al., 2017; Muller-Parker and Hoegh-Guldberg, 1994; Pernice et al., 2012). Symbionts may then metabolize or store assimilated nitrogenous compounds, and return a portion to the host as amino acids, creating an efficient recycling pathway that benefits the symbiosis (Kopp et al., 2013; Reynaud et al., 2009; Wang and Douglas, 1999, 1998). 
Regardless of identity and origin, all mobile compounds must pass through the plasma membrane. Small uncharged molecules (e.g. $\mathrm{CO}_{2}, \mathrm{O}_{2}$ or $\mathrm{NH}_{3}$ ) permeate the membranes while most other molecules are transported either passively by channel proteins or actively by transporters or pumps (e.g. ATPases) (Lodish et al., 2016). Moreover, the endosymbionts are located in a host-derived late arrested phagosome, along with shed algal plasma membrane and thecal vesicles, forming the 'symbiosome membrane complex', which acts as an interface between the partner organisms (Fitt and Trench, 1983; Hohman et al., 1982; Neckelmann and Muscatine, 1983; Wakefield et al., 2000; Wakefield and Kempf, 2001). Therefore, exchanged nutrients and metabolites must also pass across the symbiosome membrane, the algal plasma membrane, and in many instances, the chloroplast envelope. The levels of some intrinsic transporter proteins, such as those for ammonium and sugar have been previously reported to be upregulated in symbiosis, suggesting their significance in a functional, integrated symbiosis (Baumgarten et al., 2015; Lehnert et al., 2014; Oakley et al., 2016; Peng et al., 2010). However, our current knowledge about transporter proteins that regulate nutrient/metabolite trafficking into the symbiosome is very limited, although a few relatively recent studies, using bioinformatics or immunocytochemistry techniques, have identified putative symbiosomespecific transporters, including those for ammonium, glucose and sterols, and ion transporters (Barott et al., 2015b, 2015a; Dani et al., 2017; Sproles et al., 2018).

In the current study, the anemone Exaiptasia pallida (Grajales and Rodríguez, 2014; Weis et al., 2008), commonly known as 'Aiptasia', was used to localize and quantify the levels of specific transporters observed when the anemone associates with its native symbiont (Breviolum minutum) and two non-native (i.e. non-native) Symbiodiniaceae species (Durusdinium trenchii and Symbiodinium microadriaticum). Four different transporters were investigated using immunohistochemical techniques: ammonium transporter 1 (AMT1, KXJ28516); V-type proton ATPase (VHA, KXJ14631); facilitated glucose transporter member 8 (GLUT8, KXJ23301); and aquaporin-3 (AQP3, KXJ09068). Sugar transporters GLUT8 and AQP3 have been previously predicted via bioinformatics to be localized to the symbiosome membrane (Sproles et al., 2018), while VHA and AMT1 are upregulated at the protein level in the symbiotic state (Oakley et al., 2016; Sproles et al., 2019). Moreover, VHA has been immunolocalized to the symbiosome surface in the corals Acropora yongei and Stylophora pistillata (Barott et al., 2015b). Here, I localized candidate transporters with respect to tissue 
layer (i.e. epi- and gastrodermis) and proximity to the symbiosome, and used a semiquantitative approach to establish the influence of symbiotic state and symbiont identity on transporter expression.

The influence of symbiont identity on symbiosis function is an important consideration given the taxonomic and physiological diversity within the family Symbiodiniaceae (LaJeunesse et $a l ., 2018$ ) and the findings that corals can respond to changing temperatures by 'shuffling' or 'switching' their dinoflagellate endosymbionts in favour of more thermally-resistant types (Buddemeier and Fautin, 1993; Berkelmans and van Oppen, 2006; Cunning, Silverstein and Baker, 2015; Matthews et al., 2017; but see Gabay et al., 2019). Durusdinium trenchii is especially interesting in this regard, given its reputation as an opportunistic species that may colonize corals post-bleaching, but which may provide the host with less nutritional advantage than the native symbiont and even induce cellular stress (Matthews et al., 2018, 2017; Pettay et al., 2015; Silverstein et al., 2017; Sproles et al., 2019). Given this, I hypothesized that the expression of the selected transporters would be downregulated in the presence of non-native $v s$. native symbiont species.

\subsection{Materials and Methods}

\subsubsection{Experimental organisms}

Three Symbiodiniaceae species - the native Breviolum minutum, and non-native Durusdinium trenchii and Symbiodinium microadriaticum - were grown at $25^{\circ} \mathrm{C}$ and $100 \mu \mathrm{mol}$ photons $\mathrm{m}^{-2}$ $\mathrm{s}^{-1}$ irradiance on a $12 \mathrm{~h}: 12 \mathrm{~h}$ light:dark cycle in $\mathrm{f} / 2$ medium in seawater passed through a 0.22 $\mu \mathrm{m}$ filter (FSW) for eight weeks prior to using the cultures for the experiments. B. minutum (culture ID FLAp2) was originally isolated from Aiptasia, while S. microadriaticum (CCMP2467) and D. trenchii (Ap2) were isolated from the coral Stylophora pistillata and an unknown anemone in Okinawa (Japan), respectively. To confirm the identity of these cultures, DNA was extracted using CTAB/phenol-chloroform (Baker et al., 2004), and the internal transcribed spacer (ITS2) region of ribosomal DNA amplified by polymerase chain reaction using ITSintfor 2 and ITS2Rev2 as the forward and reverse primers, respectively (LaJeunesse and Thornhill, 2011; Thornhill et al., 2007). Amplicons were directly sequenced (Macrogen 
Inc., Seoul, South Korea), sequences aligned with Geneious Prime v. 2019.2.3 (Biomatters Ltd., Auckland, NZ), and a custom BLAST search carried out against Symbiodiniaceae ITS2 sequences in Geosymbio (Franklin et al., 2012).

Specimens of Aiptasia ( $\mathrm{n} \sim 400$ ) were taken from a clonal laboratory stock (strain ID: NZ1; originally from the Indo-Pacific region) and rendered aposymbiotic (i.e. symbiont-free) by incubating them in $0.19 \mathrm{mM}$ menthol with $5 \mu \mathrm{M}$ 3-(3,4-dichlorophenyl)-1,1-dimethylurea (DCMU) as the photosynthetic inhibitor for four weeks (Matthews et al., 2016). This absence of symbionts was confirmed via a lack of chlorophyll autofluorescence, assessed by confocal microscopy (100× magnification, Olympus Provis AX70).

\subsubsection{Inoculation of Aiptasia with symbiotic algae}

Aposymbiotic anemones were starved for one week prior to inoculation with algal cultures. One drop of symbiont cells at $\sim 3 \times 10^{6}$ cells $\mathrm{mL}^{-1}$, mixed with a dilute suspension of Artemia sp. nauplii to induce phagocytosis, was pipetted onto each anemone's oral disc $(\mathrm{n} \sim 100$ anemones per symbiont species). Anemones were incubated in this symbiont suspension for $24 \mathrm{~h}$ before the FSW was changed. All anemones were maintained for eight weeks at $25^{\circ} \mathrm{C}$ under $100 \mu \mathrm{mol}$ photons $\mathrm{m}^{-2} \mathrm{~s}^{-1}$ irradiance on a $12 \mathrm{~h}: 12 \mathrm{~h}$ light:dark cycle and then flash-frozen in liquid nitrogen or used for immediate downstream analysis. Symbiont genetic identity was confirmed again after the experiment, using the method described above.

\subsubsection{Polyclonal antibody design}

Sequences of previously identified potential transporters (Lehnert et al., 2014; Oakley et al., 2016; Sproles et al., 2018) and homologous proteins from other model organisms, were used to search the available Aiptasia genome (Baumgarten et al., 2015) and transcriptome (Lehnert et al., 2014, 2012) using BLAST at an E-value of $1 \times 10^{-10}$. Highly similar sequences $(>40 \%)$ were selected as candidates (Geneious Prime v2019.2.3, Biomatters Ltd). 
Antigenic regions of each candidate transporter were predicted by EMBOSS (Rice et al., 2011), and epitopes were selected based on a set of criteria that included conservation level, antigenicity and whether the epitopes were external to the lipid bilayer (Fig. S4.1). In addition, the similarity of each selected epitope was compared to the protein sequences encoded on the genome of the animal used to generate the antibodies (Oryctolagus cuniculus, European rabbit) and on the Aiptasia and genomes/transcriptomes (Baumgarten et al., 2015; Lehnert et al., 2014; Parkinson et al., 2016), to maintain high antigenicity and limit cross-reactivity.

Custom-made rabbit polyclonal antibodies against AMT1, GLUT8 and AQP3 were developed using synthetic peptide antigens for the candidate transporters (Table S4.1). The rabbits were immunized with $200 \mu \mathrm{g}$ KLH conjugated peptide antigens. Primary immunizations were followed by three boosts of $200 \mu \mathrm{g}$ antigen every two weeks. Antibody titres were evaluated by ELISA 63 days following the initial injection. An affinity column with $5 \mathrm{mg}$ peptide antigen was used for antibody purification (Genscript USA, Inc.). Pre-made polyclonal rabbit antiVHA Subunit B was obtained from Genscript (No. A00960) because of the selected regions of the protein used for antibody generation were high similar to regions of the Aiptasia homolog (Fig. S4.1).

\subsubsection{Protein extraction}

Aposymbiotic and symbiotic anemones were rapidly frozen and those of the same type were pooled ( $\mathrm{n} \sim 5$ per pool). Total proteins of aposymbiotic anemones were extracted by homogenizing the samples in protein extraction buffer (100 mM Tris, $100 \mathrm{mM} \mathrm{NaCl}, 10 \mathrm{mM}$ EDTA, 5\% SDS, pH 7.4) using a tissue homogenizer at $4{ }^{\circ} \mathrm{C}$, and then denaturing the proteins at $80{ }^{\circ} \mathrm{C}$ for $15 \mathrm{~min}$ in the presence of $5 \% \beta$-mercaptoethanol. The homogenate was centrifuged at $20,238 \times g$ for $10 \mathrm{~min}$ and the supernatant decanted and used for downstream analysis. Symbiotic anemones were mechanically homogenized in $500 \mu \mathrm{FSW}$, and the resulting homogenate centrifuged at $500 \times g$ for 5 min to separate host (supernatant) and symbiont (pellet) fractions; total protein of the symbiotic host fraction was extracted as described above. Symbiont pellets were resuspended in $500 \mu \mathrm{FSW}$ and then passed through a 23 -gauge needle ( $0.337 \mathrm{~mm}$ inner diameter) with a $2.5 \mathrm{ml}$ syringe. Symbiont suspensions were centrifuged again at $500 \times \mathrm{g}$ for $5 \mathrm{~min}$ to remove host contamination and finally resuspended in protein extraction 
buffer. Algal resuspensions were ultrasonicated for $1 \mathrm{~min}$ to break the thecal plates, before being submitted to the aforementioned extraction method. Protein concentrations were assayed with a Qubit ${ }^{\circledR}$ 2.0 Fluorometer using a Protein Assay Kit (ThermoFisher Scientific).

\subsubsection{Immunoblotting}

Expression of all candidate transporters was confirmed via immunoblots with antibodies against AMT1, VHAв, GLUT8 and AQP3. Equal concentrations of total extracted proteins from each sample, including symbiotic host fraction, aposymbiotic host, and symbionts, were loaded and resolved by Tris-Glycine SDS PAGE. The gel concentration was 10\% polyacrylamide for AMT1, GLUT8 and $\mathrm{VHA}_{\mathrm{B}}$, and 15\% polyacrylamide for AQP3. A prestained molecular weight standard (PageRuler ${ }^{\mathrm{TM}}$ Prestained Protein Ladder, ThermoFisher Scientific) was used as a loading control for each gel. Resolved proteins were transferred to $0.45 \mu \mathrm{m}$ PVDF membranes overnight using Tris-Glycine-Methanol Transfer buffer (Towbin and Gordon, 1984) in a wet blotting system (Mini-PROTEAN Tetra Cell, BioRad). PVDF membranes were washed three times for 5 min with Tris-buffered saline containing $0.1 \%$ TWEEN 20 (TBST) and then blocked with fresh TBST buffer and 5\% fat-free milk at room temperature for $1 \mathrm{~h}$. Membranes were incubated with custom-made polyclonal primary antibodies overnight at $4{ }^{\circ} \mathrm{C}$ with agitation (see Table S4.1 for specifics of each antibody incubation). Membranes were then washed three times with TBST and then incubated with the secondary antibody (1:2000, Goat Anti-Rabbit IgG conjugated with AlexaFluor ${ }^{\circledR 555}$, ab150078 Abcam) for $2 \mathrm{~h}$ in the dark at room temperature with agitation. The membranes were then washed three times with TBST, and the immunoreactive proteins visualized by a Typhoon $^{\mathrm{TM}}$ FLA 9500 laser scanner (GE Healthcare Life Sciences). As a control, the procedure was performed as described above but without inclusion of the primary antibodies. The specificity of all primary antibodies was checked and validated by peptide pre-adsorption controls, incubating them with double the concentration of peptide antigen in TBST 5\% milk powder buffer at room temperature for $2 \mathrm{~h}$. The pre-immune serum and pre-adsorbed primary antibodies were used for immunodetection; neither the pre-adsorbed primary antibodies nor secondary antibodies reacted on immunoblots. Moreover, antibodies in the pre-immune sera reacted with 'nonspecific' proteins of different molecular weights (Fig. S4.2). 


\subsubsection{Immunolocalization}

Whole symbiotic anemones harbouring different Symbiodiniaceae species and aposymbiotic anemones were fixed in freshly made $4 \%$ paraformaldehyde in FSW at $4{ }^{\circ} \mathrm{C}$ overnight. The whole, fixed anemones were fully submerged in 10\%, 20\%, and $30 \%$ sucrose as a cryoprotectant at $4{ }^{\circ} \mathrm{C}$, embedded with OCT medium and rapidly frozen in liquid nitrogen, and the tentacles sectioned $(12 \mu \mathrm{m})$ at $-20{ }^{\circ} \mathrm{C}$ (CM3050 cryostat, Leica Biosystems). The cryosections were mounted on positively-charged slides and permeabilized with PBS buffer containing $0.1 \%$ Triton $\mathrm{X}-100$ in a humidified bath, followed by three washes PBS- $0.1 \%$ TWEEN. Sections were immersed in PBS- $0.1 \%$ Tween containing 10\% normal goat serum and $1 \% \mathrm{BSA}$ and maintained for $2 \mathrm{~h}$ at room temperature. Sections were then incubated with different concentrations of primary antibodies in blocking buffer at $4{ }^{\circ} \mathrm{C}$ overnight (see Table S4.1), followed by incubation with secondary antibodies (1:500) for $2 \mathrm{~h}$. Mounting medium containing $2 \mu \mathrm{g} / \mathrm{ml}$ DAPI, for DNA staining, was applied on sections before sealing with a coverslip. In the controls, sections were incubated with the pre-adsorbed primary antibody, as described above, except that the PBS-01\% Tween buffer was used instead of TBST. Aposymbiotic and symbiotic controls were also included. As for the immunoblots, peptide antigen pre-adsorption and secondary antibody controls did not show any binding reaction with the cryosections (Fig. S4.3). To assess differential expression of each transporter among the different host tissue layers, cryosections were treated identically (i.e., primary antibody concentrations and incubation time).

Immunolabelled sections were visualized by confocal laser scanning microscopy (Fluoview FV3000, Olympus, Japan) at 100× magnification. A combination of DAPI, AlexaFluor555 and AlexaFluor647 was used for fluorescence visualization of the DNA/nucleus (430 nm), the location of the various proteins that react with the primary antibodies $(570 \mathrm{~nm})$, and chlorophyll autofluorescence $(650 \mathrm{~nm})$. A total of $261 \mathrm{z}$-stacks (nominal thickness $>10 \mu \mathrm{m}$ ) were analysed for semi-quantitative comparisons of transporter abundance and localization within the host's tissue layers. I analysed two tentacles in each of ten anemones, for locating each of the different transporters (Table S4.2). All z-stacks were acquired with the same settings (e.g., laser intensity, sensitivity, gain, offset and exposure-time) (Arena et al., 2017; Jonkman et al., 2020; Kelly and Hawken, 2017; Miura and Sladoje, 2019; Pike et al., 2017). 


\subsubsection{Semi-Quantitative confocal analysis}

An analysis pipeline was developed to quantify the relative amount of AlexaFluor555 intensity in each host tissue layer, as well as host tissue volume and the number of symbiont cells in each z-stack, using FIJI (ImageJ2) (Rueden et al., 2017; Schindelin et al., 2012). All z-stacks were split into different channels, and 3-D gaussian blur and background subtraction methods were used to smooth the images and remove noise on all channels prior to downstream analyses (Kelley and Paschal, 2019; Tivey et al., 2020). The AlexaFluor555 channel was manually segmented to acquire images from host gastrodermis. Total volume and signal intensities were quantified in segmented and unsegmented AlexaFluor555 gastrodermis images using 3D Object Counter Plugin (Bolte and Cordelières, 2006). Volumes and signal intensities of epidermal objects were calculated by subtracting the total of the gastrodermal values from the unsegmented AlexaFluor555 channel. Symbiont cells were defined as regions-of-interest (ROIs) by creating a mask via thresholding intensities of the AlexaFluor647 channel, corresponding to chlorophyll autofluorescence. Overlapping symbiont cells were separated by applying a round of 3D watershed split (Tivey et al., 2020). All symbiont objects were counted by the same plugin. Host tentacle volume for each z-stack was quantified as described above, using the brightfield channel.

AlexaFluor555 intensity and the number of symbiont cells were each normalized to the host tissue volume. Data normality was checked by the Shapiro-Wilk test and a mixed two-way analysis of variance (ANOVA) was used to compare differential expression of transporters in the different host tissue layers in both aposymbiotic and symbiotic anemones. This was followed by a Bonferroni post hoc test to determine if significant differences occurred in the various pairwise comparisons. Correlations between native symbiont density and transporter signal intensity of epidermis and gastrodermis were investigated using the Spearman correlation coefficient. All data analyses were conducted in R 4.0.3 (R Core Team, 2020). Zstack image analysis pipelines, FIJI scripts, data and supplementary files are available at GitHub (https://github.com/Amir-Mashini/Aiptasia-Transporters-Quantification-Project). 


\subsection{Results}

\subsubsection{Colonization success}

No symbionts were detected by confocal examination of aposymbiotic anemones before colonization or in control aposymbiotic anemones at the end of the experiment (Fig. S4.4). Direct ITS2 sequencing confirmed the genetic identities of symbionts in the colonized anemones. Symbiont densities were approximately three-fold greater (one-way ANOVA, $p=$ $2.2 \times 10^{-7}$ ) in anemones hosting $B$. minutum than those recolonized by $D$. trenchii or $S$. microadriaticum by the end of the experiment (Fig. 4.1), which is consistent with previous studies (Gabay et al., 2018; Sproles et al., 2019).

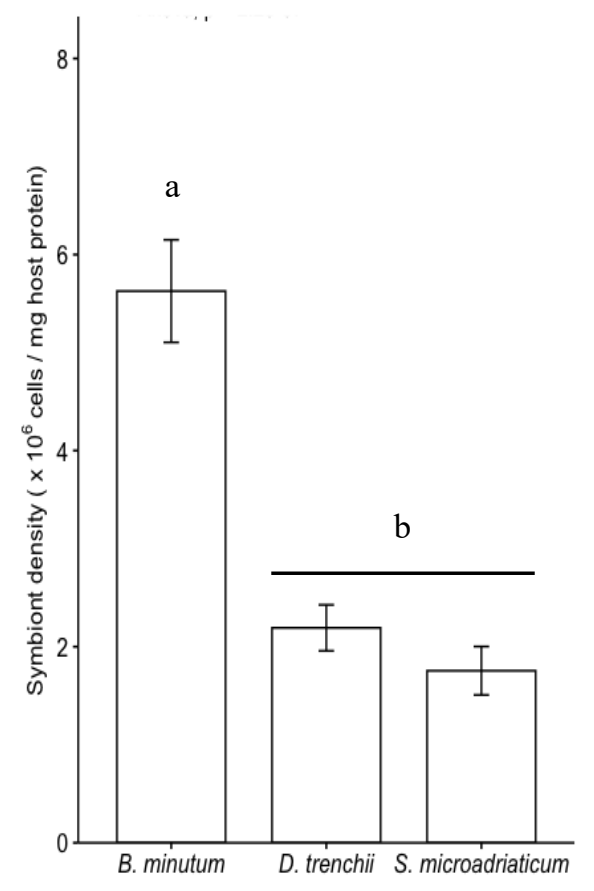

Figure 4.1. Colonization densities of different symbiont species (Breviolum minutum, Durusdinium trenchii, Symbiodinium microadriaticum) in Aiptasia, eight weeks postinoculation. Different letters above bars indicate significant differences between treatments $(p<0.05)$. Values are mean \pm $\mathrm{SE}, \mathrm{n}=24$.

4.3.2 Localization of transporters in the native symbiosis

Symbiosis with the native B. minutum induced distinct changes in the accumulation of the various nutrient transporters when compared to the aposymbiotic state, in both the gastrodermis and epidermis (Fig. S4.2); these patterns are identical to those observed with 
immunolocalization (Fig. 4.2). Nevertheless, symbiont cell density did not correlate with expression of any of the transporters in either tissue layer (Fig. S4.5; $p>0.05$ ). AMT1 and VHA were exclusively expressed in the symbiotic state, although they exhibited dissimilar expression patterns. Specifically, AMT1 was 1.7-fold more highly expressed in the epidermis than the gastrodermis (Two-way mixed ANOVA, $p=1.03 \times 10^{-6}$; Fig. 4.3), while VHA expression was 3.1-fold greater in the gastrodermis than the epidermis $\left(p=4.85 \times 10^{-7}\right)$. In contrast, GLUT8 and AQP3 were expressed in both the aposymbiotic and symbiotic states, though their patterns of expression were dissimilar. Specifically, GLUT8 expression was the same in both aposymbiotic and symbiotic anemones, with an even distribution in both host tissue layers, while AQP3 was 4.7-fold more highly expressed in the aposymbiotic state ( $p=$ $3.27 \times 10^{-27}$ ), and 1.5 -fold more highly expressed in the epidermis than the gastrodermis with B. minutum.

The cellular localization of the transporters was further resolved through examination of host cells containing B. minutum (Fig. 4.4; supplementary videos 1-4). The transporters were located at the perimeter of symbionts, the host gastrodermal membrane, or both, except for VHA which was only found at the perimeter of the symbionts, and AQP3 which was only detected at the host cell membrane.

\subsubsection{Effects of symbiont identity on transporter localization}

The presence of non-native symbionts affected transporter localization (Fig. 4.3). Unlike in the native symbiosis, D. trenchii-colonized anemones had AMT1 distributed evenly across both tissue layers, although the fluorescence intensity was reduced by about a half in the epidermis relative to $B$. minutum-colonized anemones $\left(p=4.89 \times 10^{-6}\right)$. VHA in D. trenchii-colonized anemones exhibited a localization pattern similar to that in B. minutum-colonized anemones, being expressed 2.1-fold more highly in the gastrodermis than epidermis $\left(p=5.18 \times 10^{-3}\right)$, though this gastrodermal expression was weaker than in the native symbiosis $\left(p=1.16 \times 10^{-3}\right)$; epidermal expression was similar between the two symbioses. In contrast, GLUT8 expression in D. trenchii-colonized anemones was similar across the gastrodermis and epidermis, and similar in both intensity and pattern to anemones populated by B. minutum. AQP3 was likewise expressed at similar levels in the epidermis and gastrodermis of $D$. trenchii-colonized 
anemones, though in both tissue layers this transporter was more highly expressed (two-fold) than in the native symbiosis (epidermis: $p=1.56 \times 10^{-3}$; gastrodermis: $p=1.31 \times 10^{-7}$ ).

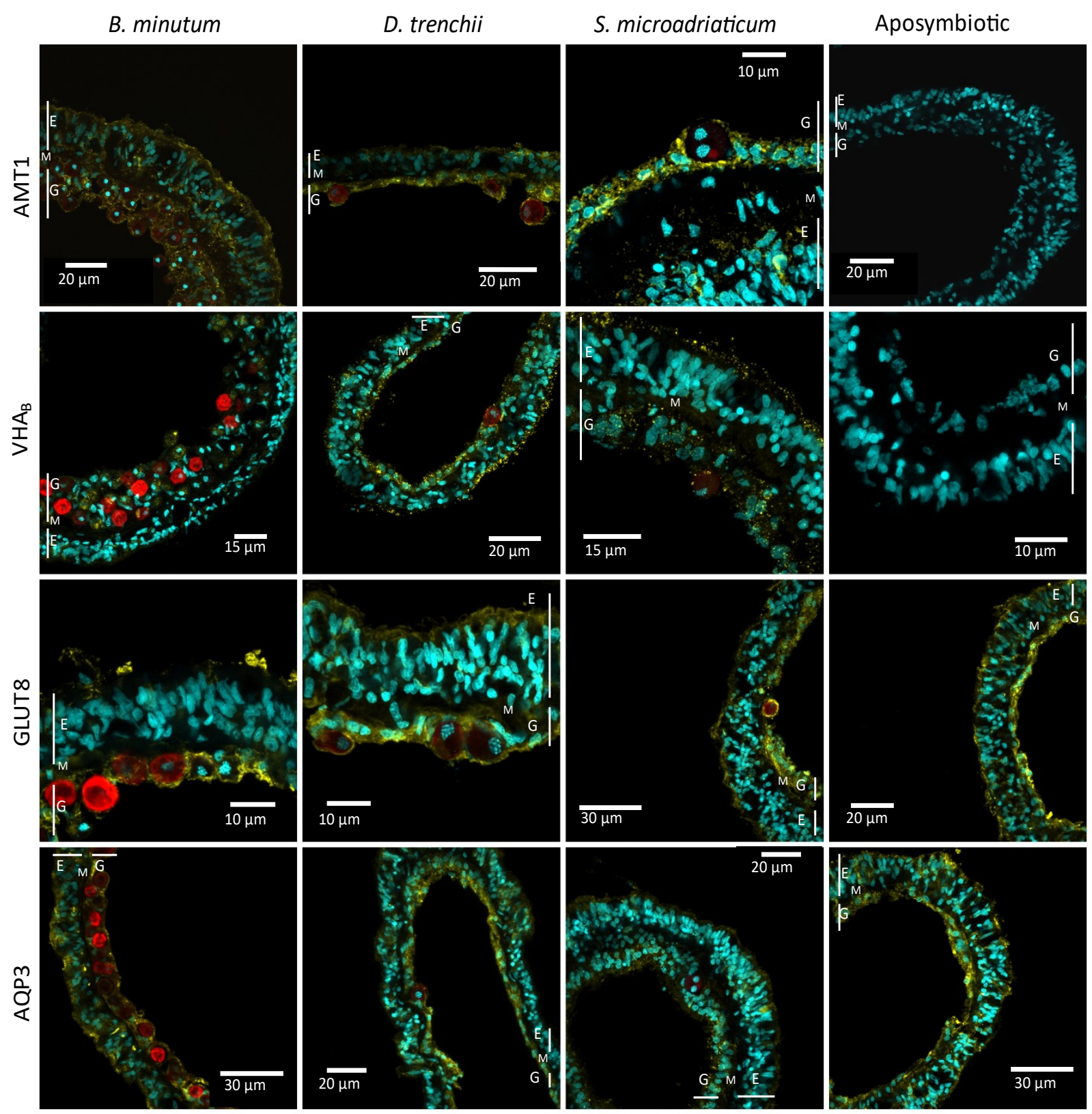

Figure 4.2. Immunofluorescence localization of nutrient transporters in aposymbiotic and symbiotic Aiptasia hosting Breviolum minutum, Durusdinium trenchii and Symbiodinium microadriaticum. Aposymbiotic anemones showed no visible expression of AMT1 or $\mathrm{VHA}_{\mathrm{B}}$. Red, chlorophyll autofluorescence of symbionts; cyan, nuclear staining of host and symbionts using DAPI; yellow, anti-AMT1, anti-VHAB, anti-GLUT8 and anti-AQP3 localizations using AlexaFluor555 conjugated secondary antibody. 

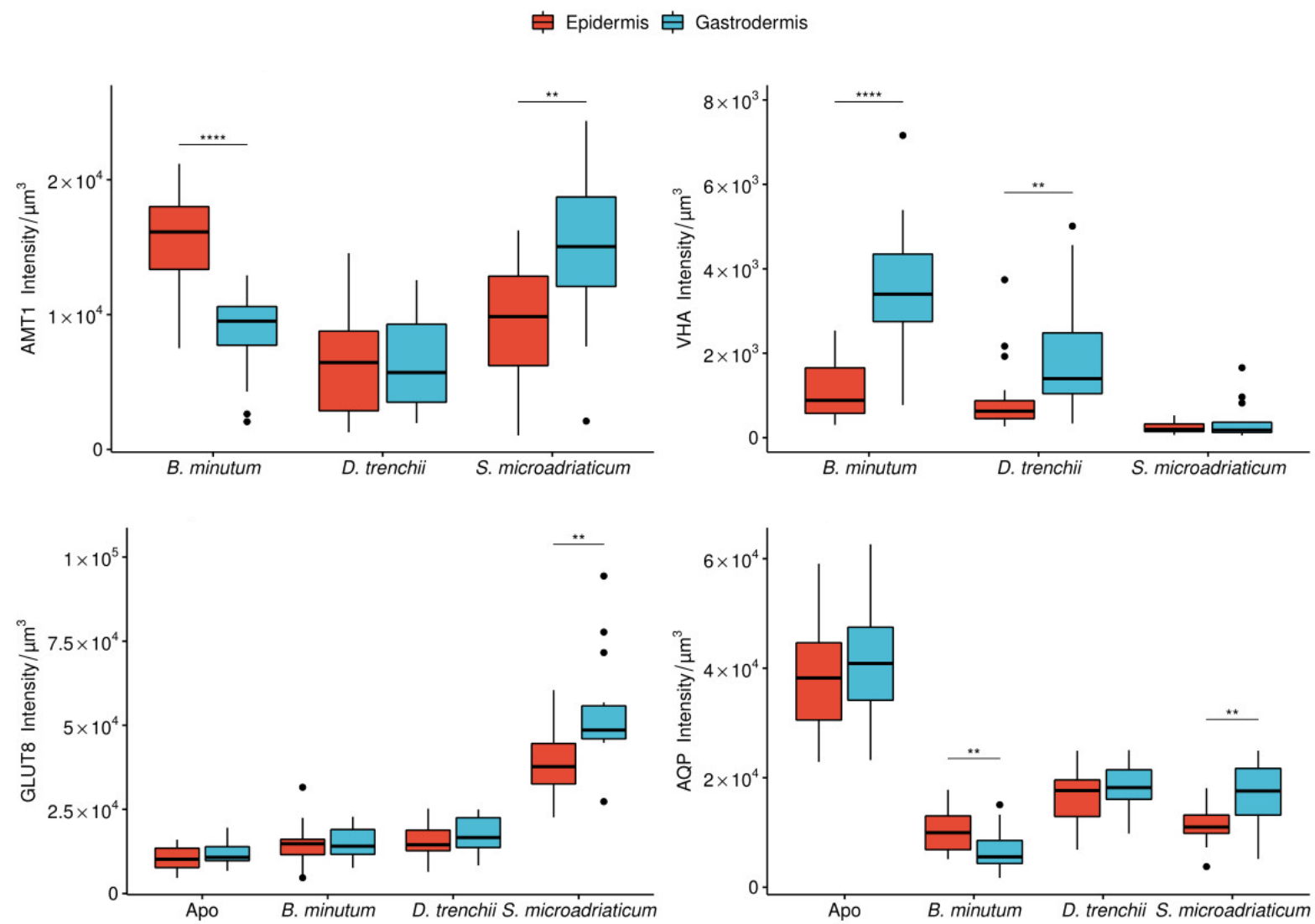

Figure 4.3. Signal intensities of quantified transporters in Aiptasia tentacles in different symbiotic states. Two-way mixed ANOVA was performed to compare intensity level between symbiotic states and tissue layers. Asterisks indicate significant differences between tissue layers of each treatment $\left({ }^{* *} \mathrm{p}<0.01\right.$ and $\left.{ }^{* * * *} \mathrm{p}<0.0001\right)$.

S. microadriaticum-colonized anemones showed the most distinct expression patterns from those in the native symbiosis. For example, AMT1 was more highly expressed (1.5-fold) in the gastrodermis rather than the epidermis $\left(p=1.39 \times 10^{-3}\right)$. This difference resulted from a $36 \%$ decrease in AMT1 expression in the epidermis and a 1.6-fold increase in the gastrodermis of $S$. microadriaticum-colonized anemones relative to the native symbiosis $\left(p=2 \times 10^{-3}\right.$ and $p=$ $2.22 \times 10^{-4}$, respectively). Unlike in B. minutum-colonized anemones, anemones harbouring $S$. microadriaticum exhibited an even distribution of VHA across both tissue layers, though the intensity was about seven times less than in the presence of the native symbionts (epidermis $p$ $=1.16 \times 10^{-3}$; gastrodermis $p=2.22 \times 10^{-8}$ ). Furthermore, in contrast to the distribution seen with $B$. minutum (and indeed D. trenchii), GLUT8 and AQP3 were relatively more abundant in the gastrodermis than epidermis of $S$. microadriaticum-colonized anemones $(\sim 1.5$-fold; $p=$ $4.49 \times 10^{-3}$ and $p=3.5 \times 10^{-3}$, respectively). When compared to the native symbiosis, GLUT8 expression in both tissue layers of $S$. microadriaticum-colonized anemones was about 1.7-fold 
higher (epidermis: $p=6.22 \times 10^{-16}$; gastrodermis: $p=9.65 \times 10^{-21}$ ), while AQP3 expression was significantly higher in the gastrodermis only $\left(2.6\right.$-fold; $\left.p=4.75 \times 10^{-5}\right)$.
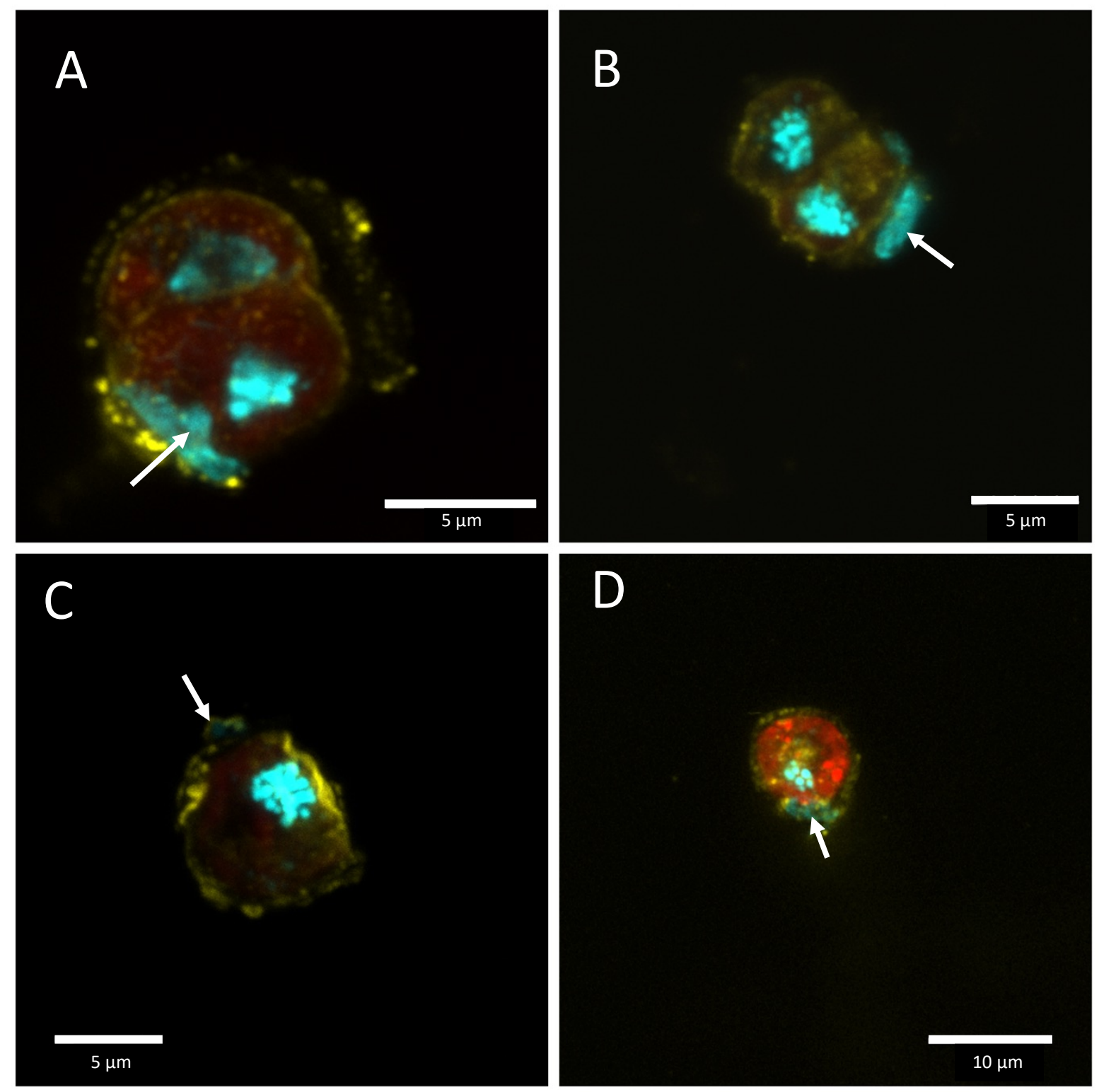

Figure 4.4. Intracellular localization of (A) AMT1, (B) VHA, (C) GLUT8 and (D) AQP3 on Aiptasia cells when hosting the native symbiont Breviolum minutum. Red, chlorophyll autofluorescence of symbionts; yellow, anti-AMT1, anti-VHA, anti-GLUT8 and anti-AQP3 localization using AlexaFluor555 conjugated secondary antibody; cyan, nuclear staining of host and symbionts using DAPI, permanently condensed chromosomes of symbionts are seen as patches of small blue round objects, host nucleus (white arrow) is usually compressed and located next to symbionts. 


\subsection{Discussion}

This study is the first to report the intracellular localization of AMT1, GLUT8 and AQP3, and their relative quantities in the tissue layers of a cnidarian-dinoflagellate model organism. Previously, only the sterol transporters NPC1 and NPC2, and VHA have been immunolocalized in symbiotic cnidarians (Barott et al., 2015b; Dani et al., 2017). Each transporter studied exhibited a distinct pattern of expression and localization in response to symbiotic state and/or symbiont identity. Of note, AMT1, VHA and GLUT8 were particularly abundant in close proximity to the symbiosome membrane complex, suggesting that they play a crucial role in symbiosis function.

\subsubsection{Ammonium transport}

Nitrogen fluxes are crucial for corals in oligotrophic tropical waters (Grover et al., 2008; Miller and Yellowlees, 1989; Rädecker et al., 2017b). In the cnidarian-dinoflagellate symbiosis, the symbionts are the primary site of ammonium assimilation, however it has been suggested that both partners can take up this nutrient, including from the surrounding seawater (Bednarz et al., 2020; Cui et al., 2018; Dudler and Miller, 1988; Grover et al., 2002; Lipschultz and Cook, 2002). Consistent with this, in the current study AMT1 was localized to both host epidermis and gastrodermis, though it was only expressed in the symbiotic state. Moreover, it was most intensely expressed in close proximity to the symbionts. Similarly, AMT1 has previously been found to be upregulated during symbiosis in Aiptasia, in both the transcriptome (Lehnert et al., 2014; Maor-Landaw et al., 2020) and proteome (Oakley et al., 2016), and be downregulated after heat shock and bleaching (Cleves et al., 2020).

Host epidermal cells directly take up ammonium from the ambient seawater (Pernice et al., 2012). Given that photosynthesis drives ammonium uptake (Falkowski et al., 1993; HoeghGuldberg, 1994; Xiang et al., 2020), photosynthetic rate per symbiont cell and symbiont population density both likely play an important role, providing a link between AMT1 expression and symbiont physiology. Consistent with this, B. minutum-colonized hosts exhibited greater expression of AMT1 in their epidermis than anemones colonized by either of the two non-native symbionts. Of note, D. trenchii and S. microadriaticum have previously 
been reported to be relatively unproductive partners compared to B. minutum when experimentally introduced into Aiptasia, while D. trenchii is widely acknowledged as an opportunistic species (Berkelmans and van Oppen, 2006; Matthews et al., 2017; Gabay et al., 2019, 2018; Sproles et al., 2019; Stat and Gates, 2010). Once assimilated, however, the host actively regulates nitrogen supply to the symbionts through the glutamine synthetase (GS) and glutamine 2-oxoglutarate amino-transferase (GOGAT) pathways, thereby maintaining a low ammonium concentration in the gastrodermal cytoplasm (Wang and Douglas, 1999, 1998; Xiang et al., 2020). This process again requires photosynthesis, with the provision of carbon compounds to the host being important for ammonium assimilation; conversely, aposymbiotic hosts excrete surplus ammonium to the surrounding seawater (Zamer and Shick, 1987). This might explain the opposite pattern of AMT1 expression seen in the gastrodermis vs. the epidermis, with $S$. microadriaticum-colonized anemones exhibiting a greater intensity of gastrodermal AMT1 than either the B. minutum- or D. trenchii-colonized anemones. In particular, S. microadriaticum has been reported to have a significantly lower rate of cellspecific photosynthesis than the other two species (Gabay et al., 2018), and was present at the lowest population density (though not significantly lower than $D$. trenchii) in the current study. It might therefore be expected that $S$. microadriaticum-colonized anemones contained the biggest pools of excretory ammonium in their tissues and hence the greatest need for AMT1. This might appear inconsistent with the apparent absence of AMT1 in aposymbiotic anemones, though my data suggest that the onset of symbiosis with any partner, however beneficial, is sufficient to trigger the expression of this transporter in the host. More detailed physiological examination of ammonium uptake and metabolism in the presence of different symbiont species is needed though, to more fully understand the transporter localization patterns observed here.

\subsubsection{Proton pumps and acidification}

Proton transporters are central to $\mathrm{pH}$ homeostasis in corals (Capasso et al., 2021). VHA, a member of the P-type ATPase superfamily, is found in all domains of life (Chan et al., 2010). VHA is primarily found in the vacuolar membrane as well as the Golgi complex, endosomes and synaptic vesicles, where it acidifies these organelles by hydrolysis of ATP and transport of protons across the membrane (Barott et al., 2015b; Graham et al., 2003). Similar to the findings of the current study, a proteomic survey also reported significantly increased expression (1288 
fold) of VHA in symbiotic vs. aposymbiotic Aiptasia (Oakley et al., 2016). In corals, VHA is abundant in the oral gastrodermis, specifically in the space immediately surrounding the endosymbionts where it plays a role in the host's carbon concentrating mechanism by acidifying the symbiosome space and facilitating photosynthesis (Barott et al., 2015b). Consistent with these previous observations, VHA in the current study was localized near the symbiosome membrane complex. In addition, VHA facilitates $\mathrm{CO}_{2}$ uptake by host epidermal cells, as exportation of protons decreases the $\mathrm{pH}$ of ambient seawater, increasing the dehydration of $\mathrm{HCO}_{3}{ }^{-}$to $\mathrm{CO}_{2}$ (Furla et al., 2000a). The localization of VHA to the outer surface of the epidermis here was, therefore, unsurprising.

VHA expression was influenced by symbiont identity, being more abundant in both the gastrodermis and epidermis when anemones were in symbiosis with $B$. minutum or D. trenchii than with S. microadriaticum. Proton ATPases have been reported in other mutualistic associations, where they are actively excluded by symbionts through different mechanisms in parasitic symbioses to prevent digestion by the host via acidification of phagosomes (Smith and Gianinazzi-Pearson, 1988; Sturgill-Koszycki et al., 1994; Vinet et al., 2009; Wong et al., 2011). However, whether the low expression of VHA in S. microadriaticum-colonized anemones is due to symbionts actively inhibiting host digestion or by the host reducing the symbiosome $\mathrm{CO}_{2}$ concentration to limit less beneficial symbionts is unknown.

\subsubsection{Carbon translocation}

In the current study, only the hosts harbouring non-native symbionts had significantly higher expression levels of GLUT8 compared to aposymbiotic animals, with S. microadriaticumcolonized anemones expressing GLUT8 more in the gastrodermis than the other anemones. Translocation of fixed carbon to the host has been researched intensively (Davy et al., 2012; Muscatine and Hand, 1958) and glucose has been proposed as the primary form of fixed carbon translocated, along with other hexoses and glycerol (Burriesci et al., 2012; Hillyer et al., 2017b; Muscatine, 1967; Suescún-Bolívar et al., 2016). SLC2 (GLUT) is a major facilitator superfamily of transmembrane transporters that mediates the transport of monosaccharides, polyols, and other small compounds across the membranes of eukaryotic cells (Augustin, 2010; Mueckler and Thorens, 2013). Specifically, GLUT8 is mostly expressed at endosomal 
compartments, however it can also be localized to the cell surface (DeBosch et al., 2014; Flessner and Moley, 2009; Ibberson et al., 2002, 2000). In cnidarians, GLUT8 had been hypothesized to have a primary role in glucose uptake by the host (Sproles et al., 2018) and molecular evidence has indicated its upregulation in symbiosis (Lehnert et al., 2014; Oakley et al., 2016). Why a non-native symbiont induced the greatest expression of GLUT8 is not clear, but it could be due to increased efforts by the host to translocate fixed carbon when in symbiosis with less nutritionally beneficial symbionts (Gabay et al., 2018). Indeed, this latter study showed that, when in symbiosis with Aiptasia, gross photosynthesis and the ratio of photosynthesis to respiration were similar in B. minutum- and D. trenchii-colonized anemones, but higher than in anemones containing $S$. microadriaticum.

Members of the aquaporin water channel family primarily render the lipid bilayer of cell membranes permeable to water, which is vital for cell osmoregulation and homeostasis (Campbell et al., 2008). Aquaglyceroporins, a subset of the AQP family, are also permeable to glycerol and specific small, uncharged solutes (Carbrey et al., 2003; Ishibashi et al., 2011). AQP3 channels were previously predicted to be localized to the outer symbiosome surface (Sproles et al., 2018), though here I only localized AQP3 to the host cell membrane. Aquaglyceroporins are involved in glycerol transport, and several reports have shown higher expression during fasting and downregulation after feeding in other organisms (Gambardella et al., 2012; Hibuse et al., 2006; Kuriyama et al., 2002; Liao et al., 2020; Maeda et al., 2004). Similarly, in corals, inhibition of photosynthesis by DCMU causes higher expression levels of AQP transcripts in host tissues, suggesting a potential cellular response to compensate for reduced availability of photosynthetic products (Yuyama et al., 2012). Consistent with this, I report greater expression of AQP3 in the aposymbiotic state, and enhanced AQP3 expression in the presence of the putatively less beneficial non-native $v s$. more beneficial native symbionts. Together, these observations provide strong evidence that AQP3 participates in glycerol transfer across the host membrane in the cnidarian-dinoflagellate symbiosis, though the transporters that facilitate glycerol trafficking across the host-symbiont interface are still unknown. 


\subsection{Conclusion}

My findings depict effects of symbiotic state and identity of symbionts on the quality and quantity of the candidate metabolite transporters. Obtaining novel symbionts has been proposed as a temporary strategy in response to different environmental stressors, such as increasing temperature (Cunning et al., 2015b; Reich et al., 2017). However, D. trenchii and S. microadriaticum have been previously observed to be less beneficial when inoculated into Aiptasia (Gabay et al., 2018; Matthews et al., 2018, 2017; Sproles et al., 2019), while D. trenchii is a well-known opportunist on coral reefs (Pettay et al., 2015; Stat and Gates, 2010). Consistent with this, my results suggest that anemones harbouring non-native symbionts show less metabolite transporter compatibility compared to anemones containing the native symbiont, at least with respect to the transporters studied here. Further research investigating these, and other, transmembrane transporters in depth, including their co-localization patterns, is needed both in model systems and reef corals to understand the mechanisms required for fully integrated metabolic exchange and symbiosis success. Ultimately, such studies will allow us to better understand and predict how coral reefs will respond to climate change and to better manage these changes. 


\section{Chapter 5 \\ General Discussion}

\subsection{Summary}

There are still major knowledge gaps in our understanding of fundamental cellular processes and nutritional exchange between symbiotic cnidarians and their photosynthetic endosymbionts. Importantly, this knowledge is crucial for understanding how corals might adapt to climate change. Thus, the aim of this thesis was to characterize the cellular mechanisms that are important in metabolic exchange and integration of the symbiotic partners, and enhance our understanding of the potential for reef corals to adapt to climate change via partner 'switching' or 'shuffling'.

Proteomics was used to decipher cellular pathways that are affected in both native and nonnative Symbiodiniaceae, when ex hospite or residing in a model symbiotic cnidarian (Exaiptasia pallida: 'Aiptasia') (Chapters 2 and 3). Analysis of the proteomes of the native symbiont, Breviolum minutum, identified cellular pathways involved DIC and DIN uptake that are actively upregulated when the symbiont is ex hospite (Chapters 2), while in hospite the symbionts are provided with DIC and DIN from the host (Chapter 4) (Fig. 5.1). Additionally, under nutrient-limited conditions (both in and ex hospite), the capacity for the symbionts to scavenge other nutrients, such as iron, was observed to increase, despite the photosynthetic capability of the symbionts declining (Chapter 2). These findings support the well-established view that the symbiosis is nutritionally beneficial to the symbionts, but that high symbiont population densities and rates of photosynthesis can risk oxidative stress, triggering the symbiont's oxidative stress response network (Chapters 2).

Another key finding was that changes in the symbiont proteome during colonization of the host mostly reflect the symbiont's need for light harvesting, photosynthesis, and chloroplast repair and maintenance (Chapter 3). Furthermore, additional changes were consistent with increasing nitrogen limitation over the course of host colonization, likely as the combined 
consequence of host control of nutrient supply, and the increasing symbiont cell density and hence competition for resources.

Both native and non-native Symbiodiniaceae were observed to suppress the host's immune response via a range of cellular pathways (Chapter 3). However, immunosuppression of the host by the native symbionts was more likely aimed at the avoidance of host autophagy or expulsion, while the non-native Durusdinium trenchii appeared to more directly manipulate host cell gene expression in a manner more akin to pathogenic apicomplexans and viruses (Fig 5.1) (Chapter 3). Immunocytochemical evidence is presented for the reduced capacity for nonnative symbionts to receive DIN and DIC from the host when compared to the native symbionts, while conversely, non-native symbionts provide the host with less photosynthetically-fixed carbon (Chapter 4) (Fig. 5.1). These finding build on previous studies (Gabay et al., 2019; Matthews et al., 2017; Sproles et al., 2019), supporting the opinion that the non-native $D$. trenchii is an opportunist, providing less nutritional support to a non-native host (in this case Aiptasia) while failing to establish a well-integrated, fully functional symbiosis.

My findings raise some important questions with respect to the mechanisms that underpin a functional symbiosis and determine the flexibility to establish novel host-symbiont pairings. Three questions are of particular interest and will be considered further here: (1) What is the potential for the evolution of novel cnidarian-dinoflagellate symbioses? (2) Does the degree of cellular integration between the symbiotic partners reflect the position of the symbiosis on the mutualism - parasitism continuum? And (3) what are the ecological implications for the survival of coral reefs in the face of climate change, and the development of tools for increasing reef resilience?

\subsection{Partner integration and symbiosis function}

5.2.1 What is the potential for the evolution of novel cnidarian-dinoflagellate symbioses?

Established symbiotic associations have the potential for coevolution based on interspecies interactions (Guerrero et al., 2013; Mason et al., 2017). Scleractinian corals have an evolutionary origin within the Paleozoic Era (more than 400 MYA); however, 93 
Symbiodiniaceae are relatively newer and are estimated to have evolved approximately 160 MYA, which coincides with the second adaptive radiation of scleractinian corals during the Middle Jurassic Period (Van Oppen and Medina, 2020). Thus, both partners have been subject to natural selection pressures across eras, resulting in the integration of the numerous interpartner cellular processes seen in contemporary cnidarian-dinoflagellate symbioses.

One of the most prominent of these integrated cellular processes is the uptake of dissolved inorganic carbon (DIC), which is facilitated via carbon concentrating mechanisms (CCMs). Host carbonic anhydrase (CA), being a central component of CCMs, converts bicarbonate from ambient seawater to carbon dioxide, which can be used by both symbionts and host in different cellular processes, such as photosynthesis and calcification (Furla et al., 2000b). Moreover, host VHA (vacuolar proton pump) works in harmony with CA to facilitate bicarbonate conversion to carbon dioxide; in particular, VHA is important with respect to the translocation of DIC to the symbionts for photosynthesis (Fig. 5.1) (Barott et al., 2015b; Furla et al., 2000a). These integrated pathways are likely to have evolved to support the DIC demand of both partners, however the presence of non-native symbionts likely interrupts the process, resulting in reduced delivery of DIC (Chapter 4). Moreover, the DIC delivered to the symbionts is primarily used for photosynthesis, with photosynthetically-fixed carbon being translocated to the host in numerous forms, the most abundant of which is now believed to be glucose (Burriesci et al., 2012; Hillyer et al., 2017b). This translocation is likely to be achieved by host sugar transporters such as AQP and GLUT8 (Sproles et al., 2018), or sterol transporters such as NPC2 (Dani et al., 2017; Sproles et al., 2019), but once again this inter-partner process can be hindered by reduced host-symbiont compatibility in a novel partnership (Chapters 3 and 4). There is therefore strong evidence that carbon metabolism and flux are less effective in new symbioses, due to poor host-symbiont cellular integration.

Nitrogen supply is also of paramount importance in nutrient-poor tropical seas, and hence the bi-directional flux of this nutrient is tightly regulated in the cnidarian-dinoflagellate symbiosis, with the symbionts being dependent on the host for DIN via its heterotrophic feeding and metabolic waste, and the host receiving amino acids (including essential amino acids) from the symbionts (Wang and Douglas, 1999) (Chapter 4). Ammonium is the major form in which nitrogen is absorbed by scleractinian corals and other cnidarians, and both symbionts and host can assimilate it (Pernice et al., 2012; Wang and Douglas, 1998). However, nitrate and nitrite, 94 
as well as dissolved organic nitrogen (DON) compounds such as urea and amino acids, can also be taken up by symbiotic cnidarians and their dinoflagellate partners (Grover et al., 2003, 2006; Kopp et al., 2013; Tanaka et al., 2006). Furthermore, cnidarian hosts control their symbiont population, in part, by regulating nitrogen supply (Wang and Douglas, 1999, 1998; Xiang et al., 2020). Consistent with this, in the current study, the proteomes of both B. minutum and $D$. trenchii demonstrated a downregulation of ammonium assimilation by the symbionts when in hospite (Chapters $\mathbf{2}$ and $\mathbf{3}$ ).

A key aspect of the cellular integration required for the effective movement of nitrogen between the partners is the host ammonium transporter protein (AMT). AMT transporters, which are found in all kingdoms of life, are integral transmembrane proteins and are vital for ammonium transportation (Andrade et al., 2005; Lamoureux et al., 2010). In the cnidariandinoflagellate symbiosis, a homologue of AMT is only expressed when the host is in a symbiotic relationship (Lehnert et al., 2014; Oakley et al., 2016; Sproles et al., 2019; Chapter 4), and, of note, is localized to the proximity of the symbiosome membrane complex (Chapter 4). However, in the current study it was found that the expression of AMT in the host's tissues was heavily affected when in symbiosis with the non-native, less beneficial (Gabay et al., 2018) S. microadriaticum, triggering higher expression levels of AMT in the host (Chapter 4).

In addition to nutritional interactions, a fully integrated symbiosis is dependent on the molecular communication between partners to ensure its persistence, however very little is still known about the signalling cascades involved (Rosset et al., 2021). Cellular signalling is typically comprised of primary and secondary messengers. Primary signalling molecules activate specific cell-surface receptors of target cells by binding to them, which triggers a signal transduction cascade that transfers the signal inside the cell (Lodish et al., 2016). The phosphatidylinositol signalling system (PtdIns) is one the secondary messenger pathways which, based on recent evidence, has an important role in the cnidarian-dinoflagellate symbiosis (Bellantuono et al., 2019; Hillyer et al., 2017a; Matthews et al., 2017). This pathway is manipulated in the host by certain bacterial pathogens and protozoan parasites to facilitate host cell invasions and intracellular persistence (Drecktrah et al., 2004; Ruhland et al., 2007). PtdIns and the inositol phosphate metabolism pathway (InsP), being vital for symbiosis, are 
also common in Symbiodiniaceae (Rosic et al., 2015). Likewise, in the current study, homologues of glycerophosphoinositol permease 1 (GIT1), which facilitates glycerophosphoinositol (GPI) transport across the cell membrane, were upregulated only in the native symbiont when in hospite (Chapters 2 and 3). These GPI anchors might be used as a means of communication and cell recognition between partners (Davy et al., 2012). Interestingly, a homologue of another protein which is likely part of PtdIns, phosphatidylinositol phosphatase (INP3), was exclusively found in the native symbiont in the current study when in symbiosis (Chapter 2; Fig. 5.1). The presence of PtdIns in the native symbiont when in hospite, and absence of it in the non-native symbionts, implies higher levels of integration between the partners as a result of coevolution. Thus, inositol pathways may be critical for the establishment and persistence of compatible symbioses; further investigation is essential to fully understand the role of PtdIns in the cnidarian-dinoflagellate symbiosis.

Taken together, these observations suggest sub-optimal cellular integration and symbiosis performance in novel symbioses when compared to the compatible, native symbiosis, which has major implications for the survival of cnidarians that change their usual, dominant symbiont type, for example after a bleaching event (Cunning et al., 2015a; Dilworth et al., 2021). This topic is discussed further in Section 5.3. 
In symbiosis

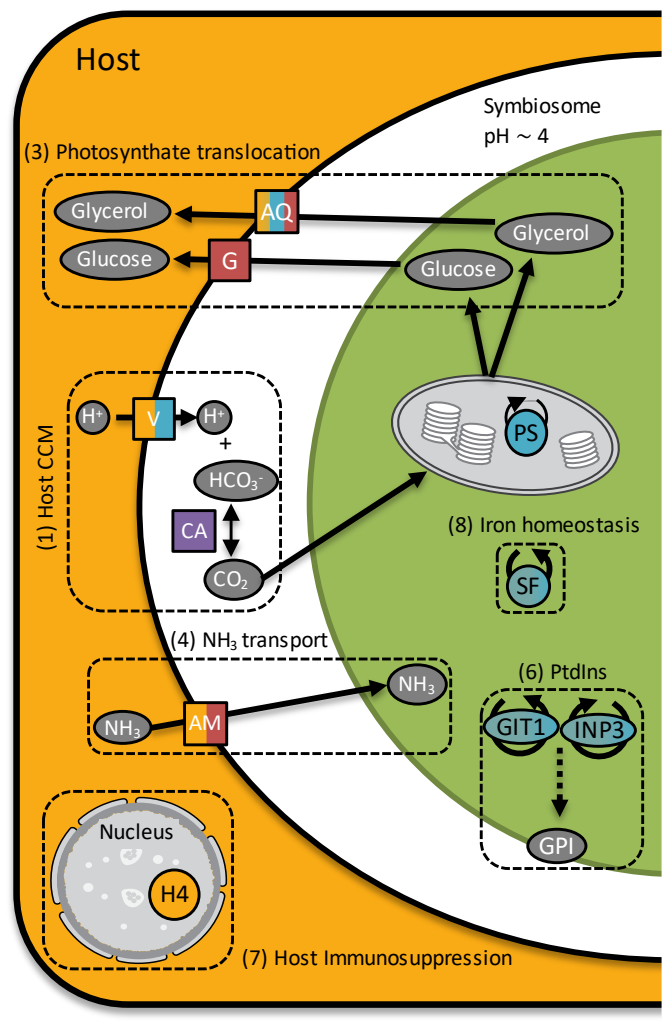

Free-living

Seawater

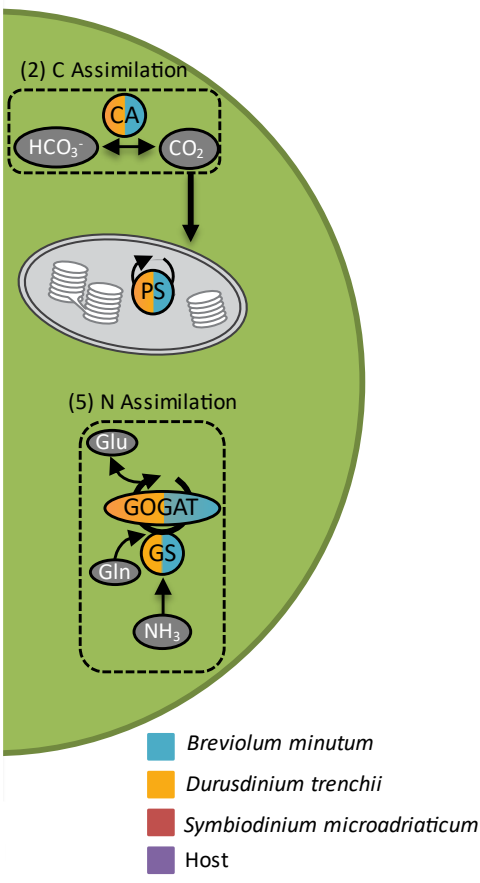

Figure 5.1. Conceptual diagram of the cellular processes under varying degrees of symbiont compatibility. Proteins from the host are shown in squares, and proteins from Symbiodiniaceae are shown in circles or ovals. Coloured proteins were significantly more abundant in the colour-coded treatments. Grey $=$ Molecules; Purple $=$ Host; Aqua $=$ Breviolum minutum; Gold = Durusdinium trenchii $;$ Red $=$ Symbiodinium microadriaticum. In a mutualistic association, (1) host vacuolar proton pumps (VHA), being part of the host carbon concentration mechanism $(\mathrm{CCM})$, transfer $\mathrm{H}^{+}$ions which acidify the symbiosome matrix ( $\mathrm{ph} \sim 4) ; \mathrm{V}=\mathrm{VHA}$. This facilitates conversion of bicarbonate ions $\left(\mathrm{HCO}_{3}{ }^{-}\right)$to carbon dioxide $\left(\mathrm{CO}_{2}\right)$ by carbonic anhydrase (CA), which can be further used by symbionts for photosynthesis. However, when ex hospite, $\mathrm{CA}$ in Symbiodiniaceae is upregulated to compensate for lack of readily available $\mathrm{CO}_{2}$ from the host (2). Photosynthates, such as glucose and glycerol, produced in photosynthesis are translocated to the host via host GLUT and AQP transporters (3); G = GLUT, AQ = AQP. Increase of host sugar transporters might be due to lower amounts of photosynthates translocated by the non-native symbionts. Nitrogen is provided to symbionts by host ammonium transporters (AM) (4), with the host expressing the lowest amount of AM when in hospite with the non-native $D$. trenchii. Ammonium is assimilated by the symbionts via the GS/GOGAT pathway (5), nevertheless, this pathway was upregulated only in the free-living state, mainly because the host controls the flow of nitrogen to its symbionts when in hospite. Phosphatidylinositol signalling system (PtdIns) used only by native symbionts for communication and cell recognition (6), while parasite-like host immunosuppression processes were only seen with non-native Durusdinium trenchii via histone $\mathrm{H} 4$ operating in an epigenetic manner (7). Economizing nutrient usage, such as for iron, by native Breviolum minutum under nutrient-limited conditions (8).

5.2.2 Does the degree of cellular integration between the symbiotic partners reflect the position of the symbiosis on the mutualism - parasitism continuum?

Increased sea surface temperatures are threating corals worldwide (Kleypas et al., 2021; Veron et al., 2009). However, the survival of corals may be achievable as posited in the Adaptive Bleaching Hypothesis (ABH) (Berkelmans and Van Oppen, 2006; Buddemeier et al., 2004; 
Buddemeier and Fautin, 1993). Nevertheless, the genetic diversity of Symbiodiniaceae, which strongly affects their functional diversity, raises questions about whether more thermally tolerant Symbiodiniaceae will be fully compatible at a cellular level with the majority of host species. Indeed, a high level of integration between host and symbiont is required for establishment of a novel symbiosis. Findings from this thesis and elsewhere (see below), however, suggest that the compatibility of a host and different species of symbionts is variable, with different host-symbiont combinations ranging from a mutualistic to a fully dysfunctional, perhaps parasitic state.

Studies in the past investigating the functional aspects of Symbiodiniaceae have reported that members of some genera, such as Symbiodinium and Durusdinium, have a more opportunistic, even parasitic nature, as these symbionts can reduce the fitness of the host even under normal conditions (Baker et al., 2018; Lesser et al., 2013; McIlroy et al., 2020; Stat et al., 2008). Conversely, these symbionts are among the most tolerant to environmental stress (Ghavam Mostafavi et al., 2007; Loram et al., 2007; Silverstein et al., 2017; Yuyama et al., 2016). Symbiodinium, being the most ancient genus, is very well adapted to high light conditions, however it can have free-living or opportunistic life-styles, likely due to lower physiological integration with the host in comparison to other recently evolved genera (Banaszak et al., 2000; LaJeunesse et al., 2018). In recent studies investigating the metabolome, colonization and establishment success of Symbiodiniaceae when inoculated into the model cnidarian Aiptasia, both Symbiodinium and Durusdinium spp. have been found to be relatively sub-optimal in this non-native partner (Gabay et al., 2018; Matthews et al., 2017). Similar findings exist for a more natural state, with background populations of Symbiodinium sp. in corals having lower rates of carbon fixation and translocation in comparison to Cladocopium spp. (Stat et al., 2008). Furthermore, Durusdinium sp., when living as a native symbiont of the corals Acropora and Isopora spp., is less efficient at nitrogen assimilation and photosynthate translocation than other native partners from the genus Cladocopium (Baker et al., 2013; Pernice et al., 2015).

Opportunistic and potentially parasitic strategies are also suggested by events during host colonisation. Symbiont cell density and proliferation are actively regulated by the host in a functional association (Davy et al., 2012; Detournay and Weis, 2011; Gorman et al., 2020; 
Muscatine and Pool, 1979; Tivey et al., 2020). Nevertheless, here D. trenchii was found to have variety of immunosuppressive mechanisms for evading the host cell immune response, all of which were similar to those reported for various microbial and viral pathogens (e.g., apicomplexans and polydnaviruses), and were exclusive to this species of Symbiodiniaceae (Chapter 3). In particular, incorporation of an epigenetic mode of immunosuppression by histone H4 was evident, which can suppress host cell development (Kumar et al., 2017) (Fig. 5.1). Sproles et al. (2019) similarly provided proteomic evidence for the interruption of the host cellular immune response, as well as protein and DNA synthesis, when Aiptasia was colonised by $D$. trenchii. Such suppression of the host's immune pathways might aid the unregulated proliferation of opportunistic symbionts, with an associated metabolic cost to the host (Matthews et al., 2017).

As discussed above (Section 5.2.1), and in Chapters $3 \&$ 4, the results in this thesis support the suggestion that certain species of Symbiodinium and Durusdinium may be less beneficial, opportunistic, and even parasitic, though caution should be exercised when extrapolating species-specific observations to the whole genus as we currently lack sufficient information to make such generalisations (Suggett et al., 2017). The ecological implications of these cellularlevel findings are discussed in the next section.

5.2.3 What are the ecological implications for the survival of coral reefs in the face of climate change, and the development of strategies for increasing reef resilience?

Stress tolerant Symbiodiniaceae, though potentially advantageous, can sometimes also be opportunistic and harmful to their cnidarian hosts, even under normal conditions (Gabay et al., 2018; Little et al., 2004; Pernice et al., 2015; Sproles et al., 2020; Starzak et al., 2014) (Chapters 3 \& 4). Nevertheless, when in hospite with their 'correct' hosts, these symbionts can have high nutritional value and confer thermal tolerance with respect to global warming (Baker et al., 2013; Chen et al., 2020; Hoadley et al., 2019; Silverstein et al., 2017). This thesis supports the view that only those corals that already harbour stress-tolerant Symbiodiniaceae, whether as the dominant or background member of their symbiont community, will ultimately reap the benefits. Nevertheless, it is predicted that Symbiodinium and Durusdinium species will become more globally dominant as a result of symbiont 'shuffling', with a concomitant decline 
in the diversity of both host and symbiont species (Brown et al., 1999; Gintert et al., 2018; Levitan et al., 2014; Pettay et al., 2015; Sampayo et al., 2016). For instance, Indo-Pacific populations of $D$. trenchii have been reported to spread to coral colonies of the Greater Caribbean as a result of bioinvasion and climate warming (Pettay et al., 2015). However, while corals harbouring these symbionts can tolerate temperatures $1-2{ }^{\circ} \mathrm{C}$ higher than other hostsymbiont associations, their calcification rate is significantly reduced, suggesting maladaptation (Pettay et al., 2015). Crucially, the rate at which our oceans are warming will likely preclude the evolution of novel host-symbiont partnerships, limiting the time needed for the level of cellular integration required for a novel, fully functional, ecologically successful symbiosis to arise (Hoegh-Guldberg et al., 2007; Kleypas et al., 2021). However, we currently do not know how much time is actually needed for such events beyond the short-term experiments described here and in previous work, necessitating future research.

Applying biomarkers is one approach to aid with the conservation and restoration of coral reefs (Parkinson et al., 2019). Proteins were one of the first biomarkers that were established in coral heat-stress studies (Cziesielski et al., 2019); however, new high-throughput technologies used in cnidarian-dinoflagellate research, such as those used in this thesis, provide more reliable, applicable protein-based markers. These biomarkers can be combined with gene-editing technologies such as CRISPR/Cas9 to aid the generation of functional, novel host-symbiont partnerships, potentially through the manipulation of inter-partner communication pathways, ultimately engineering new thermally-resistant symbioses (Rosset et al., 2021).

\subsection{Future work}

Discovery of host proteins that are important in different symbiotic states and under thermal stress has been achieved previously by LC-MS/MS proteomic analysis (Oakley et al., 2017, 2016; Peng et al., 2010; Sproles et al., 2019). A new method for analysing the proteome of the dinoflagellate symbiont was developed here (Chapters 2 \& 3). However, new LC-MS/MS methods for the investigation of specific proteins of interest from either the host or symbiont have yet to be established for this symbiosis, such as the application of targeted proteomics or immunoprecipitation mass spectrometry (Marx, 2013; Mohammed et al., 2016). These approaches could be crucial for understanding specific cellular pathways. Moreover, 100 
developing methods such as phosphoproteomics or proteogenomics (Hogrebe et al., 2018; Nesvizhskii, 2014), which would enable the characterisation of novel protein sequences and post-translational modifications, and help to elucidate mechanisms of pathway activation, would be invaluable. Likewise, immunolabelling would be a very useful tool for confirming whether or not some proteins are active extracellularly. A method for the quantification of specific cellular proteins by immunocytochemical imagery was applied to the cnidariandinoflagellate symbiosis for the first time in this thesis (Chapter 4), though further improvements of this method are needed to be able to quantify and localize proteins of interest in real-time (i.e. live) via confocal fluorescence microscopy (Kang et al., 2021; Rudkouskaya et al., 2018). Furthermore, there are many more transporters of interest than the handful localised here; an expanded study of these would help to elucidate both the nutritional and cell signalling events that occur at the host-symbiont interface. Lastly, comparative work with more symbiont types and host species (including corals), under different thermal regimes and timescales, is needed to test the generality of the observations presented here and their ecological relevance.

\subsection{Conclusions}

In conclusion, this thesis provides further evidence that incompatibility and reduced cellular integration between a host and non-native symbionts is likely to hinder nutritional exchange and induce cellular stress, ultimately limiting the potential for cnidarians to adapt to climate change by switching their dinoflagellate symbionts. My findings build on the work of Oakley et al. (2016), Baumgarten et al. (2015) and Sproles et al. (2019), who identified differential host immune and nutritional responses in Aiptasia in the presence of native and non-native species of symbionts, by providing evidence from the symbiont's perspective. Moreover, by applying previously published bioinformatics data (Sproles et al. 2018), I was able to visualise the influence of symbiont identity on the localisation and expression of key nutrient transporters in the symbiosis. We still have much to learn, however understanding the cellular and molecular mechanisms driving symbiosis specificity is hugely important. Indeed, it is essential if we are to truly understand the function and adaptive capacity of the cnidariandinoflagellate symbiosis and acquire the foundational knowledge necessary to develop tools that could help to save the world's coral reefs from climate change. 


\section{References}

Al-Moghrabi, S., Goiran, C., Allemand, D., Speziale, N., Jaubert, J., 1996. Inorganic carbon uptake for photosynthesis by the symbiotic coral- dinoflagellate association: II. Mechanisms for bicarbonate uptake. J. Exp. Mar. Bio. Ecol. 199, 227248. https://doi.org/10.1016/0022-0981(95)00202-2

Alberts, B., 2017. Molecular biology of the cell, Sixth. ed. Garland Science, Taylor \& Francis Group, New York, U.S.

Allemand, D., Furla, P., Bénazet-Tambutté, S., 1998. Mechanisms of carbon acquisition for endosymbiont photosynthesis in Anthozoa. Can. J. Bot. 76, 925-941.

Almaguer, C., Cheng, W., Nolder, C., Patton-Vogt, J., 2004. Glycerophosphoinositol, a novel phosphate source whose transport is regulated by multiple factors in Saccharomyces cerevisiae. J. Biol. Chem. 279, 31937-31942. https://doi.org/10.1074/jbc.M403648200

Andrade, S.L.A., Dickmanns, A., Ficner, R., Einsle, O., 2005. Crystal structure of the archaeal ammonium transporter Amt-1 from Archaeoglobus fulgidus. Proc. Natl. Acad. Sci. U. S. A. 102, 14994-14999. https://doi.org/10.1073/pnas.0506254102

Aranda, M., Li, Y., Liew, Y.J., Baumgarten, S., Simakov, O., Wilson, M.C., Piel, J., Ashoor, H., Bougouffa, S., Bajic, V.B., Ryu, T., Ravasi, T., Bayer, T., Micklem, G., Kim, H., Bhak, J., LaJeunesse, T.C., Voolstra, C.R., 2016. Genomes of coral dinoflagellate symbionts highlight evolutionary adaptations conducive to a symbiotic lifestyle. Sci. Rep. 6 , 39734. https://doi.org/10.1038/srep39734

Arena, E.T., Rueden, C.T., Hiner, M.C., Wang, S., Yuan, M., Eliceiri, K.W., 2017. Quantitating the cell: turning images into numbers with ImageJ. Wiley Interdiscip. Rev. Dev. Biol. 6, e260. https://doi.org/10.1002/wdev.260

Arif, C., Daniels, C., Bayer, T., Banguera-Hinestroza, E., Barbrook, A., Howe, C.J., Lajeunesse, T.C., Voolstra, C.R., 2014. Assessing Symbiodinium diversity in scleractinian corals via next-generation sequencing-based genotyping of the ITS2 rDNA region. Mol. Ecol. 23, 4418-4433. https://doi.org/10.1111/mec.12869

Arosio, P., Elia, L., Poli, M., 2017. Ferritin, cellular iron storage and regulation. IUBMB Life 69, 414-422. https://doi.org/10.1002/iub.1621

Augustin, R., 2010. The protein family of glucose transport facilitators: It's not only about glucose after all. IUBMB Life 62, 315-333. https://doi.org/10.1002/iub.315

Baird, A.H., Guest, J.R., Willis, B.L., 2009. Systematic and biogeographical patterns in the reproductive biology of scleractinian corals. Annu. Rev. Ecol. Evol. Syst. 40, 551-571. https://doi.org/10.1146/annurev.ecolsys.110308.120220

Baker, A.C., 2011. Zooxanthellae, in: Hopley, D. (Ed.), Encyclopedia of Earth Sciences Series. Springer Netherlands, Dordrecht, pp. 1189-1192. https://doi.org/10.1007/978-90-481-2639-2_280

Baker, A.C., 2003. Flexibility and Specificity in Coral-Algal Symbiosis: Diversity, Ecology, and Biogeography of Symbiodinium. Annu. Rev. Ecol. Evol. Syst. 34, 661-689. https://doi.org/10.1146/annurev.ecolsys.34.011802.132417

Baker, A.C., 2001. Ecosystems: Reef corals bleach to survive change. Nature 411, 765-766.

Baker, A.C., Glynn, P.W., Riegl, B., 2008. Climate change and coral reef bleaching: An ecological assessment of long-term impacts, recovery trends and future outlook. Estuar. Coast. Shelf Sci. 80, 435-471.

Baker, A.C., Starger, C.J., McClanahan, T.R., Glynn, P.W., 2004. Corals' adaptive response to climate change: Shifting to new algal symbionts may safeguard devastated reefs from extinction. Nature 430, 741. https://doi.org/10.1038/430741a

Baker, D.M., Andras, J.P., Jordán-Garza, A.G., Fogel, M.L., Guillermo Jordán-Garza, A., Fogel, M.L., 2013. Nitrate competition in a coral symbiosis varies with temperature among Symbiodinium clades. ISME J. 7, 1248-1251. https://doi.org/10.1038/ismej.2013.12

Baker, D.M., Freeman, C.J., Wong, J.C.Y., Fogel, M.L., Knowlton, N., 2018. Climate change promotes parasitism in a coral symbiosis. ISME J. 12, 921-930.

Banaszak, A.T., Iglestas-Prieto, R., Trench, R.K., 1993. Scrippsiella Velellae Sp. Nov. (Peridiniales) and Gloeokinium Viscum Sp. Nov. (Phytodiniales), Dinoflagellate Symbionts of Two Hydrozoans (Cnidiaria). J. Phycol. 29, 517-528. https://doi.org/10.1111/j.1529-8817.1993.tb00153.x

Banaszak, A.T., LaJeunesse, T.C., Trench, R.K., 2000. The synthesis of mycosporine-like amino acids (MAAS) by cultured, symbiotic dinoflagellates. J. Exp. Mar. Bio. Ecol. 249, 219-233. https://doi.org/10.1016/S0022-0981(00)00192-1 
Barott, K.L., Perez, S.O., Linsmayer, L.B., Tresguerres, M., 2015a. Differential localization of ion transporters suggests distinct cellular mechanisms for calcification and photosynthesis between two coral species. Am. J. Physiol. - Regul. Integr. Comp. Physiol. 309, R235-R246. https://doi.org/10.1152/ajpregu.00052.2015

Barott, K.L., Venn, A.A., Perez, S.O., Tambutteeé, S., Tresguerres, M., Somero, G.N., 2015b. Coral host cells acidify symbiotic algal microenvironment to promote photosynthesis. Proc. Natl. Acad. Sci. U. S. A. 112, 607-612. https://doi.org/10.1073/pnas.1413483112

Baumgarten, S., Simakov, O., Esherick, L.Y., Liew, Y.J., Lehnert, E.M., Michell, C.T., Li, Y., Hambleton, E.A., Guse, A., Oates, M.E., Gough, J., Weis, V.M., Aranda, M., Pringle, J.R., Voolstra, C.R., Knowlton, N., 2015. The genome of Aiptasia, a sea anemone model for coral symbiosis. Proc. Natl. Acad. Sci. U. S. A. 112, 11893-11898. https://doi.org/10.1073/pnas.1513318112

Bay, L.K., Cumbo, V.R., Abrego, D., Kool, J.T., Ainsworth, T.D., Willis, B.L., 2011. Infection dynamics vary between Symbiodinium types and cell surface treatments during establishment of endosymbiosis with coral larvae. Diversity 3 , 356-374.

Bay, R.A., Palumbi, S.R., 2015. Rapid acclimation ability mediated by transcriptome changes in reef-building corals. Genome Biol. Evol. 7, 1602-1612. https://doi.org/10.1093/gbe/evv085

Becker, F., Rhiel, E., 2006. Immuno-electron microscopic quantification of the fucoxanthin chlorophyll a/c binding polypeptides Fcp2, Fcp4, and Fcp6 of Cyclotella cryptica grown under low- and high-light intensities. Int. Microbiol. 9, 29-36. https://doi.org/10.1080/03057070500493753

Bednarz, V.N., Grover, R., Ferrier-Pagès, C., 2020. Elevated ammonium delays the impairment of the coral-dinoflagellate symbiosis during labile carbon pollution. Aquat. Toxicol. 218, 105360. https://doi.org/10.1016/j.aquatox.2019.105360

Beedessee, G., Hisata, K., Roy, M.C., Satoh, N., Shoguchi, E., 2015. Multifunctional polyketide synthase genes identified by genomic survey of the symbiotic dinoflagellate, Symbiodinium minutum. BMC Genomics 16, 941. https://doi.org/10.1186/s12864-015-2195-8

Bellantuono, A.J., Dougan, K.E., Granados-Cifuentes, C., Rodriguez-Lanetty, M., 2019. Free-living and symbiotic lifestyles of a thermotolerant coral endosymbiont display profoundly distinct transcriptomes under both stable and heat stress conditions. Mol. Ecol. 28, 5265-5281. https://doi.org/10.1111/mec.15300

Bellantuono, A.J., Hoegh-Guldberg, O., Rodriguez-Lanetty, M., 2012. Resistance to thermal stress in corals without changes in symbiont composition. Proc. R. Soc. B Biol. Sci. 279, 1100-1107. https://doi.org/10.1098/rspb.2011.1780

Berkelmans, R., Van Oppen, M.J.H., 2006. The role of zooxanthellae in the thermal tolerance of corals: A "nugget of hope" for coral reefs in an era of climate change. Proc. R. Soc. B Biol. Sci. 273, 2305-2312. https://doi.org/10.1098/rspb.2006.3567

Bernasconi, R., Stat, M., Koenders, A., Huggett, M.J., 2019. Global Networks of Symbiodinium-Bacteria Within the Coral Holobiont. Microb. Ecol. 77, 794-807. https://doi.org/10.1007/s00248-018-1255-4

Bertucci, A., Tambutté, É., Tambutté, S., Allemand, D., Zoccola, D., 2010. Symbiosis-dependent gene expression in coraldinoflagellate association: Cloning and characterization of a P-type H+-ATPase gene. Proc. R. Soc. B Biol. Sci. 277 , 87-95. https://doi.org/10.1098/rspb.2009.1266

Bhagooli, R., 2013. Inhibition of Calvin-Benson cycle suppresses the repair of photosystem II in Symbiodinium: Implications for coral bleaching. Hydrobiologia 714, 183-190. https://doi.org/10.1007/s10750-013-1535-4

Biquand, E., Okubo, N., Aihara, Y., Rolland, V., Hayward, D.C., Hatta, M., Minagawa, J., Maruyama, T., Takahashi, S., 2017. Acceptable symbiont cell size differs among cnidarian species and may limit symbiont diversity. ISME J. https://doi.org/10.1038/ismej.2017.17

Bishop, A.C., Sun, T., Johnson, M.E., Bruno, V.M., Patton-Vogt, J., 2011. Robust utilization of phospholipase-generated metabolites, glycerophosphodiesters, by Candida albicans: Role of the CaGit1 permease. Eukaryot. Cell 10, 1618 1627. https://doi.org/10.1128/EC.05160-11

Bjorkman, K., Karl, D.M., 1994. Bioavilability of inorganic and organic phosphorus compounds to natural assemblages of microorganisms in Hawaiian coastal waters. Mar. Ecol. Prog. Ser. 111, 265-274. https://doi.org/10.3354/meps111265

Blank, R.J., Trench, R.K., 1986. Nomenclature of Endosymbiotic Dinoflagellates. Taxon 35, 286-294. https://doi.org/10.2307/1221270

Boldt, L., Yellowlees, D., Leggat, W., 2012. Hyperdiversity of Genes Encoding Integral Light-Harvesting Proteins in the Dinoflagellate Symbiodinium sp. PLoS One 7, 1-13. https://doi.org/10.1371/journal.pone.0047456

Bolte, S., Cordelières, F.P., 2006. A guided tour into subcellular colocalization analysis in light microscopy. J. Microsc. 224, 
Borell, E.M., Bischof, K., 2008. Feeding sustains photosynthetic quantum yield of a scleractinian coral during thermal stress. Oecologia 157, 593-601. https://doi.org/10.1007/s00442-008-1102-2

Boulotte, N.M., Dalton, S.J., Carroll, A.G., Harrison, P.L., Putnam, H.M., Peplow, L.M., Van Oppen, M.J.H., 2016. Exploring the Symbiodinium rare biosphere provides evidence for symbiont switching in reef-building corals. ISME J. 10, 2693-2701. https://doi.org/10.1038/ismej.2016.54

Brandt, K., 1882. Über die morphologische und physiologische Bedeutung des Chlorophylls bei Thieren. Arch. Anat. Physiol. 4, 125-151.

Brown, B.E., Dunne, R.P., Ambarsari, I., Le Tissier, M.D.A., Satapoomin, U., 1999. Seasonal fluctuations in environmental factors and variations in symbiotic algae and chlorophyll pigments in four Indo-Pacific coral species. Mar. Ecol. Prog. Ser. 191, 53-69.

Bucher, M., Wolfowicz, I., Voss, P.A., Hambleton, E.A., Guse, A., 2016. Development and Symbiosis Establishment in the Cnidarian Endosymbiosis Model Aiptasia sp. Sci. Rep. 6, 19867. https://doi.org/10.1038/srep19867

Buchfink, B., Xie, C., Huson, D.H., 2014. Fast and sensitive protein alignment using DIAMOND. Nat. Methods 12, 59-60. https://doi.org/10.1038/nmeth.3176

Buddemeier, R.W., Baker, A.C., Fautin, D.G., Jacobs, J.R., 2004. The adaptive hypothesis of bleaching, in: Coral Health and Disease. Springer, pp. 427-444.

Buddemeier, R.W., Fautin, D.G., 1993. Coral Bleaching as an Adaptive Mechanism. Bioscience 43, 320-326. https://doi.org/10.2307/1312064

Burriesci, M.S., Raab, T.K., Pringle, J.R., 2012. Evidence that glucose is the major transferred metabolite in dinoflagellatecnidarian symbiosis. J. Exp. Biol. 215, 3467-3477. https://doi.org/10.1242/jeb.070946

Burry, R.W., 2009. Richard w. burry, SpringerLink : Bücher. Springer New York.

Byler, K.A., Carmi-Veal, M., Fine, M., Goulet, T.L., 2013. Multiple Symbiont Acquisition Strategies as an Adaptive Mechanism in the Coral Stylophora pistillata. PLoS One 8, e59596. https://doi.org/10.1371/journal.pone.0059596

Cabral, M., Anjard, C., Malhotra, V., Loomis, W.F., Kuspa, A., 2010. Unconventional secretion of AcbA in Dictyostelium discoideum through a vesicular intermediate. Eukaryot. Cell 9, 1009-1017. https://doi.org/10.1128/EC.00337-09

Camisão, A.F., Pedroso, C.C., 2013. Symbiosis: Evolution, Biology, and Ecological Effects, Animal Science, Issues and Professions. Nova Science.

Campbell, E.M., Ball, A., Hoppler, S., Bowman, A.S., 2008. Invertebrate aquaporins: A review. J. Comp. Physiol. B Biochem. Syst. Environ. Physiol. 178, 935-955. https://doi.org/10.1007/s00360-008-0288-2

Capasso, L., Ganot, P., Planas-Bielsa, V., Tambutté, S., Zoccola, D., 2021. Intracellular pH regulation: characterization and functional investigation of $\mathrm{H}+$ transporters in Stylophora pistillata. BMC Mol. Cell Biol. 22, 18. https://doi.org/10.1186/s12860-021-00353-x

Carbrey, J.M., Gorelick-Feldman, D.A., Kozono, D., Praetorius, J., Nielsen, S., Agre, P., 2003. Aquaglyceroporin AqP9: Solute permeation and metabolic control of expression in liver. Proc. Natl. Acad. Sci. U. S. A. 100, 2945-2950. https://doi.org/10.1073/pnas.0437994100

Carlos, A.A., Baillie, B.K., Kawachi, M., Maruyama, T., 1999. Phylogenetic position of Symbiodinium (Dinophyceae) isolates from Tridacnids (Bivalvia), Cardiids (Bivalvia), a sponge (Porifera), a soft coral (Anthozoa), and a free-living strain. J. Phycol. 35, 1054-1062. https://doi.org/10.1046/j.1529-8817.1999.3551054.x

Cates, N., McLaughlin, J.J.A., 1976. Differences of ammonia metabolism in symbiotic and aposymbiotic condylactus and cassiopea spp. J. Exp. Mar. Bio. Ecol. 21, 1-5. https://doi.org/10.1016/0022-0981(76)90065-4

Chan, H., Babayan, V., Blyumin, E., Gandhi, C., Hak, K., Harake, D., Kumar, K., Lee, P., Li, T.T., Liu, H.Y., Lo, T.C.T., Meyer, C.J., Stanford, S., Zamora, K.S., Saier, M.H., 2010. The P-Type ATPase superfamily. J. Mol. Microbiol. Biotechnol. 19, 5-104. https://doi.org/10.1159/000319588

Chen, B., Yu, K., Qin, Z., Liang, J., Wang, G., Huang, X., Wu, Q., Jiang, L., 2020. Dispersal, genetic variation, and symbiont interaction network of heat-tolerant endosymbiont Durusdinium trenchii: Insights into the adaptive potential of coral to climate change. Sci. Total Environ. 723, 138026. https://doi.org/https://doi.org/10.1016/j.scitotenv.2020.138026

Chen, H.K., Song, S.N., Wang, L.H., Mayfield, A.B., Chen, Y.J., Chen, W.N.U., Chen, C.S., 2015. A compartmental 
comparison of major lipid species in a coral-symbiodinium endosymbiosis: Evidence that the coral host regulates lipogenesis of its cytosolic lipid bodies. PLoS One 10, e0132519-e0132519.

https://doi.org/10.1371/journal.pone.0132519

Chen, M.C., Cheng, Y.M., Hong, M.C., Fang, L.S., 2004. Molecular cloning of Rab5 (ApRab5) in Aiptasia pulchella and its retention in phagosomes harboring live zooxanthellae. Biochem. Biophys. Res. Commun. 324, 1024-1033. https://doi.org/10.1016/j.bbrc.2004.09.151

Chen, M.C., Cheng, Y.M., Sung, P.J., Kuo, C.E., Fang, L.S., 2003. Molecular identification of Rab7 (ApRab7) in Aiptasia pulchella and its exclusion from phagosomes harboring zooxanthellae. Biochem. Biophys. Res. Commun. 308, 586595. https://doi.org/10.1016/S0006-291X(03)01428-1

Chen, M.C., Hong, M.C., Huang, Y. Sen, Liu, M.C., Cheng, Y.M., Fang, L.S., 2005. ApRab11, a cnidarian homologue of the recycling regulatory protein Rab11, is involved in the establishment and maintenance of the AiptasiaSymbiodinium endosymbiosis. Biochem. Biophys. Res. Commun. 338, 1607-1616. https://doi.org/10.1016/j.bbrc.2005.10.133

Chen, P.B., Ding, S., Zanghì, G., Soulard, V., DiMaggio, P.A., Fuchter, M.J., Mecheri, S., Mazier, D., Scherf, A., Malmquist, N.A., 2016. Plasmodium falciparum PfSET7: Enzymatic characterization and cellular localization of a novel protein methyltransferase in sporozoite, liver and erythrocytic stage parasites. Sci. Rep. 6, 1-14. https://doi.org/10.1038/srep21802

Chen, W.N.U., Kang, H.J., Weis, V.M., Mayfield, A.B., Jiang, P.L., Fang, L.S., Chen, C.S., 2012. Diel rhythmicity of lipidbody formation in a coral-Symbiodinium endosymbiosis. Coral Reefs 31, 521-534. https://doi.org/10.1007/s00338011-0868-6

Childress, J.J., Felbeck, H., Somero, G.N., 1987. Symbiosis in the Deep Sea. Sci. Am. 256, 115-120. https://doi.org/10.1038/scientificamerican0587-114

Cleves, P.A., Krediet, C.J., Lehnert, E.M., Onishi, M., Pringle, J.R., 2020. Insights into coral bleaching under heat stress from analysis of gene expression in a sea anemone model system. Proc. Natl. Acad. Sci. U. S. A. 117, 28906-28917. https://doi.org/10.1073/pnas.2015737117

Coker, D.J., Wilson, S.K., Pratchett, M.S., 2014. Importance of live coral habitat for reef fishes. Rev. Fish Biol. Fish. 24, 89-126. https://doi.org/10.1007/s11160-013-9319-5

Colley, N.J., Trench, R.K., 1983. Selectivity in phagocytosis and persistence of symbiotic algae in the scyphistoma stage of the jellyfish Cassiopeia xamachana. Proc. R. Soc. Lond. B. Biol. Sci. 219, 61-82. https://doi.org/10.1098/rspb.1983.0059

Comeau, S., Tambutté, E., Carpenter, R.C., Edmunds, P.J., Evensen, N.R., Allemand, D., Ferrier-Pagès, C., Tambutté, S., Venn, A.A., 2017. Coral calcifying fluid $\mathrm{pH}$ is modulated by seawater carbonate chemistry not solely seawater $\mathrm{pH}$. Proc. R. Soc. B Biol. Sci. 284. https://doi.org/10.1098/rspb.2016.1669

Comparot, S., Lingiah, G., Martin, T., 2003. Function and specificity of 14-3-3 proteins in the regulation of carbohydrate and nitrogen metabolism. J. Exp. Bot. 54, 595-604. https://doi.org/10.1093/jxb/erg057

Cook, C.B., 1971. Transfer of 35S-Labeled Material from Food Ingested by Aiptasia sp. to Its Endosymbiotic Zooxanthellae, in: Lenhoff, H., Muscatine, L., Davis, L. V (Eds.), . University of Hawaii Press, pp. 218-224. https://doi.org/doi:10.1515/9780824885335-023

Cook, C.B., D’Elia, C.F., Muller-Parker, G., 1988. Host feeding and nutrient sufficiency for zooxanthellae in the sea anemone Aiptasia pallida. Mar. Biol. 98, 253-262. https://doi.org/10.1007/BF00391203

Cooper, J.E., 2007. Early interactions between legumes and rhizobia: Disclosing complexity in a molecular dialogue. J. Appl. Microbiol. 103, 1355-1365. https://doi.org/10.1111/j.1365-2672.2007.03366.x

Cox, J., Hein, M.Y., Luber, C.A., Paron, I., Nagaraj, N., Mann, M., 2014. Accurate proteome-wide label-free quantification by delayed normalization and maximal peptide ratio extraction, termed MaxLFQ. Mol. Cell. Proteomics 13, 25132526. https://doi.org/10.1074/mcp.M113.031591

Crossland, C.J., Barnes, D.J., 1977. Nitrate assimilation enzymes from two hard corals, Acropora acuminata and Goniastrea australensis. Comp. Biochem. Physiol. -- Part B Biochem. 57, 151-157. https://doi.org/10.1016/0305-0491(77)901651

Cui, G., Liew, Y.J., Li, Y., Kharbatia, N., Zahran, N.I., Emwas, A.H., Eguiluz, V.M., Aranda, M., 2018. Meta-analysis reveals host-dependent nitrogen recycling as a mechanism of symbiont control in Aiptasia. bioRxiv 15, e1008189. https://doi.org/10.1101/269183

Cunning, R., Gillette, P., Capo, T., Galvez, K., Baker, A.C., 2015a. Growth tradeoffs associated with thermotolerant 105 
symbionts in the coral Pocillopora damicornis are lost in warmer oceans. Coral Reefs 34, 155-160. https://doi.org/10.1007/s00338-014-1216-4

Cunning, R., Silverstein, R.N., Baker, A.C., 2015b. Investigating the causes and consequences of symbiont shuffling in a multi-partner reef coral symbiosis under environmental change. Proc. R. Soc. B Biol. Sci. 282, 20141725. https://doi.org/10.1098/rspb.2014.1725

Cziesielski, M.J., Schmidt-Roach, S., Aranda, M., 2019. The past, present, and future of coral heat stress studies. Ecol. Evol. 9, 10055-10066. https://doi.org/10.1002/ece3.5576

D’Angelo, C., Hume, B.C.C., Burt, J., Smith, E.G., Achterberg, E.P., Wiedenmann, J., 2015. Local adaptation constrains the distribution potential of heat-tolerant Symbiodinium from the Persian/Arabian Gulf. ISME J. 9, 2551-2560. https://doi.org/10.1038/ismej.2015.80

D’Elia, C.F., Domotor, S.L., Webb, K.L., 1983. Nutrient uptake kinetics of freshly isolated zooxanthellae. Mar. Biol. 75, 157-167. https://doi.org/10.1007/BF00405998

Dani, V., Ganot, P., Priouzeau, F., Furla, P., Sabourault, C., 2014. Are Niemann-Pick type C proteins key players in cnidarian-dinoflagellate endosymbioses? Mol. Ecol. 23, 4527-4540. https://doi.org/10.1111/mec.12876

Dani, V., Priouzeau, F., Mertz, M., Mondin, M., Pagnotta, S., Lacas-Gervais, S., Davy, S.K., Sabourault, C., 2017. Expression patterns of sterol transporters NPC1 and NPC2 in the cnidarian-dinoflagellate symbiosis. Cell. Microbiol. 19. https://doi.org/10.1111/cmi.12753

Daniels, C., Baumgarten, S., Yum, L., MIchell, C., Bayer, T., Arif, C., Roder, C., Weil, E., Voolstra, C., 2015. Metatranscriptome analysis of the reef-building coral Orbicella faveolata indicates holobiont response to coral disease. Front. Mar. Sci.

Dassa, E., Bouige, P., 2001. The ABC of ABCs: A phylogenetic and functional classification of ABC systems in living organisms. Res. Microbiol. 152, 211-229. https://doi.org/10.1016/S0923-2508(01)01194-9

Davies, P.S., 1984. The role of zooxanthellae in the nutritional energy requirements of Pocillopora eydouxi. Coral Reefs 2 , 181-186. https://doi.org/10.1007/bf00263571

Davis, R.H., 2004. The age of model organisms. Nat. Rev. Genet. 5, 69-76. https://doi.org/10.1038/nrg1250

Davy, S.K., Allemand, D., Weis, V.M., 2012. Cell biology of cnidarian-dinoflagellate symbiosis. Microbiol. Mol. Biol. Rev. 76, 229-261.

Davy, S.K., Lucas, I.A.N., Turner, J.R., 1997. Uptake and persistence of homologous and heterologous zooxanthellae in the temperate sea anemone Cereus pedunculatus (Pennant). Biol. Bull. 192, 208-216. https://doi.org/10.2307/1542715

Davy, S.K., Turner, J.R., 2003. Early development and acquisition of zooxanthellae in the temperate symbiotic sea anemone Anthopleura ballii (Cocks). Biol. Bull. 205, 66-72. https://doi.org/10.2307/1543446

Davy, S.K., Withers, K.J.T., Hinde, R., 2006. Effects of host nutritional status and seasonality on the nitrogen status of zooxanthellae in the temperate coral Plesiastrea versipora (Lamarck). J. Exp. Mar. Bio. Ecol. 335, 256-265. https://doi.org/10.1016/j.jembe.2006.03.019

De Medina-Redondo, M., Arnáiz-Pita, Y., Fontaine, T., Del Rey, F., Latgé, J.P., De Aldana, C.R.V., 2008. The $\beta-1,3-$ glucanosyltransferase gas $4 \mathrm{p}$ is essential for ascospore wall maturation and spore viability in Schizosaccharomyces pombe. Mol. Microbiol. 68, 1283-1299. https://doi.org/10.1111/j.1365-2958.2008.06233.x

DeBosch, B.J., Chen, Z., Saben, J.L., Finck, B.N., Moley, K.H., 2014. Glucose transporter 8 (GLUT8) mediates fructoseinduced de Novo lipogenesis and macrosteatosis. J. Biol. Chem. 289, 10989-10998. https://doi.org/10.1074/jbc.M113.527002

Detournay, O., Weis, V.M., 2011. Role of the sphingosine rheostat in the regulation of cnidarian-dinoflagellate symbioses. Biol. Bull. 221, 261-269.

Díaz-Almeyda, E.M., Prada, C., Ohdera, A.H., Moran, H., Civitello, D.J., Iglesias-Prieto, R., Carlo, T.A., Lajeunesse, T.C., Medina, M., 2017. Intraspecific and interspecific variation in thermotolerance and photoacclimation in Symbiodinium dinoflagellates. Proc. R. Soc. B Biol. Sci. 284. https://doi.org/10.1098/rspb.2017.1767

Dick, G.J., 2019. The microbiomes of deep-sea hydrothermal vents: distributed globally, shaped locally. Nat. Rev. Microbiol. 17, 271-283.

Dilworth, J., Caruso, C., Kahkejian, V.A., Baker, A.C., Drury, C., 2021. Host genotype and stable differences in algal symbiont communities explain patterns of thermal stress response of Montipora capitata following thermal preexposure and across multiple bleaching events. Coral Reefs 40, 151-163. https://doi.org/10.1007/s00338-020-02024-3 
Dobson, S., Bracchi, V., Chakrabarti, D., Barik, S., 2001. Characterization of a novel serine/threonine protein phosphatase (PfPPJ) from the malaria parasite, Plasmodium falciparum. Mol. Biochem. Parasitol. 115, 29-39. https://doi.org/10.1016/S0166-6851(01)00260-2

Downs, C.A., Fauth, J.E., Halas, J.C., Dustan, P., Bemiss, J., Woodley, C.M., 2002. Oxidative stress and seasonal coral bleaching. Free Radic. Biol. Med. 33, 533-543. https://doi.org/10.1016/S0891-5849(02)00907-3

Drecktrah, D., Knodler, L.A., Steele-Mortimer, O., 2004. Modulation and utilization of host cell phosphoinositides by Salmonella spp. Infect. Immun. 72, 4331-4335.

Dubinsky, Z., Stambler, N., Ben-Zion, M., McCloskey, L.R., Muscatine, L., Falkowski, P.G., 1990. The effect of external nutrient resources on the optical properties and photosynthetic efficiency of Stylophora pistillata. Proc. R. Soc. B Biol. Sci. 239, 231-246. https://doi.org/10.1098/rspb.1990.0015

Dudler, N., Miller, D.J., 1988. Characterization of two glutamate dehydrogenases from the symbiotic microalga Symbiodinium microadriaticum isolated from the coral Acropora formosa. Mar. Biol. 97, 427-430. https://doi.org/10.1007/BF00397773

Dunn, S.R., 2009. Immunorecognition and immunoreceptors in the Cnidaria. Invertebr. Surviv. J. 6, 7-14.

Dunn, S.R., Schnitzler, C.E., Weis, V.M., 2007. Apoptosis and autophagy as mechanisms of dinoflagellate symbiont release during cnidarian bleaching: Every which way you lose. Proc. R. Soc. B Biol. Sci. 274, 3079-3085. https://doi.org/10.1098/rspb.2007.0711

Dunn, S.R., Thomas, M.C., Nette, G.W., Dove, S.G., 2012. A Lipidomic Approach to Understanding Free Fatty Acid Lipogenesis Derived from Dissolved Inorganic Carbon within Cnidarian-Dinoflagellate Symbiosis. PLoS One 7, e46801. https://doi.org/10.1371/journal.pone.0046801

Dunn, S.R., Thomason, J.C., Le Tissier, M.D.A., Bythell, J.C., 2004. Heat stress induces different forms of cell death in sea anemones and their endosymbiotic algae depending on temperature and duration. Cell Death Differ. 11, 1213-1222. https://doi.org/10.1038/sj.cdd.4401484

Duran, J.M., Anjard, C., Stefan, C., Loomis, W.F., Malhotra, V., 2010. Unconventional secretion of Acb1 is mediated by autophagosomes. J. Cell Biol. 188, 527-536. https://doi.org/10.1083/jcb.200911154

Ezawa, T., Hayatsu, M., Saito, M., 2005. A New Hypothesis on the Strategy for Acquisition of Phosphorus in Arbuscular Mycorrhiza: Up-Regulation of Secreted Acid Phosphatase Gene in the Host Plant. Mol. Plant-Microbe Interact. 18, 1046-1053. https://doi.org/10.1094/MPMI-18-1046

Ezzat, L., Fine, M., Maguer, J.F., Grover, R., Ferrier-Pagès, C., 2017. Carbon and nitrogen acquisition in shallow and deep holobionts of the scleractinian coral S. pistillata. Front. Mar. Sci. https://doi.org/10.3389/fmars.2017.00102

Ezzat, L., Maguer, J.F., Grover, R., Ferrier-Pagès, C., 2016. Limited phosphorus availability is the Achilles heel of tropical reef corals in a warming ocean. Sci. Rep. 6, 31768. https://doi.org/10.1038/srep31768

Falkowski, P.G., Dubinsky, Z., Muscatine, L., McCloskey, L., 1993. Population Control in Symbiotic Corals. Bioscience 43, 606-611. https://doi.org/10.2307/1312147

Ferrier-Pagès, C., Gattuso, J.P., Dallot, S., Jaubert, J., 2000. Effect of nutrient enrichment on growth and photosynthesis of the zooxanthellate coral Stylophora pistillata. Coral Reefs 19, 103-113. https://doi.org/10.1007/s003380000078

Ferrier-Pagès, C., Godinot, C., D’Angelo, C., Wiedenmann, J., Grover, R., 2016. Phosphorus metabolism of reef organisms with algal symbionts. Ecol. Monogr. 86, 262-277. https://doi.org/10.1002/ecm.1217

Ferrier-Pagès, C., Sauzéat, L., Balter, V., 2018. Coral bleaching is linked to the capacity of the animal host to supply essential metals to the symbionts. Glob. Chang. Biol. 24, 3145-3157. https://doi.org/10.1111/gcb.14141

Finazzi, G., Petroutsos, D., Tomizioli, M., Flori, S., Sautron, E., Villanova, V., Rolland, N., Seigneurin-Berny, D., 2015. Ions channels/transporters and chloroplast regulation. Cell Calcium 58, 86-97. https://doi.org/10.1016/j.ceca.2014.10.002

Finney, J.C., Pettay, D.T., Sampayo, E.M., Warner, M.E., Oxenford, H.A., LaJeunesse, T.C., 2010. The relative significance of host-habitat, depth, and geography on the ecology, endemism, and speciation of coral endosymbionts in the genus Symbiodinium. Microb. Ecol. 60, 250-263.

Fisher, P.L., Malme, M.K., Dove, S., 2012. The effect of temperature stress on coral-Symbiodinium associations containing distinct symbiont types. Coral Reefs 31, 473-485. https://doi.org/10.1007/s00338-011-0853-0

Fitt, W.K., Cook, C.B., 2001. The effects of feeding or addition of dissolved inorganic nutrients in maintaining the symbiosis between dinoflagellates and a tropical marine cnidarian. Mar. Biol. 139, 507-517. 
https://doi.org/10.1007/s002270100598

Fitt, W.K., Trench, R.K., 1983. Fate of ingestion symbiont by dinoflagellate.pdf. Cell Sci 212, 195-212.

Flessner, L.B., Moley, K.H., 2009. Similar [DE]XXXL[LI] motifs differentially target GLUT8 and GLUT12 in Chinese hamster ovary cells. Traffic 10, 324-333. https://doi.org/10.1111/j.1600-0854.2008.00866.x

Fontecave, M., Atta, M., Mulliez, E., 2004. S-adenosylmethionine: Nothing goes to waste. Trends Biochem. Sci. https://doi.org/10.1016/j.tibs.2004.03.007

Foyer, C.H., Shigeoka, S., 2011. Understanding oxidative stress and antioxidant functions to enhance photosynthesis. Plant Physiol. 155, 93-100. https://doi.org/10.1104/pp.110.166181

Franklin, D.J., Hoegh-Guldberg, O., Jones, R.J., Berges, J.A., 2004. Cell death and degeneration in the symbiotic dinoflagellates of the coral Stylophora pistillata during bleaching. Mar. Ecol. Prog. Ser. 272, 117-130. https://doi.org/10.3354/meps272117

Franklin, E.C., Stat, M., Pochon, X., Putnam, H.M., Gates, R.D., 2012. GeoSymbio: a hybrid, cloud-based web application of global geospatial bioinformatics and ecoinformatics for Symbiodinium-host symbioses. Mol. Ecol. Resour. 12, 369-373. https://doi.org/https://doi.org/10.1111/j.1755-0998.2011.03081.x

Fransolet, D., Roberty, S., Plumier, J.C., 2012. Establishment of endosymbiosis: The case of cnidarians and Symbiodinium. J. Exp. Mar. Bio. Ecol. 420-421, 1-7. https://doi.org/10.1016/j.jembe.2012.03.015

Fratti, R.A., Backer, J.M., Gruenberg, J., Corvera, S., Deretic, V., 2001. Role of phosphatidylinositol 3-kinase and Rab5 effectors in phagosomal biogenesis and mycobacterial phagosome maturation arrest. J. Cell Biol. 154, 631-644. https://doi.org/10.1083/jcb.200106049

Freudenthal, H.D., 1962. Symbiodinium gen. nov. and Symbiodinium microadriaticum sp. nov., a Zooxanthella: Taxonomy, Life Cycle, and Morphology. J. Protozool. 9, 45-52. https://doi.org/10.1111/j.1550-7408.1962.tb02579.x

Frøkiær, J., Kwon, T.H., Nielsen, J., Møller, H.B., Fenton, R.A., Nielsen, S., 2009. Aquaporins in the kidney, Handbook of Experimental Pharmacology, Handbook of Experimental Pharmacology. Springer Berlin Heidelberg. https://doi.org/10.1007/978-3-540-79885-9_5

Fu, Y., Zhao, W., Peng, Y., 2007. Induced expression of oryzain $\alpha$ gene encoding a cysteine proteinase under stress conditions. J. Plant Res. 120, 465-469. https://doi.org/10.1007/s10265-007-0080-5

Furla, P., Allemand, D., Orsenigo, M.N., 2000a. Involvement of H+-ATPase and carbonic anhydrase in inorganic carbon uptake for endosymbiont photosynthesis. Am. J. Physiol. - Regul. Integr. Comp. Physiol. 278, R870-R881. https://doi.org/10.1152/ajpregu.2000.278.4.r870

Furla, P., Galgani, I., Durand, I., Allemand, D., 2000b. Sources and mechanisms of inorganic carbon transport for coral calcification and photosynthesis. J. Exp. Biol. 203, 3445-3457.

Gabay, Y., Parkinson, J.E., Wilkinson, S.P., Weis, V.M., Davy, S.K., 2019. Inter-partner specificity limits the acquisition of thermotolerant symbionts in a model cnidarian-dinoflagellate symbiosis. ISME J. 13, 2489-2499. https://doi.org/10.1038/s41396-019-0429-5

Gabay, Y., Weis, V.M., Davy, S.K., 2018. Symbiont Identity Influences Patterns of Symbiosis Establishment, Host Growth, and Asexual Reproduction in a Model Cnidarian-Dinoflagellate Symbiosis. Biol. Bull. 234, 1-10. https://doi.org/10.1086/696365

Gad, W., Kim, Y., 2009. N-terminal tail of a viral histone H4 encoded in Cotesia plutellae bracovirus is essential to suppress gene expression of host histone H4. Insect Mol. Biol. 18, 111-118. https://doi.org/10.1111/j.1365-2583.2009.00860.x

Gad, W., Kim, Y., 2008. A viral histone H4 encoded by Cotesia plutellae bracovirus inhibits haemocyte-spreading behaviour of the diamondback moth, Plutella xylostella. J. Gen. Virol. 89, 931-938.

Galat, A., 1993. Peptidylproline cis-trans-isomerases: immunophilins. Eur. J. Biochem. 216, 689-707.

Galliot, B., 2012. Hydra, a fruitful model system for 270 years. Int. J. Dev. Biol. 56, 411-423. https://doi.org/10.1387/ijdb.120086bg

Gambardella, C., Gallus, L., Amaroli, A., Terova, G., Masini, M.A., Ferrando, S., 2012. Fasting and re-feeding impact on leptin and aquaglyceroporin 9 in the liver of European sea bass (Dicentrarchus labrax). Aquaculture 354-355, 1-6. https://doi.org/10.1016/j.aquaculture.2012.04.043

Ghavam Mostafavi, P., Fatemi, S.M.R., Shahhosseiny, M.H., Hoegh-Guldberg, O., Loh, W.K.W., 2007. Predominance of clade D Symbiodinium in shallow-water reef-building corals off Kish and Larak Islands (Persian Gulf, Iran). Mar. 
Biol. 153, 25-34. https://doi.org/10.1007/s00227-007-0796-8

Gierz, S.L., Forêt, S., Leggat, W., 2017. Transcriptomic Analysis of Thermally Stressed Symbiodinium Reveals Differential Expression of Stress and Metabolism Genes. Front. Plant Sci.

Gintert, B.E., Manzello, D.P., Enochs, I.C., Kolodziej, G., Carlton, R., Gleason, A.C.R., Gracias, N., 2018. Marked annual coral bleaching resilience of an inshore patch reef in the Florida Keys: A nugget of hope, aberrance, or last man standing? Coral Reefs 37, 533-547. https://doi.org/10.1007/s00338-018-1678-x

Glider, W. V., Phipps, D.W., Pardy, R.L., 1980. Localization of Symbiotic Dinoflagellate Cells within Tentacle Tissue of Aiptasia pallida (Coelenterata, Anthozoa). Trans. Am. Microsc. Soc. 99, 426. https://doi.org/10.2307/3225653

Glynn, P.W., 1993. Coral reef bleaching: ecological perspectives. Coral Reefs 12, 1-17. https://doi.org/10.1007/BF00303779

Godinot, C., Ferrier-Pagès, C., Grover, R., 2009. Control of phosphate uptake by zooxanthellae and host cells in the scleractinian coral Stylophora pistillata. Limnol. Oceanogr. 54, 1627-1633. https://doi.org/10.4319/lo.2009.54.5.1627

Goiran, C., Allemand, D., Galgani, I., 1997. Transient Na+ stress in symbiotic dinoflagellates after isolation from coral-host cells and subsequent immersion in seawater. Mar. Biol. 129, 581-589. https://doi.org/10.1007/s002270050199

Gorman, L.M., Wilkinson, S.P., Kitchen, S.A., Oakley, C.A., Grossman, A.R., Weis, V.M., Davy, S.K., 2020. Phylogenetic analysis of cell-cycle regulatory proteins within the Symbiodiniaceae. Sci. Rep. 10, 1-13.

Graham, L.A., Flannery, A.R., Stevens, T.H., 2003. Structure and Assembly of the Yeast V-ATPase. J. Bioenerg. Biomembr. 35, 301-312. https://doi.org/10.1023/A:1025772730586

Grajales, A., Rodríguez, E., 2014. Morphological revision of the genus Aiptasia and the family Aiptasiidae (Cnidaria, Actiniaria, etridioidea). Zootaxa 3826, 55-100. https://doi.org/10.11646/zootaxa.3826.1.2

Grégoire, V., Schmacka, F., Coffroth, M.A., Karsten, U., 2017. Photophysiological and thermal tolerance of various genotypes of the coral endosymbiont Symbiodinium sp. (Dinophyceae). J. Appl. Phycol. 29, 1893-1905. https://doi.org/10.1007/s10811-017-1127-1

Groussman, R.D., Parker, M.S., Armbrust, E.V., 2015. Diversity and evolutionary history of iron metabolism genes in diatoms. PLoS One 10, e0129081. https://doi.org/10.1371/journal.pone.0129081

Grover, R., Maguer, J.-F.F., Allemand, D., Ferrier-Pagès, C., 2008. Uptake of dissolved free amino acids by the scleractinian coral Stylophora pistillata. J. Exp. Biol. 211, 860-865. https://doi.org/10.1242/jeb.012807

Grover, R., Maguer, J.F., Allemand, D., Ferrier-Pagès, C., 2003. Nitrate uptake in the scleractinian coral Stylophora pistillata. Limnol. Oceanogr. 48, 2266-2274. https://doi.org/10.4319/1o.2003.48.6.2266

Grover, R., Maguer, J.F., Reynaud-Vaganay, S., Ferrier-Pagès, C., 2002. Uptake of ammonium by the scleractinian coral Stylophora pistillata: Effect of feeding, light, and ammonium concentrations. Limnol. Oceanogr. 47, 782-790. https://doi.org/10.4319/1o.2002.47.3.0782

Guerrero, R., Margulis, L., Berlanga, M., 2013. Symbiogenesis: The holobiont as a unit of evolution. Int. Microbiol. 16, 133-143. https://doi.org/10.2436/20.1501.01.188

Guillard, R.R.L., 1975. Culture of Phytoplankton for Feeding Marine Invertebrates, in: Culture of Marine Invertebrate Animals. Springer, pp. 29-60. https://doi.org/10.1007/978-1-4615-8714-9 3

Hansen, G., Daugbjerg, N., 2009. Symbiodinium natans SP. Nov.: A “free-living” dinoflagellate from tenerife (northeastatlantic ocean). J. Phycol. 45, 251-263. https://doi.org/10.1111/j.1529-8817.2008.00621.x

Harland, A.D., Davies, P.S., 1995. Symbiont photosynthesis increases both respiration and photosynthesis in the symbiotic sea anemone Anemonia viridis. Mar. Biol. 123, 715-722. https://doi.org/10.1007/BF00349114

Harvey, K.L., Jarocki, V.M., Charles, I.G., Djordjevic, S.P., 2019. The diverse functional roles of elongation factor tu (Ef-tu) in microbial pathogenesis. Front. Microbiol. 10, 2351. https://doi.org/10.3389/fmicb.2019.02351

Hawkins, T.D., Bradley, B.J., Davy, S.K., 2013. Nitric oxide mediates coral bleaching through an apoptotic-like cell death pathway: Evidence from a model sea anemone-dinoflagellate symbiosis. FASEB J. 27, 4790-4798. https://doi.org/10.1096/fj.13-235051

Herbik, A., Bölling, C., Buckhout, T.J., 2002. The involvement of a multicopper oxidase in iron uptake by the green algae Chlamydomonas reinhardtii. Plant Physiol. 130, 2039-2048. https://doi.org/10.1104/pp.013060

Hibuse, T., Maeda, N., Nagasawa, A., Funahashi, T., 2006. Aquaporins and glycerol metabolism. Biochim. Biophys. Acta Biomembr. 1758, 1004-1011. https://doi.org/10.1016/j.bbamem.2006.01.008 
Higgins, C.F., 1992. ABC Transporters: From microorganisms to man. Annu. Rev. Cell Biol. 8, 67-113. https://doi.org/10.1146/annurev.cb.08.110192.000435

Hilbi, H., 2006. Modulation of phosphoinositide metabolism by pathogenic bacteria. Cell. Microbiol. 8, 1697-1706. https://doi.org/10.1111/j.1462-5822.2006.00793.x

Hillyer, K.E., 2016. Thermal Stress and Bleaching in the Cnidarian-Dinoflagellate Symbiosis: The Application of Metabolomics. https://doi.org/10.1017/CBO9781107415324.004

Hillyer, K.E., Dias, D.A., Lutz, A., Roessner, U., Davy, S.K., 2017a. Mapping carbon fate during bleaching in a model cnidarian symbiosis: the application of 13C metabolomics. New Phytol. 214, 1551-1562. https://doi.org/10.1111/nph.14515

Hillyer, K.E., Dias, D.A., Lutz, A., Wilkinson, S.P., Roessner, U., Davy, S.K., 2017b. Metabolite profiling of symbiont and host during thermal stress and bleaching in the coral Acropora aspera. Coral Reefs 36, 105-118. https://doi.org/10.1007/s00338-016-1508-y

Hillyer, K.E., Tumanov, S., Villas-Bôas, S., Davy, S.K., 2016. Metabolite profiling of symbiont and host during thermal stress and bleaching in a model cnidarian-dinoflagellate symbiosis. J. Exp. Biol. 219, 516-527. https://doi.org/10.1242/jeb.128660

Hoadley, K.D., Lewis, A.M., Wham, D.C., Pettay, D.T., Grasso, C., Smith, R., Kemp, D.W., LaJeunesse, T.C., Warner, M.E., 2019. Host-symbiont combinations dictate the photo-physiological response of reef-building corals to thermal stress. Sci. Rep. 9, 1-15.

Hoegh-Guldberg, O., 1999. Climate change, coral bleaching and the future of the world's coral reefs. Mar. Freshw. Res. 50, 839-866.

Hoegh-Guldberg, O., 1994. Population Dynamics of Symbiotic Zooxanthellae in the Coral Pocillopora damicornis Exposed to Elevated Ammonium [(NH4)2 SO4] Concentrations. Pacific Sci. 48.

Hoegh-Guldberg, O., Jones, R.J., Ward, S., Loh, W.K., Baker, A.C., 2002. Communicating arising ecology: Is coral bleaching really adaptive? Nature 415, 601-602. https://doi.org/10.1038/415601a

Hoegh-Guldberg, O., Mumby, P.J., Hooten, A.J., Steneck, R.S., Greenfield, P., Gomez, E., Harvell, C.D., Sale, P.F., Edwards, A.J., Caldeira, K., Knowlton, N., Eakin, C.M., Iglesias-Prieto, R., Muthiga, N., Bradbury, R.H., Dubi, A., Hatziolos, M.E., 2007. Coral reefs under rapid climate change and ocean acidification. Science 318, 1737-1742. https://doi.org/10.1126/science.1152509

Hoegh-Guldberg, O., Poloczanska, E.S., Skirving, W., Dove, S., 2017. Coral reef ecosystems under climate change and ocean acidification. Front. Mar. Sci. 4, 158. https://doi.org/10.3389/fmars.2017.00158

Hofmann, D.K., Kremer, B.P., 1981. Carbon metabolism and strobilation in Cassiopea andromedea (Cnidaria: Scyphozoa): Significance of endosymbiotic dinoflagellates. Mar. Biol. 65, 25-33. https://doi.org/10.1007/BF00397064

Hogrebe, A., Von Stechow, L., Bekker-Jensen, D.B., Weinert, B.T., Kelstrup, C.D., Olsen, J. V., 2018. Benchmarking common quantification strategies for large-scale phosphoproteomics. Nat. Commun. 9. https://doi.org/10.1038/s41467-018-03309-6

Hohman, T.C., McNeil, P.L., Muscatine, L., 1982. Phagosome-lysosome fusion inhibited by algal symbionts of hydra Viridis. J. Cell Biol. 94, 56-63. https://doi.org/10.1083/jcb.94.1.56

Hoogenboom, M.O., Campbell, D.A., Beraud, E., DeZeeuw, K., Ferrier-Pagès, C., 2012. Effects of light, food availability and temperature stress on the function of photosystem II and photosystem I of coral symbionts. PLoS One 7, e30167. https://doi.org/10.1371/journal.pone.0030167

Hoppert, M., Wrede, C., 2011. Immunolocalization, in: Reitner, J., Thiel, V. (Eds.), Encyclopedia of Earth Sciences Series. Springer Netherlands, Dordrecht, pp. 482-486. https://doi.org/10.1007/978-1-4020-9212-1_116

Howden, A.J.M., Preston, G.M., 2009. Nitrilase enzymes and their role in plant-microbe interactions. Microb. Biotechnol. 2, 441-451. https://doi.org/10.1111/j.1751-7915.2009.00111.x

Huang, N., Ledyard Stebbins, G., Rodriguez, R.L., 1992. Classification and evolution of $\alpha$-amylase genes in plants. Proc. Natl. Acad. Sci. U. S. A. 89, 7526-7530. https://doi.org/10.1073/pnas.89.16.7526

Hughes, T.P., Baird, A.H., Bellwood, D.R., Card, M., Connolly, S.R., Folke, C., Grosberg, R., Hoegh-Guldberg, O., Jackson, J.B.C., Kleypas, J., Lough, J.M., Marshall, P., Nyström, M., Palumbi, S.R., Pandolfi, J.M., Rosen, B., Roughgarden, J., 2003. Climate Change, Human Impacts, and the Resilience of Coral Reefs. Science (80-. ). 301, 929-933. https://doi.org/10.1126/science.1085046 
Hughes, T.P., Kerry, J.T., Álvarez-Noriega, M., Álvarez-Romero, J.G., Anderson, K.D., Baird, A.H., Babcock, R.C., Beger, M., Bellwood, D.R., Berkelmans, R., Bridge, T.C., Butler, I.R., Byrne, M., Cantin, N.E., Comeau, S., Connolly, S.R., Cumming, G.S., Dalton, S.J., Diaz-Pulido, G., Eakin, C.M., Figueira, W.F., Gilmour, J.P., Harrison, H.B., Heron, S.F., Hoey, A.S., Hobbs, J.P.A., Hoogenboom, M.O., Kennedy, E. V., Kuo, C.Y., Lough, J.M., Lowe, R.J., Liu, G., McCulloch, M.T., Malcolm, H.A., McWilliam, M.J., Pandolfi, J.M., Pears, R.J., Pratchett, M.S., Schoepf, V., Simpson, T., Skirving, W.J., Sommer, B., Torda, G., Wachenfeld, D.R., Willis, B.L., Wilson, S.K., 2017. Global warming and recurrent mass bleaching of corals. Nature 543, 373-377. https://doi.org/10.1038/nature21707

Hughes, T.P., Kerry, J.T., Simpson, T., 2018. Large-scale bleaching of corals on the Great Barrier Reef. Ecology 99, 501. https://doi.org/10.1002/ecy.2092

Hume, B., D’angelo, C., Burt, J., Baker, A.C., Riegl, B., Wiedenmann, J., 2013. Corals from the Persian/Arabian Gulf as models for thermotolerant reef-builders: prevalence of clade C3 Symbiodinium, host fluorescence and ex situ temperature tolerance. Mar. Pollut. Bull. 72, 313-322.

Hume, B.C.C., D’Angelo, C., Smith, E.G., Stevens, J.R., Burt, J., Wiedenmann, J., 2015. Symbiodinium thermophilum sp. nov., a thermotolerant symbiotic alga prevalent in corals of the world's hottest sea, the Persian/Arabian Gulf. Sci. Rep. 5, 8562. https://doi.org/10.1038/srep08562

Ibberson, M., Riederer, B.M., Uldry, M., Guhl, B., Roth, J., Thorens, B., 2002. Immunolocalization of GLUTX1 in the testis and to specific brain areas and vasopressin-containing neurons. Endocrinology 143, 276-284. https://doi.org/10.1210/endo.143.1.8587

Ibberson, M., Uldry, M., Thorens, B., 2000. GLUTX1, a novel mammalian glucose transporter expressed in the central nervous system and insulin-sensitive tissues. J. Biol. Chem. 275, 4607-4612. https://doi.org/10.1074/jbc.275.7.4607

Imbs, A.B., Yakovleva, I.M., Dautova, T.N., Bui, L.H., Jones, P., 2014. Diversity of fatty acid composition of symbiotic dinoflagellates in corals: Evidence for the transfer of host PUFAs to the symbionts. Phytochemistry 101, 76-82. https://doi.org/10.1016/j.phytochem.2014.02.012

Ip, Y.K., Hiong, K.C., Lim, L.J.Y., Choo, C.Y.L., Boo, M. V., Wong, W.P., Neo, M.L., Chew, S.F., 2018. Molecular characterization, light-dependent expression, and cellular localization of a host vacuolar-type H+-ATPase (VHA) subunit A in the giant clam, Tridacna squamosa, indicate the involvement of the host VHA in the uptake of inorganic carbon and its su. Gene 659, 137-148. https://doi.org/10.1016/j.gene.2018.03.054

Ishibashi, K., Kondo, S., Hara, S., Morishita, Y., 2011. The evolutionary aspects of aquaporin family. Am. J. Physiol. Regul. Integr. Comp. Physiol. 300, R566-R576. https://doi.org/10.1152/ajpregu.90464.2008

Jackson, A.E., Miller, D.J., Yellowlees, D., 1989. Phosphorus metabolism in the coral-zooxanthellae symbiosis: Characterization and possible roles of two acid phosphatases in the algal symbiont Symbiodinium sp. Proc. R. Soc. B Biol. Sci. 238, 193-202. https://doi.org/10.1098/rspb.1989.0076

Jackson, A.E., Yellowlees, D., 1990. Phosphate uptake by zooxanthellae isolated from corals. Proc. R. Soc. B Biol. Sci. 242, 201-204. https://doi.org/10.1098/rspb.1990.0125

Jeong, H.J., Yoo, Y. Du, Kang, N.S., Lim, A.S., Seong, K.A., Lee, S.Y., Lee, M.J., Lee, K.H., Kim, H.S., Shin, W., Nam, S.W., Yih, W., Lee, K., 2012. Heterotrophic feeding as a newly identified survival strategy of the dinoflagellate Symbiodinium. Proc. Natl. Acad. Sci. U. S. A. 109, 12604-12609. https://doi.org/10.1073/pnas.1204302109

Johannes, R.E., Coles, S.L., Kuenzel, N.T., 1970. The Role of Zooplankton in the Nutrition of Some Scleractinian Corals. Limnol. Oceanogr. 579-586.

Jones, A.M., Berkelmans, R., 2011. Tradeoffs to thermal acclimation: energetics and reproduction of a reef coral with heat tolerant Symbiodinium type-D. J. Mar. Biol. 2011.

Jonkman, J., Brown, C.M., Wright, G.D., Anderson, K.I., North, A.J., 2020. Tutorial: guidance for quantitative confocal microscopy. Nat. Protoc. 15, 1585-1611. https://doi.org/10.1038/s41596-020-0313-9

Kahng, S.E., Garcia-Sais, J.R., Spalding, H.L., Brokovich, E., Wagner, D., Weil, E., Hinderstein, L., Toonen, R.J., 2010. Community ecology of mesophotic coral reef ecosystems. Coral Reefs 29, 255-275. https://doi.org/10.1007/s00338010-0593-6

Kaiser, P., Schlichter, D., Fricke, H.W., 1993. Influence of light on algal symbionts of the deep water coral Leptoseris fragilis. Mar. Biol. 117, 45-52. https://doi.org/10.1007/BF00346424

Kamke, J., Sczyrba, A., Ivanova, N., Schwientek, P., Rinke, C., Mavromatis, K., Woyke, T., Hentschel, U., 2013. Single-cell genomics reveals complex carbohydrate degradation patterns in poribacterial symbionts of marine sponges. ISME J. 7, 2287-2300. https://doi.org/10.1038/ismej.2013.111

Kang, J.W., Nguyen, F.T., Lue, N., 2021. Temporal Imaging of Live Cells by High-Speed Confocal Raman Microscopy. 
Materials (Basel). https://doi.org/10.3390/ma14133732

Karako-Lampert, S., Katcoff, D.J., Achituv, Y., Dubinsky, Z., Stambler, N., 2005. Responses of Symbiodinium microadriaticum clade B to different environmental conditions. J. Exp. Mar. Bio. Ecol. 318, 11-20. https://doi.org/10.1016/j.jembe.2004.11.024

Kawaguti, S., 1944. On the physiology of reef corals. VII. Zooxanthellae of the reef corals Gymnodinium sp. , Dinoflagellata. Its culture in vitro. Palaeo Trop Biol Stat Stud 2, 675-679 (travail non consulté).

Kazandjian, A., Shepherd, V.A., Rodriguez-Lanetty, M., Nordemeier, W., Larkum, A.W.D., Quinnell, R.G., 2008. Isolation of symbiosomes and the symbiosome membrane complex from the zoanthid Zoanthus robustus. Phycologia 47, 294 306. https://doi.org/10.2216/PH07-23.1

Kelley, J.B., Paschal, B.M., 2019. Fluorescence-based quantification of nucleocytoplasmic transport. Methods 157, 106114. https://doi.org/10.1016/j.ymeth.2018.11.002

Kelly, J.G., Hawken, M.J., 2017. Quantification of neuronal density across cortical depth using automated 3D analysis of confocal image stacks. Brain Struct. Funct. 222, 3333-3353. https://doi.org/10.1007/s00429-017-1382-6

Kemp, D.W., Hernandez-Pech, X., Iglesias-Prieto, R., Fitt, W.K., Schmidt, G.W., 2014. Community dynamics and physiology of Symbiodinium spp. before, during, and after a coral bleaching event. Limnol. Oceanogr. 59, 788-797.

Kennedy, E. V., Foster, N.L., Mumby, P.J., Stevens, J.R., 2015. Widespread prevalence of cryptic Symbiodinium D in the key Caribbean reef builder, Orbicella annularis. Coral Reefs 34, 519-531. https://doi.org/10.1007/s00338-015-1264-4

Kilpatrick, D.C., 2002. Animal lectins: A historical introduction and overview. Biochim. Biophys. Acta - Gen. Subj. 1572, 187-197. https://doi.org/10.1016/S0304-4165(02)00308-2

Kim, J., Kim, Y., 2010. A viral histone H4 suppresses expression of a transferrin that plays a role in the immune response of the diamondback moth, Plutella xylostella. Insect Mol. Biol. 19, 567-574. https://doi.org/10.1111/j.13652583.2010.01014.x

Kirk, N.L., Weis, V.M., 2016. Animal-Symbiodinium Symbioses: Foundations of Coral Reef Ecosystems, in: Hurst, C.J. (Ed.), . Springer International Publishing, Cham, pp. 269-294. https://doi.org/10.1007/978-3-319-28068-4_10

Kitchen, S.A., Crowder, C.M., Poole, A.Z., Weis, V.M., Meyer, E., 2015. De novo assembly and characterization of four anthozoan (phylum Cnidaria) transcriptomes. G3 Genes, Genomes, Genet. 5, 2441-2452. https://doi.org/10.1534/g3.115.020164

Klee, C.B., Ren, H., Wang, X., 1998. Regulation of the calmodulin-stimulated protein phosphatase, calcineurin. J. Biol. Chem. 273, 13367-13370. https://doi.org/10.1074/jbc.273.22.13367

Kleypas, J., Allemand, D., Anthony, K., Baker, A.C., Beck, M.W., Hale, L.Z., Hilmi, N., Hoegh-Guldberg, O., Hughes, T., Kaufman, L., Kayanne, H., Magnan, A.K., Mcleod, E., Mumby, P., Palumbi, S., Richmond, R.H., Rinkevich, B., Steneck, R.S., Voolstra, C.R., Wachenfeld, D., Gattuso, J.P., 2021. Designing a blueprint for coral reef survival. Biol. Conserv. 257. https://doi.org/10.1016/j.biocon.2021.109107

Kloc, M., 2020. Symbiosis: Cellular, Molecular, Medical and Evolutionary Aspects.

Kopp, C., Pernice, M., Domart-Coulon, I., Djediat, C., Spangenberg, J.E., Alexander, D.T.L., Hignette, M., Meziane, T., Meibom, A., 2013. Highly dynamic cellular-level response of symbiotic coral to a sudden increase in environmental nitrogen. MBio 4, e00052-13. https://doi.org/10.1128/mBio.00052-13

Kroh, G.E., Pilon, M., 2020. Regulation of iron homeostasis and use in chloroplasts. Int. J. Mol. Sci. 21. https://doi.org/10.3390/ijms21093395

Krueger, T., 2017. Concerning the cohabitation of animals and algae - an English translation of K . Brandt's 1881 presentation B Ueber das Zusammenleben von Thieren und Algen ^ 167-174. https://doi.org/10.1007/s13199-016$0439-2$

Krueger, T., Becker, S., Pontasch, S., Dove, S., Hoegh-Guldberg, O., Leggat, W., Fisher, P.L., Davy, S.K., 2014. Antioxidant plasticity and thermal sensitivity in four types of Symbiodinium sp. J. Phycol. 50, 1035-1047. https://doi.org/10.1111/jpy.12232

Kruger, N.J., Von Schaewen, A., 2003. The oxidative pentose phosphate pathway: Structure and organisation. Curr. Opin. Plant Biol. 6, 236-246. https://doi.org/10.1016/S1369-5266(03)00039-6

Kumar, S., Jung, J.K., Kim, Y., 2017. Characterization of joining sites of a viral histone H4 on host insect chromosomes. PLoS One 12, 1-28. https://doi.org/10.1371/journal.pone.0177066 
Kuriyama, H., Shimomura, I., Kishida, K., Kondo, H., Furuyama, N., Nishizawa, H., Maeda, N., Matsuda, M., Nagaretani, H., Kihara, S., Nakamura, T., Tochino, Y., Funahashi, T., Matsuzawa, Y., 2002. Coordinated regulation of fat-specific and liver-specific glycerol channels, aquaporin adipose and aquaporin 9. Diabetes 51, 2915-2921. https://doi.org/10.2337/diabetes.51.10.2915

Labourel, A., Frandsen, K.E.H., Zhang, F., Brouilly, N., Grisel, S., Haon, M., Ciano, L., Ropartz, D., Fanuel, M., Martin, F., Navarro, D., Rosso, M.N., Tandrup, T., Bissaro, B., Johansen, K.S., Zerva, A., Walton, P.H., Henrissat, B., Leggio, L. Lo, Berrin, J.G., 2020. A fungal family of lytic polysaccharide monooxygenase-like copper proteins. Nat. Chem. Biol. 16, 345-350. https://doi.org/10.1038/s41589-019-0438-8

LaJeunesse, T.C., 2005. "Species" Radiations of Symbiotic Dinoflagellates in the Atlantic and Indo-Pacific Since the Miocene-Pliocene Transition. Mol. Biol. Evol. 22, 570-581. https://doi.org/10.1093/molbev/msi042

LaJeunesse, T.C., 2002. Diversity and community structure of symbiotic dinoflagellates from Caribbean coral reefs. Mar. Biol. 141, 387-400.

LaJeunesse, T.C., 2001. Investigating the biodiversity, ecology, and phylogeny of endosymbiotic dinoflagellates in the genus Symbiodinium using the its region: In search of a "species" level marker. J. Phycol. 37, 866-880. https://doi.org/10.1046/j.1529-8817.2001.01031.x

LaJeunesse, T.C., Loh, W.K.W., Van Woesik, R., Hoegh-Guldberg, O., Schmidt, G.W., Fitt, W.K., 2003. Low symbiont diversity in southern Great Barrier Reef corals, relative to those of the Caribbean. Limnol. Oceanogr. 2046-2054.

LaJeunesse, T.C., Parkinson, J.E., Gabrielson, P.W., Jeong, H.J., Reimer, J.D., Voolstra, C.R., Santos, S.R., 2018. Systematic Revision of Symbiodiniaceae Highlights the Antiquity and Diversity of Coral Endosymbionts. Curr. Biol. 28, 2570-2580.e6. https://doi.org/10.1016/j.cub.2018.07.008

LaJeunesse, T.C., Pettay, D.T., Sampayo, E.M., Phongsuwan, N., Brown, B., Obura, D.O., Hoegh-Guldberg, O., Fitt, W.K., 2010. Long-standing environmental conditions, geographic isolation and host-symbiont specificity influence the relative ecological dominance and genetic diversification of coral endosymbionts in the genus Symbiodinium. J. Biogeogr. 37, 785-800. https://doi.org/10.1111/j.1365-2699.2010.02273.x

LaJeunesse, T.C., Smith, R.T., Finney, J., Oxenford, H., 2009. Outbreak and persistence of opportunistic symbiotic dinoflagellates during the 2005 Caribbean mass coral 'bleaching'event. Proc. R. Soc. London B Biol. Sci. RSPB20091405.

LaJeunesse, T.C., Thornhill, D.J., 2011. Improved resolution of reef-coral endosymbiont (Symbiodinium) species diversity, ecology, and evolution through psbA non-coding region genotyping. PLoS One 6, e29013. https://doi.org/10.1371/journal.pone.0029013

LaJeunesse, T.C., Thornhill, D.J., Cox, E.F., Stanton, F.G., Fitt, W.K., Schmidt, G.W., 2004. High diversity and host specificity observed among symbiotic dinoflagellates in reef coral communities from Hawaii. Coral Reefs 23, 596603. https://doi.org/10.1007/s00338-004-0428-4

LaJeunesse, T.C., Wham, D.C., Pettay, D.T., Parkinson, J.E., Keshavmurthy, S., Chen, C.A., 2014. Ecologically differentiated stress-tolerant endosymbionts in the dinoflagellate genus Symbiodinium (Dinophyceae) Clade D are different species. Phycologia 53, 305-319. https://doi.org/doi:10.2216/13-186.1

LaJeunesse, T.C., Wiedenmann, J., Casado-Amezua, P., D’Ambra, I., E., T.K., Nitschke M. R., Oakley, C.A., Goffredo S, Spano, C.A., Cubillos, V.M., Davy, S.K., Suggett, D.J., Casado-Amezúa, P., D’Ambra, I., Turnham, K.E., Nitschke, M.R., Oakley, C.A., Goffredo, S., Spano, C.A., Cubillos, V.M., 2021. Revival of Philozoon Geddes for hostspecialized dinoflagellates, 'zooxanthellae', in animals from coastal temperate zones of northern and southern hemispheres. Eur. J. Phycol. 1-15. https://doi.org/DOI: 10.1080/09670262.2021.1914863.

Lamoureux, G., Javelle, A., Baday, S., Wang, S., Bernèche, S., 2010. Transport mechanisms in the ammonium transporter family. Transfus. Clin. Biol. 17, 168-175. https://doi.org/10.1016/j.tracli.2010.06.004

Lauritano, C., De Luca, D., Amoroso, M., Benfatto, S., Maestri, S., Racioppi, C., Esposito, F., Ianora, A., 2019. New molecular insights on the response of the green alga Tetraselmis suecica to nitrogen starvation. Sci. Rep. 9. https://doi.org/10.1038/s41598-019-39860-5

Leggat, W., Hoegh-Guldberg, O., Dove, S., Yellowlees, D., 2007. Analysis of an EST library from the dinoflagellate (Symbiodinium sp.) symbiont of reef-building corals. J. Phycol. 43, 1010-1021. https://doi.org/10.1111/j.15298817.2007.00387.x

Lehnert, E.M., Burriesci, M.S., Pringle, J.R., 2012. Developing the anemone Aiptasia as a tractable model for cnidariandinoflagellate symbiosis: the transcriptome of aposymbiotic A. pallida. BMC Genomics 13, 1-10. https://doi.org/10.1186/1471-2164-13-271 
Lehnert, E.M., Mouchka, M.E., Burriesci, M.S., Gallo, N.D., Schwarz, J.A., Pringle, J.R., 2014. Extensive differences in gene expression between symbiotic and aposymbiotic cnidarians. G3 Genes, Genomes, Genet. 4, 277-295. https://doi.org/10.1534/g3.113.009084

Lesser, M.P., Stat, M., Gates, R.D., 2013. The endosymbiotic dinoflagellates (Symbiodinium sp.) of corals are parasites and mutualists. Coral Reefs 32, 603-611.

Levitan, D.R., Boudreau, W., Jara, J., Knowlton, N., 2014. Long-term reduced spawning in Orbicella coral species due to temperature stress. Mar. Ecol. Prog. Ser. 515, 1-10.

Lewis, C.L., Coffroth, M.A., 2004. The Acquisition of Exogenous Algal Symbionts by an Octocoral After Bleaching. Science (80-. ). 304, 1490-1492. https://doi.org/10.1126/science.1097323

Li, C., Gui, S., Yang, T., Walk, T., Wang, X., Liao, H., 2012. Identification of soybean purple acid phosphatase genes and their expression responses to phosphorus availability and symbiosis. Ann. Bot. 109, 275-285. https://doi.org/10.1093/aob/mcr246

Liao, S., Gan, L., Lv, L., Mei, Z., 2020. The regulatory roles of aquaporins in the digestive system. Genes Dis. https://doi.org/10.1016/j.gendis.2019.12.011

Lin, K.L., Wang, J.T., Fang, L.S., 2000. Participation of glycoproteins on zooxanthellal cell walls in the establishment of a symbiotic relationship with the sea anemone, Aiptasia pulchella. Zool. Stud. 39, 172-178.

Lin, Z., Wang, L., Chen, M., Chen, J., 2018. The acute transcriptomic response of coral-algae interactions to pH fluctuation. Mar. Genomics 42, 32-40. https://doi.org/https://doi.org/10.1016/j.margen.2018.08.006

Lipschultz, F., Cook, C.B., 2002. Uptake and assimilation of $15 \mathrm{~N}$-ammonium by the symbiotic sea anemones Bartholomea annulata and Aiptasia pallida: Conservation versus recycling of nitrogen. Mar. Biol. 140, 489-502. https://doi.org/10.1007/s00227-001-0717-1

Little, A.F., van Oppen, M.J.H., Willis, B.L., 2004. Flexibility in Algal Endosymbioses Shapes Growth in Reef Corals. Science (80-. ). 304, 1492-1494. https://doi.org/10.1126/science.1095733

Lodish, H., Berk, A., Kaiser, C.A., Amon, A., Ploegh, H., Bretscher, A., Krieger, M., Martin, K.C., 2016. Molecular Cell Biology, Biochemical Education. Macmillan Learning.

Logan, D.D.K., LaFlamme, A.C., Weis, V.M., Davy, S.K., 2010. Flow-cytometric characterization of the cell-surface glycans of symbiotic dinoflagellates (symbiodinium spp.). J. Phycol. 46, 525-533. https://doi.org/10.1111/j.15298817.2010.00819.x

Loh, W.K.W., Loi, T., Carter, D., Hoegh-Guldberg, O., 2001. Genetic variability of the symbiotic dinoflagellates from the wide ranging coral species Seriatopora hystrix and Acropora longicyathus in the Indo-West Pacific. Mar. Ecol. Prog. Ser. 222, 97-107. https://doi.org/10.3354/meps222097

Loram, J.E., Trapido-Rosenthal, H.G., Douglas, A.E., 2007. Functional significance of genetically different symbiotic algae Symbiodinium in a coral reef symbiosis. Mol. Ecol. 16, 4849-4857.

Maeda, N., Funahashi, T., Hibuse, T., Nagasawa, A., Kishida, K., Kuriyama, H., Nakamura, T., Kihara, S., Shimomura, I., Matsuzawa, Y., 2004. Adaptation to fasting by glycerol transport through aquaporin 7 in adipose tissue. Proc. Natl. Acad. Sci. U. S. A. 101, 17801-17806. https://doi.org/10.1073/pnas.0406230101

Manjithaya, R., Anjard, C., Loomis, W.F., Subramani, S., 2010. Unconventional secretion of Pichia pastoris Acb1 is dependent on GRASP protein, peroxisomal functions, and autophagosome formation. J. Cell Biol. 188, 537-546. https://doi.org/10.1083/jcb.200911149

Maor-Landaw, K., van Oppen, M.J.H., McFadden, G.I., 2020. Symbiotic lifestyle triggers drastic changes in the gene expression of the algal endosymbiont Breviolum minutum (Symbiodiniaceae). Ecol. Evol. 10, 451-466. https://doi.org/10.1002/ece3.5910

Margulis, L., 2013. The Symbiotic Planet: A New Look At Evolution, Science Masters. Orion Publishing Group,.

Markell, D., Trench, R., Iglesias-prieto, R., 1992. Macromolecules associated with the cell walls of symbiotic dinoflagellates. Symbiosis (Philadelphia, PA) 12, 19-31.

Markell, D.A., Wood-Charlson, E.M., 2010. Immunocytochemical evidence that symbiotic algae secrete potential recognition signal molecules in hospite. Mar. Biol. 157, 1105-1111. https://doi.org/10.1007/s00227-010-1392-x

Marshall, P., Schuttenberg, H., 2006. A Reef Manager's Guide to Coral Bleaching. Great Barrier Reef Marine Park Authority, Townsville, Australia. 
Marx, V., 2013. Targeted proteomics. Nat. Methods 10, 19-22. https://doi.org/10.1038/nmeth.2285

Mason, K.A., Johnson, G.B., Losos, J.B., Singer, S., 2017. Understanding Biology. McGraw-Hill Education.

Matthews, J.L., Crowder, C.M., Oakley, C.A., Lutz, A., Roessner, U., Meyer, E., Grossman, A.R., Weis, V.M., Davy, S.K., 2017. Optimal nutrient exchange and immune responses operate in partner specificity in the cnidarian-dinoflagellate symbiosis. Proc. Natl. Acad. Sci. U. S. A. 114, 13194-13199. https://doi.org/10.1073/pnas.1710733114

Matthews, J.L., Oakley, C.A., Lutz, A., Hillyer, K.E., Roessner, U., Grossman, A.R., Weis, V.M., Davy, S.K., 2018. Partner switching and metabolic flux in a model cnidarian-dinoflagellate symbiosis. Proc. R. Soc. B Biol. Sci. 285, 20182336. https://doi.org/10.1098/rspb.2018.2336

Matthews, J.L., Raina, J.B., Kahlke, T., Seymour, J.R., van Oppen, M.J.H., Suggett, D.J., 2020. Symbiodiniaceae-bacteria interactions: rethinking metabolite exchange in reef-building corals as multi-partner metabolic networks. Environ. Microbiol. 22, 1675-1687. https://doi.org/10.1111/1462-2920.14918

Matthews, J.L., Sproles, A.E., Oakley, C.A., Grossman, A.R., Weis, V.M., Davy, S.K., 2016. Menthol-induced bleaching rapidly and effectively provides experimental aposymbiotic sea anemones (Aiptasia sp.) for symbiosis investigations. J. Exp. Biol. 219, 306-310.

McAuley, P.J., Cook, C.B., 1994. Effects of host feeding and dissolved ammonium on cell division and nitrogen status of zooxanthellae in the hydroid Myrionema amboinense. Mar. Biol. 121, 343-348. https://doi.org/10.1007/BF00346743

McCook, L., Folke, C., Hughes, T., Nystrom, M., Obura, D., Salm, R., 2009. Climate Change and the Great Barrier Reef A Vulnerability Assessment- chapter 4. Great Barrier Reef Marine Park Authority and the Australian Greenhouse Office.

McDermott, A.M., Blanquet, R.S., 1991. Glucose and glycerol uptake by isolated zooxanthellae from Cassiopea xamachana: Transport mechanisms and regulation by host homogenate fractions. Mar. Biol. 108, 129-136. https://doi.org/10.1007/BF01313480

McGinty, E.S., Pieczonka, J., Mydlarz, L.D., 2012. Variations in Reactive Oxygen Release and Antioxidant Activity in Multiple Symbiodinium Types in Response to Elevated Temperature. Microb. Ecol. 64, 1000-1007. https://doi.org/10.1007/s00248-012-0085-z

McIlroy, S.E., Gillette, P., Cunning, R., Klueter, A., Capo, T., Baker, A.C., Coffroth, M.A., 2016. The effects of Symbiodinium (Pyrrhophyta) identity on growth, survivorship, and thermal tolerance of newly settled coral recruits. J. Phycol. 52, 1114-1124. https://doi.org/10.1111/jpy.12471

McIlroy, S.E., Wong, J.C.Y., Baker, D.M., 2020. Competitive traits of coral symbionts may alter the structure and function of the microbiome. ISME J. 14, 2424-2432. https://doi.org/10.1038/s41396-020-0697-0

Metsalu, T., Vilo, J., 2015. ClustVis: A web tool for visualizing clustering of multivariate data using Principal Component Analysis and heatmap. Nucleic Acids Res. 43, W566-W570. https://doi.org/10.1093/nar/gkv468

Meyer, E., Weis, V.M., 2012. Study of cnidarian-algal symbiosis in the "omics" age. Biol. Bull. 223, 44-65. https://doi.org/10.1086/BBLv223n1p44

Mies, M., Voolstra, C.R., Castro, C.B., Pires, D.O., Calderon, E.N., Sumida, P.Y.G., 2017. Expression of a symbiosisspecific gene in Symbiodinium type A1 associated with coral, nudibranch and giant clam larvae. R. Soc. Open Sci. 4, 170253. https://doi.org/10.1098/rsos.170253

Miller, D.J., Yellowlees, D., 1989. Inorganic nitrogen uptake by symbiotic marine cnidarians: a critical review. Proc. - R. Soc. London, B 237, 109-125. https://doi.org/10.1098/rspb.1989.0040

Miura, K., Sladoje, N., 2019. Bioimage Data Analysis Workflows. Saint Philip Street Press.

Mohamed, A.R., Cumbo, V., Harii, S., Shinzato, C., Chan, C.X., Ragan, M.A., Bourne, D.G., Willis, B.L., Ball, E.E., Satoh, N., Miller, D.J., 2016. The transcriptomic response of the coral Acropora digitifera to a competent Symbiodinium strain: The symbiosome as an arrested early phagosome. Mol. Ecol. 25, 3127-3141. https://doi.org/10.1111/mec.13659

Mohammed, H., Taylor, C., Brown, G.D., Papachristou, E.K., Carroll, J.S., D’Santos, C.S., 2016. Rapid immunoprecipitation mass spectrometry of endogenous proteins (RIME) for analysis of chromatin complexes. Nat. Protoc. 11, 316-326. https://doi.org/10.1038/nprot.2016.020

Moran, N.A., 2007. Symbiosis as an adaptive process and source of phenotypic complexity. Light Evol. 1, 165-181. https://doi.org/10.17226/11790

Mouyna, I., Hartl, L., Latgé, J.P., 2013. $\beta$-1,3-glucan modifying enzymes in Aspergillus fumigatus. Front. Microbiol. 
https://doi.org/10.3389/fmicb.2013.00081

Mouyna, I., Morelle, W., Vai, M., Monod, M., Léchenne, B., Fontaine, T., Beauvais, A., Sarfati, J., Prévost, M.-C., Henry, C., Latgé, J.-P., 2005. Deletion of GEL2 encoding for a $\beta(1-3)$ glucanosyltransferase affects morphogenesis and virulence in Aspergillus fumigatus. Mol. Microbiol. 56, 1675-1688. https://doi.org/https://doi.org/10.1111/j.13652958.2005.04654.x

Mueckler, M., Thorens, B., 2013. The SLC2 (GLUT) family of membrane transporters. Mol. Aspects Med. 34, 121-138. https://doi.org/10.1016/j.mam.2012.07.001

Mukherjee, P.K., Buensanteai, N., Moran-Diez, M.E., Druzhinina, I.S., Kenerley, C.M., 2012. Functional analysis of nonribosomal peptide synthetases (NRPSs) in Trichoderma virens reveals a polyketide synthase (PKS)/NRPS hybrid enzyme involved in the induced systemic resistance response in maize. Microbiology 158, 155-165. https://doi.org/10.1099/mic.0.052159-0

Muller-Parker, G., Hoegh-Guldberg, O., 1994. Effect of Ammonium Enrichment on Animal and Algal Biomass of the Coral Pocillopora damicornis. Pacific Sci. 48, 273-283.

Muller-parker, G., Lee, W., Cook, C.B., 1996. Changes in the ultrastructure of symbiotic zooxanthellae (Symbiodinium sp., Dinophyceae) in fed and starved sea anemones maintained under high and low light. Cell 994.

Muscatine, L., 1967. Glycerol Excretion by Symbiotic Algae from Corals and Tridacna and Its Control by the Host. Science (80-. ). 156, 516-519. https://doi.org/10.1126/science.156.3774.516

Muscatine, L., 1965. Symbiosis of hydra and algae-III. Extracellular products of the algae. Comp. Biochem. Physiol. 16, 7792. https://doi.org/10.1016/0010-406x(65)90165-9

Muscatine, L., Cernichiari, E., 1969. Assimilation of photosynthetic products of zooxanthellae by a reef coral. Biol. Bull. $137,506-523$.

Muscatine, L., Falkowski, P.G., Dubinsky, Z., 1983. Carbon budgets in symbiotic associations BT - Endocytobiology II. Intracellular Space as Oligogenetic Ecosystem, in: Schenk, H.E.A., Schwemmler, W. (Eds.), . Walter de Gruyter, Berlin and New York.

Muscatine, L., Ferrier-Pagès, C., Blackburn, A., Gates, R.D., Baghdasarian, G., Allemand, D., 1998. Cell-specific density of symbiotic dinoflagellates in tropical anthozoans. Coral Reefs 17, 329-337. https://doi.org/10.1007/s003380050133

Muscatine, L., Hand, C., 1958. Direct Evidence for the Transfer of Materials From Symbiotic Algae To the Tissues of a Coelenterate. Proc. Natl. Acad. Sci. 44, 1259-1263. https://doi.org/10.1073/pnas.44.12.1259

Muscatine, L., Pool, R.R., 1979. Regulation of numbers of intracellular algae. Proc. R. Soc. London - Biol. Sci. 204, 131139. https://doi.org/10.1098/rspb.1979.0018

Muscatine, L., Porter, J.W., 1977. Reef corals: mutualistic symbioses adapted to nutrient-poor environments. Bioscience 27, 454-460.

Nagao, R., Yokono, M., Akimoto, S., Tomo, T., 2013. High Excitation Energy Quenching in Fucoxanthin Chlorophyll a/cBinding Protein Complexes from the Diatom Chaetoceros gracilis. J. Phys. Chem. B 117, 6888-6895. https://doi.org/10.1021/jp403923q

Nakamura, H., Kawase, Y., Maruyama, K., Murai, A., 1998. Studies on Polyketide Metabolites of a Symbiotic Dinoflagellate, Symbiodinium sp.: A New C30 Marine Alkaloid, Zooxanthellamine, a Plausible Precursor for Zoanthid Alkaloids. Bull. Chem. Soc. Jpn. 71, 781-787. https://doi.org/10.1246/bcsj.71.781

Nakamura, J., Straub, K., Wu, J., Lou, L., 1995. The glutamine hydrolysis function of human GMP synthetase. Identification of an essential active site cysteine. J. Biol. Chem. 270, 23450-23455. https://doi.org/10.1074/jbc.270.40.23450

Nandakumar, R., Wakayama, M., Nagano, Y., Kawamura, T., Sakai, K., Moriguchi, M., 1999. Overexpression of salttolerant glutaminase from Micrococcus luteus K-3 in Escherichia coli and its purification. Protein Expr. Purif. 15, 155-161. https://doi.org/10.1006/prep.1998.1005

Navarro-Muñoz, J.C., Collemare, J., 2020. Evolutionary Histories of Type III Polyketide Synthases in Fungi. Front. Microbiol. 10, 1-18. https://doi.org/10.3389/fmicb.2019.03018

Neckelmann, N., Muscatine, L., 1983. Regulatory mechanisms maintaining the Hydra-Chlorella symbiosis . Proc. R. Soc. London. Ser. B. Biol. Sci. 219, 193-210. https://doi.org/10.1098/rspb.1983.0067

Nesvizhskii, A.I., 2014. Proteogenomics: Concepts, applications and computational strategies. Nat. Methods 11, 1114-1125. https://doi.org/10.1038/NMETH.3144 
Neubauer, E.F., Poole, A.Z., Weis, V.M., Davy, S.K., 2016. The scavenger receptor repertoire in six cnidarian species and its putative role in cnidarian-dinoflagellate symbiosis. PeerJ 2016, e2692. https://doi.org/10.7717/peerj.2692

Newsholme, P., Procopio, J., Ramos Lima, M.M., Pithon-Curi, T.C., Curi, R., 2003. Glutamine and glutamate - Their central role in cell metabolism and function. Cell Biochem. Funct. 21, 1-9. https://doi.org/10.1002/cbf.1003

Nickelsen, K., 2015. The Path of Carbon in Photosynthesis (1937-1954), in: History, Philosophy and Theory of the Life Sciences. Springer, pp. 201-250. https://doi.org/10.1007/978-94-017-9582-1_6

Nitschke, M.R., Craveiro, S.C., Brandão, C., Fidalgo, C., Serôdio, J., Calado, A.J., Frommlet, J.C., 2020. Description of Freudenthalidium gen. nov. and Halluxium gen. nov. to Formally Recognize Clades Fr3 and $\mathrm{H}$ as Genera in the Family Symbiodiniaceae (Dinophyceae). J. Phycol. 56, 923-940. https://doi.org/10.1111/jpy.12999

Norman, B.M., Reynolds, S.D., Morgan, D.L., 2021. Three-way symbiotic relationships in whale sharks. Pacific Conserv. Biol. https://doi.org/10.1071/pc20043

Nutt, A., Sild, V., Pettersson, G., Johansson, G., 1998. A mean for functional classification of cellulases. Eur. J. Biochem. 258, 200-206. https://doi.org/10.1046/j.1432-1327.1998.2580200.x

Nyholm, S. V, McFall-Ngai, M., 2004. The winnowing: establishing the squid-vibrio symbiosis. Nat. Rev. Microbiol. 2, 632.

O'Rourke, A.E., Rourke, A.E.O., 2011. Evaluating the transcriptome of the settled polyp of the robust coral, Montastraea faveolata when infected with the competent symbiont, by microarray analysis.

Oakley, C.A., Ameismeier, M.F., Peng, L., Weis, V.M., Grossman, A.R., Davy, S.K., 2016. Symbiosis induces widespread changes in the proteome of the model cnidarian Aiptasia. Cell. Microbiol. 18, 1009-1023. https://doi.org/10.1111/cmi.12564

Oakley, C.A., Durand, E., Wilkinson, S.P., Peng, L., Weis, V.M., Grossman, A.R., Davy, S.K., 2017. Thermal Shock Induces Host Proteostasis Disruption and Endoplasmic Reticulum Stress in the Model Symbiotic Cnidarian Aiptasia. J. Proteome Res. 16, 2121-2134. https://doi.org/10.1021/acs.jproteome.6b00797

Paracer, S., Ahmadjian, V., 1986. Symbiosis. An Introduction to Biological Associations. Bryologist. https://doi.org/10.2307/3243209

Park, J.H., Saier, M.H., 1996. Phylogenetic characterization of the MIP family of transmembrane channel proteins. J. Membr. Biol. 153, 171-180. https://doi.org/10.1007/s002329900120

Parkinson, J.E., Baker, A.C., Baums, I.B., Davies, S.W., Grottoli, A.G., Kitchen, S.A., Matz, M. V., Miller, M.W., Shantz, A.A., Kenkel, C.D., 2019. Molecular tools for coral reef restoration: Beyond biomarker discovery. Conserv. Lett. https://doi.org/10.1111/conl.12687

Parkinson, J.E., Baumgarten, S., Michell, C.T., Baums, I.B., LaJeunesse, T.C., Voolstra, C.R., 2016. Gene expression variation resolves species and individual strains among coral-associated dinoflagellates within the genus symbiodinium. Genome Biol. Evol. 8, 665-680. https://doi.org/10.1093/gbe/evw019

Parkinson, J.E., Tivey, T.R., Mandelare, P.E., Adpressa, D.A., Loesgen, S., Weis, V.M., 2018. Subtle differences in symbiont cell surface glycan profiles do not explain species-specific colonization rates in a model cnidarian-algal symbiosis. Front. Microbiol. https://doi.org/10.3389/fmicb.2018.00842

Pasaribu, B., Weng, L.-C., Lin, I.-P., Camargo, E., Tzen, J.T.C., Tsai, C.-H., Ho, S.-L., Lin, M.-R., Wang, L.-H., Chen, C.S., Jiang, P.-L., 2015. Morphological Variability and Distinct Protein Profiles of Cultured and Endosymbiotic Symbiodinium cells Isolated from Exaiptasia pulchella. Sci. Rep. 5, 15353. https://doi.org/10.1038/srep15353

Patey, M.D., Rijkenberg, M.J.A., Statham, P.J., Stinchcombe, M.C., Achterberg, E.P., Mowlem, M., 2008. Determination of nitrate and phosphate in seawater at nanomolar concentrations. TrAC - Trends Anal. Chem. 27, 169-182. https://doi.org/10.1016/j.trac.2007.12.006

Pawlowski, J.A.N., Holzmann, M., Fahrni, J.F., Pochon, X., Lee, J.J., 2001. Molecular Identification of Algal Endosymbionts in Large Miliolid Foraminifera: 2. Dinoflagellates. J. Eukaryot. Microbiol. 48, 368-373. https://doi.org/10.1111/j.1550-7408.2001.tb00326.x

Paxton, C.W., Davy, S.K., Weis, V.M., 2013. Stress and death of cnidarian host cells play a role in cnidarian bleaching. J. Exp. Biol. 216, 2813-2820. https://doi.org/10.1242/jeb.087858

Peng, S.E., Wang, Y.B., Wang, L.H., Chen, W.N.U., Lu, C.Y., Fang, L.S., Chen, C.S., 2010. Proteomic analysis of symbiosome membranes in cnidaria - Dinoflagellate endosymbiosis. Proteomics 10, 1002-1016. https://doi.org/10.1002/pmic.200900595 
Penheiter, A.R., Duff, S.M.G., Sarath, G., 1997. Soybean Root Nodule Acid Phosphatase. Plant Physiol. 114, 597 LP - 604. https://doi.org/10.1104/pp.114.2.597

Perez, S., Weis, V., 2008. Cyclophilin and the regulation of symbiosis in Aiptasia pallida. Biol. Bull. 215, 63-72. https://doi.org/10.2307/25470684

Pernice, M., Dunn, S.R., Tonk, L., Dove, S., Domart-Coulon, I., Hoppe, P., Schintlmeister, A., Wagner, M., Meibom, A., 2015. A nanoscale secondary ion mass spectrometry study of dinoflagellate functional diversity in reef-building corals. Environ. Microbiol. 17, 3570-3580.

Pernice, M., Meibom, A., Van Den Heuvel, A., Kopp, C., Domart-Coulon, I., Hoegh-Guldberg, O., Dove, S., 2012. A single-cell view of ammonium assimilation in coral-dinoflagellate symbiosis microbe-microbe and microbe-host interactions. ISME J. 6, 1314-1324.

Pettay, D., Whama, D.C., Smith, R.T., Iglesias-Prietoc, R., LaJeunessea, T.C., 2015. Microbial invasion of the Caribbean by an Indo-Pacific coral zooxanthella. Proc. Natl. Acad. Sci. U. S. A. 112, 7513-7518. https://doi.org/10.1073/pnas.1502283112

Pike, J.A., Styles, I.B., Rappoport, J.Z., Heath, J.K., 2017. Quantifying receptor trafficking and colocalization with confocal microscopy. Methods 115, 42-54. https://doi.org/10.1016/j.ymeth.2017.01.005

Pizzagalli, M.D., Bensimon, A., Superti-Furga, G., 2021. A guide to plasma membrane solute carrier proteins. FEBS J. 288, 2784-2835. https://doi.org/10.1111/febs.15531

Pochon, X., Gates, R.D., 2010. A new Symbiodinium clade (Dinophyceae) from soritid foraminifera in Hawai'i. Mol. Phylogenet. Evol. 56, 492-497. https://doi.org/10.1016/j.ympev.2010.03.040

Pochon, X., LaJeunesse, T.C., 2021. Miliolidium n. gen, a New Symbiodiniacean Genus Whose Members Associate with Soritid Foraminifera or Are Free-Living . J. Eukaryot. Microbiol. 1-9. https://doi.org/10.1111/jeu.12856

Pochon, X., Pawlowski, J., Zaninetti, L., Rowan, R., 2001. High genetic diversity and relative specificity among Symbiodinium-like endosymbiotic dinoflagellates in soritid foraminiferans. Mar. Biol. 139, 1069-1078. https://doi.org/10.1007/s002270100674

Polovina, J.J., 1984. Model of a coral reef ecosystem. Coral Reefs 3, 1-11. https://doi.org/10.1007/BF00306135

Pupier, C.A., Fine, M., Bednarz, V.N., Rottier, C., Grover, R., Ferrier-Pagès, C., 2019. Productivity and carbon fluxes depend on species and symbiont density in soft coral symbioses. Sci. Rep. 9, 1-10.

Putnam, N.H., Srivastava, M., Hellsten, U., Dirks, B., Chapman, J., Salamov, A., Terry, A., Shapiro, H., Lindquist, E., Kapitonov, V. V., Jurka, J., Genikhovich, G., Grigoriev, I. V., Lucas, S.M., Steele, R.E., Finnerty, J.R., Technau, U., Martindale, M.Q., Rokhsar, D.S., 2007. Sea anemone genome reveals ancestral eumetazoan gene repertoire and genomic organization. Science (80-. ). 317, 86-94. https://doi.org/10.1126/science.1139158

Qiao, K., Wang, M., Takano, T., Liu, S., 2018. Overexpression of acyl-coa-binding protein 1 (ChACBP1) from saline-alkalitolerant chlorella sp. Enhances stress tolerance in arabidopsis. Front. Plant Sci. https://doi.org/10.3389/fpls.2018.01772

R Core Team, 2020. R: A Language and Environment for Statistical Computing. R Foundation for Statistical Computing.

Rädecker, N., Pogoreutz, C., Wild, C., Voolstra, C.R., 2017a. Stimulated respiration and net photosynthesis in Cassiopeia sp. during glucose enrichment suggests in hospite $\mathrm{CO} 2$ limitation of algal endosymbionts. Front. Mar. Sci. https://doi.org/10.3389/fmars.2017.00267

Rädecker, N., Raina, J.B., Pernice, M., Perna, G., Guagliardo, P., Kilburn, M.R., Aranda, M., Voolstra, C.R., 2017 b. Aiptasia as a model to study metabolic diversity and specificity in cnidarian-dinoflagellate symbioses. bioRxiv. https://doi.org/10.1101/223933

Rahav, O., Dubinsky, Z., Achituv, Y., Falkowski, P.G., 1989. Ammonium metabolism in the zooxanthellate coral, stylophora pistillata . Proc. R. Soc. London. B. Biol. Sci. 236, 325-337. https://doi.org/10.1098/rspb.1989.0026

Rands, M.L., Loughman, B.C., Douglas, A.E., 1993. The symbiotic interface in an alga-invertebrate symbiosis. Proc. R. Soc. B Biol. Sci. 253, 161-165. https://doi.org/10.1098/rspb.1993.0097

Raven, J.A., Evans, M.C.W., Korb, R.E., 1999. The role of trace metals in photosynthetic electron transport in O2-evolving organisms. Photosynth. Res. 60, 111-150. https://doi.org/10.1023/a:1006282714942

Reaka-Kudla, M.L., 2001. Known and Unknown Biodiversity, Risk of Extinction and Conservation Strategy in the Sea, in: Bendell-Young, L., Gallaugher, P. (Eds.), Waters in Peril. Springer US, Boston, MA, pp. 19-33. https://doi.org/10.1007/978-1-4615-1493-0_2 
Rehman, A.U., Szabó, M., Deák, Z., Sass, L., Larkum, A., Ralph, P., Vass, I., 2016. Symbiodinium sp. cells produce lightinduced intra- and extracellular singlet oxygen, which mediates photodamage of the photosynthetic apparatus and has the potential to interact with the animal host in coral symbiosis. New Phytol. 212, 472-484. https://doi.org/10.1111/nph.14056

Reich, H.G., Robertson, D.L., Goodbody-Gringley, G., 2017. Do the shuffle: Changes in Symbiodinium consortia throughout juvenile coral development. PLoS One 12, e0171768. https://doi.org/10.1371/journal.pone.0171768

Reich, H.G., Rodriguez, I.B., LaJeunesse, T.C., Ho, T.Y., 2020. Endosymbiotic dinoflagellates pump iron: differences in iron and other trace metal needs among the Symbiodiniaceae. Coral Reefs 39, 915-927. https://doi.org/10.1007/s00338-020-01911-z

Reinbothe, C., Pollmann, S., Reinbothe, S., 2010. Singlet oxygen signaling links photosynthesis to translation and plant growth. Trends Plant Sci. https://doi.org/10.1016/j.tplants.2010.05.011

Renshaw, S., 2017. Immunohistochemistry and immunocytochemistry, in: Immunohistochemistry: Essential Methods. John Wiley \&amp; Sons, Ltd, pp. 35-102. https://doi.org/10.1002/9781118717769.ch3

Reynaud, S., Martinez, P., Houlbrèque, F., Billy, I., Allemand, D., Ferrier-Pagès, C., 2009. Effect of light and feeding on the nitrogen isotopic composition of a zooxanthellate coral: Role of nitrogen recycling. Mar. Ecol. Prog. Ser. 392, 103110. https://doi.org/10.3354/meps08195

Reynolds, J.M.C., Bruns, B.U., Fitt, W.K., Schmidt, G.W., 2008. Enhanced photoprotection pathways in symbiotic dinoflagellates of shallow-water corals and other cnidarians (Proceedings of the National Academy of Sciences of the United States of America (2008) 105, (13674-13678) doi: 10.1073/pnas.0805187105). Proc. Natl. Acad. Sci. U. S. A. 105, 17206. https://doi.org/10.1073/pnas.0809220105

Reynolds, W.S., Schwarz, J.A., Weis, V.M., 2000. Symbiosis-enhanced gene expression in cnidarian-algal associations: Cloning and characterization of a cDNA, sym32, encoding a possible cell adhesion protein. Comp. Biochem. Physiol. - A Mol. Integr. Physiol. 126, 33-44. https://doi.org/10.1016/S0742-8413(00)00099-2

Rice, P.M., Bleasby, A.J., Ison, J.C., Mullan, L., Bottu, G., 2011. EMBOSS User’s Guide: Practical Bioinformatics. Cambridge University Press.

Richier, S., Merle, P.L., Furla, P., Pigozzi, D., Sola, F., Allemand, D., 2003. Characterization of superoxide dismutases in anoxia- and hyperoxia-tolerant symbiotic cnidarians, Biochimica et Biophysica Acta - General Subjects. https://doi.org/10.1016/S0304-4165(03)00049-7

Richier, S., Sabourault, C.C., Courtiade, J., Zucchini, N., Allemand, D., Furla, P., 2006. Oxidative stress and apoptotic events during thermal stress in the symbiotic sea anemone, Anemonia viridis. FEBS J. 273, 4186-4198. https://doi.org/10.1111/j.1742-4658.2006.05414.x

Richmond, R.H., 1993. Coral Reefs: Present Problems and Future Concerns Resulting from Anthropogenic Disturbance. Am. Zool. 33, 524-536. https://doi.org/10.1093/icb/33.6.524

Riegl, B.M., Purkis, S.J., Al-Cibahy, A.S., Al-Harthi, S., Grandcourt, E., Al-Sulaiti, K., Baldwin, J., Abdel-Moati, A.M., 2012. Coral Bleaching and Mortality Thresholds in the SE Gulf: Highest in the World, in: Riegl, B.M., Purkis, S.J. (Eds.), . Springer Netherlands, Dordrecht, pp. 95-105. https://doi.org/10.1007/978-94-007-3008-3_6

Roberts, J.M., Fixter, L.M., Davies, P.S., 2001. Ammonium metabolism in the symbiotic sea anemone Anemonia viridis. Hydrobiologia 461, 25-35. https://doi.org/10.1023/A:1012752828587

Roberty, S., Béraud, E., Grover, R., Ferrier-Pagès, C., 2020. Coral productivity is co-limited by bicarbonate and ammonium availability. Microorganisms 8. https://doi.org/10.3390/microorganisms 8050640

Roberty, S., Furla, P., Plumier, J.C., 2016. Differential antioxidant response between two Symbiodinium species from contrasting environments. Plant Cell Environ. 39, 2713-2724. https://doi.org/10.1111/pce.12825

Robinson, W.D., Park, J., Tran, H.T., Del Vecchio, H.A., Ying, S., Zins, J.L., Patel, K., McKnight, T.D., Plaxton, W.C., 2012. The secreted purple acid phosphatase isozymes AtPAP12 and AtPAP26 play a pivotal role in extracellular phosphate-scavenging by Arabidopsis thaliana. J. Exp. Bot. 63, 6531-6542. https://doi.org/10.1093/jxb/ers309

Rodriguez, I.B., Ho, T.Y., 2018. Trace metal requirements and interactions in Symbiodinium kawagutii. Front. Microbiol. https://doi.org/10.3389/fmicb.2018.00142

Rodriguez, I.B., Lin, S., Ho, J., Ho, T.Y., 2016. Effects of trace metal concentrations on the growth of the coral endosymbiont Symbiodinium kawagutii. Front. Microbiol. https://doi.org/10.3389/fmicb.2016.00082

Rohwer, F., Seguritan, V., Azam, F., Knowlton, N., 2002. Diversity and distribution of coral-associated bacteria. Mar. Ecol. Prog. Ser. 243, 1-10. 
Ros, M., Suggett, D.J., Edmondson, J., Haydon, T., Hughes, D.J., Kim, M., Guagliardo, P., Bougoure, J., Pernice, M., Raina, J.-B., 2021. Symbiont shuffling across environmental gradients aligns with changes in carbon uptake and translocation in the reef-building coral Pocillopora acuta. Coral Reefs 40, 595-607.

Rosenberg, E., Koren, O., Reshef, L., Efrony, R., Zilber-Rosenberg, I., 2007. The role of microorganisms in coral health, disease and evolution. Nat. Rev. Microbiol. 5, 355-362. https://doi.org/10.1038/nrmicro1635

Rosic, N., Ling, E.Y.S., Chan, C.K.K., Lee, H.C., Kaniewska, P., Edwards, D., Dove, S., Hoegh-Guldberg, O., 2015. Unfolding the secrets of coral-Algal symbiosis. ISME J. 9, 844-856. https://doi.org/10.1038/ismej.2014.182

Rosset, S., Koster, G., Brandsma, J., Hunt, A.N., Postle, A.D., D’Angelo, C., 2019. Lipidome analysis of Symbiodiniaceae reveals possible mechanisms of heat stress tolerance in reef coral symbionts. Coral Reefs 38, 1241-1253. https://doi.org/10.1007/s00338-019-01865-x

Rosset, S., Wiedenmann, J., Reed, A.J., D’Angelo, C., 2017. Phosphate deficiency promotes coral bleaching and is reflected by the ultrastructure of symbiotic dinoflagellates. Mar. Pollut. Bull. 118, 180-187. https://doi.org/10.1016/j.marpolbul.2017.02.044

Rosset, S.L., Oakley, C.A., Ferrier-Pagès, C., Suggett, D.J., Weis, V.M., Davy, S.K., 2021. The Molecular Language of the Cnidarian-Dinoflagellate Symbiosis. Trends Microbiol. 29, 320-333. https://doi.org/10.1016/j.tim.2020.08.005

Rowan, R., Knowlton, N., 1995. Intraspecific diversity and ecological zonation in coral-algal symbiosis. Proc. Natl. Acad. Sci. 92, 2850-2853.

Rowan, R., Knowlton, N., Baker, A., Jara, J., 1997. Landscape ecology of algal symbionts creates variation in episodes of coral bleaching. Nature 388, 265-269.

Rowan, R., Powers, D., 1991a. Molecular genetic identification of symbiotic dinoflagellates (zooxanthellae). Mar. Ecol. Prog. Ser. 71, 65-73. https://doi.org/10.3354/meps071065

Rowan, R., Powers, D.A., 1991b. A molecular genetic classification of zooxanthellae and the evolution of animal-algal symbioses. Science (80-. ). 251, 1348-1351. https://doi.org/10.1126/science.251.4999.1348

Rudkouskaya, A., Sinsuebphon, N., Ward, J., Tubbesing, K., Intes, X., Barroso, M., 2018. Quantitative imaging of receptorligand engagement in intact live animals. J. Control. Release 286, 451-459. https://doi.org/10.1016/j.jconrel.2018.07.032

Rueden, C.T., Schindelin, J., Hiner, M.C., DeZonia, B.E., Walter, A.E., Arena, E.T., Eliceiri, K.W., 2017. ImageJ2: ImageJ for the next generation of scientific image data. BMC Bioinformatics 18, 529. https://doi.org/10.1186/s12859-0171934-z

Ruhland, A., Leal, N., Kima, P.E., 2007. Leishmania promastigotes activate PI3K/Akt signalling to confer host cell resistance to apoptosis. Cell. Microbiol. 9, 84-96.

Rungroj, N., Devonald, M.A.J., Cuthbert, A.W., Reimann, F., Akkarapatumwong, V., Yenchitsomanus, P.T., Bennett, W.M., Karet, F.E., 2004. A Novel Missense Mutation in AE1 Causing Autosomal Dominant Distal Renal Tubular Acidosis Retains Normal Transport Function but Is Mistargeted in Polarized Epithelial Cells. J. Biol. Chem. 279, 1383313838. https://doi.org/10.1074/jbc.M400188200

Sadava, D., Hillis, D., Heller, C., Berenbaum, M., 2011. Life: The Science of Biology, The science of biology., Life: The Science of Biology. W. H. Freeman. https://doi.org/10.5962/bhl.title.5871

Sampayo, E.M., Ridgway, T., Franceschinis, L., Roff, G., Hoegh-Guldberg, O., Dove, S., 2016. Coral symbioses under prolonged environmental change: living near tolerance range limits. Sci. Rep. 6, 1-12.

Santos, S.R., Shearer, T.L., Hannes, A.R., Coffroth, M.A., 2004. Fine-scale diversity and specificity in the most prevalent lineage of symbiotic dinoflagellates (Symbiodinium, Dinophyceae) of the Caribbean. Mol. Ecol. 13, 459-469. https://doi.org/10.1046/j.1365-294X.2003.02058.x

Saraf, A., Cervantes, S., Bunnik, E.M., Ponts, N., Sardiu, M.E., Chung, D.W.D., Prudhomme, J., Varberg, J.M., Wen, Z., Washburn, M.P., Florens, L., Le Roch, K.G., 2016. Dynamic and combinatorial landscape of histone modifications during the intraerythrocytic developmental cycle of the malaria parasite. J. Proteome Res. 15, 2787-2801. https://doi.org/10.1021/acs.jproteome.6b00366

Sasikumar, A.N., Perez, W.B., Kinzy, T.G., 2012. The many roles of the eukaryotic elongation factor 1 complex. Wiley Interdiscip. Rev. RNA 3, 543-555. https://doi.org/10.1002/wrna.1118

Schindelin, J., Arganda-Carreras, I., Frise, E., Kaynig, V., Longair, M., Pietzsch, T., Preibisch, S., Rueden, C., Saalfeld, S., Schmid, B., Tinevez, J.Y., White, D.J., Hartenstein, V., Eliceiri, K., Tomancak, P., Cardona, A., 2012. Fiji: An opensource platform for biological-image analysis. Nat. Methods 9, 676-682. https://doi.org/10.1038/nmeth.2019 
Schoenberg, D.A., Trench, R.K., Smith, D.C., 1980. Genetic variation in Symbiodinium (= Gymnodinium) microadriaticum Freudenthal, and specificity in its symbiosis with marine invertebrates. III. Specificity and inlfectivity of Symbiodinium microadriaticum. Proc. R. Soc. London. Ser. B. Biol. Sci. 207, 445-460. https://doi.org/10.1098/rspb.1980.0033

Schubert, O.T., Röst, H.L., Collins, B.C., Rosenberger, G., Aebersold, R., 2017. Quantitative proteomics: Challenges and opportunities in basic and applied research. Nat. Protoc. 12, 1289-1294. https://doi.org/10.1038/nprot.2017.040

Schwämmle, V., Hagensen, C.E., Rogowska-Wrzesinska, A., Jensen, O.N., 2020. PolySTest: Robust statistical testing of proteomics data with missing values improves detection of biologically relevant features. Mol. Cell. Proteomics 19, mcp.RA119.001777. https://doi.org/10.1074/mcp.RA119.001777

Schwarz, J.A., Weis, V.M., 2003. Localization of a Symbiosis-Related Protein, Sym32, in the Anthopleura elegantissimaSymbiodinium muscatinei Association. Biol. Bull. 205, 339-350. https://doi.org/10.2307/1543297

Segovia, M., 2008. Programmed cell death in dinoflagellates, in: Programmed Cell Death in Protozoa. Springer, pp. 126142.

Sheppard, C.R.C., Davy, S.K., Pilling, G.M., Graham, N.A.J., 2018. The biology of coral reefs: Second edition, in: The Biology of Coral Reefs: Second Edition. Oxford University Press, Oxford, pp. 1-370. https://doi.org/10.1093/oso/9780198787341.001.0001

Shi, X., Lin, X., Li, L., Li, M., Palenik, B., Lin, S., 2017. Transcriptomic and microRNAomic profiling reveals multi-faceted mechanisms to cope with phosphate stress in a dinoflagellate. ISME J. 11, 2209-2218. https://doi.org/10.1038/ismej.2017.81

Shinzato, C., Inoue, M., Kusakabe, M., 2014. A snapshot of a coral "holobiont": A transcriptome assembly of the scleractinian coral, Porites, captures a wide variety of genes from both the host and symbiotic zooxanthellae. PLoS One 9, e85182. https://doi.org/10.1371/journal.pone.0085182

Silverstein, R.N., Correa, A.M.S., LaJeunesse, T.C., Baker, A.C., 2011. Novel algal symbiont (Symbiodinium spp.) diversity in reef corals of Western Australia. Mar Ecol Prog Ser 422, 63-75.

Silverstein, R.N., Cunning, R., Baker, A.C., 2017. Tenacious D: Symbiodinium in clade D remain in reef corals at both high and low temperature extremes despite impairment. J. Exp. Biol. 220, 1192-1196. https://doi.org/10.1242/jeb.148239

Slaby, B.M., Hackl, T., Horn, H., Bayer, K., Hentschel, U., 2017. Metagenomic binning of a marine sponge microbiome reveals unity in defense but metabolic specialization. ISME J. 11, 2465-2478. https://doi.org/10.1038/ismej.2017.101

Slattery, M., Ankisetty, S., Corrales, J., Marsh-Hunkin, K.E., Gochfeld, D.J., Willett, K.L., Rimoldi, J.M., 2012. Marine proteomics: A critical assessment of an emerging technology. J. Nat. Prod. 75, 1833-1837. https://doi.org/10.1021/np300366a

Smith, G.J., Muscatine, L., 1999. Cell cycle of symbiotic dinoflagellates: Variation in G1 phase-duration with anemone nutritional status and macronutrient supply in the Aiptasia pulchella-Symbiodinium pulchrorum symbiosis. Mar. Biol. 134, 405-418. https://doi.org/10.1007/s002270050557

Smith, S.E., Gianinazzi-Pearson, V., 1988. Physiological Interactions Between Symbionts in Vesicular-Arbuscular Mycorrhizal Plants. Annu. Rev. Plant Physiol. Plant Mol. Biol. 39, 221-244. https://doi.org/10.1146/annurev.pp.39.060188.001253

Sproles, A.E., Kirk, N.L., Kitchen, S.A., Oakley, C.A., Grossman, A.R., Weis, V.M., Davy, S.K., 2018. Phylogenetic characterization of transporter proteins in the cnidarian-dinoflagellate symbiosis. Mol. Phylogenet. Evol. 120, $307-$ 320. https://doi.org/https://doi.org/10.1016/j.ympev.2017.12.007

Sproles, A.E., Oakley, C.A., Krueger, T., Grossman, A.R., Weis, V.M., Meibom, A., Davy, S.K., 2020. Sub-cellular imaging shows reduced photosynthetic carbon and increased nitrogen assimilation by the non-native endosymbiont Durusdinium trenchii in the model cnidarian Aiptasia. Environ. Microbiol. 22, 3741-3753. https://doi.org/10.1111/1462-2920.15142

Sproles, A.E., Oakley, C.A., Matthews, J.L., Peng, L., Owen, J.G., Grossman, A.R., Weis, V.M., Davy, S.K., 2019. Proteomics quantifies protein expression changes in a model cnidarian colonised by a thermally tolerant but suboptimal symbiont. ISME J. https://doi.org/10.1038/s41396-019-0437-5

Starzak, D.E., Quinnell, R.G., Cook, C.B., Davy, S.K., 2020. Influence of Symbiont Species on the Glycerol and Glucose Pools in a Model Cnidarian-Dinoflagellate Symbiosis. Biol. Bull. 239, 143-151.

Starzak, D.E., Quinnell, R.G., Nitschke, M.R., Davy, S.K., 2014. The influence of symbiont type on photosynthetic carbon flux in a model cnidarian-dinoflagellate symbiosis. Mar. Biol. 161, 711-724. https://doi.org/10.1007/s00227-013$2372-8$ 
Stat, M., Carter, D., Hoegh-Guldberg, O., 2006. The evolutionary history of Symbiodinium and scleractinian hostsSymbiosis, diversity, and the effect of climate change. Perspect. Plant Ecol. Evol. Syst. 8, 23-43. https://doi.org/10.1016/j.ppees.2006.04.001

Stat, M., Gates, R.D., 2010. Clade D Symbiodinium in Scleractinian Corals: A "Nugget" of Hope, a Selfish Opportunist, an Ominous Sign, or All of the Above? J. Mar. Biol. 2011, 9. https://doi.org/10.1155/2011/730715

Stat, M., Morris, E., Gates, R.D., 2008. Functional diversity in coral-dinoflagellate symbiosis. Proc. Natl. Acad. Sci. 105, 9256-9261.

Stat, M., Pochon, X., Cowie, R.O.M., Gates, R.D., 2009. Specificity in communities of Symbiodinium in corals from Johnston Atoll. Mar. Ecol. Prog. Ser 386, 83-96.

Stincone, A., Prigione, A., Cramer, T., Wamelink, M.M.C., Campbell, K., Cheung, E., Olin-Sandoval, V., Grüning, N.M., Krüger, A., Tauqeer Alam, M., Keller, M.A., Breitenbach, M., Brindle, K.M., Rabinowitz, J.D., Ralser, M., 2015. The return of metabolism: Biochemistry and physiology of the pentose phosphate pathway. Biol. Rev. 90, 927-963. https://doi.org/10.1111/brv.12140

Stochaj, W.R., Grossman, A.R., 1997. Differences in the protein profiles of cultured and endosymbiotic Symbiodinium sp. (Pyrrophyta) from the anemone Aiptasia pallida (Anthozoa). J. Phycol. 33, 44-53. https://doi.org/10.1111/j.00223646.1997.00044.x

Streamer, M., McNeil, Y.R., Yellowlees, D., 1993. Photosynthetic carbon dioxide fixation in zooxanthellae. Mar. Biol. 115, 195-198. https://doi.org/10.1007/BF00346335

Sturgill-Koszycki, S., Schlesinger, P.H., Chakraborty, P., Haddix, P.L., Collins, H.L., Fok, A.K., Allen, R.D., Gluck, S.L., Heuser, J., Russell, D.G., 1994. Lack of acidification in Mycobacterium phagosomes produced by exclusion of the vesicular proton-ATPase. Science (80-. ). 263, 678-681. https://doi.org/10.1126/science.8303277

Suescún-Bolívar, L.P., Traverse, G.M.I., Thomé, P.E., 2016. Glycerol outflow in Symbiodinium under osmotic and nitrogen stress. Mar. Biol. 163, 128. https://doi.org/10.1007/s00227-016-2899-6

Suggett, D.J., Warner, M.E., Leggat, W., 2017. Symbiotic Dinoflagellate Functional Diversity Mediates Coral Survival under Ecological Crisis. Trends Ecol. Evol. 32, 735-745. https://doi.org/10.1016/j.tree.2017.07.013

Summons, R.E., Boag, T.S., 1986. The effect of ammonium on photosynthesis and the pathway of ammonium assimilation in Gymnodinium microadriaticum in vitro and in symbiosis with tridacnid clams and corals . Proc. R. Soc. London. Ser. B. Biol. Sci. 227, 147-159. https://doi.org/10.1098/rspb.1986.0016

Sunda, W.G., 2012. Feedback interactions between trace metal nutrients and phytoplankton in the ocean. Front. Microbiol. 3 , 204. https://doi.org/10.3389/fmicb.2012.00204

Szabados, L., Savouré, A., 2010. Proline: a multifunctional amino acid. Trends Plant Sci. 15, 89-97. https://doi.org/10.1016/j.tplants.2009.11.009

Takahashi, S., Nakamura, T., Sakamizu, M., Van Woesik, R., Yamasaki, H., 2004. Repair Machinery of Symbiotic Photosynthesis as the Primary Target of Heat Stress for Reef-Building Corals. Plant Cell Physiol. 45, 251-255. https://doi.org/10.1093/pcp/pch028

Tanaka, Y., Miyajima, T., Koike, I., Hayashibara, T., Ogawa, H., 2006. Translocation and conservation of organic nitrogen within the coral-zooxanthella symbiotic system of Acropora pulchra, as demonstrated by dual isotope-labeling techniques. J. Exp. Mar. Bio. Ecol. 336, 110-119. https://doi.org/10.1016/j.jembe.2006.04.011

Taylor, C.E., Muscatine, L., Jefferson, D.R., 1989. Maintenance and breakdown of the Hydra-Chlorella symbiosis: A computer model. Proc. R. Soc. B Biol. Sci. 238, 277-289. https://doi.org/10.1098/rspb.1989.0081

Tcherkez, G., Carroll, A., Abadie, C., Mainguet, S., Davanture, M., Zivy, M., 2020. Protein synthesis increases with photosynthesis via the stimulation of translation initiation. Plant Sci. 291, 110352. https://doi.org/10.1016/j.plantsci.2019.110352

Tchernov, D., Gorbunov, M.Y., de Vargas, C., Yadav, S.N., Milligan, A.J., Häggblom, M., Falkowski, P.G., 2004. Membrane lipids of symbiotic algae are diagnostic of sensitivity to thermal bleaching in corals. Proc. Natl. Acad. Sci. U. S. A. 101, 13531-13535.

Ter Beek, J., Guskov, A., Slotboom, D.J., 2014. Structural diversity of ABC transporters. J. Gen. Physiol. 143, $419-435$. https://doi.org/10.1085/jgp.201411164

Thomas, D., Bron, P., Ranchy, G., Duchesne, L., Cavalier, A., Rolland, J.P., Raguénès-Nicol, C., Hubert, J.F., Haase, W., Delamarche, C., 2002. Aquaglyceroporins, one channel for two molecules. Biochim. Biophys. Acta - Bioenerg. 1555, 181-186. https://doi.org/10.1016/S0005-2728(02)00275-X 
Thornhill, D.J., Daniel, M.W., LaJeunesse, T.C., Schmidt, G.W., Fitt, W.K., 2006. Natural infections of aposymbiotic Cassiopea xamachana scyphistomae from environmental pools of Symbiodinium. J. Exp. Mar. Bio. Ecol. 338, 50-56. https://doi.org/http://dx.doi.org/10.1016/j.jembe.2006.06.032

Thornhill, D.J., Lajeunesse, T.C., Santos, S.R., 2007. Measuring rDNA diversity in eukaryotic microbial systems: how intragenomic variation, pseudogenes, and PCR artifacts confound biodiversity estimates. Mol. Ecol. 16, 5326-5340.

Thornhill, D.J., Lewis, A.M., Wham, D.C., Lajeunesse, T.C., 2014. Host-specialist lineages dominate the adaptive radiation of reef coral endosymbionts. Evolution (N. Y). 68, 352-367. https://doi.org/10.1111/evo.12270

Thornhill, D.J., Xiang, Y., Fitt, W.K., Santos, S.R., 2009. Reef endemism, host specificity and temporal stability in populations of symbiotic dinoflagellates from two ecologically dominant Caribbean corals. PLoS One 4, e6262. https://doi.org/10.1371/journal.pone.0006262

Thornhill, D.J., Xiang, Y., Pettay, D.T., Zhong, M., Santos, S.R., 2013. Population genetic data of a model symbiotic cnidarian system reveal remarkable symbiotic specificity and vectored introductions across ocean basins. Mol. Ecol. 22, 4499-4515. https://doi.org/10.1111/mec.12416

Thrall, P.H., Hochberg, M.E., Burdon, J.J., Bever, J.D., 2007. Coevolution of symbiotic mutualists and parasites in a community context. Trends Ecol. Evol. 22, 120-126. https://doi.org/10.1016/j.tree.2006.11.007

Tiller, K.E., Tessier, P.M., 2015. Advances in Antibody Design. Annu. Rev. Biomed. Eng. 17, 191-216. https://doi.org/10.1146/annurev-bioeng-071114-040733

Timm, T., Annoscia, G., Klein, J., Lochnit, G., 2017. The eukaryotic elongation factor 1 alpha (eEF1a) from the parasite leishmania infantum is modified with the immunomodulatory substituent phosphorylcholine (PC). Molecules 22, 2094. https://doi.org/10.3390/molecules22122094

Titlyanov, E.A., Tsukahara, J., Titlyanova, T. V., Leletkin, V.A., Van Woesik, R., Yamazato, K., 2000. Zooxanthellae population density and physiological state of the coral Stylophora pistillata during starvation and osmotic shock. Symbiosis 28, 303-322.

Tivey, T.R., Parkinson, J.E., Weis, V.M., 2020. Host and Symbiont Cell Cycle Coordination Is Mediated by Symbiotic State, Nutrition, and Partner Identity in a Model Cnidarian-Dinoflagellate Symbiosis. MBio 11, e02626-19. https://doi.org/10.1128/mBio.02626-19

Tomanek, L., 2014. Proteomics to study adaptations in marine organisms to environmental stress. J. Proteomics 105,92 106. https://doi.org/10.1016/j.jprot.2014.04.009

Towbin, H., Gordon, J., 1984. Immunoblotting and dot immunobinding - Current status and outlook. J. Immunol. Methods 72, 313-340. https://doi.org/10.1016/0022-1759(84)90001-2

Trench, R., 1997. Diversity of symbiotic dinoflagellates and the evolution of microalgal-invertebrate symbioses, in: Proceedings of the 8th International Coral Reef Symposium. pp. 1275-1286.

Trench, R.K., 1993. Microalgal-Invertebrate Symbioses - A Review. Endocytobiosis Cell Res. 9, 135-175.

Trench, R.K., 1979. The cell biology of plant-animal symbiosis. Annu. Rev. Plant Physiol. 30, 485-531.

Trench, R.K., 1974. Nutritional potentials in Zoanthus sociathus (Coelenterata, Anthozoa). Helgoländer Wissenschaftliche Meeresuntersuchungen 26, 174-216.

Trench, R.K., 1971. The physiology and biochemistry of zooxanthellae symbiotic with marine coelenterates I. The assimilation of photosynthetic products of zooxanthellae by two marine coelenterates. Proc. R. Soc. London. Ser. B. Biol. Sci. 177, 225-235.

Trench, R.K., Blank, R.J., 1987. Symbiodinium Microadriaticum Freudenthal, S. Goreauii Sp. Nov., S. Kawagutii Sp. Nov. and S. Pilosum Sp. Nov.: Gymnodinioid Dinoflagellate Symbionts of Marine Invertebrates. J. Phycol. 23, 469-481. https://doi.org/10.1111/j.1529-8817.1987.tb02534.x

Tsiatsiani, L., Van Breusegem, F., Gallois, P., Zavialov, A., Lam, E., Bozhkov, P. V., 2011. Metacaspases. Cell Death Differ. 18, 1279-1288. https://doi.org/10.1038/cdd.2011.66

Tyanova, S., Cox, J., 2018. Perseus: A bioinformatics platform for integrative analysis of proteomics data in cancer research, in: von Stechow, L. (Ed.), Methods in Molecular Biology. Springer New York, New York, NY, pp. 133-148. https://doi.org/10.1007/978-1-4939-7493-1_7

Ueno, Y., Nagao, R., Shen, J.-R., Akimoto, S., 2019. Spectral Properties and Excitation Relaxation of Novel Fucoxanthin Chlorophyll a/c-Binding Protein Complexes. J. Phys. Chem. Lett. 10, 5148-5152. https://doi.org/10.1021/acs.jpclett.9b02093 
Ünal, C.M., Steinert, M., 2014. Microbial Peptidyl-Prolyl cis/trans Isomerases (PPIases): Virulence Factors and Potential Alternative Drug Targets. Microbiol. Mol. Biol. Rev. 78, 544-571. https://doi.org/10.1128/mmbr.00015-14

van Oppen, M.J.H., Baker, A.C., Coffroth, M.A., Willis, B.L., 2009. Bleaching resistance and the role of algal endosymbionts, in: Coral Bleaching. Springer, pp. 83-102.

van Oppen, M.J.H., Medina, M., 2020. Coral evolutionary responses to microbial symbioses. Philos. Trans. R. Soc. B 375, 20190591.

Van Oppen, M.J.H., Medina, M., 2020. Coral evolutionary responses to microbial symbioses: Coral-microbe symbiosis. Philos. Trans. R. Soc. B Biol. Sci. 375. https://doi.org/10.1098/rstb.2019.0591rstb20190591

Venn, A.A., Loram, J.E., Douglas, A.E., 2008. Photosynthetic symbioses in animals. J. Exp. Bot. 59, 1069-1080. https://doi.org/10.1093/jxb/erm328

Verma, A., Barua, A., Ruvindy, R., Savela, H., Ajani, P.A., Murray, S.A., 2019. The genetic basis of toxin biosynthesis in dinoflagellates. Microorganisms. https://doi.org/10.3390/microorganisms 708022

Veron, J.E.N., Hoegh-Guldberg, O., Lenton, T.M., Lough, J.M., Obura, D.O., Pearce-Kelly, P., Sheppard, C.R.C., Spalding, M., Stafford-Smith, M.G., Rogers, A.D., 2009. The coral reef crisis: The critical importance of $<350 \mathrm{ppm}$ CO2. Mar. Pollut. Bull. 58, 1428-1436. https://doi.org/10.1016/j.marpolbul.2009.09.009

Vinet, A.F., Fukuda, M., Turco, S.J., Descoteaux, A., 2009. The Leishmania donovani lipophosphoglycan excludes the vesicular proton-ATPase from phagosomes by impairing the recruitment of Synaptotagmin V. PLoS Pathog. 5, e1000628-e1000628. https://doi.org/10.1371/journal.ppat.1000628

Von Holt, C., 1968. Uptake of glycine and release of nucleoside-polyphosphates by zooxanthellae. Comp. Biochem. Physiol 26, 1071-1079. https://doi.org/10.1016/0010-406x(68)90027-3

von Holt, C., von Holt, M., 1968. Transfer of photosynthetic products from zooxanthellae to coelenterate hosts. Comp. Biochem. Physiol. 24, 73-81. https://doi.org/10.1016/0010-406X(68)90959-6

Waddell, S.J., Chung, G.A., Gibson, K.J.C., Everett, M.J., Minnikin, D.E., Besra, G.S., Butcher, P.D., 2005. Inactivation of polyketide synthase and related genes results in the loss of complex lipids in Mycobacterium tuberculosis H37Rv. Lett. Appl. Microbiol. 40, 201-206.

Wakefield, T.S., Farmer, M.A., Kempf, S.C., 2000. Revised description of the fine structure of in situ "Zooxanthellae" genus Symbiodinium. Biol. Bull. 199, 76-84. https://doi.org/10.2307/1542709

Wakefield, T.S., Kempf, S.C., 2001. Development of host- and symbiont-specific monoclonal antibodies and confirmation of the origin of the symbiosome membrane in a cnidarian-dinoflagellate symbiosis. Biol. Bull. 200, 127-143. https://doi.org/10.2307/1543306

Walker, N.S., Fernández, R., Sneed, J.M., Paul, V.J., Giribet, G., Combosch, D.J., 2019. Differential gene expression during substrate probing in larvae of the Caribbean coral Porites astreoides. Mol. Ecol. 28, 4899-4913. https://oi.org/10.1111/mec.15265

Wang, D., Yang, S., Tang, F., Zhu, H., 2012. Symbiosis specificity in the legume - rhizobial mutualism. Cell. Microbiol. 14, 334-342. https://doi.org/10.1111/j.1462-5822.2011.01736.x

Wang, J.T., Douglas, A.E., 1999. Essential amino acid synthesis and nitrogen recycling in an alga-invertebrate symbiosis. Mar. Biol. 135, 219-222. https://doi.org/10.1007/s002270050619

Wang, J.T., Douglas, A.E., 1998. Nitrogen recycling or nitrogen conservation in an alga-invertebrate symbiosis? J. Exp. Biol. 201, 2445-2453.

Wang, L.H., Chen, H.K., Jhu, C.S., Cheng, J.O., Fang, L.S., Chen, C.S., 2015. Different strategies of energy storage in cultured and freshly isolated Symbiodinium sp. J. Phycol. 51, 1127-1136. https://doi.org/10.1111/jpy.12349

Wang, L.H., Lee, H.H., Fang, L.S., Mayfield, A.B., Chen, C.S., 2013. Fatty Acid and Phospholipid Syntheses Are Prerequisites for the Cell Cycle of Symbiodinium and Their Endosymbiosis within Sea Anemones. PLoS One 8, e72486. https://doi.org/10.1371/journal.pone.0072486

Wang, X., Balamurugan, S., Liu, S.-F., Ji, C.-Y., Liu, Y.-H., Yang, W.-D., Jiang, L., Li, H.-Y., 2021. Hydrolysis of organophosphorus by diatom purple acid phosphatase and sequential regulation of cell metabolism. J. Exp. Bot. 72, 2918-2932. https://doi.org/10.1093/jxb/erab026

Ware, J.R., Smith, S. V., Reaka-Kudla, M.L., 1992. Coral reefs: sources or sinks of atmospheric CO2? Coral Reefs 11, 127130. https://doi.org/10.1007/BF00255465 
Warner, M.E., Suggett, D.J., 2016. The Photobiology of Symbiodinium spp.: Linking Physiological Diversity to the Implications of Stress and Resilience BT - The Cnidaria, Past, Present and Future: The world of Medusa and her sisters, in: Goffredo, S., Dubinsky, Z. (Eds.), . Springer International Publishing, Cham, pp. 489-509. https://doi.org/10.1007/978-3-319-31305-4_30

Watanabe, N., Lam, E., 2011. Arabidopsis metacaspase $2 \mathrm{~d}$ is a positive mediator of cell death induced during biotic and abiotic stresses. Plant J. 66, 969-982. https://doi.org/10.1111/j.1365-313X.2011.04554.x

Weber, S.S., Ragaz, C., Hilbi, H., 2009. Pathogen trafficking pathways and host phosphoinositide metabolism. Mol. Microbiol. 71, 1341-1352. https://doi.org/https://doi.org/10.1111/j.1365-2958.2009.06608.x

Weis, V.M., 2008. Cellular mechanisms of Cnidarian bleaching: Stress causes the collapse of symbiosis. J. Exp. Biol. 211, 3059-3066. https://doi.org/10.1242/jeb.009597

Weis, V.M., 1991. The Induction of Carbonic Anhydrase in the Symbiotic Sea Anemone Aiptasia pulchella . Biol. Bull. 180, 496-504. https://doi.org/10.2307/1542351

Weis, V.M., Davy, S.K., Hoegh-Guldberg, O., Rodriguez-Lanetty, M., Pringle, J.R., 2008. Cell biology in model systems as the key to understanding corals. Trends Ecol. Evol. 23, 369-376. https://doi.org/10.1016/j.tree.2008.03.004

Weis, V.M., Reynolds, W.S., Krupp, D.A., 2001. Weis 2001 - Host symbiont Specificity in coral.pdf. Coral reefs 20, 301308.

Weston, A.J., Dunlap, W.C., Shick, J.M., Klueter, A., Iglic, K., Vukelic, A., Starcevic, A., Ward, M., Wells, M.L., Trick, C.G., Long, P.F., 2012. A profile of an endosymbiont-enriched fraction of the coral Stylophora pistillata reveals proteins relevant to microbial-host interactions. Mol. Cell. Proteomics 11, M111-015487. https://doi.org/10.1074/mcp.M111.015487

Wham, D.C., Ning, G., LaJeunesse, T.C., 2017. Symbiodinium glynnii sp. nov., a species of stress-tolerant symbiotic dinoflagellates from pocilloporid and montiporid corals in the Pacific Ocean. Phycologia 56, 396-409. https://doi.org/10.2216/16-86.1

Whitehead, L.F., Douglas, A.E., 2003. Metabolite comparisons and the identity of nutrients translocated from symbiotic algae to an animal host. J. Exp. Biol. 206, 3149-3157. https://doi.org/10.1242/jeb.00539

Wietheger, A., Starzak, D.E., Gould, K.S., Davy, S.K., 2018. Differential ROS Generation in Response to Stress in Symbiodinium spp. Biol. Bull. 234, 11-21. https://doi.org/10.1086/696977

Wilkerson, F.P., Muscatine, L., 1984. Uptake and assimilation of dissolved inorganic nitrogen by a symbiotic sea anemone. Proc. R. Soc. London. Ser. B. Biol. Sci. 221, 71-86. https://doi.org/10.1098/rspb.1984.0023

Wolfowicz, I., Baumgarten, S., Voss, P.A., Hambleton, E.A., Voolstra, C.R., Hatta, M., Guse, A., 2016. Aiptasia sp. larvae as a model to reveal mechanisms of symbiont selection in cnidarians. Sci. Rep. 6, 32366 https://doi.org/10.1038/srep32366

Wong, D., Bach, H., Sun, J., Hmama, Z., Av-Gay, Y., 2011. Mycobacterium tuberculosis protein tyrosine phosphatase (PtpA) excludes host vacuolar-H +-ATPase to inhibit phagosome acidification. Proc. Natl. Acad. Sci. U. S. A. 108, 19371-19376. https://doi.org/10.1073/pnas.1109201108

Wood-Charlson, E.M., Weis, V.M., 2009. The diversity of C-type lectins in the genome of a basal metazoan, Nematostella vectensis. Dev. Comp. Immunol. 33, 881-889. https://doi.org/10.1016/j.dci.2009.01.008

Wood, I.S., Trayhurn, P., 2003. Glucose transporters (GLUT and SGLT): expanded families of sugar transport proteins. Br. J. Nutr. 89, 3-9. https://doi.org/10.1079/bjn2002763

Xiang, T., Hambleton, E.A., Denofrio, J.C., Pringle, J.R., Grossman, A.R., 2013. Isolation of clonal axenic strains of the symbiotic dinoflagellate Symbiodinium and their growth and host specificity. J. Phycol. 49, 447-458. https://doi.org/10.1111/jpy.12055

Xiang, T., Jinkerson, R.E., Clowez, S., Tran, C., Krediet, C.J., Onishi, M., Cleves, P.A., Pringle, J.R., Grossman, A.R., 2018. Glucose-induced trophic shift in an endosymbiont dinoflagellate with physiological and molecular consequences. Plant Physiol. 176, 1793-1807. https://doi.org/10.1104/pp.17.01572

Xiang, T., Lehnert, E., Jinkerson, R.E., Clowez, S., Kim, R.G., DeNofrio, J.C., Pringle, J.R., Grossman, A.R., 2020. Symbiont population control by host-symbiont metabolic interaction in Symbiodiniaceae-cnidarian associations. Nat. Commun. 11, 108. https://doi.org/10.1038/s41467-019-13963-z

Xiang, T., Nelson, W., Rodriguez, J., Tolleter, D., Grossman, A.R., 2015. Symbiodinium transcriptome and global responses of cells to immediate changes in light intensity when grown under autotrophic or mixotrophic conditions. Plant J. 82, 67-80. https://doi.org/10.1111/tpj.12789 
Yamashiro, H., Oku, H., Higa, H., Chinen, I., Sakai, K., 1999. Composition of lipids, fatty acids and sterols in Okinawan corals. Comp. Biochem. Physiol. - B Biochem. Mol. Biol. 122, 397-407. https://doi.org/10.1016/S03050491(99)00014-0

Yang, G., Rose, M.S., Turgeon, B.G., Yoder, O.C., 1996. A polyketide synthase is required for fungal virulence and production of the polyketide T-toxin. Plant Cell 8, 2139-2150. https://doi.org/10.1105/tpc.8.11.2139

Yellowlees, D., Rees, T., Fitt, W., 1994. Effect of Ammonium-supplemented Seawater on Glutamine Synthetase and Glutamate Dehydrogenase Activities in Host Tissue and Zooxanthellae of Pocillopora damicornis and on Ammonium Uptake Rates of the Zooxanthellae.

Yellowlees, D., Rees, T.A. V., Leggat, W., 2008. Metabolic interactions between algal symbionts and invertebrate hosts. Plant, Cell Environ. 31, 679-694. https://doi.org/10.1111/j.1365-3040.2008.01802.x

Yeung, Y., Stanley, E.R., 2010. Rapid detergent removal from peptide samples with ethyl acetate for mass spectrometry analysis. Curr. Protoc. protein Sci. 59, 12-16.

Yoshioka, Y., Yamashita, H., Suzuki, G., Zayasu, Y., Tada, I., Kanda, M., Satoh, N., Shoguchi, E., Shinzato, C., 2021. Whole-Genome Transcriptome Analyses of Native Symbionts Reveal Host Coral Genomic Novelties for Establishing Coral-Algae Symbioses. Genome Biol. Evol. 13. https://doi.org/10.1093/gbe/evaa240

Yuyama, I., Ishikawa, M., Nozawa, M., Yoshida, M., Ikeo, K., 2018. Transcriptomic changes with increasing algal symbiont reveal the detailed process underlying establishment of coral-algal symbiosis. Sci. Rep. 8, 16802. https://doi.org/10.1038/s41598-018-34575-5

Yuyama, I., Ito, Y., Watanabe, T., Hidaka, M., Suzuki, Y., Nishida, M., 2012. Differential gene expression in juvenile polyps of the coral Acropora tenuis exposed to thermal and chemical stresses. J. Exp. Mar. Bio. Ecol. 430-431, 1724. https://doi.org/10.1016/j.jembe.2012.06.020

Yuyama, I., Nakamura, T., Higuchi, T., Hidaka, M., 2016. Different stress tolerances of juveniles of the coral Acropora tenuis associated with clades $\mathrm{C} 1$ and D Symbiodinium. Zool. Stud. 55.

Zamer, W.E., Shick, J.M., 1987. Physiological energetics of the intertidal sea anemone Anthopleura elegantissima - II. Energy balance. Mar. Biol. 93, 481-491. https://doi.org/10.1007/BF00392785

Zhou, H., Guan, Y., Feng, M., Fu, Y., Tachibana, H., Cheng, X., 2020. Evaluation on elongation factor 1 alpha of entamoeba histolytica interaction with the intermediate subunit of the gal/galnac lectin and actin in phagocytosis. Pathogens $9,1-$ 13. https://doi.org/10.3390/pathogens9090702

Ziegler, M., Arif, C., Burt, J.A., Dobretsov, S., Roder, C., LaJeunesse, T.C., Voolstra, C.R., 2017. Biogeography and molecular diversity of coral symbionts in the genus Symbiodinium around the Arabian Peninsula. J. Biogeogr. 44, 674-686. https://doi.org/10.1111/jbi.12913

Ziegler, P., Nikiforov, A., Robinson, B., Bakalinsky, A., O’Donnell, A., Patton-Vogt, J., 2017. Elevated Uptake of Glycerophosphoinositol Through the Git1 Permease Causes Cell Growth Inhibition in Saccharomyces cerevisiae. FASEB J. 31, 781.16-781.16. https://doi.org/https://doi.org/10.1096/fasebj.31.1_supplement.781.16

Zoccola, D., Ganot, P., Bertucci, A., Caminiti-Segonds, N., Techer, N., Voolstra, C.R., Aranda, M., Tambutté, E., Allemand, D., Casey, J.R., Tambutté, S., 2015. Bicarbonate transporters in corals point towards a key step in the evolution of cnidarian calcification. Sci. Rep. 5, 9983. https://doi.org/10.1038/srep09983 


\section{Supplemental Materials}

Due to the large volume of proteomic and molecular data generated in this thesis, all supplementary data have been uploaded to a publicly available repository, as follows:

Supplementary Tables S2.1-S2.7 are available at GitHub (https://github.com/AmirMashini/Breviolum-minutum-Proteome.git).

Supplementary Tables S3.1-S3.5 are available at GitHub (https://github.com/AmirMashini/Symbiont-Colonization-Proteome.git).

Supplementary Tables S4.1-S4.2 and supplementary Figures S4.1-S4.5 are available at GitHub (https://github.com/Amir-Mashini/Aiptasia-Transporters-Quantification-Project.git).

Supplementary Videos SV1-SV4 are available at GitHub (https://github.com/AmirMashini/Aiptasia-Transporters-Quantification-Project/tree/main/3D\%20Reconstruction).

Genotyping results can are available at GitHub (https://github.com/AmirMashini/Genotyping_Results.git) 